\title{
T国
}

\author{
TECHNische UnIVERSITÄT BERLIN \\ FAKULTÄT FÜR ELEKTROTECHNIK UND INFORMATIK \\ LEHRSTUHL FÜR INTELLIGENTE NETZE \\ und Management Verteilter Systeme
}

\section{On the Cost of Concurrency in Transactional Memory}

\author{
vorgelegt von \\ Srivatsan Ravi (M.E.) \\ von der Fakultät IV - Elektrotechnik und Informatik \\ der Technischen Universität Berlin \\ zur Erlangung des akademischen Grades \\ DOKTOR DER INGENIEURWISSENSCHAFTEN (DR.-ING.) \\ genehmigte Dissertation

\section{Promotionsausschuss:} \\ Vorsitzender: Prof. Uwe Nestmann, Ph. D., TU Berlin \\ Gutachterin: Prof. Anja Feldmann, Ph. D., TU Berlin \\ Gutachter: Prof. Petr Kuznetsov, Ph. D., Télécom ParisTech \\ Gutachterin: Prof. Hagit Attiya, Ph. D., The Technion \\ Gutachter: $\quad$ Prof. Rachid Guerraoui, Ph. D., EPFL \\ Gutachter: Prof. Michel Raynal, Ph. D., INRIA, Rennes
}

Tag der wissenschaftlichen Aussprache: 18 June, 2015

Berlin 2015

D 83 



\section{Eidesstattliche Erklärung}

Ich versichere an Eides statt, dass ich diese Dissertation selbständig verfasst und nur die angegebenen Quellen und Hilfsmittel verwendet habe. 



\begin{abstract}
Current general-purpose CPUs are multicores, offering multiple computing units within a single chip. The performance of programs on these architectures, however, does not necessarily increase proportionally with the number of cores. Designing concurrent programs to exploit these multicores emphasizes the need for achieving efficient synchronization among threads of computation. When there are several threads that conflict on the same data, the threads will need to coordinate their actions for ensuring correct program behaviour.
\end{abstract}

Traditional techniques for synchronization are based on locking that provides threads with exclusive access to shared data. Coarse-grained locking typically forces threads to access large amounts of data sequentially and, thus, does not fully exploit hardware concurrency. Program-specific fine-grained locking or non-blocking (i.e., not using locks) synchronization, on the other hand, is a dark art to most programmers and trusted to the wisdom of a few computing experts. Thus, it is appealing to seek a middle ground between these two extremes: a synchronization mechanism that relieves the programmer of the overhead of reasoning about data conflicts that may arise from concurrent operations without severely limiting the program's performance. The Transactional Memory (TM) abstraction is proposed as such a mechanism: it intends to combine an easy-to-use programming interface with an efficient utilization of the concurrent-computing abilities provided by multicore architectures. TM allows the programmer to speculatively execute sequences of shared-memory operations as atomic transactions with all-or-nothing semantics: the transaction can either commit, in which case it appears as executed sequentially, or abort, in which case its update operations do not take effect. Thus, the programmer can design software having only sequential semantics in mind and let TM take care, at run-time, of resolving the conflicts in concurrent executions.

Intuitively, we want TMs to allow for as much concurrency as possible: in the absence of severe data conflicts, transactions should be able to progress in parallel. But what are the inherent costs associated with providing high degrees of concurrency in TMs? This is the central question of the thesis.

To address this question, we first focus on the consistency criteria that must be satisfied by a TM implementation. We precisely characterize what it means for a TM implementation to be safe, i.e., to ensure that the view of every transaction could have been observed in some sequential execution. We then present several lower and upper bounds on the complexity of three classes of safe TMs: blocking TMs that allow transactions to delay or abort due to overlapping transactions, non-blocking TMs which adapt to step contention by ensuring that a transaction not encountering steps of overlapping transactions must commit, and partially non-blocking TMs that provide strong non-blocking guarantees (wait-freedom) to only a subset of transactions. We then propose a model for hybrid TM implementations that complement hardware transactions with software transactions. We prove that there is an inherent trade-off on the degree of concurrency allowed between hardware and software transactions and the costs introduced on the hardware. Finally, we show that optimistic synchronization techniques based on speculative executions are, in a precise sense, better equipped to exploit concurrency than inherently pessimistic techniques based on locking. 


\section{Zusammenfassung}

Aktuelle Allzweck-CPUs haben mehrere Rechenkerne innerhalb eines einzelnen Chipsatzes. Allerdings erhöht sich die Leistung der Programme auf diesen Architekturen nicht notwendigerweise proportional in der Anzahl der Kerne. Das Entwerfen nebenläufiger Programme um diese Multicores zu nutzen, erfordert die Überwindung einiger nicht-trivialer Herausforderungen; die wichtigste ist, eine effiziente Synchronisierung der Threads der Berechnung herzustellen. Greifen mehrere Threads gleichzeitig auf dieselben Daten zu, müssen diese ihre Aktionen koordinieren, um ein korrektes Programmverhalten zu gewährleisten.

Die traditionelle Methode zur Synchronisierung ist "Locking", welches jeweils nur einem einzelnen Thread Zugriff auf gemeinsam genutzten Daten gewährt. Bei grobkörnigem "Lockingërfolgt der Zugang zu einer großen Menge von Daten meist seriell, sodass die Hardware-Parallelität nich in vollem Unfang ausgenutzt wird. Auf der anderen Seite stellt programmspezifisches feinkörniges Locking, oder auch nicht-blockierende (d.h. keine Locks benutzende) Synchronisierung, eine dunkle Kunst für die meisten Programmierer dar, welche auf die Weisheit weniger Computerexperten vertraut. So ist es angebracht, einen Mittelweg zwischen diesen beiden Extremen zu suchen: einen Synchronisierungsmechanismus, der den Programmierer bezüglich der Datenkonflikte, die aus gleichzeitigen Operationen entstehen, entlastet, ohne jedoch die Leistung des Programms zu stark zu beeinträchtigen. Die Transactional Memory (TM) Abstraktion wird als solcher Mechanismus vorgeschlagen: ihr Ziel ist es, eine einfach zu bedienende Programmierschnittstelle mit einer effizienten Nutzung der gleichzeitigen Computing-Fähigkeiten von Multicore-Architekturen zu kombinieren. TM erlaubt es dem Programmierer, Sequenzen von Operationen auf dem gemeinsamen Speicher als atomare Transaktionen mit Alles-oder-Nichts Semantik zu erklären: Die Transaktion wird entweder übergeben, und somit sequentiell ausgeführt, oder abgebrochen, sodass ihre Operationen nicht durchgeführt werden. Dies ermöglicht dem Programmierer, Software mit nur sequentieller Semantik zu konzipieren, und die aus gleichzeitger Ausführung entstehenden Konflikte TM zu überlassen.

Intuitiv sollen die TMs so viel Nebenläufigkeit wie möglich berücksichtigen: Falls keine Datenkonflikte vorhanden sind, sollen alle Transaktionen parallel ausgeführt werden. Gibt es in TMs Kosten, die durch diesen hohen Grad an Nebenläufigkeit entstehen? Das ist die zentrale Frage dieser Arbeit.

Um diese Frage zu beantworten, konzentrieren wir uns zunächst auf das Kriterium der Konsistenz, welche von der TM-Implementierung erfüllt werden muss. Wir charakterisieren auf präzise Art, was es für eine TM-Implementierung heißt, sicher zu sein, d.h. zu gewährleisten, dass die Sicht einer jeden Transaktion auch von einer sequentiellen Transaktion hätte beobachtet werden können. Danach präsentieren wir mehrere untere und obere Schranken für die Komplexität dreier Klassen von sicheren TMs: blockierende TMs, die Blockierungen oder Abbrüche der Transaktionen erlauben, sollten diese sich überlappen, nicht-blockierende TMs die einen schrittweisen Zugriffskonflikt berücksichtigen, d.h. Transaktionen, die keinen Zugriff überlappender anderer Transaktionen beobachten, müssen übergeben, und partiell nichtblockierende TMs, die nur für eine Teilmenge von Transaktionen nicht-blockierend sind. Wir schlagen daraufhin ein Modell für hybride TM-Implementierungen vor, welches die Hardware Transaktionen mit Software Transaktionen ergänzt. Wir beweisen, dass es eine inherente Trade-Off zwischen Grad der erlaubten Nebenläufigkeit zwischen Hard- und Software Transaktionen und den Kosten der Hardware gibt. Schlussendlich beweisen wir, dass optimistische, auf spekulativen Ausführungen basierende, Synchronisierungstechniken, in einem präzisen Sinne, besser geeignet sind um Nebenläufigkeit auszunutzen als pessimistische Techniken, die auf "Locking"basieren. 


\section{Contents}

\begin{tabular}{lll}
\hline Introduction & 11 \\
\hline
\end{tabular}

1.1 Concurrency and synchronization . . . . . . . . . . . . . . . . . . . . 11

1.1 .1 Concurrent computing overview . . . . . . . . . . . . . . . . . . . . . . . . . . . . . . . . . . . 11

1.1 .2 Synchronization using locks . . . . . . . . . . . . . . . . . . . . . . 12

$1.1 .3 \quad$ Non-blocking synchronization . . . . . . . . . . . . . . . . . . . . . . . . . . . . . . . . 13

1.2 Transactional Memory $(\mathrm{TM}) \quad \ldots \ldots \ldots \ldots \ldots$. . . . . . . . . . . . . . . 14

1.3 Summary of the thesis . . . . . . . . . . . . . . . . . . . . . . . . 15

1.3.1 Safety for transactional memory . . . . . . . . . . . . . . . . . . . . . . . . . . . . . . . . . 15

1.3 .2 Complexity of transactional memory . . . . . . . . . . . . . . . . . . . . . . . . . . . . . . . . .

1.3.3 Hybrid transactional memory . . . . . . . . . . . . . . . . . . . . . . 17

1.3.4 Optimism for boosting concurrency . . . . . . . . . . . . . . . . . . . . . . 18

1.4 Roadmap of the thesis . . . . . . . . . . . . . . . . . . . . . . 19

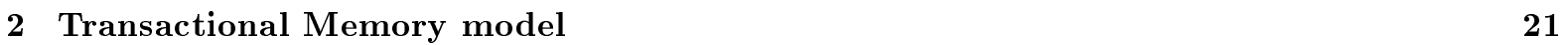

2.1 TM interface and TM implementations . . . . . . . . . . . . . . . . . . . . . . 21

2.2 TM-correctness . . . . . . . . . . . . . . . . . . . . . . . . . . . . . . 24

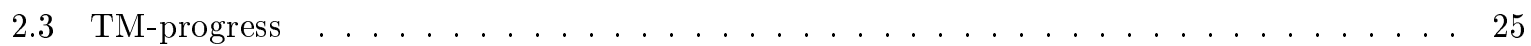

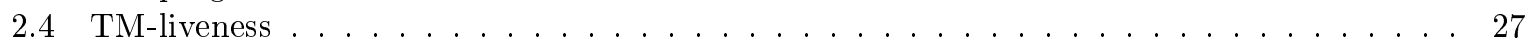

2.5 Invisible reads $\ldots \ldots \ldots \ldots \ldots \ldots \ldots \ldots \ldots \ldots \ldots \ldots$

2.6 Disjoint-access parallelism (DAP) . . . . . . . . . . . . . . . . . . . . . 29

2.7 TM complexity metrics $\ldots \ldots \ldots \ldots \ldots \ldots \ldots \ldots \ldots \ldots$

\begin{tabular}{|lll}
\hline & Safety for transactional memory & 33
\end{tabular}

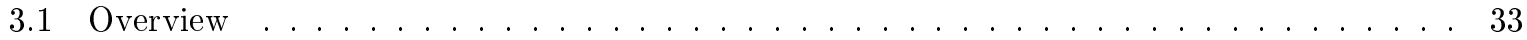

3.2 Safety properties . . . . . . . . . . . . . . . . . . . . . 34

$3.3 \quad$ Opacity and deferred-update(DU) semantics . . . . . . . . . . . . . . . . . . . . 35

3.4 On the safety of du-opacity . . . . . . . . . . . . . . . . . . . . 36

$3.4 .1 \quad$ Du-opacity is prefix-closed . . . . . . . . . . . . . . . . . . . 36

3.4 .2 The limit of du-opaque histories . . . . . . . . . . . . . . . . . . . . . 37

3.4 .3 Du-opacity is limit-closed for complete histories . . . . . . . . . . . . . . . . . . . . 38

3.4.4 Du-opacity vs. opacity . . . . . . . . . . . . . . . . . . . 41

3.5 Strict serializability with DU semantics . . . . . . . . . . . . . . . . . . . . . 43

3.6 Du-opacity vs. other deferred-update criteria . . . . . . . . . . . . . . . . . . . . . 43

3.6.1 Virtual-world consistency . . . . . . . . . . . . . . . . . . . . . . . . 44

3.6.2 Transactional memory specification (TMS) . . . . . . . . . . . . . . . . . . . . 45

3.7 Related work and Discussion . . . . . . . . . . . . . . . . . . . . 48

4 Complexity bounds for blocking TMs $\quad 49$

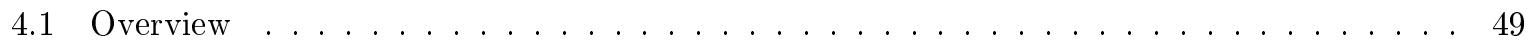

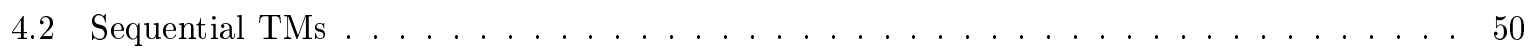

4.2.1 A quadratic lower bound on step complexity . . . . . . . . . . . . . . 51

4.2.2 Expensive synchronization in Transactional memory cannot be eliminated . . . . . 53

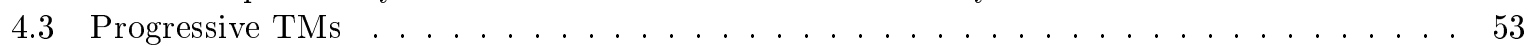

4.3.1 A linear lower bound on the amount of protected data . . . . . . . . . . . . . . 54

4.3.2 A constant stall and constant expensive synchronization strict DAP opaque TM . 55 
4.4 Strongly progressive TMs . . . . . . . . . . . . . . . . . . . . 61

$4.4 .1 \quad \mathrm{~A} \Omega(n \log n)$ lower bound on remote memory references . . . . . . . . . . . . 61

$4.4 .2 \quad$ A constant expensive synchronization opaque TM . . . . . . . . . . . . . . 65

4.5 On the cost of permissive opaque $\mathrm{TMS} \ldots \ldots \ldots \ldots$. . . . . . . . . . . . 68

4.6 Related work and Discussion . . . . . . . . . . . . . . . . . . . . 70

5 Complexity bounds for non-blocking TMs

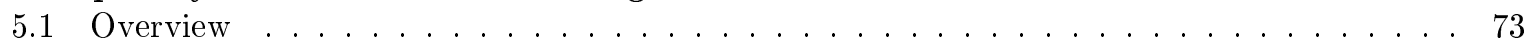

5.2 Impossibility of weak DAP and invisible reads . . . . . . . . . . . . . . . . . . 73

5.3 A linear lower bound on memory stall complexity . . . . . . . . . . . . . . . . 76

5.4 A linear lower bound on expensive synchronization for RW DAP . . . . . . . . . . . . . . 78

5.5 Algorithms for obstruction-free TMs . . . . . . . . . . . . . . . . . . 81

5.5 .1 An opaque RW DAP TM implementation . . . . . . . . . . . . . . . . . . . . . . . 82

5.5 .2 An opaque weak DAP TM implementation . . . . . . . . . . . . . . . . . . 86

5.6 Why Transactional memory should not be obstruction-free . . . . . . . . . . . . . . 87

5.7 Related work and Discussion $\ldots \ldots \ldots \ldots \ldots$. . . . . . . . . . . . 88

6 Lower bounds for partially non-blocking TMs 91

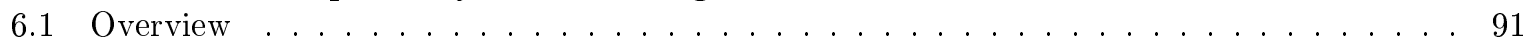

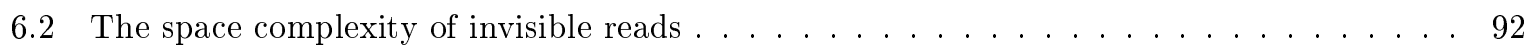

6.3 Impossibility of strict DAP $\ldots \ldots \ldots \ldots \ldots \ldots$

6.4 A linear lower bound on expensive synchronization for weak DAP . . . . . . . . . . . . . . 95

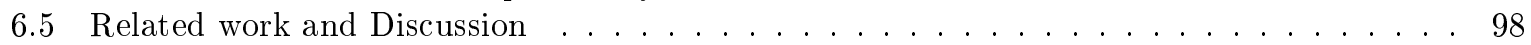

\begin{tabular}{|ll}
\hline Hybrid transactional memory (HyTM) & 99
\end{tabular}

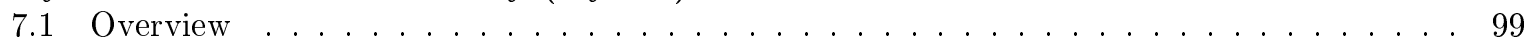

7.2 Modelling HyTM . . . . . . . . . . . . . . . . . . . . . . . 101

7.2.1 Direct and cached accesses . . . . . . . . . . . . . . . . . . . . . 101

7.2 .2 Slow-path and fast-path transactions . . . . . . . . . . . . . . . . . . . 102

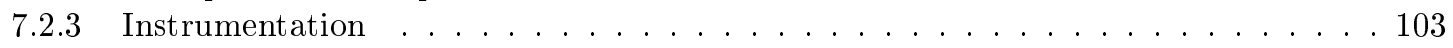

7.2 .4 Impossibility of uninstrumented HyTMs . . . . . . . . . . . . . . . . . . . . . . . . 104

7.3 A linear lower bound on instrumentation for progressive HyTMs . . . . . . . . . . . . . 106

7.4 Instrumentation-optimal progressive HyTM . . . . . . . . . . . . . . . . . . . . 111

7.5 Providing partial concurrency at low cost . . . . . . . . . . . . . . . . . . 116

7.6 Related work and Discussion . . . . . . . . . . . . . . . . . . . . . 117

\begin{tabular}{|lr|}
\hline Optimism for boosting concurrency & 119
\end{tabular}

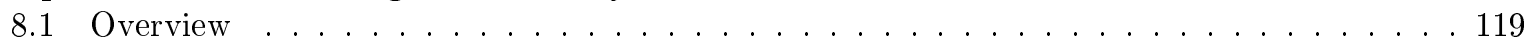

8.2 Concurrent implementations . . . . . . . . . . . . . . . . . . . . . . 121

8.3 Locally serializable linearizability . . . . . . . . . . . . . . . . . . . . . . . . . . . 123

8.4 Pessimistic vs. optimistic synchronization . . . . . . . . . . . . . . . . . . . . . . 125

8.4 .1 Concurrency analysis . . . . . . . . . . . . . . . . . . 126

8.4 .2 Concurrency optimality . . . . . . . . . . . . . . . . . . . 127

8.5 Related work and Discussion . . . . . . . . . . . . . . . . . . . . . 132

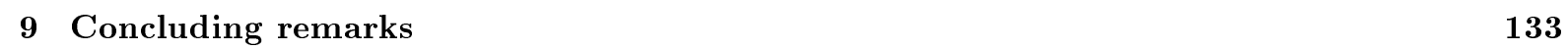

\begin{tabular}{|rr}
\hline List of Figures & 137 \\
\hline
\end{tabular}

\begin{tabular}{lr}
\hline List of Tables & 139
\end{tabular}

\begin{tabular}{|rr}
\hline 10 Bibliography & 141
\end{tabular} 


\section{Acknowledgements}

In his wonderfully sarcastic critique of the scientific community in His Master's Voice, the great Polish writer Stanisław Lem refers to a specialist as a barbarian whose ignorance is not well-rounded. Writing a Ph.D. thesis is essentially an attempt at becoming a specialist on some topic; whether this thesis on Transactional Memory makes me one is a questionable claim, but I am culturedly not totally ignorant, I think. The thesis itself was a long time in the making and would not have been possible without the wonderful support and gratitudes I have received these past four years.

My advisors Anja Feldmann and Petr Kuznetsov guided me throughout my Ph. D. term.

A fair amount of whatever good I have learnt these past few years, both scientifically and metascientifically, I owe it to Petr. He taught me, by example, what it takes to achieve nontrivial scientific results. He spent several hours schooling me when I had misunderstood some topic and as such suffered the worst of my writing, especially in the first couple of years. He was hard on me when I did badly, but always happy for me when I did well. Apart from being a deep thinker and a brilliant researcher, his scientific integrity and mental discipline have indelibly made me a better human being and student of science. At a personal level, I wish to thank him and his family for undeserved kindness shown to me over the years.

I would like to thank Anja for the extraordinary amount of freedom she gave me to pursue my own research and the trust she placed in me, as she does in all her students.

I am especially grateful to Robbert Van Renesse and Bryan Ford, who gave me a taste for independent research and in many ways, helped shape the course of my graduate career.

I am of course extremely grateful to all my co-authors who allowed me to include content, written in conjunction with them, in the thesis. So special thanks to Dan Alistarh, Hagit Attiya, Vincent Gramoli, Sandeep Hans, Justin Kopinsky, Petr Kuznetsov and Nir Shavit.

The results in Chapter 3 were initiated during a memorable visit to the Technion, Haifa in the Spring of '12. I am thankful to have been hosted by Hagit Attiya and to have had the chance to work with her and Sandeep Hans. I am also very grateful to David Sainz for taking time off and introducing me to some beautiful parts of Israel.

Chapter 7 essentially stemmed from a visit to MIT in the summer of '13. I am very grateful to Dan Alistarh and Nir Shavit for hosting me. Special thanks to Justin Kopinsky, who helped keep our discussions alive during our lengthy dry spells when we were seemingly spending all our time thinking about the problem, but without producing any tangible results.

Chapter 8 represents the most excruciatingly painful part of the thesis purely in terms of the number of iterations the paper based on this chapter went through. Yet, it was a procedure from which I learnt a lot and I am very thankful to Vincent Gramoli, who initiated the topic during my visit to EPFL in Spring ' 11 .

In general, I have benefitted immensely from just talking to researchers in distributed computing during conferences, workshops and research visits. These include Yehuda Afek, Panagiota Fatourou, Rachid Guerraoui, Maurice Herlihy, Victor Luchangco, Adam Morrison and Michel Raynal. Also, great thanks to the anonymous reviewers of my paper submissions whose critiques and comments helped improve the contents of the thesis.

Back here in Berlin, so many of my INET colleagues have shaped my thought processes and enriched my experience in grad school. Thanks to Stefan Schmid, whose ability to execute several tasks concurrently with minimal synchronization overhead, never ceases to amaze anyone in this group. I am also very grateful to Anja, Petr and Stefan for allowing me to be a Teaching Assistant in their respective courses. Apart from being great friends, Felix Poloczek and Matthias Rost have been wonderful office mates and indulged my random discussions about life and research. Arne Ludwig and Carlo Fürst have been great friends; Arne, thanks for all the football discussions and Carlo, for exposing me to some social life. Thanks to Dan Levin, who has been a great friend and always been there to motivate and give me a fillip whenever I needed it. Ingmar Poese has been a wonderful friend as well as a constant companion to the 
movies. Franziska Lichtblau, Enric Pujol, Philipp Richter and others have been willing companions in ordering several late night dinners at the lab. Thomas Krenc and Philipp Schmidt were great co-TA's. Great thanks to all the other current and past members of INET: our system admin Rainer May, Marco Canini, Damien Foucard, Juhoon Kim, Gregor Schaffrath, Julius Schulz-Zander, Georgios Smaragdakis, Florian Streibelt, Lalith Suresh, Steve Uhlig and all the other members I have missed. Special thanks to our group secretaries, Birgit Hohmeier-Touré and Nadine Pissors, without whom there would be absolute chaos.

I would like to thank my flat mates of the last two years: Ingmar, Jennifer, Jose, Lily and Mathilda. Lastly, thanks to my family and friends outside of the academic sphere for tolerating me all these years. 


\section{1 \\ Introduction}

While the performance of programs would increase proportionally with the performance of a singlecore $\mathrm{CPU}$, the performance of programs on multicore CPU architectures, however, does not necessarily increase proportionally with the number of cores. In order to exploit these multicores, the amount of concurrency provided by programs will need to increase as well. Designing concurrent programs that exploit the hardware concurrency provided by modern multicore CPU architectures requires achieving efficient synchronization among threads of computation. However, due to the asynchrony resulting from the CPU's context switching and scheduling policies, it is hard to specify reasonable bounds on relative thread speeds. This makes the design of efficient and correct concurrent programs a difficult task. The Transactional Memory (TM) abstraction [78, 114] is a synchronization mechanism proposed as a solution to this problem: it combines an easy-to-use programming interface with an efficient utilization of the concurrent-computing abilities provided by multicore architectures. This chapter introduces the TM abstraction and presents an overview of the thesis.

\subsection{Concurrency and synchronization}

In this section, we introduce the challenges of concurrent computing and overview the drawbacks associated with traditional synchronization techniques.

\subsubsection{Concurrent computing overview}

Shared memory model. A process represents a thread of computation that is provided with its own private memory which cannot be accessed by other processes. However, these independent processes will have to synchronize their actions in an asynchronous environment in order to implement a user application, which they do by communicating via the CPU's shared memory.

In the shared memory model of computation, processes communicate by reading and writing to a fragment of the shared memory, referred to as a base object, in a single atomic (i.e., indivisible) instruction. Modern CPU architectures additionally allow processes to invoke certain powerful atomic read-modify-write (rmw) instructions [73, which allow processes to write to a base object subject to the check of an invariant. For example, the compare-and-swap instruction is a rmw instruction that is supported by most modern architectures: it takes as input $\langle$ old, new $\rangle$ and atomically updates the value of a base object to new and returns true iff its value prior to applying the instruction is equal to old; otherwise it returns false. 
Concurrent implementations. A concurrent implementation provides each process with an algorithm to apply CPU instructions on the shared base objects for the operations of the user application. For example, consider the problem of implementing a concurrent list-based set [79]. the set abstraction implemented as a sorted linked list supporting operations $\operatorname{insert}(v)$, remove $(v)$ and $\operatorname{contains}(v) ; v \in \mathbb{Z}$. The set abstraction stores a set of integer values, initially empty. The update operations, $\operatorname{insert}(v)$ and remove $(v)$, return a boolean response, true if and only if $v$ is absent (for insert $(v)$ ) or present (for remove $(v))$ in the list. After insert $(v)$ is complete, $v$ is present in the list, and after remove $(v)$ is complete, $v$ is absent in the list. The contains $(v)$ returns a boolean, true if and only if $v$ is present in the list. A concurrent implementation of the list-based set is simply an emulation of the set abstraction that is realized by processes applying the available $\mathrm{CPU}$ instructions on the underlying base objects.

Safety and liveness. What does it mean for a concurrent implementation to be correct? Firstly, the implementation must satisfy a safety property: there are no bad reachable states in any execution of the implementation. Intuitively, we characterize safety for a concurrent implementation of a data abstraction by verifying if the responses returned in the concurrent execution may have been observed in a sequential execution of the same. For example, the safety property for a concurrent list-based set implementation stipulates that the response of the set operations in a concurrent execution is consistent with some sequential execution of the list-based set. However, a concurrent set implementation that does not return any response trivially ensures safety; thus, the implementation must satisfy some liveness property specifying the conditions under which the operations must return a response. For example, one liveness property we may wish to impose on the concurrent list-based set is wait-freedom: every process completes the operations it invokes irrespective of the behaviour of other processes.

As another example, consider the mutual exclusion problem [40] which involves sharing some critical data resource among processes. The safety property for mutual exclusion stipulates that at most one process has access to the resource in any execution, in which case, we say that the process is inside the critical section. However, one may notice that an implementation which ensures that no process ever enters the critical section is trivially safe, but not very useful. Thus, the mutual exclusion implementation must satisfy some liveness property specifying the conditions under which the processes must eventually enter the critical section. For example, we expect that the implementation is deadlock-free: if every process is given the chance to execute its algorithm, some process will enter the critical section. In contrast to safety, a liveness property can be violated only in an infinite execution, e.g., by no process ever entering the critical section.

In shared memory computing, we are concerned with deriving concurrent implementations with strong safety and liveness properties, thus emphasizing the need for efficient synchronization among processes.

\subsubsection{Synchronization using locks}

A lock is a concurrency abstraction that implements mutual exclusion and is the traditional solution for achieving synchronization among processes. Processes acquire a lock prior to executing code inside the critical section and release the lock afterwards, thereby allowing other processes to modify the data accessed by the code within the critical section. In essence, after acquiring the lock, the code within the critical section can be executed atomically. However, lock-based implementations suffer from some fundamental drawbacks.

Ease of designing lock-based programs. Ideally, to reduce the programmer's burden, we would like to take any sequential implementation and transform it to an efficient concurrent one with minimal effort. Consider a simple locking protocol that works for most applications: coarse-grained locking which typically serializes access to a large amount of data. Although trivial for the programmer to implement, it does not exploit hardware concurrency. In contrast, fine-grained locking may exploit concurrency better, but requires the programmer to have a good understanding of the data-flow relationships in the application and precisely specify which locks provide exclusive access to which data.

For example, consider the problem of implementing a concurrent list-based set. The sequential implementation of the list-based set uses a sorted linked list data structure in which each data item (except 
the tail of the list) maintains a next field to provide a pointer to the successor. Every operation (insert, remove and contains) invoked with a parameter $v \in \mathbb{Z}$ traverses the list starting from the head up to the data item storing value $v^{\prime} \geq v$. If $v^{\prime}=v$, then contains returns true, remove $(v)$ unlinks the corresponding element and returns true and $\operatorname{insert}(v)$ returns false. Otherwise, contains $(v)$ and remove $(v)$ return false, while insert $(v)$ adds a new data item with value $v$ to the list and returns true.

Given such a sequential implementation, we may derive a coarse-grained implementation of the list-based set by having processes acquire a lock on the head of the list, thus, forcing one operation to complete before the next starts. Alternatively, a fine-grained protocol may involve acquiring locks hand-overhand [28]: a process holds the lock on at most two adjacent data items of the list. Yet, while such a protocol produces a correct set implementation [25], it is not a universal strategy that applies to other popular data abstractions like queues and stacks.

Composing lock-based programs. It is hard to compose smaller atomic operations based on locks to produce a larger atomic operation without affecting safety [79, 114. Consider the fifo queue abstraction supporting the enqueue $(v) ; v \in \mathbb{Z}$ and dequeue operations. Suppose that we wish to solve the problem of atomically dequeuing from a queue $Q_{1}$ and enqueuing the item returned, say $v$, to a queue $Q_{2}$. While the individual actions of dequeuing from $Q_{1}$ and enqueuing $v$ to $Q_{2}$ may be atomic, we wish to ensure that the combined action is atomic: no process must observe the absence of $v$ or that it is present in both $Q_{1}$ and $Q_{2}$. A possible solution to this specific problem is to force a process attempting atomic modification of $Q_{1}$ and $Q_{2}$ to acquire a lock. Firstly, this requires prior knowledge of the identities of the two queue instances. Secondly, this solution does not exploit hardware concurrency since the lock itself becomes a concurrency bottleneck. Moreover, imagine that processes $p_{1}$ and $p_{2}$ need to acquire two locks $L_{1}$ and $L_{2}$ in order to atomically modify a set of queue instances. Without imposing a pre-agreed upon order on such lock acquisitions, there is the possibility of introducing deadlocks where processes wait infinitely long without completing their operations. For example, imagine the following concurrency scenario: process $p_{1}$ (and resp., $p_{2}$ ) holds the lock $L_{1}$ (and resp., $L_{2}$ ) and attempts to acquire the lock $L_{2}$ (and resp., $L_{1}$ ). Thus, process $p_{1}$ (and resp., $p_{2}$ ) waits infinitely long for $p_{2}$ (and resp., $p_{1}$ ) to complete its operation.

\subsubsection{Non-blocking synchronization}

It is impossible to derive lock-based implementations that provide non-blocking liveness guarantees, i.e., some process completes its operations irrespective of the behaviour of other processes. In fact, even the weak non-blocking liveness property of obstruction-freedom [15] cannot be satisfied by lock-based implementations: a process must complete its operation if it eventually runs solo without interleaving events of other processes.

Concurrent implementations providing non-blocking liveness properties are appealing in practice since they overcome problems like deadlocks and priority inversions [29] inherent to lock-based implementations. Thus, non-blocking (without using locks) solutions using conditional rmw instructions like compare-and-swap have been proposed as an alternative to lock-based implementations. However, as with fine-grained locking, implementing correct non-blocking algorithms can be hard and requires handcrafted problem-specific strategies. For example, the state-of-the-art list-based set implementation by Harris-Michael [68, 79, 102] is non-blocking: the insert and remove operations, as they traverse the list, help concurrent operations to physically remove data items (using compare-and-swap) that are logically deleted, i.e., "marked for deletion". But one cannot employ an identical algorithmic technique for implementing a non-blocking queue [103, whose semantics is orthogonal to that of the set abstraction. Moreover, addressing the compositionality issue, as with lock-based solutions, requires ad-hoc strategies that are not easy to realize 79 . 

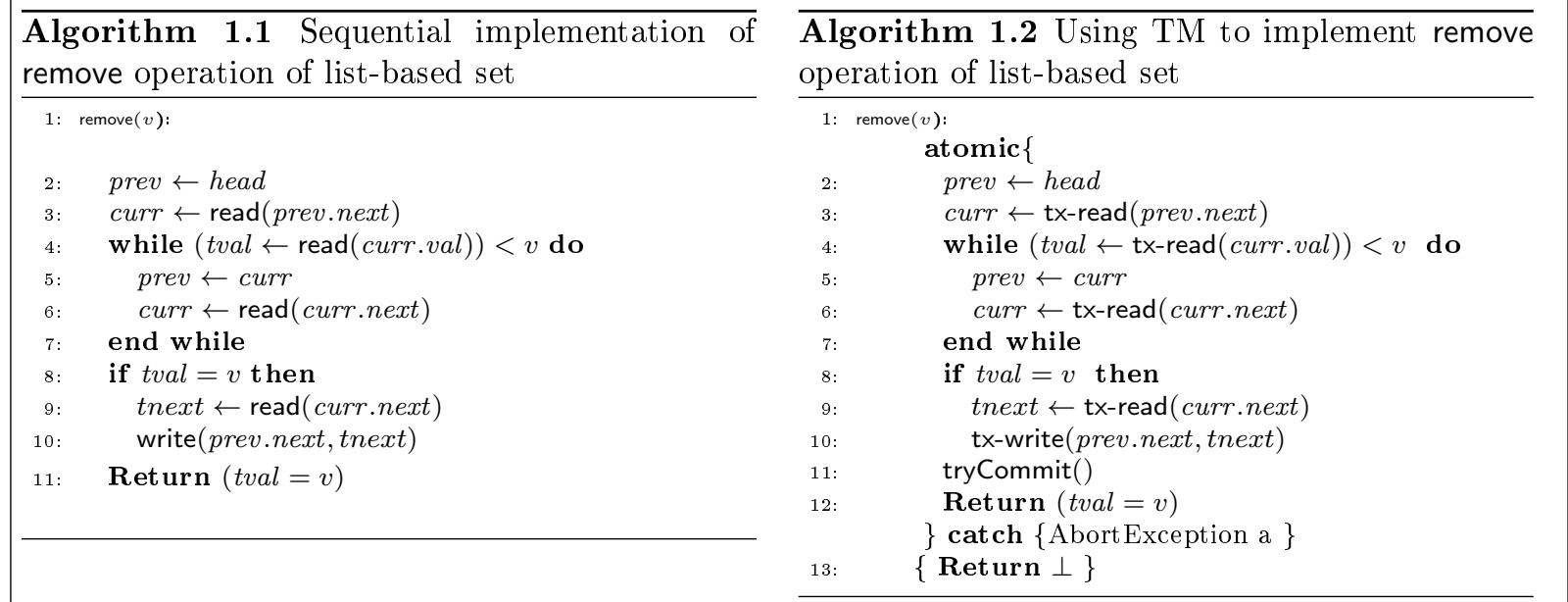

Figure 1.1: Transforming a sequential implementation of the list-based set to a TM-based concurrent one

\subsection{Transactional Memory (TM)}

Transactional Memory (TM) [78, 114] addresses the challenge of resolving conflicts (concurrent reading and writing to the same data) in an efficient and safe manner by offering a simple interface in which sequences of shared memory operations on data items can be declared as optimistic transactions. The underlying idea of TM, inspired by databases [56], is to treat each transaction as atomic: a transaction may either commit, in which case it appears as executed sequentially, or abort, in which case none of its update operations take effect. Thus, it enables the programmer to design software applications having only sequential semantics in mind and let TM take care of dynamically handling the conflicts resulting from concurrent executions at run-time.

A TM implementation provides processes with algorithms for implementing transactional operations such as read, write, tryCommit and tryAbort on data items using base objects. TM implementations typically ensure that all committed transactions appear to execute sequentially in some total order respecting the timing of non-overlapping transactions. Moreover, unlike database transactions, intermediate states witnessed by the read operations of an incomplete transaction may affect the user application. Thus, to ensure that the TM implementation does not export any pathological executions, it is additionally expected that every transaction (including aborted and incomplete ones) must return responses that is consistent with some sequential execution of the TM implementation.

In general, given a sequential implementation of a data abstraction, a corresponding TM-based concurrent one encapsulates the sequential (high-level) operations within a transaction. Then, the TM-based concurrent implementation of the data abstraction replaces each read and write of a data item with the transactional read and write implementations, respectively. If the transaction commits, then the result of the high-level operation is returned to the application. Otherwise, one of the transactional operations may be aborted, in which case, the write operations performed by the transaction do not take effect and the high-level operation is typically re-started with a new transaction.

To illustrate this, we refer to the sequential implementation of the remove operation of list-based set depicted in Algorithm 1.1 of Figure 1.1. In a TM-based concurrent implementation of the list-based set (Algorithm 1.2), each read (and resp. write) operation performed by remove $(v)$ on a data item $X$ of the list is replaced with tx-read $(X)$ (and resp., tx-write $(X, \arg )$ ). tx-read $(X)$ returns the value of the data item $X$ or aborts the transaction while tx-write $(X, \arg )$ writes the value $\arg$ to $X$ or aborts the transaction. Finally, the process attempts to commit the transaction by invoking the tryCommit operation. If the tryCommit is successful, the response of remove $(v)$ is returned; otherwise a failed response $($ denoted $\perp)$ is returned, in which case, the write operations performed by the transaction are "rolled back". 
Intuitively, it is easy to understand how TM simplifies concurrent programming. Deriving a TM-based concurrent implementation of the list-based set simply requires encapsulating the operations to be executed atomically within a transaction using an atomic delimiter 1 . The underlying TM implementation endeavours to dynamically execute the transactions by resolving the conflicts that might arise from processes reading and writing to the same data item at run-time. Intuitively, since the TM implementation enforces a strong safety property, the resulting list-based implementation is also safe: the responses of its operations are consistent with some sequential execution of the list-based set.

One may view TM as a universal construction [12, 27, 44, 48, 73, that accepts as input the operations of a sequential implementation and strives to execute them concurrently. Specifically, TM is designed to work in a dynamic environment where neither the sequence of operations nor the data items accessed by a transaction are known a priori. Thus, the response of a read operation performed by a transaction is returned immediately to the application, and the application determines the next data item that must accessed by the transaction.

TM-based implementations overcome the drawbacks of traditional synchronization techniques based on locks and compare-and-swap. Firstly, the TM interface places minimal overhead on the programmer: using TM only requires encapsulating the sequential operations within transactions and handling an exception should the transaction be aborted. Secondly, the ability to execute multiple operations atomically allows TM-based implementations to seamlessly compose smaller atomic operations to produce larger ones. For example, suppose that we wish to atomically dequeue from a queue $Q_{1}$ and enqueue $(v)$ in queue $Q_{2}$, where $v$ is the value returned by $Q_{1}$.dequeue. Solving this problem using TM simply requires encapsulating the sequential implementation of $Q_{1}$.dequeue, followed by $Q_{2}$.enqueue $(v)$ within a transaction.

Note that a TM implementation may internally employ locks or conditional rmw instructions like compare-and-swap. However, TM raises the level of abstraction by exposing an easy-to-use compositional transactional interface for the user application that is oblivious to the specifics of the implementation and the semantics of the user application.

\subsection{Summary of the thesis}

TM allows the programmer to speculatively execute sequences of shared-memory operations as atomic transactions: if the transaction commits, the operations appear as executed sequentially, or if the transaction aborts, the update operations do not take effect. The combination of speculation and the simple programming interface provided by TM seemingly overcomes the problems associated with traditional synchronization techniques based on locks and compare-and-swap. But are there some fundamental drawbacks associated with the TM abstraction? Does providing high degrees of concurrency in TMs come with inherent costs? This is the central question of the thesis. In the rest of this introductory chapter, we provide a summary of the results in the thesis that give some answers to this question.

\subsubsection{Safety for transactional memory}

We first need to define the consistency criteria that must be satisfied by a TM implementation. We formalize the semantics of a safe TM: every transaction, including aborted and incomplete ones, must observe a view that is consistent with some sequential execution. This is important, since if the intermediate view is not consistent with any sequential execution, the application may experience a fatal irrevocable error or enter an infinite loop. Additionally, the response of a transaction's read should not depend on an ongoing transaction that has not started committing yet. This restriction, referred to as deferred-update semantics appears desirable, since the ongoing transaction may still abort, thus rendering the read inconsistent. We define the notion of deferred-update semantics formally and apply it to several TM consistency criteria proposed in literature. We then verify if the resulting TM consistency

${ }^{1}$ Different compilers may use different names for the delimiter; in $G C C$, it is transaction_atomic [2] 
criterion is a safety property [10, 97, 105, in the formal sense, i.e., the set of histories (interleavings of invocations and responses of transactional operations) is prefix-closed and limit-closed.

We first consider the popular consistency criterion of opacity 62. Opacity requires the states observed by all transactions, included uncommitted ones, to be consistent with a global serialization, i.e., a serial execution constituted by committed transactions. Moreover, the serialization should respect the realtime order: a transaction that completed before (in real time) another transaction started should appear first in the serialization.

By definition, opacity reduces correctness of a TM history to correctness of all its prefixes, and thus is prefix-closed and limit-closed. Thus, to verify that a history is opaque, one needs to verify that each of its prefixes is consistent with some global serialization. To simplify verification and explicitly introduce deferred-update semantics into a TM correctness criterion, we specify a general criterion of du-opacity [17, which requires the global serial execution to respect the deferred-update property. Informally, a du-opaque history must be indistinguishable from a totally-ordered history, with respect to which no transaction reads from a transaction that has not started committing. Assuming that in an infinite history, every transaction completes each of the operations it invoked, we prove that du-opacity is a safety property.

One may notice that the intended safety semantics does not require, as opacity does, that all transactions observe the same serial execution. As long as committed transactions constitute a serial execution and every transaction witnesses a consistent state, the execution can be considered "safe": no run-time error that cannot occur in a serial execution can happen. Two definitions in literature have adopted this approach [42, 83. We introduce "deferred-update" versions of these properties and discuss how the resulting properties relate to du-opacity.

\subsubsection{Complexity of transactional memory}

One may observe that a TM implementation that aborts or never commits any transaction is trivially safe, but not very useful. Thus, the TM implementation must satisfy some nontrivial liveness property specifying the conditions under which the transactional operations must return some response and a progress property specifying the conditions under which the transaction is allowed to abort.

Two properties considered important for TM performance are read invisibility [22] and disjoint-access parallelism [84]. Read invisibility may boost the concurrency of a TM implementation by ensuring that no reading transaction can cause any other transaction to abort. The idea of disjoint-access parallelism is to allow transactions that do not access the same data item to proceed independently of each other without memory contention.

We investigate the inherent complexities in terms of time and memory resources associated with implementing safe TMs that provide strong liveness and progress properties, possibly combined with attractive requirements like read invisibility and disjoint-access parallelism. Which classes of TM implementations are (im)possible to solve?

Blocking TMs. We begin by studying TM implementations that are blocking, in the sense that, a transaction may be delayed or aborted due to concurrent transactions.

- We prove that, even inherently sequential TMs, that allow a transaction to be aborted due to a concurrent transaction, incur significant complexity costs when combined with read invisibility and disjoint-access parallelism.

- We prove that, progressive TMs, that allow a transaction to be aborted only if it encounters a readwrite or write-write conflict with a concurrent transaction [60], may need to exclusively control a linear number of data items at some point in the execution.

- We then turn our focus to strongly progressive TMs 62 that, in addition to progressiveness, ensures that not all concurrent transactions conflicting over a single data item abort. We prove that in any strongly progressive TM implementation that accesses the shared memory with read, 
write and conditional primitives, such as compare-and-swap, the total number of remote memory references [13, 21] (RMRs) that take place in an execution in which $n$ concurrent processes perform transactions on a single data item might reach $\Omega(n \log n)$ in the worst-case.

- We show that, with respect to the amount of expensive synchronization patterns like compare-andswap instructions and memory barriers [16, 100, progressive implementations are asymptotically optimal. We use this result to establish a linear (in the transaction's data set size) separation between the worst-case transaction expensive synchronization complexity of progressive TMs and permissive TMs that allow a transaction to abort only if committing it would violate opacity.

Non-blocking TMs. Next, we focus on TMs that avoid using locks and rely on non-blocking synchronization: a prematurely halted transaction cannot not prevent other transactions from committing. Possibly the weakest non-blocking progress condition is obstruction-freedom [76, 80, stipulating that every transaction running in the absence of step contention, i.e., not encountering steps of concurrent transactions, must commit. In fact, several early TM implementations [51, 77, 98, 114, 117] satisfied obstruction-freedom. However, circa. 2005, several papers presented the case for a shift from TMs that provide obstruction-free TM-progress to lock-based progressive TMs [38, 39, 47]. They argued that lock-based TMs tend to outperform obstruction-free ones by allowing for simpler algorithms with lower complexity overheads. We prove the following lower bounds for obstruction-free TMs.

- Combining invisible reads with even weak forms of disjoint-access parallelism [23] in obstructionfree TMs is impossible,

- A read operation in a $n$-process obstruction-free TM implementation incurs $\Omega(n)$ memory stalls $[15$, 45 .

- A read-only transaction may need to perform a linear (in $n$ ) number of expensive synchronization patterns.

We then present a progressive TM implementation that beats all of these lower bounds, thus suggesting that the course correction from non-blocking (obstruction-free) TMs to blocking (progressive) TMs was indeed justified.

Partially non-blocking TMs. Lastly, we explore the costs of providing non-blocking progress to only a subset of transactions. Specifically, we require read-only transactions to commit wait-free, i.e., every transaction commits within a finite number of its steps, but updating transactions are guaranteed to commit only if they run in the absence of concurrency. We show that combining this kind of partial wait-freedom with read invisibility or disjoint-access parallelism comes with inherent costs. Specifically, we establish the following lower bounds for TMs that provide this kind of partial wait-freedom.

- This kind of partial wait-freedom equipped with invisible reads results in maintaining unbounded sets of versions for every data item.

- It is impossible to implement a strict form of disjoint-access parallelism [58].

- Combining with the weak form of disjoint-access parallelism means that a read-only transaction (with an arbitrarily large read set) must sometimes perform at least one expensive synchronization pattern per read operation in some executions.

\subsubsection{Hybrid transactional memory}

We turn our focus on Hybrid transactional memory (HyTM) [34, 36, 86, 96. The TM abstraction, in its original manifestation, augmented the processor's cache-coherence protocol and extended the CPU's instruction set with instructions to indicate which memory accesses must be transactional [78. Most popular TM designs, subsequent to the original proposal in [78, have implemented all the functionality in software [35, 51, 77, 98, 114. More recently, CPUs have included hardware extensions to support small transactions [1, 104, 108. Hardware transactions may be spuriously aborted due to several reasons: cache capacity overflow, interrupts etc. This has led to proposals for best-effort HyTMs in which the fast, but potentially unreliable hardware transactions are complemented with slower, but more reliable software 
transactions. However, the fundamental limitations of building a HyTM with nontrivial concurrency between hardware and software transactions are not well understood. Typically, hardware transactions usually employ code instrumentation techniques to detect concurrency scenarios and abort in the case of contention. But are there inherent instrumentation costs of implementing a HyTM, and what are the trade-offs between these costs ands provided concurrency, i.e., the ability of the HyTM to execute hardware and software transactions in parallel?

The thesis makes the following contributions which help determine the cost of concurrency in HyTMs.

- We propose a general model for HyTM implementations, which captures the notion of cached accesses as performed by hardware transactions, and precisely defines instrumentation costs in a quantifiable way.

- We derive lower and upper bounds in this model, which capture for the first time, an inherent trade-off on the degree of concurrency allowed between hardware and software transactions and the instrumentation overhead introduced on the hardware.

\subsubsection{Optimism for boosting concurrency}

Lock-based implementations are conventionally pessimistic in nature: the operations invoked by processes are not "abortable" and return only after they are successfully completed. The TM abstraction is a realization of optimistic concurrency control: speculatively execute transactions, abort and roll back on dynamically detected conflicts. But are optimistic implementations fundamentally better equipped to exploit concurrency than pessimistic ones?

We compare the amount of concurrency one can obtain by converting a sequential implementation of a data abstraction into a concurrent one using optimistic or pessimistic synchronization techniques. To establish fair comparison of such implementations, we introduce a new correctness criterion for concurrent implementations, called locally serializable linearizability, defined independently of the synchronization techniques they use.

We treat an implementation's concurrency as its ability to accept schedules of sequential operations from different processes. More specifically, we assume an external scheduler that defines which processes execute which steps of the corresponding sequential implementation in a dynamic and unpredictable fashion. This allows us to define concurrency provided by an implementation as the set of interleavings of steps of sequential operations (or schedules) it accepts, i.e., is able to effectively process. Then, the more schedules the implementation would accept without hampering correctness, the more concurrent it would be.

The thesis makes the following contributions.

- We provide a framework to analytically capture the inherent concurrency provided by two broad classes of synchronization techniques: pessimistic implementations that implement some form of mutual exclusion and optimistic implementations based on speculative executions.

- We show that, implementations based on pessimistic synchronization and "semantics-oblivious" TMs are suboptimal, in the sense that, there exist there exist simple schedules of the list-based set which cannot be accepted by any pessimistic or TM-based implementation. Specifically, we prove that TM-based implementations accept schedules of the list-based set that cannot be accepted by any pessimistic implementation. However, we also show pessimistic implementations of the list-based set which accept schedules that cannot be accepted by any TM-based implementation.

- We show that, there exists an optimistic implementation of the list-based set that is concurrency optimal, i.e., it accepts all correct schedules.

Our results suggest that "semantics-aware" optimistic implementations may be better suited to exploiting concurrency than their pessimistic counterparts. 


\subsection{Roadmap of the thesis}

We first define the TM model, the TM properties proposed in literature and the complexity metrics considered in Chapter 2, Chapter 3 is on safety for TMs. Chapter 4 is on the complexity of blocking TMs, non-blocking TMs that satisfy obstruction-freedom are covered in Chapter 5 and we present lower bounds for partially non-blocking TMs in Chapter 6. Chapter 7 is devoted to the study of hybrid TMs. In Chapter 8, we compare the relative abilities of optimistic and pessimistic synchronization techniques in exploiting concurrency. Chapter 9 presents closing comments and future directions. Viewed collectively, the results hopefully shine light on the foundations of the TM abstraction that is widely expected to be the Zeitgeist of the concurrent computational model. 



\section{2}

\section{Transactional Memory model}

All models are wrong, but some models are useful.

George Edward Pelham Box

In this chapter, we formalize the TM model and discuss some important TM properties proposed in literature. In Section 2.1 we formalize the specification of TMs. In Section 2.2, we introduce the basic TM-correctness property of strict serializability that we consider in the thesis. Sections 2.3 and 2.4 overview progress and liveness properties for TMs respectively and identifies the relations between them. Section 2.5 defines the notion of invisible reads while Section 2.6 is on disjoint-access parallelism. Finally, in Section 2.7. we introduce some of the complexity metrics considered in the thesis.

\subsection{TM interface and TM implementations}

In this section, we first describe the shared memory model of computation and then introduce the TM abstraction.

The shared memory model. The thesis considers the standard asynchronous shared memory model of computation in which a set of $n \in \mathbb{N}$ processes (that may fail by crashing), communicate by applying operations on shared objects [15]. An object is an instance of an abstract data type. An abstract data type $\tau$ is a mealy machine that is specified as a tuple $\left(\Phi, \Gamma, Q, q_{0}, \delta\right)$ where $\Phi$ is a set of operations, $\Gamma$ is a set of responses, $Q$ is a set of states, $q_{0} \in Q$ is an initial state and $\delta \subseteq Q \times \Phi \times Q \times \Gamma$ is a transition relation that determines, for each state and each operation, the set of possible resulting states and produced responses [6]. Here, $\left(q, \pi, q^{\prime}, r\right) \in \delta$ implies that when an operation $\pi \in \Phi$ is applied on an object of type $\tau$ in state $q$, the object moves to state $q^{\prime}$ and returns response $r$.

An implementation of an object type $\tau$ provides a specific data-representation of $\tau$ that is realized by processes applying primitives on shared base objects, each of which is assigned an initial value. In order to implement an object, processes are provided with an algorithm, which is a set of deterministic statemachines, one for each process. In the thesis, we use the term primitive to refer to operations on base objects and reserve the term operation for the object that is implemented from the base objects. 
A primitive is a generic atomic read-modify-write $(\mathrm{rmw})$ procedure applied to a base object [45, 73]. It is characterized by a pair of functions $\langle g, h\rangle$ : given the current state of the base object, $g$ is an update function that computes its state after the primitive is applied, while $h$ is a response function that specifies the outcome of the primitive returned to the process. A rmw primitive is trivial if it never changes the value of the base object to which it is applied. Otherwise, it is nontrivial. An rmw primitive $\langle g, h\rangle$ is conditional if there exists $v, w$ such that $g(v, w)=v$ and there exists $v, w$ such that $g(v, w) \neq v[50$.

Read is an example of a trivial rmw primitive that takes no input arguments: when applied to a base object with value $v$, the update function leaves the state of the base object unchanged and the response function returns the value $v$. Write is an example of a nontrivial $\mathrm{rmw}$ primitive that takes an input argument $v^{\prime}$ : when applied to a base object with value $v$, its update function changes the value of the base object to $v^{\prime}$ and its response function returns ok. Compare-and-swap is an example of a nontrivial conditional $\mathrm{rmw}$ primitive: its update function receives an input argument $\langle$ old, new $\rangle$ and changes the value $v$ of the base object to which it is applied iff $v=$ old. Load-linked/store-conditional is another example of a nontrivial conditional rmw primitive: the load-linked primitive executed by some process $p_{i}$ returns the value of the base object to which it is applied and the store-conditional primitive's update function receives an input new and atomically changes the value of the base object to new iff the base object has not been updated by any other process since the load-linked event by $p_{i}$. Fetch-and-add is an example of a nontrivial $\mathrm{rmw}$ primitive that is not conditional: its update function applied to base object with an integer value $v$ takes an integer $w$ as input and changes the value of the base object to $v+w$.

Transactional memory (TM). Transactional memory allows a set of data items (called t-objects) to be accessed via transactions. Every transaction $T_{k}$ has a unique identifier $k$. We make no assumptions on the size of a t-object, i.e., the cardinality on the set $V$ of possible values a t-object can store. A transaction $T_{k}$ may contain the following t-operations, each being a matching pair of an invocation and a response: $\operatorname{read}_{k}(X)$ returns a value in $V$, denoted $\operatorname{read}_{k}(X) \rightarrow v$, or a special value $A_{k} \notin V$ (abort); write $_{k}(X, v)$, for a value $v \in V$, returns ok or $A_{k} ; \operatorname{try} C_{k}$ returns $C_{k} \notin V$ (commit) or $A_{k}$. As we show in the subsequent Section 2.2 , we can specify TM as an abstract data type.

Note that a TM interface may additionally provide a start $t_{k}$ t-operation that returns ok or $A_{k}$, which is the first t-operation transaction $T_{k}$ must invoke, or a $\operatorname{try} A_{k}$ t-operation that returns $A_{k}$. However, the actions performed inside the start $_{k}$ may be performed as part of the first t-operation performed by the transaction. The $\operatorname{try} A_{k}$ t-operation allows the user application to explicitly abort a transaction and can be useful, but since each of the individual t-read or t-write are allowed to abort, the try $A_{k}$ t-operation provides no additional expressive power to the TM interface. Thus, for simplicity, we do not incorporate these t-operations in our TM specification.

TM implementations. A TM implementation provides processes with algorithms for implementing read $_{k}$, write $_{k}$ and $\operatorname{try} C_{k}()$ of a transaction $T_{k}$ by applying primitives from a set of shared base objects, each of which is assigned an initial value. We assume that a process starts a new transaction only after its previous transaction has committed or aborted.

In the rest of this section, we define the terms specifically in the context of TM implementations, but they may be used analogously in the context of any concurrent implementation of an abstract data type.

Executions and configurations. An event of a process $p_{i}$ (sometimes we say step of $p_{i}$ ) is an invocation or response of an operation performed by $p_{i}$ or a rmw primitive $\langle g, h\rangle$ applied by $p_{i}$ to a base object $b$ along with its response $r$ (we call it a $r m w$ event and write $(b,\langle g, h\rangle, r, i)$ ).

A configuration (of an implementation) specifies the value of each base object and the state of each process. The initial configuration is the configuration in which all base objects have their initial values and all processes are in their initial states.

An execution fragment is a (finite or infinite) sequence of events. An execution of an implementation $M$ is an execution fragment where, starting from the initial configuration, each event is issued according to $M$ and each response of a rmw event $(b,\langle g, h\rangle, r, i)$ matches the state of $b$ resulting from all preceding events. An execution $E \cdot E^{\prime}$ denotes the concatenation of $E$ and execution fragment $E^{\prime}$, and we say that $E^{\prime}$ is an extension of $E$ or $E^{\prime}$ extends $E$. 
Let $E$ be an execution fragment. For every transaction (resp., process) identifier $k, E \mid k$ denotes the subsequence of $E$ restricted to events of transaction $T_{k}$ (resp., process $p_{k}$ ). If $E \mid k$ is non-empty, we say that $T_{k}$ (resp., $p_{k}$ ) participates in $E$, else we say $E$ is $T_{k}$-free (resp., $p_{k}$-free). Two executions $E$ and $E^{\prime}$ are indistinguishable to a set $\mathcal{T}$ of transactions, if for each transaction $T_{k} \in \mathcal{T}, E\left|k=E^{\prime}\right| k$. A TM history is the subsequence of an execution consisting of the invocation and response events of t-operations. Two histories $H$ and $H^{\prime}$ are equivalent if $\operatorname{txns}(H)=\operatorname{txns}\left(H^{\prime}\right)$ and for every transaction $T_{k} \in \operatorname{txns}(H), H\left|k=H^{\prime}\right| k$.

Data sets of transactions. The read set (resp., the write set) of a transaction $T_{k}$ in an execution $E$, denoted $\operatorname{Rset}_{E}\left(T_{k}\right)$ (resp., Wset ${ }_{E}\left(T_{k}\right)$ ), is the set of t-objects that $T_{k}$ reads (resp., writes to) in $E$. More specifically, if $E$ contains an invocation of $\operatorname{read}_{k}(X)$ (resp., write $_{k}(X, v)$ ), we say that $X \in \operatorname{Rset}_{E}\left(T_{k}\right)$ (resp., $W \operatorname{set}_{E}\left(T_{k}\right)$ ) (for brevity, we sometimes omit the subscript $E$ from the notation). The data set of $T_{k}$ is $\operatorname{Dset}\left(T_{k}\right)=\operatorname{Rset}\left(T_{k}\right) \cup W \operatorname{set}\left(T_{k}\right)$. A transaction is called read-only if $\operatorname{Rset}\left(T_{k}\right) \neq \emptyset \wedge W \operatorname{set}\left(T_{k}\right)=\emptyset$; write-only if $W \operatorname{set}\left(T_{k}\right) \neq \emptyset \wedge \operatorname{Rset}\left(T_{k}\right)=\emptyset$ and updating if $W \operatorname{set}\left(T_{k}\right) \neq \emptyset$. Note that, in our TM model, the data set of a transaction is not known apriori, i.e., at the start of the transaction and it is identifiable only by the set of data items the transaction has invoked a read or write on in the given execution.

Transaction orders. Let $\operatorname{txns}(E)$ denote the set of transactions that participate in $E$. In an infinite history $H$, we assume that each $T_{k} \in \operatorname{txns}(H), H \mid k$ is finite; i.e., transactions do not issue an infinite number of t-operations. An execution $E$ is sequential if every invocation of a t-operation is either the last event in the history $H$ exported by $E$ or is immediately followed by a matching response. We assume that executions are well-formed, i.e., for all $T_{k}, E \mid k$ begins with the invocation of a t-operation, is sequential and has no events after $A_{k}$ or $C_{k}$. A transaction $T_{k} \in \operatorname{txns}(E)$ is complete in $E$ if $E \mid k$ ends with a response event. The execution $E$ is complete if all transactions in $\operatorname{txns}(E)$ are complete in $E$. A transaction $T_{k} \in \operatorname{txns}(E)$ is t-complete if $E \mid k$ ends with $A_{k}$ or $C_{k}$; otherwise, $T_{k}$ is $t$-incomplete. $T_{k}$ is committed (resp., aborted) in $E$ if the last event of $T_{k}$ is $C_{k}$ (resp., $A_{k}$ ). The execution $E$ is $t$-complete if all transactions in $\operatorname{txns}(E)$ are t-complete.

For transactions $\left\{T_{k}, T_{m}\right\} \in$ txns $(E)$, we say that $T_{k}$ precedes $T_{m}$ in the real-time order of $E$, denoted $T_{k} \prec_{E}^{R T} T_{m}$, if $T_{k}$ is t-complete in $E$ and the last event of $T_{k}$ precedes the first event of $T_{m}$ in $E$. If neither $T_{k} \prec_{E}^{R T} T_{m}$ nor $T_{m} \prec_{E}^{R T} T_{k}$, then $T_{k}$ and $T_{m}$ are concurrent in $E$. An execution $E$ is $t$-sequential if there are no concurrent transactions in $E$.

Latest written value and legality. Let $H$ be a t-sequential history. For every operation $\operatorname{read}_{k}(X)$ in $H$, we define the latest written value of $X$ as follows: if $T_{k}$ contains a write $_{k}(X, v)$ preceding $\operatorname{read}_{k}(X)$, then the latest written value of $X$ is the value of the latest such write to $X$. Otherwise, the latest written value of $X$ is the value of the argument of the latest write $_{m}(X, v)$ that precedes read $_{k}(X)$ and belongs to a committed transaction in $H$. (This write is well-defined since $H$ starts with $T_{0}$ writing to all t-objects.)

We say that read $_{k}(X)$ is legal in a t-sequential history $H$ if it returns the latest written value of $X$, and $H$ is legal if every $\operatorname{read}_{k}(X)$ in $H$ that does not return $A_{k}$ is legal in $H$.

We also assume, for simplicity, that the user application invokes a $\operatorname{read}_{k}(X)$ at most once within a transaction $T_{k}$. This assumption incurs no loss of generality, since a repeated read can be assigned to return a previously returned value without affecting the history's legality.

Contention. We say that a configuration $C$ after an execution $E$ is quiescent (resp., $t$-quiescent) if every transaction $T_{k} \in \operatorname{txns}(E)$ is complete (resp., t-complete) in $C$. If a transaction $T$ is incomplete in an execution $E$, it has exactly one enabled event, which is the next event the transaction will perform according to the TM implementation. Events $e$ and $e^{\prime}$ of an execution $E$ contend on a base object $b$ if they are both events on $b$ in $E$ and at least one of them is nontrivial (the event is trivial (resp., nontrivial) if it is the application of a trivial (resp., nontrivial) primitive).

We say that $T$ is poised to apply an event $e$ after $E$ if $e$ is the next enabled event for $T$ in $E$. We say that transactions $T$ and $T^{\prime}$ concurrently contend on $b$ in $E$ if they are poised to apply contending events on $b$ after $E$. 
We say that an execution fragment $E$ is step contention-free for t-operation $o p_{k}$ if the events of $E \mid o p_{k}$ are contiguous in $E$. We say that an execution fragment $E$ is step contention-free for $T_{k}$ if the events of $E \mid k$ are contiguous in $E$. We say that $E$ is step contention-free if $E$ is step contention-free for all transactions that participate in $E$.

\subsection{TM-correctness}

Correctness for TMs is specified as a safety property on TM histories [10, 97, 105]. In this section, we introduce the popular TM-correctness condition strict serializability [106]: all committed transactions appear to execute sequentially in some total order respecting the real-time transaction orders. We then explain how strict serializability is related to linearizability [81.

In the thesis, we only consider TM-correctness conditions like strict serializability and its restrictions. We formally define strict serializability below, but other TM-correctness conditions studied in the thesis can be found in Chapter 3 .

First, we define how to derive a t-complete history from a t-incomplete one.

Definition 2.1 (Completions). Let $H$ be a history. A completion of $H$, denoted $\bar{H}$, is a history derived from $H$ as follows:

- First, for every transaction $T_{k} \in \operatorname{txns}(H)$ with an incomplete t-operation op $p_{k}$ in $H$, if $o p_{k}=$ read $_{k} \vee$ write $_{k}$, insert $A_{k}$ somewhere after the invocation of op $p_{k}$; otherwise, if op $p_{k}=\operatorname{try} C_{k}()$, insert $C_{k}$ or $A_{k}$ somewhere after the last event of $T_{k}$.

- After all transactions are complete, for every transaction $T_{k}$ that is not $t$-complete, insert try $C_{k} \cdot A_{k}$ after the last event of transaction $T_{k}$.

Definition 2.2 (Strict serializability). A finite history $H$ is serializable if there is a legal t-complete t-sequential history $S$, such that there is a completion $\bar{H}$ of $H$, such that $S$ is equivalent to cseq $(\bar{H})$, where cseq $(\bar{H})$ is the subsequence of $\bar{H}$ reduced to committed transactions in $\bar{H}$.

We refer to $S$ as a serialization of $H$.

We say that $H$ is strictly serializable if there exists a serialization $S$ of $H$ such that for any two transactions $T_{k}, T_{m} \in$ txns $(H)$, if $T_{k} \prec_{H}^{R T} T_{m}$, then $T_{k}$ precedes $T_{m}$ in $S$.

In general, given a TM-correctness condition $C$, we say that a TM implementation $M$ satisfies $C$ if every execution of $M$ satisfies $C$.

Strict serializability as linearizability. We now show we can specify TM as an abstract data type.

The sequential specification of a TM is specified as follows:

1. $\Phi$ is the set of all transactions $\left\{T_{i}\right\}_{i \in \mathbb{N}}$

2. $\Gamma$ is the set of incommensurate vectors $\left\{\left[r_{1}, \ldots, r_{i}\right]\right\} ; i \in \mathbb{N}$; where each $r_{j} ; 1 \leq j \leq i-1 \in\{v \in$ $V, A, o k\}$ and $r_{i} \in\{A, C\}$

3. The state of TM is a vector of the state of each t-object $X_{m}$. The state of a t-object $X_{m}$ is a value $v_{m} \in V$ of $X_{m}$. Thus, $Q \subseteq\left\{\left[v_{1}^{i}, \ldots, v_{m}^{i}, \ldots\right]\right\}$; where each $v_{m}^{i} \in V$

4. $q_{0} \in Q=\left[o v_{1}, \ldots, o v_{m}, \ldots\right]$, where each $o v_{m} \in V$

5. $\delta$ is defined as follows: Let $T_{k}$ be a transaction applied to the TM in state $q=\left[v_{1}, \ldots, v_{m}, \ldots\right]$.

- For every $X \in R \operatorname{Ret}\left(T_{k}\right)$, the response of $\operatorname{read}_{k}(X)$ is defined as follows: If $T_{k}$ contains a write $_{k}(X, v)$ prior to read $(X)$, then the response is $v$; else the response is the current state of $X$.

- For every $X \in W \operatorname{set}\left(T_{k}\right)$, the response of write $_{k}(X, v)$ is $o k$. 
- Transaction $T_{k}$ returns the response $C$ in which case the TM moves to state $q^{\prime}$ defined as follows: every $X_{j} \in W \operatorname{set}\left(T_{k}\right)$ to which $T_{k}$ writes values $n v_{j}, q^{\prime}[j]=n v_{j}$; else if $X_{j} \notin$ $W \operatorname{set}\left(T_{k}\right), q^{\prime}[j]$ is unchanged. Otherwise, $T_{k}$ returns the response $A$ in which case $q^{\prime}=q$.

In general, the correctness of an implementation of a data type is commonly captured by the criterion of linearizability. In the TM context, a t-complete history $H$ is linearizable with respect to the TM type if there exists a t-sequential history $S$ equivalent to $H$ such that (1) $S$ respects the real-time ordering of transactions in $H$ and (2) $S$ is consistent with the sequential specification of TM.

The following lemma, which illustrates the similarity between strict serializability and linearizability with respect to the TM type, is now immediate.

Lemma 2.1. Let $H$ be any t-complete history. Then, $H$ is strictly serializable iff $H$ is linearizable with respect to the TM type.

\subsection{TM-progress}

One may notice that a TM implementation that forces, in every execution to abort every transaction is trivially strictly serializable, but not very useful. A TM-progress condition specifies the conditions under which a transaction is allowed to abort. Technically, a TM-progress condition specified this way is a safety property since it can be violated in a finite execution (cf. Chapter 3 for details on safety properties).

Ideally, a TM-progress condition must provide non-blocking progress, in the sense that a prematurely halted transaction cannot prevent all other transactions from committing. Such a TM-progress condition is also said to be lock-free since it cannot be achieved by use of locks and mutual-exclusion. A nonblocking TM-progress condition is considered useful in asynchronous systems with process failures since it prevents the TM implementation from deadlocking (processes wait infinitely long without committing their transactions).

Obstruction-freedom. Perhaps, the weakest non-blocking TM-progress condition is obstructionfreedom, which stipulates that a transaction may be aborted only if it encounters steps of a concurrent transaction 80 .

Definition 2.3 (Obstruction-free (OF) TM-progress). We say that a TM implementation $M$ provides obstruction-free (OF) TM-progress if for every execution $E$ of $M$, if any transaction $T_{k} \in \operatorname{txns}(E)$ returns $A_{k}$ in $E$, then $E$ is not step contention-free for $T_{k}$.

We now survey the popular blocking TM-progress properties proposed in literature. Intuitively, unlike non-blocking TM-progress conditions that adapt to step contention, a blocking TM-progress condition allows a transaction to be aborted due to overlap contention.

Minimal progressiveness. Intuitively, the most basic TM-progress condition is one which provide only sequential TM-progress, i.e., a transaction may be aborted due to a concurrent transaction. In literature, this is referred to as minimal progressiveness [62].

Definition 2.4 (Minimal progressiveness). We say that a TM implementation $M$ provides minimal progressive TM-progress (or minimal progressiveness) if for every execution $E$ of $M$ and every transaction $T_{k} \in \operatorname{txns}(E)$ that returns $A_{k}$ in $E$, there exists a transaction $T_{m} \in \operatorname{txns}(E)$ that is concurrent to $T_{k}$ in $E$ [62].

Given TM conditions $C_{1}$ and $C_{2}$, if every TM implementation that satisfies $C_{1}$ also satisfies $C_{2}$, but the converse is not true, we say that $C_{2} \ll C_{1}$.

Observation 2.2. Minimal progressiveness $\ll$ Obstruction-free. 
Proof. Clearly, every TM implementation that satisfies obstruction-freedom also satisfies minimal progressiveness, but the converse is not true. Consider any execution of a TM implementation $M$ in which a transaction $T$ run step contention-free. If $M$ is minimally progressive, then $T$ may be aborted in such an execution since $T$ may be concurrent with another transaction. However, if $M$ satisfies obstructionfreedom, $T$ cannot be aborted in such an execution.

Progressiveness. In contrast to the "single-lock" minimal progressive TM-progress condition (also referred to as sequential TM-progress in the thesis), state-of-the-art TM implementations allow a transaction to abort only if it encounters a conflict on a t-object with a concurrent transaction.

Definition 2.5 (Conflicts). We say that transactions $T_{i}, T_{j}$ conflict in an execution $E$ on a t-object $X$ if $T_{i}$ and $T_{j}$ are concurrent in $E$ and $X \in \operatorname{Dset}\left(T_{i}\right) \cap \operatorname{Dset}\left(T_{j}\right)$, and $X \in W \operatorname{set}\left(T_{i}\right) \cup W \operatorname{set}\left(T_{j}\right)$.

Definition 2.6 (Progressiveness). A TM implementation M provides progressive TM-progress (or progressiveness) if for every execution $E$ of $M$ and every transaction $T_{i} \in \operatorname{txns}(E)$ that returns $A_{i}$ in $E$, there exists a transaction $T_{k} \in \operatorname{txns}(E)$ such that $T_{k}$ and $T_{i}$ conflict in $E$ [62].

Note that progressiveness is incomparable to obstruction-freedom.

Observation 2.3. Progressiveness $\nless$ Obstruction-free and Obstruction-free $\nless$ Progressiveness.

Proof. We can show that there exists an execution exported by an obstruction-free TM, but not by any progressive TM and vice-versa.

Consider a t-read $X$ by a transaction $T$ that runs step contention-free from a configuration that contains an incomplete write to $X$. Weak progressiveness does not preclude $T$ from being aborted in such an execution. Obstruction-free TMs however, must ensure that $T$ must complete its read of $X$ without blocking or aborting in such executions. On the other hand, weak progressiveness requires two nonconflicting transactions to not be aborted even in executions that are not step contention-free; but this is not guaranteed by obstruction-freedom.

In general, progressive TMs (including the ones described in the thesis) satisfy the following stronger definition: for every transaction $T_{i} \in \operatorname{txns}(E)$ that returns $A_{i}$ in an execution $E$, there exists prefix $E^{\prime}$ of $E$ and a transaction $T_{k} \in \operatorname{txns}\left(E^{\prime}\right)$ such that $T_{k}$ and $T_{i}$ conflict in $E$. However, for the lower bound results stated in the thesis, we stick to Definition 2.6.

Strong progressiveness. One may observe that the definition of progressiveness does not preclude two conflicting transactions (over a single t-object) from each being aborted. Thus, we study a stronger notion of progressiveness called strong progressiveness [62.

Let $\operatorname{CObj}_{E}\left(T_{i}\right)$ denote the set of t-objects over which transaction $T_{i} \in \operatorname{txns}(H)$ conflicts with any other transaction in an execution $E$, i.e., $X \in C O b j_{E}\left(T_{i}\right)$, iff there exist transactions $T_{i}$ and $T_{k}$ that conflict on $X$ in $E$. Let $Q \subseteq \operatorname{txns}(E)$ and $\operatorname{CObj}_{E}(Q)=\bigcup_{T_{i} \in Q} \operatorname{CObj}_{E}\left(T_{i}\right)$.

Let $C \operatorname{Trans}(E)$ denote the set of non-empty subsets of txns $(E)$ such that a set $Q$ is in $C \operatorname{Trans}(E)$ if no transaction in $Q$ conflicts with a transaction not in $Q$.

Definition 2.7 (Strong progressiveness). A TM implementation $M$ is strongly progressive if $M$ is weakly progressive and for every execution $E$ of $M$ and for every set $Q \in C \operatorname{Trans}(E)$ such that $\left|C O b j_{E}(Q)\right| \leq 1$, some transaction in $Q$ is not aborted in $E$ [62].

The above definitions imply:

Corollary 2.4. Minimal progressiveness (sequential TM-progress) $\ll$ Progressiveness $\ll$ Strong progressiveness. 
Mv-permissiveness. Perelman et al. introduced the notion of mv-permissiveness, a TM-progress property designed to prevent read-only transactions from being aborted.

Definition 2.8 (Mv-permissiveness). A TM implementation $M$ is mv-permissive if for every execution $E$ of $M$ and for every transaction $T_{k} \in \operatorname{txns}(E)$ that returns $A_{k}$ in $E$, we have that $W \operatorname{set}\left(T_{k}\right) \neq \emptyset$ and there exists an updating transaction $T_{m} \in \operatorname{txns}(E)$ such that $T_{k}$ and $T_{m}$ conflict in $E$.

We observe that mv-permissiveness is strictly stronger than progressiveness, but incomparable to strong progressiveness.

Observation 2.5. Progressiveness $\ll M v$-permissiveness.

Proof. Since mv-permissive TMs allow a transaction to be aborted only on read-write conflicts, they also satisfy progressiveness. But the converse is not true. Consider an execution in which a read-only transaction $T_{i}$ that runs concurrently with a conflicting updating transaction $T_{j}$. By the definition of progressiveness, both $T_{i}$ and $T_{j}$ may be aborted in such an execution. However, a mv-permissive TM would not allow $T_{i}$ to be aborted since it is read-only.

Observation 2.6. Strong progressiveness $\nless M v$-permissiveness and Mv-permissiveness $\nless$ Strong progressiveness.

Proof. Consider an execution in which a read-only transaction $T_{i}$ that runs concurrently with an updating transaction $T_{j}$ such that $T_{i}$ and $T_{j}$ conflict on at least two t-objects. By the definition of strong progressiveness, both $T_{i}$ and $T_{j}$ may be aborted in such an execution. However, a mv-permissive TM would not allow $T_{i}$ to be aborted since it is read-only.

On the other hand, consider an execution in which two updating transactions $T_{i}$ and $T_{j}$ that conflict on a single t-object. A mv-permissive TM allows both $T_{i}$ and $T_{j}$ to be aborted, but strong progressiveness ensures that at least one of $T_{i}$ or $T_{j}$ is not aborted in such an execution.

\subsection{TM-liveness}

Observe that a TM-progress condition only specifies the conditions under which a transaction is aborted, but does not specify the conditions under which it must commit. For instance, the OF TM-progress condition specifies that a transaction $T$ may be aborted only in executions that are not step contentionfree for $T$, but does not guarantee that $T$ is committed in a step contention-free execution. Thus, in addition to a progress condition, we must stipulate a liveness [10, 97, condition.

We now define the TM-liveness conditions considered in the thesis.

Definition 2.9 (Sequential TM-liveness). A TM implementation $M$ provides sequential TM-liveness if for every finite execution $E$ of $M$, and every transaction $T_{k}$ that runs $t$-sequentially and applies the

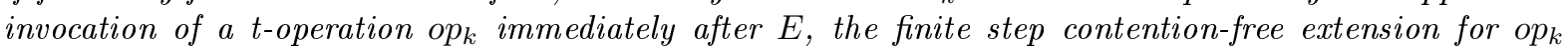
contains a response.

Definition 2.10 (Interval contention-free (ICF) TM-liveness). A TM implementation $M$ provides interval contention-free (ICF) TM-liveness if for every finite execution $E$ of $M$ such that the configuration after $E$ is quiescent, and every transaction $T_{k}$ that applies the invocation of a t-operation op immediately after $E$, the finite step contention-free extension for op $p_{k}$ contains a response.

Definition 2.11 (Starvation-free TM-liveness). A TM implementation $M$ provides starvation-free TMliveness if in every execution of $M$, each t-operation eventually returns a matching response, assuming that no concurrent t-operation stops indefinitely before returning.

Definition 2.12 (Obstruction-free (OF) TM-liveness). A TM implementation $M$ provides obstructionfree (OF) TM-liveness if for every finite execution $E$ of $M$, and every transaction $T_{k}$ that applies the invocation of a t-operation op $_{k}$ immediately after $E$, the finite step contention-free extension for op ${ }_{k}$ contains a matching response. 
Definition 2.13 (Wait-free (WF) TM-liveness). A TM implementation $M$ provides wait-free (WF) TM-liveness if in every execution of $M$, every t-operation returns a response in a finite number of its steps.

The following observations are immediate from the definitions:

Observation 2.7. Sequential TM-liveness $\ll I C F$ TM-liveness $\ll$ OF TM-liveness $\ll$ WF TM-liveness and Starvation-free TM-liveness $\ll W F$ TM-liveness.

Since ICF TM-liveness guarantees that a t-operation returns a response if there is no other concurrent t-operation, we have:

Observation 2.8. ICF TM-liveness $\ll$ Starvation-free TM-liveness.

However, we observe that OF TM-liveness and starvation-free TM-liveness are incomparable.

Observation 2.9. Starvation-free TM-liveness $\nless$ OF TM-liveness and OF TM-liveness $\nless$ Starvationfree TM-liveness.

Proof. Consider the step contention-free execution of t-operation $o p_{k}$ concurrent with t-operation $o p_{m}$ : $o p_{k}$ must return a matching response within a finite number of its steps, but this is not necessarily ensured by starvation-free TM-liveness ( $o p_{m}$ may be delayed indefinitely). On the other hand, in executions where two concurrent t-operations $o p_{k}$ and $o p_{k}$ encounter step contention, but neither stalls indefinitely, both must return matching responses. But this is not guaranteed by OF TM-liveness.

\subsection{Invisible reads}

In this section, we introduce the notion of invisible reads that intuitively ensures that a reading transaction does not cause a concurrent transaction to abort. Since most TM worklods are believed to be read-dominated, this is considered to be an important TM property for performance [24, 63].

Invisible reads. Informally, in a TM using invisible reads, a transaction cannot reveal any information about its read set to other transactions. Thus, given an execution $E$ and some transaction $T_{k}$ with a non-empty read set, transactions other than $T_{k}$ cannot distinguish $E$ from an execution in which $T_{k}$ 's read set is empty. This prevents TMs from applying nontrivial primitives during t-read operations and from announcing read sets of transactions during tryCommit. Most popular TM implementations like TL2 [38] and NOrec [35] satisfy this property.

Definition 2.14 (Invisible reads [22]). We say that a TM implementation $M$ uses invisible reads if for every execution $E$ of $M$ :

- for every read-only transaction $T_{k} \in$ txns $(E)$, no event of E|k is nontrivial in $E$,

- for every updating transaction $T_{k} \in \operatorname{txns}(E) ; \operatorname{Rset}_{E}\left(T_{k}\right) \neq \emptyset$, there exists an execution $E^{\prime}$ of $M$ such that

$-\operatorname{Rset}_{E^{\prime}}\left(T_{k}\right)=\emptyset$,

$-\operatorname{txns}(E)=\operatorname{txns}\left(E^{\prime}\right)$ and $\forall T_{m} \in \operatorname{txns}(E) \backslash\left\{T_{k}\right\}: E\left|m=E^{\prime}\right| m$

- for any two transactions $T_{i}, T_{j} \in \operatorname{txns}(E)$, if the last event of $T_{i}$ precedes the first event of $T_{j}$ in $E$, then the last event of $T_{i}$ precedes the first event of $T_{j}$ in $E^{\prime}$.

Weak invisible reads. We introduce the notion of weak invisible reads that prevents t-read operations from applying nontrivial primitives only in the absence of concurrent transactions. Specifically, weak read invisibility allows t-read operations of a transaction $T$ to be "visible", i.e., write to base objects, only if $T$ is concurrent with another transaction. 
Definition 2.15 (Weak invisible reads). For any execution $E$ and any -operation $\pi_{k}$ invoked by some transaction $T_{k} \in$ txns $(E)$, let $E \mid \pi_{k}$ denote the subsequence of $E$ restricted to events of $\pi_{k}$ in $E$.

We say that a TM implementation $M$ satisfies weak invisible reads if for any execution $E$ of $M$ and every transaction $T_{k} \in \operatorname{txns}(E) ; \operatorname{Rset}\left(T_{k}\right) \neq \emptyset$ that is not concurrent with any transaction $T_{m} \in \operatorname{txns}(E)$, $E \mid \pi_{k}$ does not contain any nontrivial events, where $\pi_{k}$ is any t-read operation invoked by $T_{k}$ in $E$.

For example, the popular TM implementation DSTM [77] satisfies weak invisible reads, but not invisible reads. Algorithm 5.1 in Chapter 4 depicts a TM implementation that is based on DSTM satisfying weak invisible reads, but not the stronger definition of invisible reads.

\subsection{Disjoint-access parallelism (DAP)}

The notion of disjoint-access parallelism (DAP) [84] is considered important in the TM context since it allows two transactions accessing unrelated t-objects to execute without memory contention. In this section, we preview the DAP definitions proposed in literature and identify the relations between them.

Strict data-partitioning. Let $E \mid X$ denote the subsequence of the execution $E$ derived by removing all events associated with t-object $X$. A TM implementation $M$ is strict data-partitioned [62, if for every t-object $X$, there exists a set of base objects $\operatorname{Base}_{M}(X)$ such that

- for any two t-objects $X_{1}, X_{2} ;$ Base $_{M}\left(X_{1}\right) \cap$ Base $_{M}\left(X_{2}\right)=\emptyset$,

- for every execution $E$ of $M$ and every transaction $T \in \operatorname{txns}(E)$, every base object accessed by $T$ in $E$ is contained in $\operatorname{Base}_{M}(X)$ for some $X \in \operatorname{Dset}(T)$

- for all executions $E$ and $E^{\prime}$ of $M$, if $E|X=E| X$ for some t-object $X$, then the configurations after $E$ and $E^{\prime}$ only differ in the states of the base objects in $\operatorname{Base}_{M}(X)$.

Strict disjoint-access parallelism. A TM implementation $M$ is strictly disjoint-access parallel (strict $D A P$ ) if, for all executions $E$ of $M$, and for all transactions $T_{i}$ and $T_{j}$ that participate in $E, T_{i}$ and $T_{j}$ contend on a base object in $E$ only if $\operatorname{Dset}\left(T_{i}\right) \cap \operatorname{Dset}\left(T_{j}\right) \neq \emptyset[62]$.

Proposition 2.1. Strict DAP Strict data-partitioning.

Proof. Let $M$ be any strict data-partitioned TM implementation. Then, $M$ is also strict DAP. Indeed, since any two transactions accessing mutually disjoint data sets in a strict data-partitioned implementation cannot access a common base object in any execution $E$ of $M, E$ also ensures that any two transactions contend on the same base object in $E$ only if they access a common t-object.

Consider the following execution $E$ of a strict DAP TM implementaton $M$ that begins with two transactions $T_{1}$ and $T_{2}$ that access disjoint data sets in $E$. A strict data-partitioned TM implementation would preclude transactions $T_{1}$ and $T_{2}$ from accessing the same base object, but a strict DAP TM implementation does not preclude this possibility.

We now describe two relaxations of strict DAP. For the formal definitions, we introduce the notion of a conflict graph which captures the dependency relation among t-objects accessed by transactions.

Read-write (RW) disjoint-access parallelism. Informally, read-write (RW) DAP means that two transactions can contend on a common base object only if their data sets are connected in the conflict graph, capturing write-set overlaps among all concurrent transactions.

We denote by $\tau_{E}\left(T_{i}, T_{j}\right)$, the set of transactions $\left(T_{i}\right.$ and $T_{j}$ included) that are concurrent to at least one of $T_{i}$ and $T_{j}$ in an execution $E$.

Let $\tilde{G}\left(T_{i}, T_{j}, E\right)$ be an undirected graph whose vertex set is $\bigcup_{T \in \tau_{E}\left(T_{i}, T_{j}\right)} \operatorname{Dset}(T)$ and there is an edge between t-objects $X$ and $Y$ iff there exists $T \in \tau_{E}\left(T_{i}, T_{j}\right)$ such that $\{X, Y\} \in W$ set $(T)$. We say that 
$T_{i}$ and $T_{j}$ are read-write disjoint-access in $E$ if there is no path between a t-object in $\operatorname{Dset}\left(T_{i}\right)$ and a t-object in $\operatorname{Dset}\left(T_{j}\right)$ in $\tilde{G}\left(T_{i}, T_{j}, E\right)$. A TM implementation $M$ is read-write disjoint-access parallel (RW $D A P)$ if, for all executions $E$ of $M$, transactions $T_{i}$ and $T_{j}$ contend on the same base object in $E$ only if $T_{i}$ and $T_{j}$ are not read-write disjoint-access in $E$ or there exists a t-object $X \in \operatorname{Dset}\left(T_{i}\right) \cap \operatorname{Dset}\left(T_{j}\right)$.

Proposition 2.2. $R W D A P \ll$ Strict DAP.

Proof. From the definitions, it is immediate that every strict DAP TM implementation satisfies RW DAP.

But the converse is not true (Algorithm 5.1 describes a TM implementation that satisfies RW and weak DAP, but not strict DAP). Consider the following execution $E$ of a weak DAP or RW DAP TM implementaton $M$ that begins with the t-incomplete execution of a transaction $T_{0}$ that accesses t-objects $X$ and $Y$, followed by the step contention-free executions of two transactions $T_{1}$ and $T_{2}$ which access $X$ and $Y$ respectively. Transactions $T_{1}$ and $T_{2}$ may contend on a base object since there is a path between $X$ and $Y$ in $G\left(T_{1}, T_{2}, E\right)$. However, a strict DAP TM implementation would preclude transactions $T_{1}$ and $T_{2}$ from contending on the same base object since $\operatorname{Dset}\left(T_{1}\right) \cap \operatorname{Dset}\left(T_{2}\right)=\emptyset$ in $E$.

Weak disjoint-access parallelism. Informally, weak DAP means that two transactions can concurrently contend on a common base object only if their data sets are connected in the conflict graph, capturing data-set overlaps among all concurrent transactions.

Let $G\left(T_{i}, T_{j}, E\right)$ be an undirected graph whose vertex set is $\bigcup_{T \in \tau_{E}\left(T_{i}, T_{j}\right)} \operatorname{Dset}(T)$ and there is an edge between t-objects $X$ and $Y$ iff there exists $T \in \tau_{E}\left(T_{i}, T_{j}\right)$ such that $\{X, Y\} \in D \operatorname{set}(T)$. We say that $T_{i}$ and $T_{j}$ are disjoint-access in $E$ if there is no path between a t-object in $\operatorname{Dset}\left(T_{i}\right)$ and a t-object in $\operatorname{Dset}\left(T_{j}\right)$ in $G\left(T_{i}, T_{j}, E\right)$. A TM implementation $M$ is weak disjoint-access parallel (weak DAP) if, for all executions $E$ of $M$, transactions $T_{i}$ and $T_{j}$ concurrently contend on the same base object in $E$ only if $T_{i}$ and $T_{j}$ are not disjoint-access in $E$ or there exists a t-object $X \in \operatorname{Dset}\left(T_{i}\right) \cap \operatorname{Dset}\left(T_{j}\right)$ [23, 107.

We now prove an auxiliary lemma, inspired by [23], concerning weak DAP TM implementations that will be useful in subsequent proofs. Intuitively, the lemma states that, two transactions that are disjointaccess and running one after the other in an execution of a weak DAP TM cannot contend on the same base object.

Lemma 2.10. Let $M$ be any weak DAP TM implementation. Let $\alpha \cdot \rho_{1} \cdot \rho_{2}$ be any execution of $M$ where $\rho_{1}$ (resp., $\rho_{2}$ ) is the step contention-free execution fragment of transaction $T_{1} \notin$ txns $(\alpha)$ (resp., $T_{2} \notin \operatorname{txns}(\alpha)$ ) and transactions $T_{1}, T_{2}$ are disjoint-access in $\alpha \cdot \rho_{1} \cdot \rho_{2}$. Then, $T_{1}$ and $T_{2}$ do not contend on any base object in $\alpha \cdot \rho_{1} \cdot \rho_{2}$.

Proof. Suppose, by contradiction that $T_{1}$ and $T_{2}$ contend on the same base object in $\alpha \cdot \rho_{1} \cdot \rho_{2}$.

If in $\rho_{1}, T_{1}$ performs a nontrivial event on a base object on which they contend, let $e_{1}$ be the last event in $\rho_{1}$ in which $T_{1}$ performs such an event to some base object $b$ and $e_{2}$, the first event in $\rho_{2}$ that accesses $b$. Otherwise, $T_{1}$ only performs trivial events in $\rho_{1}$ to base objects on which it contends with $T_{2}$ in $\alpha \cdot \rho_{1} \cdot \rho_{2}$ : let $e_{2}$ be the first event in $\rho_{2}$ in which $\rho_{2}$ performs a nontrivial event to some base object $b$ on which they contend and $e_{1}$, the last event of $\rho_{1}$ in $T_{1}$ that accesses $b$.

Let $\rho_{1}^{\prime}$ (and resp. $\rho_{2}^{\prime}$ ) be the longest prefix of $\rho_{1}$ (and resp. $\rho_{2}$ ) that does not include $e_{1}$ (and resp. $e_{2}$ ). Since before accessing $b$, the execution is step contention-free for $T_{1}, \alpha \cdot \rho_{1}^{\prime} \cdot \rho_{2}^{\prime}$ is an execution of $M$. By construction, $T_{1}$ and $T_{2}$ are disjoint-access in $\alpha \cdot \rho_{1}^{\prime} \cdot \rho_{2}^{\prime}$ and $\alpha \cdot \rho_{1} \cdot \rho_{2}^{\prime}$ is indistinguishable to $T_{2}$ from $\alpha \cdot \rho_{1}^{\prime} \cdot \rho_{2}^{\prime}$. Hence, $T_{1}$ and $T_{2}$ are poised to apply contending events $e_{1}$ and $e_{2}$ on $b$ in the configuration after $\alpha \cdot \rho_{1}^{\prime} \cdot \rho_{2}^{\prime}-$ a contradiction since $T_{1}$ and $T_{2}$ cannot concurrently contend on the same base object.

We now show that weak DAP is a weaker property than RW DAP.

Proposition 2.3. Weak $D A P \ll R W D A P$. 
Proof. Clearly, every implementation that satisfies RW DAP also satisfies weak DAP since the conflict graph $\tilde{G}\left(T_{i}, T_{j}, E\right)$ (for RW DAP) is a subgraph of $G\left(T_{i}, T_{j}, E\right)$ (for weak DAP).

However, the converse is not true (Algorithm 5.2 describes a TM implementation that satisfies weak DAP, but not RW DAP). Consider the following execution $E$ of a weak DAP TM implementaton $M$ that begins with the t-incomplete execution of a transaction $T_{0}$ that reads $X$ and writes to $Y$, followed by the step contention-free executions of two transactions $T_{1}$ and $T_{2}$ which write to $X$ and read $Y$ respectively. Transactions $T_{1}$ and $T_{2}$ may contend on a base object since there is a path between $X$ and $Y$ in $G\left(T_{1}, T_{2}, E\right)$. However, a RW DAP TM implementation would preclude transactions $T_{1}$ and $T_{2}$ from contending on the same base object: there is no edge between t-objects $X$ and $Y$ in the corresponding conflict graph $\tilde{G}\left(T_{1}, T_{2}, E\right)$ because $X$ and $Y$ are not contained in the write set of $T_{0}$.

Thus, the above propositions imply:

Corollary 2.11. Weak DAP $\ll W D A P \ll$ Strict DAP Strict data-partitioning.

\subsection{TM complexity metrics}

We now present an overview of some of the TM complexity metrics we consider in the thesis.

Step complexity. The step complexity metric, is the total number of events that a process performs on the shared memory, in the worst case, in order to complete its operation on the implementation.

RAW/AWAR patterns. Attiya et al. identified two common expensive synchronization patterns that frequently arise in the design of concurrent algorithms: read-after-write $(R A W)$ or atomic write-afterread $(A W A R)$ [16, 100] and showed that it is impossible to derive RAW/AWAR-free implementations of a wide class of data types that include sets, queues and deadlock-free mutual exclusion.

Note the shared memory model in the thesis makes the assumption that CPU events are performed atomically: every "read" of a base object returns the value of "latest write" to the base object. In practice however, the CPU architecture's memory model [3] that specifies the outcome of CPU instructions is relaxed without enforcing a strict order among the shared memory instructions. Intuitively, RAW (read-after-write) or AWAR (atomic-write-after-read) patterns [16] capture the amount of "expensive synchronization", i.e., the number of costly memory barriers or conditional primitives [3] incurred by the implementation in relaxed CPU architectures. The metric appears to be more practically relevant than simply counting the number of steps performed by a process, as it accounts for expensive cachecoherence operations or instructions like compare-and-swap. Detailed coverage on memory fences and the RAW/AWAR metric can be found in [100].

Definition 2.16 (Read-after-write metric). A RAW (read-after-write) pattern performed by a transaction $T_{k}$ in an execution $\pi$ is a pair of its events $e$ and $e^{\prime}$, such that: (1) $e$ is a write to a base object $b$ by $T_{k}$, (2) $e^{\prime}$ is a subsequent read of a base object $b^{\prime} \neq b$ by $T_{k}$, and (3) no event on by $T_{k}$ takes place between $e$ and $e^{\prime}$.

In the thesis, we are concerned only with non-overlapping RAWs, i.e., the read performed by one RAW precedes the write performed by the other RAW.

Definition 2.17 (Atomic write-after-read metric). An AWAR (atomic-write-after-read) pattern e in an execution $\pi \cdot e$ is a nontrivial rmw event on an object $b$ which atomically returns the value of $b$ (resulting after $\pi$ ) and updates $b$ with a new value.

For example, consider the execution $\pi \cdot e$ where $e$ is the application of a compare-and-swap rmw primitive that returns true.

Stall complexity. Intuitively, the stall metric captures the fact that the time a process might have to spend before it applies a primitive on a base object can be proportional to the number of processes that try to update the object concurrently. 
Let $M$ be any TM implementation. Let $e$ be an event applied by process $p$ to a base object $b$ as it performs a transaction $T$ during an execution $E$ of $M$. Let $E=\alpha \cdot e_{1} \cdots e_{m} \cdot e \cdot \beta$ be an execution of $M$, where $\alpha$ and $\beta$ are execution fragments and $e_{1} \cdots e_{m}$ is a maximal sequence of $m \geq 1$ consecutive nontrivial events by distinct distinct processes other than $p$ that access $b$. Then, we say that $T$ incurs $m$ memory stalls in $E$ on account of $e$. The number of memory stalls incurred by $T$ in $E$ is the sum of memory stalls incurred by all events of $T$ in $E$ [15, 45].

In the thesis, we adopt the following definition of a $k$-stall execution from [15, 45].

Definition 2.18. An execution $\alpha \cdot \sigma_{1} \cdots \sigma_{i}$ is a $k$-stall execution for t-operation op executed by process $p$ if

- $\alpha$ is p-free,

- there are distinct base objects $b_{1}, \ldots, b_{i}$ and disjoint sets of processes $S_{1}, \ldots, S_{i}$ whose union does not include $p$ and has cardinality $k$ such that, for $j=1, \ldots i$,

- each process in $S_{j}$ has an enabled nontrivial event about to access base object $b_{j}$ after $\alpha$, and

- in $\sigma_{j}, p$ applies events by itself until it is the first about to apply an event to $b_{j}$, then each of the processes in $S_{j}$ applies an event that accesses $b_{j}$, and finally, $p$ applies an event that accesses $b_{j}$,

- $p$ invokes exactly one t-operation op in the execution fragment $\sigma_{1} \cdots \sigma_{i}$

- $\sigma_{1} \cdots \sigma_{i}$ contains no events of processes not in $\left(\{p\} \cup S_{1} \cup \cdots \cup S_{i}\right)$

- in every $\left(\{p\} \cup S_{1} \cup \cdots \cup S_{i}\right)$-free execution fragment that extends $\alpha$, no process applies a nontrivial event to any base object accessed in $\sigma_{1} \cdots \sigma_{i}$.

Observe that in a $k$-stall execution $E$ for t-operation $o p$, the number of memory stalls incurred by op in $E$ is $k$

The following lemma will be of use in our proofs.

Lemma 2.12. Let $\alpha \cdot \sigma_{1} \cdots \sigma_{i}$ be a k-stall execution for t-operation op executed by process $p$. Then, $\alpha \cdot \sigma_{1} \cdots \sigma_{i}$ is indistinguishable to $p$ from a step contention-free execution [15].

Remote memory references(RMR) [21]. Modern shared memory CPU architectures employ a memory hierarchy [71: a hierarchy of memory devices with different capacities and costs. Some of the memory is local to a given process while the rest of the memory is remote. Accesses to memory locations (i.e. base objects) that are remote to a given process are often orders of magnitude slower than a local access of the base object. Thus, the performance of concurrent implementations in the shared memory model may depend on the number of remote memory references made to base objects [13].

In the cache-coherent (CC) shared memory, each process maintains local copies of shared base objects inside its cache, whose consistency is ensured by a coherence protocol. Informally, we say that an access to a base object $b$ is remote to a process $p$ and causes a remote memory reference ( $R M R$ ) if $p$ 's cache contains a cached copy of the object that is out of date or invalidated; otherwise the access is local.

In the write-through $(C C)$ protocol, to read a base object $b$, process $p$ must have a cached copy of $b$ that has not been invalidated since its previous read. Otherwise, $p$ incurs a RMR. To write to $b, p$ causes a RMR that invalidates all cached copies of $b$ and writes to the main memory.

In the write-back $(C C)$ protocol, $p$ reads a base object $b$ without causing a RMR if it holds a cached copy of $b$ in shared or exclusive mode; otherwise the access of $b$ causes a RMR that (1) invalidates all copies of $b$ held in exclusive mode, and writing $b$ back to the main memory, (2) creates a cached copy of $b$ in shared mode. Process $p$ can write to $b$ without causing a RMR if it holds a copy of $b$ in exclusive mode; otherwise $p$ causes a RMR that invalidates all cached copies of $b$ and creates a cached copy of $b$ in exclusive mode.

In the distributed shared memory (DSM), each base object is forever assigned to a single process and it is remote to the others. Any access of a remote register causes a RMR. 


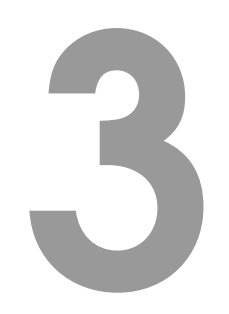

\section{Safety for transactional memory}

Arthur: If I asked you where the hell we were, would I regret it?

Ford: We're safe.

Arthur: Oh good.

Ford: We're in a small galley cabin in one of the spaceships of the Vogon Constructor Fleet.

Arthur: Ah, this is obviously some strange use of the word safe that I wasn't previously aware of.

Douglas Adams-The Hitchhiker's Guide to the Galaxy

\subsection{Overview}

In the context of Transactional memory, intermediate states witnessed by the read operations of an incomplete transaction may affect the user application through the outcome of its read operations. If the intermediate state is not consistent with any sequential execution, the application may experience a fatal irrecoverable error or enter an infinite loop. Thus, it is important that each transaction, including aborted ones observes a consistent state so that the implementation does not export any pathological executions.

A state should be considered consistent if it could result from a serial application of transactions observed in the current execution. In this sense, every transaction should witness a state that could have been observed in some execution of the sequential code put by the programmer within the transactions. Additionally, a consistent state should not depend on a transaction that has not started committing yet (referred to as deferred-update semantics). This restriction appears desirable, since the ongoing transaction may still abort (explicitly by the user or because of consistency reasons) and, thus, render the read inconsistent. Further, the set of histories specified by the consistency criterion must constitute a safety property, as defined by Owicki and Lamport [105], Alpern and Schneider [10] and refined by Lynch [97: it must be non-empty, prefix-closed and limit-closed. 
In this chapter, we define the notion of deferred-update semantics formally, which we then apply to a spectrum of TM consistency criteria. Additionally, we verify if the resulting TM consistency criterion is a safety property, as defined by Lynch [97.

We begin by considering the popular criterion of opacity 62, which was the first TM consistency criterion that was proposed to grasp this semantics formally. Opacity requires the states observed by all transactions, included uncommitted ones, to be consistent with a global serialization, i.e., a serial execution constituted by committed transactions. Moreover, the serialization should respect the real-time order: a transaction that completed before (in real time) another transaction started should appear first in the serialization.

By definition, opacity reduces correctness of a history to correctness of all its prefixes, and thus is prefix-closed and limit-closed by definition. Thus, to verify that a history is opaque, one needs to verify that each of its prefixes is consistent with some global serialization. To simplify verification and explicitly introduce deferred-update semantics into a TM correctness criterion, we specify a general criterion of du-opacity [17, which requires the global serial execution to respect the deferred-update property. Informally, a du-opaque history must be indistinguishable from a totally-ordered history, with respect to which no transaction reads from a transaction that has not started committing.

We show that du-opacity is prefix-closed, that is, every prefix of a du-opaque history is also du-opaque. We then show that extending opacity (and du-opacity) to infinite histories in a non-trivial way (i.e., requiring that even infinite histories should have proper serializations), does not result in a limit-closed property. However, under certain restrictions, we show that du-opacity is limit-closed. In particular, assuming that in an infinite history, every transaction completes each of the operations it invoked, the limit of any sequence of ever extending du-opaque histories is also du-opaque. Therefore, under this assumption, du-opacity is a safety property [10, 97, 105], and to prove that a TM implementation that complies with the assumption is du-opaque, it suffices to prove that all its finite histories are du-opaque.

One may notice that the intended safety semantics does not require that all transactions observe the same serial execution. Intuitively, to avoid pathological executions, we only need that every transaction witnesses some consistent state, while the views of different aborted and incomplete transactions do not have to be consistent with the same serial execution. As long as committed transactions constitute a serial execution and every transaction witnesses a consistent state, the execution can be considered "safe": no run-time error that cannot occur in a serial execution can happen. Several definitions like virtualworld consistency (VWC) [83] and Transactional Memory Specification 1 (TMS1) [2] have adopted this approach. We introduce "deferred-update" versions of these properties and discuss how the resulting properties relate to du-opacity.

Finally, we also study the consistency criterion Transactional Memory Specification 2 (TMS2) [42, 95], which was proposed as a restriction of opacity and verify if it is a safety property.

Roadmap of Chapter 3. In Section 3.2 of this chapter, we formally define safety properties. In Section 3.3 we introduce the notion of deferred-update semantics and apply it to the correctness criterions of opacity and strict serializability in Sections 3.4 and 3.5 respectively. Section 3.6 studies two relaxations of opacity: VWC and TMS1 and a restriction of opacity, TMS2. Section 3.7 summarizes the relations between the TM correctness properties proposed in the thesis and presents our concluding remarks.

\subsection{Safety properties}

A property $\mathcal{P}$ is a set of (transactional) histories. Intuitively, a safety property says that "no bad thing ever happens".

Definition 3.1 (Lynch [97]). A property $\mathcal{P}$ is a safety property if it satisfies the following two conditions:

Prefix-closure: For every history $H \in \mathcal{P}$, every prefix $H^{\prime}$ of $H$ (i.e., every prefix of the sequence of the events in $H$ ) is also in $\mathcal{P}$. 
Limit-closure: For every infinite sequence of finite histories $H^{0}, H^{1}, \ldots$ such that for every $i, H^{i} \in \mathcal{P}$ and $H^{i}$ is a prefix of $H^{i+1}$, the limit of the sequence is also in $\mathcal{P}$.

Notice that the set of histories produced by a TM implementation $M$ is, by construction, prefix-closed. Therefore, every infinite history of $M$ is the limit of an infinite sequence of ever-extending finite histories of $M$. Thus, to prove that $M$ satisfies a safety property $P$, it is enough to show that all finite histories of $M$ are in $P$. Indeed, limit-closure of $P$ then implies that every infinite history of $M$ is also in $P$.

\subsection{Opacity and deferred-update(DU) semantics}

In this section, we formalize the notion of deferred-update semantics and apply to the TM correctness condition of opacity 62$]$.

Definition 3.2 (Guerraoui and Kapalka [62]). A finite history $H$ is final-state opaque if there is a legal t-complete t-sequential history $S$, such that

1. for any two transactions $T_{k}, T_{m} \in \operatorname{txns}(H)$, if $T_{k} \prec_{H}^{R T} T_{m}$, then $T_{k}<_{S} T_{m}$, and

2. $S$ is equivalent to a completion of $H$.

We say that $S$ is a final-state serialization of $H$.

Final-state opacity is not prefix-closed. Figure 3.1 depicts a t-complete sequential history $H$ that is final-state opaque, with $T_{1} \cdot T_{2}$ being a legal t-complete t-sequential history equivalent to $H$. Let $H^{\prime}=$ write $_{1}(X, 1)$, read $d_{2}(X)$ be a prefix of $H$ in which $T_{1}$ and $T_{2}$ are t-incomplete. Transaction $T_{i}(i=1,2)$ is completed by inserting $\operatorname{try} C_{i} \cdot A_{i}$ immediately after the last event of $T_{i}$ in $H$. Observe that neither $T_{1} \cdot T_{2}$ nor $T_{2} \cdot T_{1}$ allow us to derive a serialization of $H^{\prime}$ (we assume that the initial value of $X$ is 0 ).

A restriction of final-state opacity, which we refer to as opacity [62] explicitly filters out histories that are not prefix-closed.

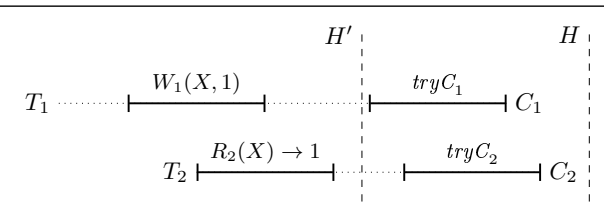

Figure 3.1: History $H$ is final-state opaque, while its prefix $H^{\prime}$ is not final-state opaque.

Definition 3.3 (Guerraoui and Kapalka [62]). A history $H$ is opaque if and only if every finite prefix $H^{\prime}$ of $H$ (including $H$ itself if it is finite) is final-state opaque.

It can be easily seen that opacity is prefix- and limit-closed, and, thus, it is a safety property.

We now give a formal definition of opacity with deferred-update semantics. Then we show that the property is prefix-closed and, under certain liveness restrictions, limit-closed.

Let $H$ be any history and let $S$ be a legal t-complete t-sequential history that is equivalent to some completion of $H$. Let $<_{S}$ be the total order on transactions in $S$.

Definition 3.4 (Local serialization). For any read red $_{k}(X)$ that does not return $A_{k}$, let $S^{k, X}$ be the prefix of $S$ up to the response of $\operatorname{read}_{k}(X)$ and $H^{k, X}$ be the prefix of $H$ up to the response of $\operatorname{read}_{k}(X)$. $S_{H}^{k, X}$, the local serialization of read $_{k}(X)$ with respect to $H$ and $S$, is the subsequence of $S^{k, X}$ derived by removing from $S^{k, X}$ the events of all transactions $T_{m} \in \operatorname{txns}(H) \backslash\left\{T_{k}\right\}$ such that $H^{k, X}$ does not contain an invocation of $\operatorname{try} C_{m}()$. 
We are now ready to present our correctness condition, du-opacity.

Definition 3.5 (Du-opacity). A history $H$ is du-opaque if there is a legal $t$-complete $t$-sequential history S such that

1. there is a completion of $H$ that is equivalent to $S$, and

2. for every pair of transactions $T_{k}, T_{m} \in \operatorname{txns}(H)$, if $T_{k} \prec_{H}^{R T} T_{m}$, then $T_{k}<_{S} T_{m}$, i.e., S respects the real-time ordering of transactions in $H$, and

3. each $\operatorname{read}_{k}(X)$ in $S$ that does not return $A_{k}$ is legal in $S_{H}^{k, X}$.

We then say that $S$ is a (du-opaque) serialization of $H$.

Informally, a history $H$ is du-opaque if there is a legal t-sequential history $S$ that is equivalent to $H$, respects the real-time ordering of transactions in $H$ and every t-read is legal in its local serialization with respect to $H$ and $S$. The third condition reflects the implementation's deferred-update semantics, i.e., the legality of a t-read in a serialization does not depend on transactions that start committing after the response of the t-read.

For any du-opaque serialization $S, \operatorname{seq}(S)$ denotes the sequence of transactions in $S$ and $\operatorname{seq}(S)[k]$ denotes the $k^{\text {th }}$ transaction in this sequence.

\subsection{On the safety of du-opacity}

In this section, we examine the safety properties of du-opacity, i.e., whether it is prefix-closed and limit-closed.

\subsubsection{Du-opacity is prefix-closed}

Lemma 3.1. Let $H$ be a du-opaque history and let $S$ be a serialization of $H$. For any $i \in \mathbb{N}$, there is a serialization $S^{i}$ of $H^{i}$ (the prefix of $H$ consisting of the first $i$ events), such that $\operatorname{seq}\left(S^{i}\right)$ is a subsequence of $\operatorname{seq}(S)$.

Proof. Given $H, S$ and $H^{i}$, we construct a t-complete t-sequential history $S^{i}$ as follows:

- for every transaction $T_{k}$ that is t-complete in $H^{i}, S^{i}|k=S| k$.

- for every transaction $T_{k}$ that is complete but not t-complete in $H^{i}, S^{i} \mid k$ consists of the sequence of events in $H^{i} \mid k$, immediately followed by $\operatorname{try} C_{k}() \cdot A_{k}$.

- for every transaction $T_{k}$ with an incomplete t-operation, op $_{k}=\operatorname{read}_{k} \vee$ write $_{k} \vee \operatorname{try} A_{k}()$ in $H^{i}, S^{i} \mid k$ is the sequence of events in $S \mid k$ up to the invocation of $o p_{k}$, immediately followed by $A_{k}$.

- for every transaction $T_{k} \in \operatorname{txns}\left(H^{i}\right)$ with an incomplete t-operation, $o p_{k}=\operatorname{try} C_{k}(), S^{i}|k=S| k$.

By the above construction, $S^{i}$ is indeed a t-complete history and every transaction that appears in $S^{i}$ also appears in $S$. We order transactions in $S^{i}$ so that $\operatorname{seq}\left(S^{i}\right)$ is a subsequence of $\operatorname{seq}(S)$.

Note that $S^{i}$ is derived from events contained in some completion $\bar{H}$ of $H$ that is equivalent to $S$ and some other events to derive a completion of $S^{i}$. Since $S^{i}$ contains events from every complete t-operation in $H^{i}$ and other events included satisfy Definition 2.1, there is a completion of $H^{i}$ that is equivalent to $S^{i}$.

We now argue that $S^{i}$ is a serialization of $H^{i}$. First we observe that $S^{i}$ respects the real-time order of $H^{i}$. Indeed, if $T_{j} \prec_{H^{i}}^{R T} T_{k}$, then $T_{j} \prec_{H}^{R T} T_{k}$ and $T_{j}<_{S} T_{k}$. Since $\operatorname{seq}\left(S^{i}\right)$ is a subsequence of $\operatorname{seq}(S)$, we have $T_{j}<_{S^{i}} T_{k}$. 


$$
\begin{aligned}
& T_{1} \longmapsto W_{1}(X, 1) \\
& T_{2} \longmapsto R_{2}(X) \rightarrow 1 \\
& T_{3} \stackrel{R_{3}(X) \rightarrow 0}{\longmapsto}
\end{aligned}
$$

Figure 3.2: An infinite history in which $\operatorname{try} C_{1}$ is incomplete and any two transactions are concurrent. Each finite prefix of the history is du-opaque, but the infinite limit of the ever-extending sequence is not du-opaque.

To show that $S^{i}$ is legal, suppose, by way of contradiction, that there is some read $_{k}(X)$ that returns $v \neq A_{k}$ in $H^{i}$ such that $v$ is not the latest written value of $X$ in $S^{i}$. If $T_{k}$ contains a write $e_{k}\left(X, v^{\prime}\right)$ preceding $\operatorname{read}_{k}(X)$ such that $v \neq v^{\prime}$ and $v$ is not the latest written value for $\operatorname{read}_{k}(X)$ in $S^{i}$, it is also not the latest written value for $\operatorname{read}_{k}(X)$ in $S$, which is a contradiction. Thus, the only case to consider is when $\operatorname{read}_{k}(X)$ should return a value written by another transaction.

Since $S$ is a serialization of $H$, there is a committed transaction $T_{m}$ that performs the last write $e_{m}(X, v)$ that precedes $\operatorname{read}_{k}(X)$ in $T_{k}$ in $S$. Moreover, since $\operatorname{read}_{k}(X)$ is legal in the local serialization of $\operatorname{read}_{k}(X)$ in $H$ with respect to $S$, the prefix of $H$ up to the response of $\operatorname{read}_{k}(X)$ must contain an invocation of $\operatorname{try} C_{m}()$. Thus, $\operatorname{read}_{k}(X) \nprec_{H}^{R T} \operatorname{try} C_{m}()$ and $T_{m} \in \operatorname{txns}\left(H^{i}\right)$. By construction of $S^{i}, T_{m} \in \operatorname{txns}\left(S^{i}\right)$ and $T_{m}$ is committed in $S^{i}$.

We have assumed, towards a contradiction, that $v$ is not the latest written value for $\operatorname{read}_{k}(X)$ in $S^{i}$. Hence, there is a committed transaction $T_{j}$ that performs write $j\left(X, v^{\prime}\right) ; v^{\prime} \neq v$ in $S^{i}$ such that $T_{m}<_{S^{i}}$ $T_{j}<_{S^{i}} T_{k}$. But this is not possible since $\operatorname{seq}\left(S^{i}\right)$ is a subsequence of $\operatorname{seq}(S)$.

Thus, $S^{i}$ is a legal t-complete t-sequential history equivalent to some completion of $H^{i}$. Now, by the construction of $S^{i}$, for every $\operatorname{read}_{k}(X)$ that does not return $A_{k}$ in $S^{i}$, we have $S_{H^{i}}^{i^{k, X}}=S_{H}^{k, X}$. Indeed, the transactions that appear before $T_{k}$ in $S_{H^{i}}^{i, X}$ are those with a $\operatorname{try} C$ event before the response of $\operatorname{read}_{k}(X)$ in $H$ and are committed in $S$. Since $\operatorname{seq}\left(S^{i}\right)$ is a subsequence of $\operatorname{seq}(S)$, we have $S_{H^{i}}^{k, X}=S_{H}^{k, X}$. Thus, $\operatorname{read}_{k}(X)$ is legal in $S_{H^{i}}^{i, X}$.

Lemma 3.1 implies that every prefix of a du-opaque history has a du-opaque serialization and thus:

Corollary 3.2. Du-opacity is a prefix-closed property.

\subsubsection{The limit of du-opaque histories}

We observe, however, that du-opacity is, in general, not limit-closed. We present an infinite history that is not du-opaque, but each of its prefixes is.

Proposition 3.1. Du-opacity is not a limit-closed property.

Proof. Let $H^{j}$ denote a finite prefix of $H$ of length $j$. Consider an infinite history $H$ that is the limit of the histories $H^{j}$ defined as follows (see Figure 3.2):

- Transaction $T_{1}$ performs a write $_{1}(X, 1)$ and then invokes try $C_{1}()$ that is incomplete in $H$.

- Transaction $T_{2}$ performs a $\operatorname{read}_{2}(X)$ that overlaps with $\operatorname{try} C_{1}()$ and returns 1.

- There are infinitely many transactions $T_{i}, i \geq 3$, each of which performing a single $\operatorname{read}_{i}(X)$ that returns 0 such that each $T_{i}$ overlaps with both $T_{1}$ and $T_{2}$.

We now prove that, for all $j \in \mathbb{N}, H^{j}$ is a du-opaque history. Clearly, $H^{0}$ and $H^{1}$ are du-opaque histories. For all $j>1$, we first derive a completion of $H^{j}$ as follows: 
1. $\operatorname{try} C_{1}\left(\right.$ ) (if it is contained in $H^{j}$ ) is completed by inserting $C_{1}$ immediately after its invocation,

2. for all $i \geq 2$, any incomplete $\operatorname{read}_{i}(X)$ that is contained in $H^{j}$ is completed by inserting $A_{i}$ and $\operatorname{try} C_{i} \cdot A_{i}$ immediately after its invocation, and

3. for all $i \geq 2$ and every complete $\operatorname{read}_{j}(X)$ that is contained in $H^{j}$, we include $\operatorname{try} C_{i} \cdot A_{i}$ immediately after the response of this $\operatorname{read}_{j}(X)$.

We can now derive a t-complete t-sequential history $S^{j}$ equivalent to the above derived completion of $H^{j}$ from the sequence of transactions $T_{3}, \ldots, T_{i}, T_{1}, T_{2}$ (depending on which of these transactions participate in $H^{j}$ ), where $i \geq 3$. It is easy to observe that $S^{j}$ so derived is indeed a serialization of $H^{j}$.

However, there is no serialization of $H$. Suppose that such a serialization $S$ exists. Since every transaction that participates in $H$ must participate in $S$, there exists $n \in \mathbb{N}$ such that $\operatorname{seq}(S)[n]=T_{1}$. Consider the transaction at index $n+1$, say $T_{i}$ in $\operatorname{seq}(S)$. But for any $i \geq 3, T_{i}$ must precede $T_{1}$ in any serialization (by legality), which is a contradiction.

Notice that all finite prefixes of the infinite history depicted in Figure 3.2 are also opaque. Thus, if we extend the definition of opacity to cover infinite histories in a non-trivial way, i. e., by explicitly defining opaque serializations for infinite histories, we can reformulate Proposition 3.1 for opacity.

\subsubsection{Du-opacity is limit-closed for complete histories}

We show now that du-opacity is limit-closed if the only infinite histories we consider are those in which every transaction eventually completes (but not necessarily t-completes).

We first prove an auxiliary lemma on du-opaque serializations. For a transaction $T \in \operatorname{txns}(H)$, the live set of $T$ in $H$, denoted $\operatorname{Lset}_{H}(T)$ ( $T$ included), is defined as follows: every transaction $T^{\prime} \in \operatorname{txns}(H)$ such that neither the last event of $T^{\prime}$ precedes the first event of $T$ in $H$ nor the last event of $T$ precedes the first event of $T^{\prime}$ in $H$ is contained in $\operatorname{Lset}_{H}(T)$. We say that transaction $T^{\prime} \in$ txns $(H)$ succeeds the live set of $T$ and we write $T \prec_{H}^{L S} T^{\prime}$ if in $H$, for all $T^{\prime \prime} \in \operatorname{Lset}_{H}(T), T^{\prime \prime}$ is complete and the last event of $T^{\prime \prime}$ precedes the first event of $T^{\prime}$.

Lemma 3.3. Let $H$ be a finite du-opaque history and assume $T_{k} \in \operatorname{txns}(H)$ is a complete transaction in $H$, such that every transaction in Lset $_{H}\left(T_{k}\right)$ is complete in $H$. Then there is a serialization $S$ of $H$, such that for all $T_{k}, T_{m} \in \operatorname{txns}(H)$, if $T_{k} \prec_{H}^{L S} T_{m}$, then $T_{k}<_{S} T_{m}$.

Proof. Since $H$ is du-opaque, there is a serialization $\tilde{S}$ of $H$.

Let $S$ be a t-complete t-sequential history such that $\operatorname{txns}(\tilde{S})=\operatorname{txns}(S)$, and $\forall T_{i} \in \operatorname{txns}(\tilde{S}): S|i=\tilde{S}| i$. We now perform the following procedure iteratively to derive $\operatorname{seq}(S)$ from $\operatorname{seq}(\tilde{S})$. Initially $\operatorname{seq}(S)=\operatorname{seq}(\tilde{S})$. For each $T_{k} \in \operatorname{txns}(H)$, let $T_{\ell} \in \operatorname{txns}(H)$ denote the earliest transaction in $\tilde{S}$ such that $T_{k} \prec_{H}^{L S} T_{\ell}$. If $T_{\ell}<_{\tilde{S}} T_{k}$ (implying $T_{k}$ is not t-complete), then move $T_{k}$ to immediately precede $T_{\ell}$ in $\operatorname{seq}(S)$.

By construction, $S$ is equivalent to $\tilde{S}$ and for all $T_{k}, T_{m} \in \operatorname{txns}(H) ; T_{k} \prec_{H}^{L S} T_{m}, T_{k}<_{S} T_{m}$ We claim that $S$ is a serialization of $H$. Observe that any two transactions that are complete in $H$, but not t-complete are not related by real-time order in $H$. By construction of $S$, for any transaction $T_{k} \in \operatorname{txns}(H)$, the set of transactions that precede $T_{k}$ in $\tilde{S}$, but succeed $T_{k}$ in $S$ are not related to $T_{k}$ by real-time order. Since $\tilde{S}$ respects the real-time order in $H$, this holds also for $S$.

We now show that $S$ is legal. Consider any $\operatorname{read}_{k}(X)$ performed by some transaction $T_{k}$ that returns $v \in V$ in $S$ and let $T_{\ell} \in \operatorname{txns}(H)$ be the earliest transaction in $\widetilde{S}$ such that $T_{k} \prec H T_{\ell}$. Suppose, by contradiction, that $\operatorname{read}_{k}(X)$ is not legal in $S$. Thus, there is a committed transaction $T_{m}$ that performs write $_{m}(X, v)$ in $\tilde{S}$ such that $T_{m}=T_{\ell}$ or $T_{\ell}<_{\tilde{S}} T_{m}<_{\tilde{S}} T_{k}$. Note that, by our assumption, $\operatorname{read}_{k}(X) \prec_{H}^{R T} \operatorname{try} C_{\ell}()$. Since $\operatorname{read}_{k}(X)$ must be legal in its local serialization with respect to $H$ and $\tilde{S}$, $\operatorname{read}_{k}(X) \nprec_{H}^{R T} \operatorname{try} C_{m}()$. Thus, $T_{m} \in \operatorname{Lset}_{H}\left(T_{k}\right)$. Therefore $T_{m} \neq T_{\ell}$. Moreover, $T_{m}$ is complete, and since it commits in $\widetilde{S}$, it is also t-complete in $H$ and the last event of $T_{m}$ precedes the first event of $T_{\ell}$ in $H$, i.e., $T_{m} \prec_{H}^{R T} T_{\ell}$. Hence, $T_{\ell}$ cannot precede $T_{m}$ in $\tilde{S}-$ a contradiction. 
Observe also that since $T_{k}$ is complete in $H$ but not t-complete, $H$ does not contain an invocation of $\operatorname{try} C_{k}()$. Thus, the legality of any other transaction is unaffected by moving $T_{k}$ to precede $T_{\ell}$ in $S$. Thus, $S$ is a legal t-complete t-sequential history equivalent to some completion of $H$. The above arguments also prove that every t-read in $S$ is legal in its local serialization with respect to $H$ and $S$ and, thus, $S$ is a serialization of $H$.

The proof uses König's Path Lemma [85] formulated as follows. Let $G$ on a rooted directed graph and let $v_{0}$ be the root of $G$. We say that $v_{k}$, a vertex of $G$, is reachable from $v_{0}$, if there is a sequence of vertices $v_{0} \ldots, v_{k}$ such that for each $i$, there is an edge from $v_{i}$ to $v_{i+1}$. $G$ is connected if every vertex in $G$ is reachable from $v_{0} . G$ is finitely branching if every vertex in $G$ has a finite out-degree. $G$ is infinite if it has infinitely many vertices.

Lemma 3.4 (König's Path Lemma [85]). If $G$ is an infinite connected finitely branching rooted directed graph, then $G$ contains an infinite sequence of distinct vertices $v_{0}, v_{1}, \ldots$, such that $v_{0}$ is the root, and for every $i \geq 0$, there is an edge from $v_{i}$ to $v_{i+1}$.

Theorem 3.5. Under the restriction that in any infinite history $H$, every transaction $T_{k} \in \operatorname{txns}(H)$ is complete, du-opacity is a limit-closed property.

Proof. We want to show that the limit $H$ of an infinite sequence of finite ever-extending du-opaque histories is du-opaque. By Corollary 3.2, we can assume the sequence of du-opaque histories to be $H^{0}, H^{1}, \ldots H^{i}, H^{i+1}, \ldots$ such that for all $i \in \mathbb{N}, H^{i+1}$ is the one-event extension of $H^{i}$.

We construct a rooted directed graph $G_{H}$ as follows:

1. The root vertex of $G_{H}$ is $\left(H^{0}, S^{0}\right)$ where $S^{0}$ and $H^{0}$ contain the initial transaction $T_{0}$.

2. Each non-root vertex of $G_{H}$ is a tuple $\left(H^{i}, S^{i}\right)$, where $S^{i}$ is a du-opaque serialization of $H^{i}$ that satisfies the condition specified in Lemma 3.3 for all $T_{k}, T_{m} \in \operatorname{txns}(H) ; T_{k} \prec_{H^{i}}^{L S} T_{m}$ implies $T_{k}<_{S^{i}} T_{m}$. Note that there exist several possible serializations for any $H^{i}$. For succinctness, in the rest of this proof, when we refer to a specific $S^{i}$, it is understood to be associated with the prefix $H^{i}$ of $H$.

3. Let $\operatorname{cseq}_{i}\left(S^{j}\right), j \geq i$, denote the subsequence of $\operatorname{seq}\left(S^{j}\right)$ restricted to transactions whose last event in $H$ is a response event and it is contained in $H^{i}$. For every pair of vertices $v=\left(H^{i}, S^{i}\right)$ and $v^{\prime}=\left(H^{i+1}, S^{i+1}\right)$ in $G_{H}$, there is an edge from $v$ to $v^{\prime}$ if $\operatorname{cseq}_{i}\left(S^{i}\right)=\operatorname{cseq}_{i}\left(S^{i+1}\right)$.

The out-degree of a vertex $v=\left(H^{i}, S^{i}\right)$ in $G_{H}$ is defined by the number of possible serializations of $H^{i+1}$, bounded by the number of possible permutations of the set $\operatorname{txns}\left(S^{i+1}\right)$, implying that $G_{H}$ is finitely branching.

By Lemma 3.1. given any serialization $S^{i+1}$ of $H^{i+1}$, there is a serialization $S^{i}$ of $H^{i}$ such that $\operatorname{seq}\left(S^{i}\right)$ is a subsequence of $\operatorname{seq}\left(S^{i+1}\right)$. Indeed, the serialization $S^{i}$ of $H^{i}$ also respects the restriction specified in Lemma 3.3. Since $\operatorname{seq}\left(S^{i+1}\right)$ contains every complete transaction that takes its last step in $H$ in $H^{i}$, $\operatorname{cseq}_{i}\left(S^{i}\right)=\operatorname{cseq}_{i}\left(S^{i+1}\right)$. Therefore, for every vertex $\left(H^{i+1}, S^{i+1}\right)$, there is a vertex $\left(H^{i}, S^{i}\right)$ such that $\operatorname{cseq}_{i}\left(S^{i}\right)=\operatorname{cseq}_{i}\left(S^{i+1}\right)$. Thus, we can iteratively construct a path from $\left(H^{0}, S^{0}\right)$ to every vertex $\left(H^{i}, S^{i}\right)$ in $G_{H}$, implying that $G_{H}$ is connected.

We now apply König's Path Lemma (Lemma 3.4) to $G_{H}$. Since $G_{H}$ is an infinite connected finitely branching rooted directed graph, we can derive an infinite sequence of distinct vertices

$$
\mathcal{L}=\left(H^{0}, S^{0}\right),\left(H^{1}, S^{1}\right), \ldots,\left(H^{i}, S^{i}\right), \ldots
$$

such that $\operatorname{cseq}_{i}\left(S^{i}\right)=\operatorname{cseq}_{i}\left(S^{i+1}\right)$.

The rest of the proof explains how to use $\mathcal{L}$ to construct a serialization of $H$. We begin with the following claim concerning $\mathcal{L}$.

Claim 3.6. For any $j>i, \operatorname{cseq}_{i}\left(S^{i}\right)=\operatorname{cseq}_{i}\left(S^{j}\right)$. 
Proof. Recall that $\operatorname{cseq}_{i}\left(S^{i}\right)$ is a prefix of $\operatorname{cseq}_{i}\left(S^{i+1}\right)$, and $\operatorname{cseq}_{i+1}\left(S^{i+1}\right)$ is a prefix of $c s e q_{i+1}\left(S^{i+2}\right)$. Also, $\operatorname{cseq}_{i}\left(S^{i+1}\right)$ is a subsequence of $c s e q_{i+1}\left(S^{i+1}\right)$. Hence, $\operatorname{cseq}_{i}\left(S^{i}\right)$ is a subsequence of $c s e q_{i+1}\left(S^{i+2}\right)$. But, $\operatorname{cseq}_{i+1}\left(S^{i+2}\right)$ is a subsequence of $\operatorname{cseq}_{i+2}\left(S^{i+2}\right)$. Thus, $\operatorname{cseq}_{i}\left(S^{i}\right)$ is a subsequence of $c s e q_{i+2}\left(S^{i+2}\right)$. Inductively, for any $j>i, \operatorname{cseq}_{i}\left(S^{i}\right)$ is a subsequence of $\operatorname{cseq}_{j}\left(S^{j}\right)$. But $\operatorname{cseq}_{i}\left(S^{j}\right)$ is the subsequence of $\operatorname{cseq}_{j}\left(S^{j}\right)$ restricted to complete transactions in $H$ whose last step is in $H^{i}$. Thus, $\operatorname{cseq}_{i}\left(S^{i}\right)$ is indeed equal to $\operatorname{cseq}_{i}\left(S^{j}\right)$.

Let $f: \mathbb{N} \rightarrow \operatorname{txns}(H)$ be defined as follows: $f(1)=T_{0}$. For every integer $k>1$, let

$$
i_{k}=\min \left\{\ell \in \mathbb{N} \mid \forall j>\ell: \operatorname{cseq}_{\ell}\left(S^{\ell}\right)[k]=\operatorname{cseq}_{j}\left(S^{j}\right)[k]\right\}
$$

Then, $f(k)=\operatorname{cseq}_{i_{k}}\left(S^{i_{k}}\right)[k]$.

Claim 3.7. The function $f$ is total and bijective.

\section{Proof. (Totality and surjectivity)}

Since each transaction $T \in \operatorname{txns}(H)$ is complete in some prefix $H^{i}$ of $H$, for each $k \in \mathbb{N}$, there exists $i \in \mathbb{N}$ such that $\operatorname{cseq}_{i}\left(S^{i}\right)[k]=T$. By Claim 3.6. for any $j>i, \operatorname{cseq}_{i}\left(S^{i}\right)=\operatorname{cseq}_{i}\left(S^{j}\right)$. Since a transaction that is complete in $H^{i}$ w.r.t $H$ is also complete in $H^{j}$ w.r.t $H$, it follows that for every $j>i, \operatorname{cseq}_{j}\left(S^{j}\right)\left[k^{\prime}\right]=T$, with $k^{\prime} \geq k$. By construction of $G_{H}$ and the assumption that each transaction is complete in $H$, there exists $i \in \mathbb{N}$ such that each $T \in \operatorname{Lset}_{H^{i}}(T)$ is complete in $H$ and its last step is in $H^{i}$, and $T$ precedes in $S^{i}$ every transaction whose first event succeeds the last event of each $T^{\prime} \in L_{s e t} H^{i}(T)$ in $H^{i}$. Indeed, this implies that for each $k \in \mathbb{N}$, there exists $i \in \mathbb{N}$ such that $\operatorname{cseq}_{i}\left(S^{i}\right)[k]=T ; \forall j>i: \operatorname{cseq}_{j}\left(S^{j}\right)[k]=T$.

This shows that for every $T \in \operatorname{txns}(H)$, there are $i, k \in \mathbb{N} ; \operatorname{cseq}_{i}\left(S^{i}\right)[k]=T$, such that for every $j>i$, $\operatorname{cseq}_{j}\left(S^{j}\right)[k]=T$. Thus, for every $T \in \operatorname{txns}(H)$, there is $k$ such that $f(k)=T$.

(Injectivity)

If $f(k)$ and $f(m)$ are transactions at indices $k, m$ of the same $\operatorname{cseq}_{i}\left(S^{i}\right)$, then clearly $f(k)=f(m)$ implies $k=m$. Suppose $f(k)$ is the transaction at index $k$ in some $\operatorname{cseq}_{i}\left(S^{i}\right)$ and $f(m)$ is the transaction at index $m$ in some $\operatorname{cseq}_{\ell}\left(S^{\ell}\right)$. For every $\ell>i$ and $k<m$, if $\operatorname{cseq}_{i}\left(S^{i}\right)[k]=T$, then $\operatorname{cseq}_{\ell}\left(S^{\ell}\right)[m] \neq T$ since $\operatorname{cseq}_{i}\left(S^{i}\right)=\operatorname{cseq}_{i}\left(S^{\ell}\right)$. If $\ell>i$ and $k>m$, it follows from the definition that $f(k) \neq f(m)$. Similar arguments for the case when $\ell<i$ prove that if $f(k)=f(m)$, then $k=m$.

By Claim 3.7, $\mathcal{F}=f(1), f(2), \ldots, f(i), \ldots$ is an infinite sequence of transactions. Let $S$ be a t-complete t-sequential history such that $\operatorname{seq}(S)=\mathcal{F}$ and for each t-complete transaction $T_{k}$ in $H, S|k=H| k$; and for transaction that is complete, but not t-complete in $H, S \mid k$ consists of the sequence of events in $H \mid k$, immediately followed by $\operatorname{try} A_{k}() \cdot A_{k}$. Clearly, there is a completion of $H$ that is equivalent to $S$.

Let $\mathcal{F}^{i}$ be the prefix of $\mathcal{F}$ of length $i$, and $\widehat{S}^{i}$ be the prefix of $S$ such that $\operatorname{seq}\left(\widehat{S}^{i}\right)=\mathcal{F}^{i}$.

Claim 3.8. Let $\widehat{H}_{i}^{j}$ be a subsequence of $H^{j}$ reduced to transactions $T_{k} \in \operatorname{txns}\left(\widehat{S}^{i}\right)$ such that the last event of $T_{k}$ in $H$ is a response event and it is contained in $H^{j}$. Then, for every $i$, there is $j$ such that $\widehat{S}^{i}$ is a serialization of $\widehat{H}_{i}^{j}$.

Proof. Let $H^{j}$ be the shortest prefix of $H$ (from $\mathcal{L}$ ) such that for each $T \in \operatorname{txns}\left(\widehat{S}^{i}\right)$, if $\operatorname{seq}\left(S^{j}\right)[k]=T$, then for every $j^{\prime}>j, \operatorname{seq}\left(S^{j^{\prime}}\right)[k]=T$. From the construction of $\mathcal{F}$, such $j$ and $k$ exist. Also, we observe that $\operatorname{txns}\left(\widehat{S}^{i}\right) \subseteq \operatorname{txns}\left(S^{j}\right)$ and $\mathcal{F}^{i}$ is a subsequence of $\operatorname{seq}\left(S^{j}\right)$. Using arguments similar to the proof of Lemma 3.1 it follows that $\widehat{S}^{i}$ is indeed a serialization of $\widehat{H}_{i}^{j}$.

Since $H$ is complete, there is exactly one completion of $H$, where each transaction $T_{k}$ that is not tcomplete in $H$ is completed with $\operatorname{try} C_{k} \cdot A_{k}$ after its last event. By Claim 7.11, the limit t-sequential t-complete history is equivalent to this completion, is legal, respects the real-time order of $H$, and ensures that every read is legal in the corresponding local serialization. Thus, $S$ is a serialization of $H$. 


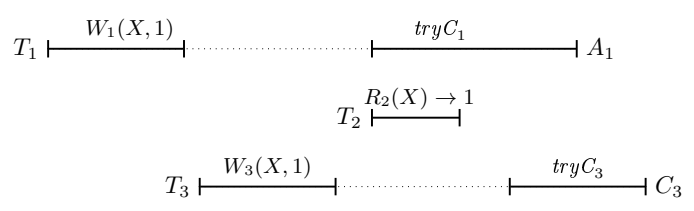

Figure 3.3: A history that is opaque, but not du-opaque.

Theorem 3.5 implies the following:

Corollary 3.9. Let $M$ be a TM implementation that ensures that in every infinite history $H$ of $M$, every transaction $T \in \operatorname{txns}(H)$ is complete in $H$. Then, $M$ is du-opaque if and only if every finite history of $M$ is du-opaque.

\subsubsection{Du-opacity vs. opacity}

We now compare our deferred-update requirement with the conventional TM correctness property of opacity 62 .

Theorem 3.10. Du-opacity $\varsubsetneqq$ Opacity.

Proof. We first claim that every finite du-opaque history is opaque. Let $H$ be a finite du-opaque history. By definition, there is a final-state serialization $S$ of $H$. Since du-opacity is a prefix-closed property, every prefix of $H$ is final-state opaque. Thus, $H$ is opaque.

Again, since every prefix of a du-opaque history is also du-opaque, by Definition 3.3 every infinite du-opaque history is also opaque.

To show that the inclusion is strict, we present an an opaque history that is not du-opaque. Consider the finite history $H$ depicted in Figure 3.3 transaction $T_{2}$ performs a $\operatorname{read}_{2}(X)$ that returns the value 1. Observe that $\operatorname{read}_{2}(X) \rightarrow 1$ is concurrent to $\operatorname{try} C_{1}$, but precedes $\operatorname{try} C_{3}$ in real-time order. Although try $C_{1}$ returns $A_{1}$ in $H$, the response of $\operatorname{read}_{2}(X)$ can be justified since $T_{3}$ concurrently writes 1 to $X$ and commits. Thus, read $_{2}(X) \rightarrow 1$ reads-from transaction $T_{2}$ in any serialization of $H$, but since $\operatorname{read}_{2}(X) \prec_{H}^{R T} \operatorname{try} C_{3}, H$ is not du-opaque even though each of its prefixes is final-state opaque.

We now formally prove that $H$ is opaque. We proceed by examining every prefix of $H$.

1. Each prefix up to the invocation of $\operatorname{read}_{2}(X)$ is trivially final-state opaque.

2. Consider the prefix, $H^{i}$ of $H$ where the $i^{t h}$ event is the response of $\operatorname{read}_{2}(X)$. Let $S^{i}$ be a tcomplete t-sequential history derived from the sequence $T_{1}, T_{2}$ by inserting $C_{1}$ immediately after the invocation of $\operatorname{try} C_{1}()$. It is easy to see that $S^{i}$ is a final-state serialization of $H^{i}$.

3. Consider the t-complete t-sequential history $S$ derived from the sequence $T_{1}, T_{3}, T_{2}$ in which each transaction is t-complete in $H$. Clearly, $S$ is a final-state serialization of $H$.

Since $H$ and every (proper) prefix of it are final-state opaque, $H$ is opaque.

Clearly, the required final-state serialization $S$ of $H$ is specified by $\operatorname{seq}(S)=T_{1}, T_{3}, T_{2}$ in which $T_{1}$ is aborted while $T_{3}$ is committed in $S$ (the position of $T_{1}$ in the serialization does not affect legality). Consider read $_{2}(X)$ in $S$; since $H^{2, X}$, the prefix of $H$ up to the response of read $_{2}(X)$ does not contain an invocation of $\operatorname{try} C_{3}()$, the local serialization of $\operatorname{read}_{2}(X)$ with respect to $H$ and $S, S_{H}^{2, X}$ is $T_{1} \cdot \operatorname{read}_{2}(X)$. But $\operatorname{read}_{2}(X)$ is not legal in $S_{H}^{2, X}$, which is a contradiction. Thus, $H$ is not du-opaque.

The unique-write case We now show that du-opacity is equivalent to opacity assuming that no two transactions write identical values to the same t-object ("unique-write" assumption).

Let Opacity uw $_{\subseteq}$ Opacity, be a property defined as follows: 


$$
\begin{aligned}
& T_{3} \longmapsto W_{3}(X, 1), W_{3}(Y, 1), C_{3}
\end{aligned}
$$

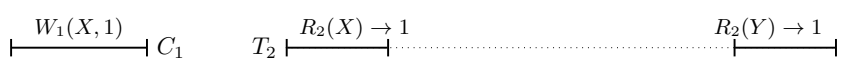

Figure 3.4: A sequential du-opaque history, which is not opaque by the definition of [57].

1. an infinite opaque history $H \in$ Opacity $_{u w}$ if and only if every transaction $T \in \operatorname{txns}(H)$ is complete in $H$, and

2. an opaque history $H \in$ Opacity $_{u w}$ if and only if for every pair of write operations write $_{k}(X, v)$ and write $_{m}\left(X, v^{\prime}\right), v \neq v^{\prime}$.

Theorem 3.11. Opacity Ow $_{1}=d u$-opacity.

Proof. We show first that every finite history $H \in$ Opacity $_{u w}$ is also du-opaque. Let $H$ be any finite opaque history such that for every pair of write operations write $e_{k}(X, v)$ and write w $_{m}(X, v)$, performed by transactions $T_{k}, T_{m} \in \operatorname{txns}(H)$, respectively, $v \neq v^{\prime}$.

Since $H$ is opaque, there is a final-state serialization $S$ of $H$. Suppose by contradiction that $H$ is not du-opaque. Thus, there is a $\operatorname{read}_{k}(X)$ that returns a value $v \in V$ in $S$ that is not legal in $S_{H}^{k, X}$, the local serialization of $\operatorname{read}_{k}(X)$ with respect to $H$ and $S$. Let $H^{k, X}$ and $S^{k, X}$ denote the prefixes of $H$ and $S$, respectively, up to the response of $\operatorname{read}_{k}(X)$ in $H$ and $S$. Recall that $S_{H}^{k, X}$, the local serialization of $\operatorname{read}_{k}(X)$ with respect to $H$ and $S$, is the subsequence of $S^{k, X}$ that does not contain events of any transaction $T_{i} \in \operatorname{txns}(H)$ so that the invocation of $\operatorname{try} C_{i}()$ is not in $H^{k, X}$. Since $\operatorname{read}_{k}(X)$ is legal in $S$, there is a committed transaction $T_{m} \in \operatorname{txns}(H)$ that performs write $e_{m}(X, v)$ that is the latest such write in $S$ that precedes $T_{k}$. Thus, if $\operatorname{read}_{k}(X)$ is not legal in $S_{H}^{k, X}$, the only possibility is that $\operatorname{read}_{k}(X) \prec_{H}^{R T} \operatorname{try} C_{m}()$. Under the assumption of unique writes, there does not exist any other transaction $T_{j} \in \operatorname{txns}(H)$ that performs write $_{j}(X, v)$. Consequently, there does not exist any $\bar{H}^{k, X}$ (some completion of $H^{k, X}$ ) and a t-complete t-sequential history $S^{\prime}$, such that $S^{\prime}$ is equivalent to $\bar{H}^{k, X}$ and $S^{\prime}$ contains any committed transaction that writes $v$ to $X$. This is, $H^{k, X}$ is not final-state opaque. However, since $H$ is opaque, every prefix of $H$ must be final-state opaque, which is a contradiction.

By Definition 3.3 an infinite history $H$ is opaque if every finite prefix of $H$ is final-state opaque. Theorem 3.5 now implies that Opacity $u$ w $\subseteq$ du-Opacity.

Definition 3.3 and Corollary 3.2 imply that du-Opacity $\subseteq$ Opacity $_{u w}$.

The sequential-history case The deferred-update semantics was mentioned by Guerraoui et al. 57 and later adopted by Kuznetsov and Ravi [88. In both papers, opacity is only defined for sequential histories, where every invocation of a t-operation is immediately followed by a matching response. In particular, these definitions require the final-state serialization to respect the read-commit order: in these definitions, a history $H$ is opaque if there is a final-state serialization $S$ of $H$ such that if a t-read of a t-object $X$ by a transaction $T_{k}$ precedes the tryC of a transaction $T_{m}$ that commits on $X$ in $H$, then $T_{k}$ precedes $T_{m}$ in $S$. As we observed in Figure 3.4 this definition is not equivalent to opacity even for sequential histories.

The property considered in [57, 88] is strictly stronger than du-opacity: the sequential history $H$ in Figure 3.4 is du-opaque (and consequently opaque by Theorem 3.10): a du-opaque serialization (in fact the only possible one) for this history is $T_{1}, T_{3}, T_{2}$. However, in the restriction of opacity defined above, $T_{2}$ must precede $T_{3}$ in any serialization, since the response of $\operatorname{read}_{2}(X)$ precedes the invocation of $\operatorname{try} C_{3}()$. 


\subsection{Strict serializability with DU semantics}

In this section, we discuss the deferred-update restriction of strict serializability from Definition 2.2 First, we remark that, just as final-state opacity, strict serializability is not prefix-closed (cf. Figure 3.1). However, we show that the restriction of deferred-update semantics applied to strict serializability induces a safety property.

Definition 3.6 (Strict serializability with du semantics). A finite history $H$ is strictly serializable if there is a legal t-complete $t$-sequential history $S$, such that

1. there is a completion $\bar{H}$ of $H$, such that $S$ is equivalent to $\operatorname{cseq}(\bar{H})$, where cseq $(\bar{H})$ is the subsequence of $\bar{H}$ reduced to committed transactions in $\bar{H}$,

2. for any two transactions $T_{k}, T_{m} \in$ txns $(H)$, if $T_{k} \prec_{H}^{R T} T_{m}$, then $T_{k}$ precedes $T_{m}$ in $S$, and

3. each $\operatorname{read}_{k}(X)$ in $S$ that does not return $A_{k}$ is legal in $S_{H}^{k, X}$.

Notice that every du-opaque history is strictly serializable, but not vice-versa.

Theorem 3.12. Strict serializability is a safety property.

Proof. Observe that any strictly serializable serialization of a finite history $H$ does not include events of any transaction that has not invoked $\operatorname{try} C$ in $H$.

To show prefix-closure, a proof almost identical to that of Lemma 3.1 implies that, given a strictly serializable history $H$ and a serialization $S$, there is a serialization $S^{\prime}$ of $H^{\prime}\left(H^{\prime}\right.$ is some prefix of $\left.H\right)$ such that $\operatorname{seq}\left(S^{\prime}\right)$ is a prefix of $\operatorname{seq}(S)$.

Consider an infinite sequence of finite histories

$$
H^{0}, \ldots, H^{i}, H^{i+1}, \ldots
$$

where $H^{i+1}$ is a one-event extension of $H^{i}$, we prove that the infinite limit $H$ of this ever-extending sequence is strictly serializable. As in Theorem 3.5, we construct an infinite rooted directed graph $G_{H}$ : a vertex is a tuple $\left(H^{i}, S^{i}\right)$ (note that for each $i \in \mathbb{N}$, there are several such vertices of this form), where $S^{i}$ is a serialization of $H^{i}$ and there is an edge from $\left(H^{i}, S^{i}\right)$ to $\left(H^{i+1}, S^{i+1}\right)$ if $\operatorname{seq}\left(S^{i}\right)$ is a prefix of $\operatorname{seq}\left(S^{i+1}\right)$. The resulting graph is finitely branching since the out-degree of a vertex is bounded by the number of possible serializations of a history. Observe that for every vertex $\left(H^{i+1}, S^{i+1}\right)$, there is a vertex $\left.H^{i}, S^{i}\right)$ such that $\operatorname{seq}\left(S^{i}\right)$ is a prefix of $\operatorname{seq}\left(S^{i+1}\right)$. Thus, $G_{H}$ is connected since we can iteratively construct a path from the root $\left(H^{0}, S^{0}\right)$ to every vertex $\left(H^{i}, S^{i}\right)$ in $G_{H}$. Applying König's Path Lemma to $G_{H}$, we obtain an infinite sequence of distinct vertices, $\left(H^{0}, S^{0}\right),\left(H^{1}, S^{1}\right), \ldots,\left(H^{i}, S^{i}\right), \ldots$ Then, $S=\lim _{i \rightarrow \infty} S_{i}$ gives the desired serialization of $H$.

\subsection{Du-opacity vs. other deferred-update criteria}

In this section, we first study two relaxations of opacity: Virtual-world consistency [83] and Transactional Memory Specification 1 [42]. We then study Transactional Memory Specification 2 which is a restriction of opacity. 


$$
T_{2} \stackrel{W_{2}(X, 1)}{\longmapsto} C_{2}
$$

Figure 3.5: A history that is du-VWC, but not du-opaque.

\subsubsection{Virtual-world consistency}

Virtual World Consistency (VWC) [83] was proposed as a relaxation of opacity (in our case, du-opacity), where each aborted transaction should be consistent with its causal past (but not necessarily with a serialization formed by committed transactions). Intuitively, a transaction $T_{1}$ causally precedes $T_{2}$ if $T_{2}$ reads a value written and committed by $T_{1}$. The original definition 83 required that no two write operations are ever invoked with the same argument (the unique-writes assumption). Therefore, the causal precedence is unambiguously identified for each transactional read. Below we give a more general definition.

Given a t-sequential legal history $S$ and transactions $T_{i}, T_{j} \in \operatorname{txns}(S)$, we say that $T_{i}$ reads $X$ from $T_{j}$ if (1) $T_{i}$ reads $v$ in $X$ and (2) $T_{j}$ is the last committed transaction that writes $v$ to $X$ and precedes $T_{i}$ in $S$.

Now consider a (not necessarily t-sequential) history $H$. We say that $T_{i}$ could have read $X$ from $T_{j}$ in $H$ if $T_{j}$ writes a value $v$ to a t-object $X, T_{i}$ reads $v$ in $X$, and $\operatorname{read}_{i}(X) \nprec_{H}^{R T} \operatorname{try} C_{j}()$.

Given $\mathcal{T} \subseteq \operatorname{txns}(H)$, let $H^{\mathcal{T}}$ denote the subsequence of $H$ restricted to events of transactions in $\mathcal{T}$.

Definition 3.7 (du-VWC). A finite history $H$ is du-virtual-world consistent if it is strictly serializable (with du-semantics), and for every aborted or t-incomplete transaction $T_{i} \in$ txns $(H)$, there is $\mathcal{T} \subseteq$ $\operatorname{txns}(H)$ including $T_{i}$ and a t-sequential t-complete legal history $S$ such that:

1. $S$ is equivalent to a completion of $H^{\mathcal{T}}$,

2. For all $T_{j}, T_{k} \in \operatorname{txns}(S)$, if $T_{j}$ reads $X$ from $T_{k}$ in $S$, then $T_{j}$ could have read $X$ from $T_{k}$ in $H$,

3. $S$ respects the per-process order of $H$ : if $T_{j}$ and $T_{k}$ are executed by the same process and $T_{j} \prec_{H}^{R T} T_{k}$, then $T_{j} \prec_{S} T_{k}$.

We refer to $S$ as a du-VWC serialization for $T_{i}$ in $H$.

Intuitively, with every t-read on $X$ performed by $T_{i}$ in $H$, the du-VWC serialization $S$ associates some transaction $T_{j}$ from which $T_{i}$ could have read the value of $X$. Recursively, with every read performed by $T_{j}, S$ associates some $T_{m}$ from which $T_{j}$ could have read, etc. Altogether, we get a "plausible" causal past of $T_{i}$ that constitutes a serial history. Notice that to ensure deferred-update semantics, we only allow a transaction $T_{j}$ to read from a transaction $T_{k}$ that invoked $\operatorname{try} C_{k}$ by the time of the read operation of $T_{j}$.

We now prove that du-VWC is a strictly weaker property than du-opacity. Since du-TMS2 is strictly weaker than du-opacity, it follows that Du-TMS2 $\varsubsetneqq \mathrm{du}-\mathrm{VWC}$.

Theorem 3.13. Du-opacity $\varsubsetneqq d u$ - $V W C$.

Proof. If a history $H$ is du-opaque, then there is a du-opaque serialization $S$ equivalent to $\bar{H}$, where $\bar{H}$ is some completion of $H$. By construction, $S$ is a total-order on the set of all transactions that participate in $S$. Trivially, by taking $\mathcal{T}=\operatorname{txns}(H)$, we derive that $S$ is a du-VWC serialization for every aborted or t-incomplete transaction $T_{i} \in \operatorname{txn} s(H)$. Indeed, $S$ respects the real-time order and, thus, the per-process order of $H$. Since $S$ respects the deferred-update order in $H$, every t-read in $S$ "could have happened" in $H$. 
To show that the inclusion is strict, Figure 3.5 depicts a history $H$ that is du-VWC, but not du-opaque. Clearly, $H$ is strictly serializable. Here $T_{2}, T_{1}$ is the required du-VWC serialization for aborted transaction $T_{1}$. However, $H$ has no du-opaque serialization.

Theorem 3.14. $D u-V W C$ is a safety property.

Proof. By Definition 3.7 a history $H$ is du-VWC if and only if $H$ is strictly serializable and there is a du-VWC serialization for every transaction $T_{i} \in \operatorname{txns}(H)$ that is aborted or t-incomplete in $H$.

To prove prefix-closure, recall that strict serializability is a prefix-closed property (Theorem 3.12). Therefore, any du-VWC serialization $S$ for a transaction $T_{i}$ in history $H$ is also a du-VWC serialization $S$ for a transaction $T_{i}$ in any prefix of $H$ that contains events of $T_{i}$.

To prove limit-closure, consider an infinite sequence of du-VWC histories $H^{0}, H^{1}, \ldots, H^{i}, H^{i+1}, \ldots$, where each $H^{i+1}$ is the one-event extension of $H^{i}$ and prove that the infinite limit, $H$ of this sequence is also a du-VWC history. Theorem 3.12 establishes that there is a strictly serializable serialization for $H$.

Since, for all $i \in \mathbb{N}, H^{i}$ is du-VWC, for every transaction $T_{i}$ that is t-incomplete or aborted in $H^{i}$, there is a VWC serialization for $T_{i}$. Consequently, there is a du-VWC serialization for every aborted or incomplete transaction $T_{i}$ in $H$.

\subsubsection{Transactional memory specification (TMS)}

Transactional Memory Specification (TMS) 1 and 2 were formulated in I/O automata [42]. Following [14], we adapt these definitions to our framework and explicitly introduce the deferred-update requirement.

TMS1. Given a history $H$, TMS1 requires us to justify the behavior of all committed transactions in $H$ by a legal t-complete t-sequential history that preserves the real-time order in $H$ (strict serializability), and to justify the response of each complete t-operation performed in $H$ by a legal t-complete t-sequential history $S$. The t-sequential history $S$ used to justify a complete t-operation $o p_{i, k}$ (the $i^{\text {th }}$ t-operation performed by transaction $T_{k}$ ) includes $T_{k}$ and a subset of transactions from $H$ whose operations justify $o p_{i, k}$. (Our description follows [14.)

Let $H^{k, i}$ denote the prefix of a history $H$ up to (and including) the response of $i^{\text {th }}$ t-operation $o p_{k, i}$ of transaction $T_{k}$. We say that a history $H^{\prime \prime}$ is a possible past of $H^{k, i}$ if $H^{\prime \prime}$ is a subsequence of $H^{k, i}$ and consists of all events of transaction $T_{k}$ and all events from some subset of committed transactions and transactions that have invoked $\operatorname{try} C$ in $H^{k, i}$ such that if a transaction $T \in H^{\prime \prime}$, then for a transaction $T^{\prime} \prec_{H^{k, i}}^{R T} T, T^{\prime} \in H^{\prime \prime}$ if and only if $T^{\prime}$ is committed in $H^{k, i}$. Let $\operatorname{cTMSpast}\left(H, o p_{k, i}\right)$ denote the set of possible pasts of $H^{k, i}$.

For any history $H^{\prime \prime} \in c T M \operatorname{Spast}\left(H, o p_{k, i}\right)$, let $\operatorname{ccomp}\left(H^{\prime \prime}\right)$ denote the history generated from $H^{\prime \prime}$ by the following procedure: for all $m \neq k$, replace every event $A_{m}$ by $C_{m}$ and complete every incomplete $\operatorname{try} C_{m}$ with including $C_{m}$ at the end of $H^{\prime \prime}$; include $\operatorname{try} C_{k} \cdot A_{k}$ at the end of $H^{\prime \prime}$.

Definition 3.8 (du-TMS1). A history $H$ satisfies du-TMS1 if

1. $H$ is strictly serializable (with du-semantics), and

2. for each complete $t$-read op $p_{i, k}$ that returns a non- $A_{k}$ response in $H$, there exist a legal $t$-complete $t$-sequential history $S$ and a history $H^{\prime}$ such that:

- $H^{\prime}=\operatorname{ccomp}\left(H^{\prime \prime}\right)$, where $H^{\prime \prime} \in \operatorname{cTMSpast}\left(H\right.$, op $\left._{k, i}\right)$

- $H^{\prime}$ is equivalent to $S$

- for any two transactions $T_{k}$ and $T_{m}$ in $H^{\prime}$, if $T_{k} \prec_{H^{\prime}}^{R T} T_{m}$ then $T_{k}<_{S} T_{m}$

We refer to $S$ as the du-TMS1 serialization for $o p_{i, k}$.

Theorem 3.15. Du-TMS1 is a safety property. 


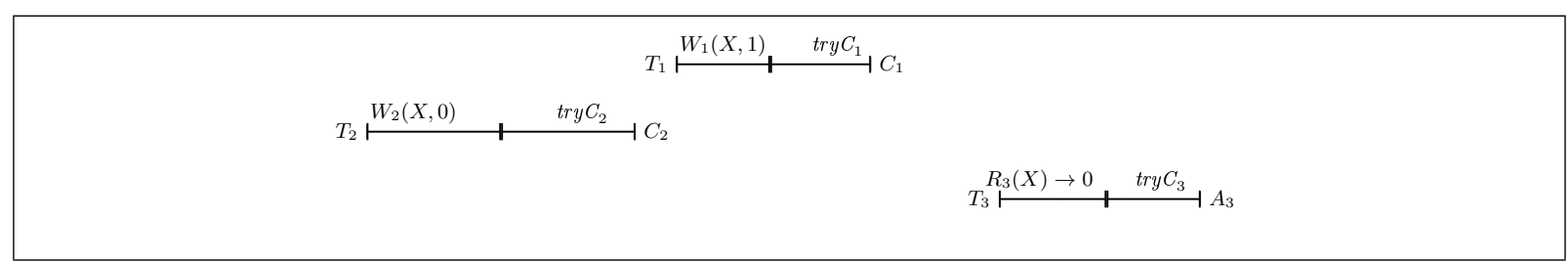

Figure 3.6: A history which is du-VWC but not du-TMS1.

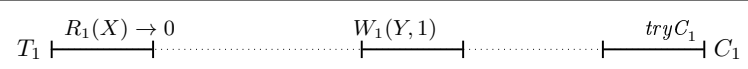

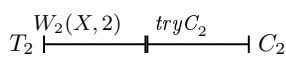

$$
\begin{aligned}
& T_{3} \stackrel{R_{3}(X) \rightarrow 0}{\longmapsto} \longmapsto A_{3} \\
& T_{4} \stackrel{R_{4}(X) \rightarrow 2}{R_{4}(Y) \rightarrow 0} \longmapsto
\end{aligned}
$$

Figure 3.7: A history which is du-TMS1 but not du-VWC.

Proof. A history $H$ is du-TMS1 if and only if $H$ is strictly serializable and there is a du-TMS1 serialization for every t-operation $o p_{k, i}$ that does not return $A_{k}$ in $H$.

To see that du-TMS1 is prefix closed, recall that strict serializability is a prefix-closed property. Let $H$ be any du-TMS1 history and $H^{i}$, any prefix of $H$. We now need to prove that, for every t-operation $o p_{k, i} \neq \operatorname{try} C_{k}$ that returns a non- $A_{k}$ response in $H^{i}$, there is a du-TMS1 serialization for $o p_{k, i}$. But this is immediate since the du-TMS1 serialization for $o p_{i, k}$ in $H$ is also the required du-TMS1 serialization for $o p_{k, i}$ in $H^{i}$.

To see that du-TMS1 is limit closed, consider an infinite sequence

$$
H^{0}, H^{1}, \ldots H^{i}, H^{i+1}, \ldots
$$

of finite du-TMS1 histories, such that $H^{i+1}$ is a one-event extension of $H^{i}$. Let let $H$ be the corresponding infinite limit history. We want to show that $H$ is also du-TMS1.

Since strict serializability is a limit-closed property (Theorem 3.12), $H$ is strictly serializable. By assumption, for all $i \in \mathbb{N}, H^{i}$ is du-TMS1. Thus, for every transaction $T_{i}$ that participates in $H^{i}$, there is a du-TMS1 serialization $S^{i, k}$ for each t-operation $o p_{k, i}$. But $S^{i, k}$ is also the required du-TMS1 serialization for $o p_{k, 1}$ in $H$. The claim follows.

It has been shown [95] that Opacity is a strictly stronger property than du-TMS1, that is, Opacity $\varsubsetneqq$ du-TMS1. Since Du-Opacity $\varsubsetneqq$ Opacity (Theorem 3.10 it follows that Du-Opacity $\varsubsetneqq$ du-TMS1. On the other hand, du-TMS1 is incomparable to du-VWC, as demonstrated by the following examples.

Proposition 3.2. There is a history that is du-TMS1, but not du-VWC.

Proof. Figure 3.7 depicts a history $H$ that is du-TMS1, but not du-VWC. Observe that $H$ is strictly serializable. To prove that $H$ is du-TMS1, we need to prove that there is a TMS1 serialization for each t-read that returns a non-abort response in $H$. Clearly, the serialization in which only $T_{3}$ participates is the required TMS1 serialization for $\operatorname{read}_{3}(X) \rightarrow 0$. Now consider the aborted transaction $T_{4}$. The TMS1 serialization for $\operatorname{read}_{4}(X) \rightarrow 2$ is $T_{2}, T_{4}$, while the TMS1 serialization that justifies the response of $\operatorname{read}_{4}(Y)->0$ includes just $T_{4}$ itself. The only nontrivial t-read whose response needs to be justified is $\operatorname{read}_{4}(Z) \rightarrow 3$. Indeed, $\operatorname{try} C_{3}$ overlaps with $\operatorname{read}_{4}(Z)$ and thus, the response of $\operatorname{read}_{4}(Z)$ can be justified by choosing transactions in $c$ TMSpart $\left(H, \operatorname{read}_{4}(Z)\right)$ to be $\left\{T_{3}, T_{2}, T_{4}\right\}$ and then deriving a TMS1 serialization $S=T_{3}, T_{2}, T_{4}$ for $\operatorname{read}_{4}(Z) \rightarrow 3$ in which $\operatorname{try} C_{3}$ may be completed by including the commit response.

However, $H$ is not du-VWC. Consider transaction $T_{3}$ which returns $A_{3}$ in $H: T_{3}$ must be aborted in any serialization equivalent to some direct causal past of $T_{4}$. But $\operatorname{read}_{4}(Z)$ returns the value 3 that is written by $T_{3}$. Thus, $\operatorname{read}_{4}(Z)$ cannot be legal in any du-VWC serialization for $T_{4}$. 


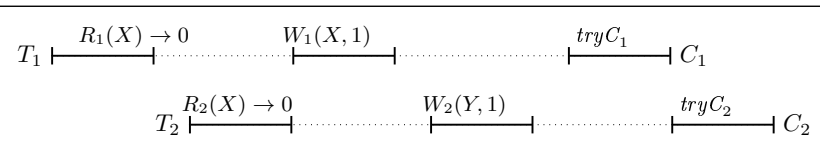

Figure 3.8: A history that is du-opaque, but not TMS2 [42].

Proposition 3.3. There is a history that is $d u$-VWC, but not du-TMS1.

Proof. Figure 3.6 depicts a history $H$ that is du-VWC, but not du-TMS1. Clearly, $H$ is strictly serializable. Observe that $T_{3}$ could have read only from $T_{1}$ in $H$ ( $T_{1}$ writes the value 0 to $X$ that is returned by read $\left._{3}(X)\right)$. Therefore, $T_{1}, T_{3}$ is the required du-VWC serialization for aborted transaction $T_{3}$.

However, $H$ is not du-TMS1: since both transactions $T_{1}$ and $T_{2}$ are committed and precede $T_{3}$ in realtime order, they must be included in any du-TMS1 serialization for $\operatorname{read}_{3}(X) \rightarrow 0$. But there is no such du-TMS1 serialization that would ensure the legality of $\operatorname{read}_{3}(X)$.

TMS2. We now study the TMS2 definition which imposes an extra restriction on the opaque serialization.

Definition 3.9 (du-TMS2). A history $H$ is du-TMS2 if there is a legal $t$-complete t-sequential history $S$ equivalent to some completion, $\bar{H}$ of $H$ such that

1. for any two transactions $T_{k}, T_{m} \in$ txns $(H)$, such that $T_{m}$ is a committed updating transaction, if $C_{k} \prec_{H}^{R T} \operatorname{try} C_{m}$ or $A_{k} \prec_{H}^{R T} \operatorname{try} C_{m}$, then $T_{k} \prec_{S} T_{m}$, and

2. for any two transactions $T_{k}, T_{m} \in \operatorname{txns}(H)$, if $T_{k} \prec_{H}^{R T} T_{m}$, then $T_{k}<_{S} T_{m}$, and

3. each read $(X)$ in $S$ that does not return $A_{k}$ is legal in $S_{H}^{k, X}$.

We refer to $S$ as the du-TMS2 serialization of $H$.

It has been shown 95 that TMS2 is a strictly stronger property than Opacity, i.e., TMS2 $\subsetneq$ Opacity. We now show that du-TMS2 is strictly stronger than du-opacity. Indeed, from Definition 3.9 , we observe that every history that is du-TMS2 is also du-opaque. The following proposition completes the proof.

Proposition 3.4. There is a history that is du-opaque, but not du-TMS2.

Proof. Figure 3.8 depicts a history $H$ that is du-opaque, but not du-TMS2. Indeed, there is a du-opaque serialization $S$ of $H$ such that $\operatorname{seq}(S)=T_{2}, T_{1}$. On the other hand, since $T_{1}$ commits before $T_{2}, T_{1}$ must precede $T_{2}$ in any du-TMS2 serialization, there does not exist any such serialization that ensures every t-read is legal. Thus, $H$ is not du-TMS2.

Theorem 3.16. Du-TMS2 is prefix-closed.

Proof. Let $H$ be any du-TMS2 history. Then, $H$ is also du-opaque. By Corollary 3.2 , for every $i \in \mathbb{N}$, there is a du-opaque serialization $S^{i}$ for $H^{i}$. We now need to prove that, for any two transactions $T_{k}, T_{m} \in \operatorname{txns}\left(H^{i}\right)$, such that $T_{m}$ is a committed updating transaction, if $C_{k} \prec_{H^{i}}^{R T} \operatorname{try} C_{m}$ or $A_{k} \prec_{H^{i}}^{R T}$ $\operatorname{try} C_{m}$, there is a du-opaque serialization $S^{i}$ with the restriction that $T_{k} \prec_{S^{i}} T_{m}$.

Suppose by contradiction that there exist transactions $T_{k}, T_{m} \in \operatorname{txns}\left(H^{i}\right)$, such that $T_{m}$ is a committed updating transaction and $C_{k} \prec_{H^{i}}^{R T} \operatorname{try} C_{m}$ or $A_{k} \prec_{H^{i}}^{R T} \operatorname{try} C_{m}$, but $T_{m}$ must precede $T_{k}$ in any du-opaque serialization $S^{i}$. Since $T_{m} \nprec_{H^{i}}^{R T} T_{k}$, the only possibility is that $T_{m}$ performs write $_{m}(X, v)$ and there is $\operatorname{read}_{k}(X) \rightarrow v$. However, by our assumption, write $k(X, v) \prec_{H^{i}}^{R T} \operatorname{try} C_{m}$ : thus, read $d_{k}(X)$ is not legal in its local serialization with respect to $H^{i}$ and $S^{i}$-contradicting the assumption that $S^{i}$ is a du-opaque serialization of $H^{i}$. Thus, there is a du-TMS2 serialization for $H^{i}$, proving that du-TMS2 is a prefix-closed property. 


\begin{tabular}{l|l|l|l|l|} 
& du-opacity & du-VWC & du-TMS1 & du-TMS2 \\
\hline du-opacity & & $\varsubsetneqq$ & $\varsubsetneqq$ & $\supsetneqq$ \\
\hline du-VWC & $\supsetneqq$ & & $\times$ & $\supsetneqq$ \\
\hline du-TMS1 & $\supsetneqq$ & $\times$ & & $\supsetneqq$ \\
\hline du-TMS2 & $\varsubsetneqq$ & $\varsubsetneqq$ & $\varsubsetneqq$ &
\end{tabular}

Table 3.1: Relations between TM consistency definitions.

Proposition 3.5. Du-TMS2 is not limit-closed.

Proof. The counter-example to establish that du-opacity is not limit-closed (Figure 3.2 ) also shows that du-TMS2 is not limit-closed: all histories discussed in the counter-example are in du-TMS2.

\subsection{Related work and Discussion}

The properties discussed in this chapter explicitly preclude reading from a transaction that has not yet invoked tryCommit, which makes them prefix-closed and facilitates their verification. We believe that this constructive definition is useful to TM practitioners, since it streamlines possible implementations of t-read and tryCommit operations.

We showed that du-opacity is limit-closed under the restriction that every operation eventually terminates, while du-VWC and du-TMS1 are (unconditionally) limit-closed, which makes them safety properties 97 .

Table 3.1 summarizes the containment relations between the properties discussed in this chapter: opacity, du-opacity, du-VWC, du-TMS1 and du-TMS2. For example, "du-opacity $\subsetneq$ opacity" means that the set of du-opaque histories is a proper subset of the set of opaque histories, i.e., du-opacity is a strictly stronger property than opacity. Incomparable (not related by containment) properties, such as du-TMS1 and du-VWC are marked with $\times$.

Linearizability [26, 81, when applied to objects with finite nondeterminism (i.e., an operation applied to a given state may produce only finitely many outcomes) sequential specifications is a safety property [64, 97. Recently, it has been shown [64 that linearizability is not limit-closed if the implemented object may expose infinite non-determinism [64, that is, an operation applied to a given state may produce infinitely many different outcomes. The limit-closure proof (cf. Theorem 3.5), using König's lemma, cannot be applied with infinite non-determinism, because the out-degree of the graph $G_{H}$, constructed for the limit infinite history $H$, is not finite.

In contrast, the TM abstraction is deterministic, since reads and writes behave deterministically in serial executions, yet du-opacity is not limit-closed. It turns out that the graph $G_{H}$ for the counterexample history $H$ in Figure 3.2 is not connected. For example, one of the finite prefixes of $H$ can be serialized as $T_{3}, T_{1}, T_{2}$, but no prefix has a serialization $T_{3}, T_{1}$ and, thus, the root is not connected to the corresponding vertex of $G_{H}$. Thus, the precondition of König's lemma does not hold for $G_{H}$ : the graph is in fact an infinite set of isolated vertices. This is because du-opacity requires even incomplete reading transactions, such as $T_{2}$, to appear in the serialization, which is not the case for linearizability, where incomplete operations may be removed from the linearization. 


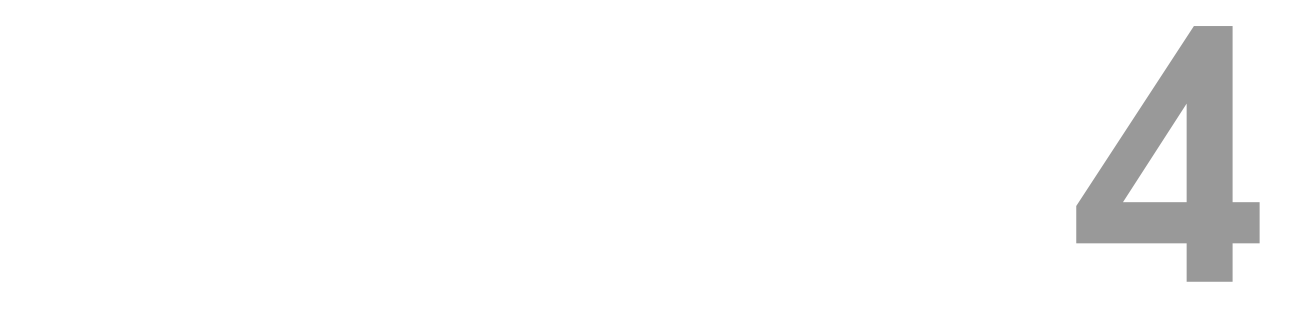

\section{Complexity bounds for blocking TMs}

"I can’t believe that!" said Alice "Can't you?" the Queen said in a pitying tone. "Try again: draw a long breath, and shut your eyes." Alice laughed. "There's no use trying," she said: "one can't believe impossible things." "I daresay you haven't had much practice," said the Queen. "When I was your age, I always did it for half-an-hour a day. Why, sometimes I've believed as many as six impossible things before breakfast."

Lewis Carroll-Through the Looking-Glass

\subsection{Overview}

In this chapter, we present complexity bounds for TM implementations that provide no non-blocking progress guarantees for transactions and typically allow a transaction to block (delay) or abort in concurrent executions. We refer to Section 2.7 in Chapter 2 for an overview of the complexity metrics considered in the thesis.

Sequential TMs. We start by presenting complexity bounds for single-lock TMs that satisfy sequential TM-progress. We show that a read-only transaction in an opaque TM featured with weak DAP, weak invisible reads, ICF TM-liveness and sequential TM-progress must incrementally validate every next read operation. This results in a quadratic (in the size of the transaction's read set) step-complexity lower bound. Secondly, we prove that if the TM-correctness property is weakened to strict serializability, there exist executions in which the tryCommit of some transaction must access a linear (in the size of the transaction's read set) number of distinct base objects. We then show that expensive synchronization 
in TMs cannot be eliminated: even single-lock TMs must perform a RAW (read-after-write) or AWAR (atomic-write-after-read) pattern [16].

Progressive TMs. We turn our focus to progressive TM implementations which allow a transaction to be aborted only due to read-write conflicts with concurrent transactions. We introduce a new metric called protected data size that, intuitively, captures the amount of data that a transaction must exclusively control at some point of its execution. All progressive TM implementations we are aware of (see, e.g., an overview in [60]) use locks or timing assumptions to give an updating transaction exclusive access to all objects in its write set at some point of its execution. For example, lock-based progressive implementations like TL [39] and TL2 [38] require that a transaction grabs all locks on its write set before updating the corresponding base objects. Our result shows that this is an inherent price to pay for providing progressive concurrency: every committed transaction in a progressive and strict DAP TM implementation providing starvation-free TM-liveness must, at some point of its execution, protect every t-object in its write set.

We also present a very cheap progressive opaque strict DAP TM implementation from read-write base objects with constant expensive synchronization and constant memory stall complexities.

Strongly progressive TMs. We then prove that in any strongly progressive strictly serializable TM implementation that accesses the shared memory with read, write and conditional primitives, such as compare-and-swap and load-linked/store-conditional, the total number of remote memory references (RMRs) that take place in an execution of a progressive TM in which $n$ concurrent processes perform transactions on a single t-object might reach $\Omega(n \log n)$. The result is obtained via a reduction to an analogous lower bound for mutual exclusion [21. In the reduction, we show that any TM with the above properties can be used to implement a deadlock-free mutual exclusion, employing transactional operations on only one t-object and incurring a constant RMR overhead. The lower bound applies to RMRs in both the cache-coherent $(C C$ ) and distributed shared memory (DSM) models, and it appears to be the first RMR complexity lower bound for transactional memory.

We also present a constant expensive synchronization strongly progressive TM implementation from readwrite base objects. Our implementation provides starvation-free TM-liveness, thus showing one means of circumventing the lower bound of Rachid et al. 62] who proved the impossibility of implementing strongly progressive strictly serializable TMs providing wait-free TM-liveness from read-write base objects.

Permissive TMs. We conclude our study of blocking TMs by establishing a linear (in the transaction's data set size) separation between the worst-case transaction expensive synchronization complexity of strongly progressive TMs and permissive TMs that allow a transaction to abort only if committing it would violate opacity. Specifically, we show that an execution of a transaction in a permissive opaque TM implementation that provides starvation-free TM-liveness may require to perform at least one RAW/AWAR pattern per t-read.

Roadmap of Chapter 4. Section 4.2 studies "single-lock" TMs that provide minimal progressiveness or sequential TM-progress, Section 4.3 is devoted to progressive TMs while Section 4.4 is on strongly progressive TMs. In Section 4.5, we study the cost of permissive TMs that allow a transaction to abort only if committing it would violate opacity. Finally, we present related work and open questions in Section 4.6.

\subsection{Sequential TMs}

We begin with "single-lock", i.e., sequential TMs. Our first result proves that a read-only transaction in a sequential TM featured with weak DAP and weak invisible reads must incur the cost of validating its read set. This results in a quadratic (and resp., linear) (in the size of the transaction's read set) step-complexity lower bound if we assume opacity (and resp., strict serializability). Secondly, we show that expensive synchronization cannot be avoided even in such sequential TMs, i.e., a serializable TM must perform a RAW/AWAR even when transactions are guaranteed to commit only in the absence of any concurrency. 


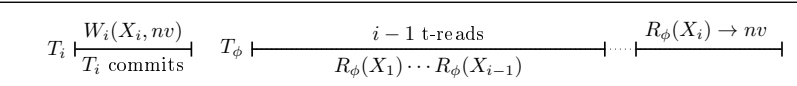

(a) $R_{\phi}\left(X_{i}\right)$ must return $n v$ by strict serializability

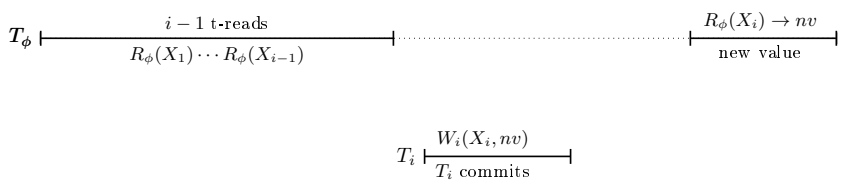

(b) $T_{i}$ does not observe any conflict with $T_{\phi}$

Figure 4.1: Executions in the proof of Lemma 4.1 By weak DAP, $T_{\phi}$ cannot distinguish this from the execution in Figure $4.1 \mathrm{a}$

We first prove the following auxiliary lemma that will be of use in subsequent proofs.

Lemma 4.1. Let $M$ be any strictly serializable, weak DAP TM implementation that provides sequential TM-progress and sequential TM-liveness. Then, for all $i \in \mathbb{N}, M$ has an execution of the form $\pi^{i-1} \cdot \rho^{i} \cdot \alpha^{i}$ where,

- $\pi^{i-1}$ is the complete step contention-free execution of read-only transaction $T_{\phi}$ that performs $(i-1)$ $t$-reads: $\operatorname{read}_{\phi}\left(X_{1}\right) \cdots \operatorname{read}_{\phi}\left(X_{i-1}\right)$,

- $\rho^{i}$ is the t-complete step contention-free execution of a transaction $T_{i}$ that writes $n v \neq v$ to $X_{i}$ and commits ( $v$ is the initial value of $X-i$ ),

- $\alpha_{i}$ is the complete step contention-free execution fragment of $T_{\phi}$ that performs its $i^{\text {th }}$ t-read: $\operatorname{read}_{\phi}\left(X_{i}\right) \rightarrow n v_{i}$.

Proof. By sequential TM-progress and sequential TM-liveness, $M$ has an execution of the form $\rho^{i} \cdot \pi^{i-1}$. Since $\operatorname{Dset}\left(T_{k}\right) \cap \operatorname{Dset}\left(T_{i}\right)=\emptyset$ in $\rho^{i} \cdot \pi^{i-1}$, by Lemma 2.10, transactions $T_{\phi}$ and $T_{i}$ do not contend on any base object in execution $\rho^{i} \cdot \pi^{i-1}$. Thus, $\rho^{i} \cdot \pi^{i-1}$ is also an execution of $M$.

By assumption of strict serializability, $\rho^{i} \cdot \pi^{i-1} \cdot \alpha_{i}$ is an execution of $M$ in which the t-read of $X_{i}$ performed by $T_{\phi}$ must return $n v$. But $\rho^{i} \cdot \pi^{i-1} \cdot \alpha_{i}$ is indistinguishable to $T_{\phi}$ from $\pi^{i-1} \cdot \rho^{i} \cdot \alpha_{i}$. Thus, $M$ has an execution of the form $\pi^{i-1} \cdot \rho^{i} \cdot \alpha_{i}$.

\subsubsection{A quadratic lower bound on step complexity}

In this section, we present our step complexity lower bound for sequential TMs.

Theorem 4.2. For every weak DAP TM implementation $M$ that provides ICF TM-liveness, sequential TM-progress and uses weak invisible reads,

(1) If $M$ is opaque, for every $m \in \mathbb{N}$, there exists an execution $E$ of $M$ such that some transaction $T \in \operatorname{txns}(E)$ performs $\Omega\left(m^{2}\right)$ steps, where $m=\left|\operatorname{Rset}\left(T_{k}\right)\right|$.

(2) if $M$ is strictly serializable, for every $m \in \mathbb{N}$, there exists an execution $E$ of $M$ such that some transaction $T_{k} \in \operatorname{txns}(E)$ accesses at least $m-1$ distinct base objects during the executions of the $m^{\text {th }}$ t-read operation and try $C_{k}()$, where $m=\left|\operatorname{Rset}\left(T_{k}\right)\right|$.

Proof. For all $i \in\{1, \ldots, m\}$, let $v$ be the initial value of t-object $X_{i}$.

(1) Suppose that $M$ is opaque. Let $\pi^{m}$ denote the complete step contention-free execution of a transaction $T_{\phi}$ that performs $m$ t-reads: $\operatorname{read}_{\phi}\left(X_{1}\right) \cdots \operatorname{read}_{\phi}\left(X_{m}\right)$ such that for all $i \in\{1, \ldots, m\}, \operatorname{read}_{\phi}\left(X_{i}\right) \rightarrow v$.

By Lemma 4.1 for all $i \in\{2, \ldots, m\}, M$ has an execution of the form $E^{i}=\pi^{i-1} \cdot \rho^{i} \cdot \alpha_{i}$. 
For each $i \in\{2, \ldots, m\}, j \in\{1,2\}$ and $\ell \leq(i-1)$, we now define an execution of the form $\mathbb{E}_{j \ell}^{i}=$ $\pi^{i-1} \cdot \beta^{\ell} \cdot \rho^{i} \cdot \alpha_{j}^{i}$ as follows:

- $\beta^{\ell}$ is the t-complete step contention-free execution fragment of a transaction $T_{\ell}$ that writes $n v_{\ell} \neq v$ to $X_{\ell}$ and commits

- $\alpha_{1}^{i}$ (and resp. $\alpha_{2}^{i}$ ) is the complete step contention-free execution fragment of $\operatorname{read}_{\phi}\left(X_{i}\right) \rightarrow v($ and resp. $\left.\operatorname{read}_{\phi}\left(X_{i}\right) \rightarrow A_{\phi}\right)$.

Claim 4.3. For all $i \in\{2, \ldots, m\}$ and $\ell \leq(i-1), M$ has an execution of the form $\mathbb{E}_{1 \ell}^{i}$ or $\mathbb{E}_{2 \ell}^{i}$.

Proof. For all $i \in\{2, \ldots, m\}, \pi^{i-1}$ is an execution of $M$. By assumption of weak invisible reads and sequential TM-progress, $T_{\ell}$ must be committed in $\pi^{i-1} \cdot \rho^{\ell}$ and $M$ has an execution of the form $\pi^{i-1} \cdot \beta^{\ell}$. By the same reasoning, since $T_{i}$ and $T_{\ell}$ have disjoint data sets, $M$ has an execution of the form $\pi^{i-1} \cdot \beta^{\ell} \cdot \rho^{i}$.

Since the configuration after $\pi^{i-1} \cdot \beta^{\ell} \cdot \rho^{i}$ is quiescent, by ICF TM-liveness, $\pi^{i-1} \cdot \beta^{\ell} \cdot \rho^{i}$ extended with $\operatorname{read}_{\phi}\left(X_{i}\right)$ must return a matching response. If $\operatorname{read}_{\phi}\left(X_{i}\right) \rightarrow v_{i}$, then clearly $\mathbb{E}_{1}^{i}$ is an execution of $M$ with $T_{\phi}, T_{i-1}, T_{i}$ being a valid serialization of transactions. If $\operatorname{read}_{\phi}\left(X_{i}\right) \rightarrow A_{\phi}$, the same serialization justifies an opaque execution.

Suppose by contradiction that there exists an execution of $M$ such that $\pi^{i-1} \cdot \beta^{\ell} \cdot \rho^{i}$ is extended with the complete execution of $\operatorname{read}_{\phi}\left(X_{i}\right) \rightarrow r ; r \notin\left\{A_{\phi}, v\right\}$. The only plausible case to analyse is when $r=n v$. Since $\operatorname{read}_{\phi}\left(X_{i}\right)$ returns the value of $X_{i}$ updated by $T_{i}$, the only possible serialization for transactions is $T_{\ell}, T_{i}, T_{\phi}$; but $\operatorname{read}_{\phi}\left(X_{\ell}\right)$ performed by $T_{k}$ that returns the initial value $v$ is not legal in this serialization - contradiction.

We now prove that, for all $i \in\{2, \ldots, m\}, j \in\{1,2\}$ and $\ell \leq(i-1)$, transaction $T_{\phi}$ must access $(i-1)$ different base objects during the execution of $\operatorname{read}_{\phi}\left(X_{i}\right)$ in the execution $\pi^{i-1} \cdot \beta^{\ell} \cdot \rho^{i} \cdot \alpha_{j}^{i}$.

By the assumption of weak invisible reads, the execution $\pi^{i-1} \cdot \beta^{\ell} \cdot \rho^{i} \cdot \alpha_{j}^{i}$ is indistinguishable to transactions $T_{\ell}$ and $T_{i}$ from the execution $\tilde{\pi}^{i-1} \cdot \beta^{\ell} \cdot \rho^{i} \cdot \alpha_{j}^{i}$, where $R \operatorname{set}\left(T_{\phi}\right)=\emptyset$ in $\tilde{\pi}^{i-1}$. But transactions $T_{\ell}$ and $T_{i}$ are disjoint-access in $\tilde{\pi}^{i-1} \cdot \beta^{\ell} \cdot \rho^{i}$ and by Lemma 2.10 they cannot contend on the same base object in this execution.

Consider the $(i-1)$ different executions: $\pi^{i-1} \cdot \beta^{1} \cdot \rho^{i}, \ldots, \pi^{i-1} \cdot \beta^{i-1} \cdot \rho^{i}$. For all $\ell, \ell^{\prime} \leq(i-1) ; \ell^{\prime} \neq \ell, M$ has an execution of the form $\pi^{i-1} \cdot \beta^{\ell} \cdot \rho^{i} \cdot \beta^{\ell^{\prime}}$ in which transactions $T_{\ell}$ and $T_{\ell^{\prime}}$ access mutually disjoint data sets. By weak invisible reads and Lemma 2.10 the pairs of transactions $T_{\ell^{\prime}}, T_{i}$ and $T_{\ell^{\prime}}, T_{\ell}$ do not contend on any base object in this execution. This implies that $\pi^{i-1} \cdot \beta^{\ell} \cdot \beta^{\ell^{\prime}} \cdot \rho^{i}$ is an execution of $M$ in which transactions $T_{\ell}$ and $T_{\ell^{\prime}}$ each apply nontrivial primitives to mutually disjoint sets of base objects in the execution fragments $\beta^{\ell}$ and $\beta^{\ell^{\prime}}$ respectively (by Lemma 2.10 .

This implies that for any $j \in\{1,2\}, \ell \leq(i-1)$, the configuration $C^{i}$ after $E^{i}$ differs from the configurations after $\mathbb{E}_{j \ell}^{i}$ only in the states of the base objects that are accessed in the fragment $\beta^{\ell}$. Consequently, transaction $T_{\phi}$ must access at least $i-1$ different base objects in the execution fragment $\pi_{j}^{i}$ to distinguish configuration $C^{i}$ from the configurations that result after the $(i-1)$ different executions $\pi^{i-1} \cdot \beta^{1} \cdot \rho^{i}$, $\ldots, \pi^{i-1} \cdot \beta^{i-1} \cdot \rho^{i}$ respectively.

Thus, for all $i \in\{2, \ldots, m\}$, transaction $T_{\phi}$ must perform at least $i-1$ steps while executing the $i^{\text {th }}$ t-read in $\pi_{j}^{i}$ and $T_{\phi}$ itself must perform $\sum_{i=1}^{m-1} i=\frac{m(m-1)}{2}$ steps.

(2) Suppose that $M$ is strictly serializable, but not opaque. Since $M$ is strictly serializable, by Lemma 4.1 . it has an execution of the form $E=\pi^{m-1} \cdot \rho^{m} \cdot \alpha_{m}$.

For each $\ell \leq(i-1)$, we prove that $M$ has an execution of the form $E_{\ell}=\pi^{m-1} \cdot \beta^{\ell} \cdot \rho^{m} \cdot \bar{\alpha}^{m}$ where $\bar{\alpha}^{m}$ is the complete step contention-free execution fragment of $\operatorname{read}_{\phi}\left(X_{m}\right)$ followed by the complete execution of $\operatorname{try} C_{\phi}$. Indeed, by weak invisible reads, $\pi^{m-1}$ does not contain any nontrivial events and the execution $\pi^{m-1} \cdot \beta^{\ell} \cdot \rho^{m}$ is indistinguishable to transactions $T_{\ell}$ and $T_{m}$ from the executions $\tilde{\pi}^{m-1} \cdot \beta^{\ell}$ and $\tilde{\pi}^{m-1} \cdot \beta^{\ell} \cdot \rho^{m}$ respectively, where $\operatorname{Rset}\left(T_{\phi}\right)=\emptyset$ in $\tilde{\pi}^{m-1}$. Thus, applying Lemma 2.10 transactions 
$\beta^{\ell} \cdot \rho^{m}$ do not contend on any base object in the execution $\pi^{m-1} \cdot \beta^{\ell} \cdot \rho^{m}$. By ICF TM-liveness, read $_{\phi}\left(X_{m}\right)$ and $\operatorname{try} C_{\phi}$ must return matching responses in the execution fragment $\bar{\alpha}^{m}$ that extends $\pi^{m-1} \cdot \beta^{\ell} \cdot \rho^{m}$. Consequently, for each $\ell \leq(i-1), M$ has an execution of the form $E_{\ell}=\pi^{m-1} \cdot \beta^{\ell} \cdot \rho^{m} \cdot \bar{\alpha}^{m}$ such that transactions $T_{\ell}$ and $T_{m}$ do not contend on any base object.

Strict serializability of $M$ means that if $\operatorname{read}_{\phi}\left(X_{m}\right) \rightarrow n v$ in the execution fragment $\bar{\alpha}^{m}$, then $\operatorname{try} C_{\phi}$ must return $A_{\phi}$. Otherwise if $\operatorname{read}_{\phi}\left(X_{m}\right) \rightarrow v$ (i.e. the initial value of $X_{m}$ ), then $\operatorname{try} C_{\phi}$ may return $A_{\phi}$ or $C_{\phi}$.

Thus, as with (1), in the worst case, $T_{\phi}$ must access at least $m-1$ distinct base objects during the executions of $\operatorname{read}_{\phi}\left(X_{m}\right)$ and $\operatorname{try} C_{\phi}$ to distinguish the configuration $C^{i}$ from the configurations after the $m-1$ different executions $\pi^{m-1} \cdot \beta^{1} \cdot \rho^{m}, \ldots, \pi^{m-1} \cdot \beta^{m-1} \cdot \rho^{m}$ respectively.

\subsubsection{Expensive synchronization in Transactional memory cannot be eliminated}

In this section, we show that serializable TMs must perform a RAW/AWAR even if they are guaranteed to commit only when they run in the absence of any concurrency.

Theorem 4.4. Let $M$ be a serializable TM implementation providing sequential TM-progress and sequential TM-liveness. Then, every execution of $M$ in which a transaction running $t$-sequentially performs at least one t-read and at least one t-write contains a $R A W / A W A R$ pattern.

Proof. Consider an execution $\pi$ of $M$ in which a transaction $T_{1}$ running t-sequentially performs (among other events) $\operatorname{read}_{1}(X)$, write $1(Y, v)$ and $\operatorname{try} C_{1}()$. Since $M$ satisfies sequential TM-progress and sequential TM-liveness, $T_{1}$ must commit in $\pi$. Clearly $\pi$ must contain a write to a base object. Otherwise a subsequent transaction reading $Y$ would return the initial value of $Y$ instead of the value written by $T_{1}$.

Let $\pi_{w}$ be the first write to a base object in $\pi$. Thus, $\pi$ can be represented as $\pi_{s} \cdot \pi_{w} \cdot \pi_{f}$.

Now suppose by contradiction that $\pi$ contains neither RAW nor AWAR patterns.

Since $\pi_{s}$ contains no writes, the states of base objects in the initial configuration and in the configuration after $\pi_{s}$ is performed are the same. Consider an execution $\pi_{s} \cdot \rho$ where in $\rho$, a transaction $T_{2}$ performs $\operatorname{read}_{2}(Y)$, write $2(X, 1), \operatorname{try} C_{2}()$ and commits. Such an execution exists, since $\rho$ is indistinguishable to $T_{2}$ from an execution in which $T_{2}$ runs t-sequentially and thus $T_{2}$ cannot be aborted in $\pi_{s} \cdot \rho$.

Since $\pi_{w}$ contains no AWAR, $\pi_{s} \cdot \rho \cdot \pi_{w}$ is an execution of $M$.

Since $\pi_{w} \cdot \pi_{f}$ contains no RAWs, every read performed in $\pi_{w} \cdot \pi_{f}$ is applied to base objects which were previously written in $\pi_{w} \cdot \pi_{f}$. Thus, there exists an execution $\pi_{s} \cdot \rho \cdot \pi_{w} \cdot \pi_{f}$, such that $T_{1}$ cannot distinguish $\pi_{s} \cdot \pi_{w} \cdot \pi_{f}$ and $\pi_{s} \cdot \rho \cdot \pi_{w} \cdot \pi_{f}$. Hence, $T_{1}$ commits in $\pi_{s} \cdot \rho \cdot \pi_{w} \cdot \pi_{f}$.

But $T_{1}$ reads the initial value of $X$ and $T_{2}$ reads the initial value of $Y$ in $\pi_{s} \cdot \rho \cdot \pi_{w} \cdot \pi_{f}$, and thus $T_{1}$ and $T_{2}$ cannot be both committed (at least one of the committed transactions must read the value written by the other) - a contradiction.

\subsection{Progressive TMs}

We move on to the stronger (than sequential TMs) class of progressive TMs. We introduce a new metric called protected data size that, intuitively, captures the number of t-objects that a transaction must exclusively control at some prefix of its execution. We first prove that any strict DAP progressive opaque TM must protect its entire write set at some point in its execution.

Secondly, we describe a constant stall, constant RAW/AWAR strict DAP opaque progressive TM that provides invisible reads and is implemented from read-write base objects. 


\subsubsection{A linear lower bound on the amount of protected data}

Let $M$ be a progressive TM implementation providing starvation-free TM-liveness. Intuitively, a t-object $X_{j}$ is protected at the end of some finite execution $\pi$ of $M$ if some transaction $T_{0}$ is about to atomically change the value of $X_{j}$ in its next step (e.g., by performing a compare-and-swap) or does not allow any concurrent transaction to read $X_{j}$ (e.g., by holding a "lock" on $X_{j}$ ).

Formally, let $\alpha \cdot \pi$ be an execution of $M$ such that $\pi$ is a t-sequential t-complete execution of a transaction $T_{0}$, where $W \operatorname{set}\left(T_{0}\right)=\left\{X_{1}, \ldots, X_{m}\right\}$. Let $u_{j}(j=1, \ldots, m)$ denote the value written by $T_{0}$ to t-object $X_{j}$ in $\pi$. In this section, let $\pi^{t}$ denote the $t$-th shortest prefix of $\pi$. Let $\pi^{0}$ denote the empty prefix.

For any $X_{j} \in W \operatorname{set}\left(T_{0}\right)$, let $T_{j}$ denote a transaction that tries to read $X_{j}$ and commit. Let $E_{j}^{t}=\alpha \cdot \pi^{t} \cdot \rho_{j}^{t}$ denote the extension of $\alpha \cdot \pi^{t}$ in which $T_{j}$ runs solo until it completes. Note that, since we only require the implementation to be starvation-free, $\rho_{j}^{t}$ can be infinite.

We say that $\alpha \cdot \pi^{t}$ is $(1, j)$-valent if the read operation performed by $T_{j}$ in $\alpha \cdot \pi^{t} \cdot \rho_{j}^{t}$ returns $u_{j}$ (the value written by $T_{0}$ to $\left.X_{j}\right)$. We say that $\alpha \cdot \pi^{t}$ is $(0, j)$-valent if the read operation performed by $T_{j}$ in $\alpha \cdot \pi^{t} \cdot \rho_{j}^{t}$ does not abort and returns an "old" value $u \neq u_{j}$. Otherwise, if the read operation of $T_{j}$ aborts or never returns in $\alpha \cdot \pi^{t} \cdot \rho_{j}^{t}$, we say that $\alpha \cdot \pi^{t}$ is $(\perp, j)$-valent.

Definition 4.1. We say that $T_{0}$ protects an object $X_{j}$ in $\alpha \cdot \pi^{t}$, where $\pi^{t}$ is the $t$-th shortest prefix of $\pi$ $(t>0)$ if one of the following conditions holds: (1) $\alpha \cdot \pi^{t}$ is $(0, j)$-valent and $\alpha \cdot \pi^{t+1}$ is $(1, j)$-valent, or (2) $\alpha \cdot \pi^{t}$ or $\alpha \cdot \pi^{t+1}$ is $(\perp, j)$-valent.

For strict disjoint-access parallel progressive TM, we show that every transaction running t-sequentially must protect every t-object in its write set at some point of its execution.

We observe that the no prefix of $\pi$ can be 0 and 1 -valent at the same time.

Lemma 4.5. There does not exist $\pi^{t}$, a prefix of $\pi$, and $i, j \in\{1, \ldots, m\}$ such that $\alpha \cdot \pi^{t}$ is both $(0, i)$-valent and $(1, j)$-valent.

Proof. By contradiction, suppose that there exist $i, j$ and $\alpha \cdot \pi^{t}$ that is both $(0, i)$-valent and $(1, j)$ valent. Since the implementation is strict DAP, there exists an execution of $M, E_{i j}^{t}=\alpha \cdot \pi^{t} \cdot \rho_{j}^{t} \cdot \rho_{i}^{t}$ that is indistinguishable to $T_{i}$ from $\alpha \cdot \pi^{t} \cdot \rho_{i}^{t}$. In $E_{i j}^{t}$, the only possible serialization is $T_{0}, T_{j}, T_{i}$. But $T_{i}$ returns the "old" value of $X_{i}$ and, thus, the serialization is not legal-a contradiction.

If $\alpha \cdot \pi^{t}$ is $(0, i)$-valent (resp., $(1, i)$-valent) for some $i$, we say that it is 0 -valent (resp., 1 -valent). By Lemma 4.5 the notions of 0 -valence and 1-valence are well-defined.

Theorem 4.6. Let $M$ be a progressive, opaque and strict disjoint-access-parallel TM implementation that provides starvation-free TM-liveness. Let $\alpha \cdot \pi$ be an execution of $M$, where $\pi$ is a t-sequential t-complete execution of a transaction $T_{0}$. Then, there exists $\pi^{t}$, a prefix of $\pi$, such that $T_{0}$ protects $\left|W \operatorname{set}\left(T_{0}\right)\right|$ t-objects in $\alpha \cdot \pi^{t}$.

Proof. Let sset $_{T_{0}}=\left\{X_{1}, \ldots, X_{m}\right\}$. Consider two cases:

(1) Suppose that $\pi$ has a prefix $\pi^{t}$ such that $\alpha \cdot \pi^{t}$ is 0 -valent and $\alpha \cdot \pi^{t+1}$ is 1 -valent. By Lemma 4.5 . there does not exists $i$, such that $\alpha \cdot \pi^{t}$ is $(1, i)$-valent and $\alpha \cdot \pi^{t+1}$ is $(0, i)$-valent. Thus, one of the following are true

- For every $i \in\{1, \ldots, m\}, \alpha \cdot \pi^{t}$ is $(0, i)$-valent and $\alpha \cdot \pi^{t+1}$ is $(1, i)$-valent

- At least one of $\alpha \cdot \pi^{t}$ and $\alpha \cdot \pi^{t+1}$ is $(\perp, i)$-valent, i.e., the t-operation of $T_{i}$ aborts or never returns

In either case, $T_{0}$ protects $m$ t-objects in $\alpha \cdot \pi^{t}$. 
(2) Now suppose that such $\pi^{t}$ does not exists, i.e., there is no $i \in\{1, \ldots, m\}$ and $t \in\{0,|\pi|-1\}$ such that $E_{i}^{t}$ exists and returns an old value, and $E_{i}^{t+1}$ exists and returns a new value.

Suppose there exists $s, t, 0<s+1<t, S \subseteq\{1, \ldots, m\}$, such that:

- $\alpha \cdot \pi^{s}$ is 0 -valent,

- $\alpha \cdot \pi^{t}$ is 1 -valent,

- for all $r, s<r<t$, and for all $i \in S, \alpha \cdot \pi^{r}$ is $(\perp, i)$-valent.

We say that $s+1, \ldots, t-1$ is a protecting fragment for t-objects $\left\{X_{j} \mid j \in S\right\}$.

Since $M$ is opaque and progressive, $\alpha \cdot \pi^{0}=\alpha$ is 0 -valent and $\alpha \cdot \pi$ is 1 -valent. Thus, the assumption of Case (2) implies that for each $X_{i}$, there exists a protecting fragment for $\left\{X_{i}\right\}$. In particular, there exists a protecting fragment for $\left\{X_{1}\right\}$.

Now we proceed by induction. Let $\pi_{s+1}, \ldots, \pi_{t-1}$ be a protecting fragment for $\left\{X_{1}, \ldots, X_{u-1}\right\}$ such that $u \leq m$.

Now we claim that there must be a subfragment of $s+1, \ldots, t-1$ that protects $\left\{X_{1}, \ldots, X_{u}\right\}$.

Suppose not. Thus, there exists $r, s<r<t$, such that $\alpha \cdot \pi^{r}$ is $(0, u)$-valent or $(1, u)$-valent. Suppose first that $\alpha \cdot \pi^{r}$ is $(1, u)$-valent. Since $\alpha \cdot \pi^{s}$ is $(0, i)$-valent for some $i \neq u$, by Lemma 4.5 and the assumption of Case (2), there must exist $s^{\prime}, t^{\prime}, s<s^{\prime}+1<t^{\prime} \leq r$ such that

- $\alpha \cdot \pi^{s^{\prime}}$ is 0 -valent,

- $\alpha \cdot \pi^{t^{\prime}}$ is 1 -valent,

- for all $r^{\prime}, s^{\prime}<r^{\prime}<t^{\prime}, \alpha \cdot \pi^{r^{\prime}}$ is $(\perp, u)$-valent.

As a result, $s^{\prime}+1, \ldots, t^{\prime}-1$ is a protecting fragment for $\left\{X_{1}, \ldots, X_{u}\right\}$. The case when $\alpha \cdot \pi^{r}$ is $(0, u)$-valent is symmetric, except that now we should consider fragment $r, \ldots, t$ instead of $s, \ldots, r$.

Thus, there exists a subfragment of $s+1, \ldots, t-1$ that protects $\left\{X_{1}, \ldots, X_{u}\right\}$. By induction, we obtain a protecting fragment $s^{\prime \prime}+1, \ldots, t^{\prime \prime}-1$ for $\left\{X_{1}, \ldots, X_{m}\right\}$. Thus, any prefix $\alpha \cdot \pi^{r}$, where $s^{\prime \prime}<r<t^{\prime \prime}$ protects exactly $m$ t-objects.

In both cases, there is a prefix of $\alpha \cdot \pi$ that protects exactly $m$ t-objects.

The lower bound of Theorem 4.6 is tight: it is matched by all progressive implementations we are aware of, including Algorithm 4.1 described in the next section.

\subsubsection{A constant stall and constant expensive synchronization strict DAP opaque TM}

In this section, we describe a cheap progressive, opaque TM implementation $L P$ (Algorithm 4.1). Our TM $L P$, every transaction performs at most a single RAW, every t-read operation incurs $O(1)$ memory stalls and maintains exactly one version of every t-object at any prefix of an execution. Moreover, the implementation is strict DAP and uses only read-write base objects.

Base objects. For every t-object $X_{j}, L P$ maintains a base object $v_{j}$ that stores the value of $X_{j}$. Additionally, for each $X_{j}$, we maintain a bit $L_{j}$, which if set, indicates the presence of an updating transaction writing to $X_{j}$. Also, for every process $p_{i}$ and t-object $X_{j}, L P$ maintains a single-writer bit $r_{i j}$ to which only $p_{i}$ is allowed to write. Each of these base objects may be accessed only via read and write primitives.

Read operations. The implementation first reads the value of t-object $X_{j}$ from base object $v_{j}$ and then reads the bit $L_{j}$ to detect contention with an updating transaction. If $L_{j}$ is set, the transaction is aborted; if not, read validation is performed on the entire read set. If the validation fails, the transaction 


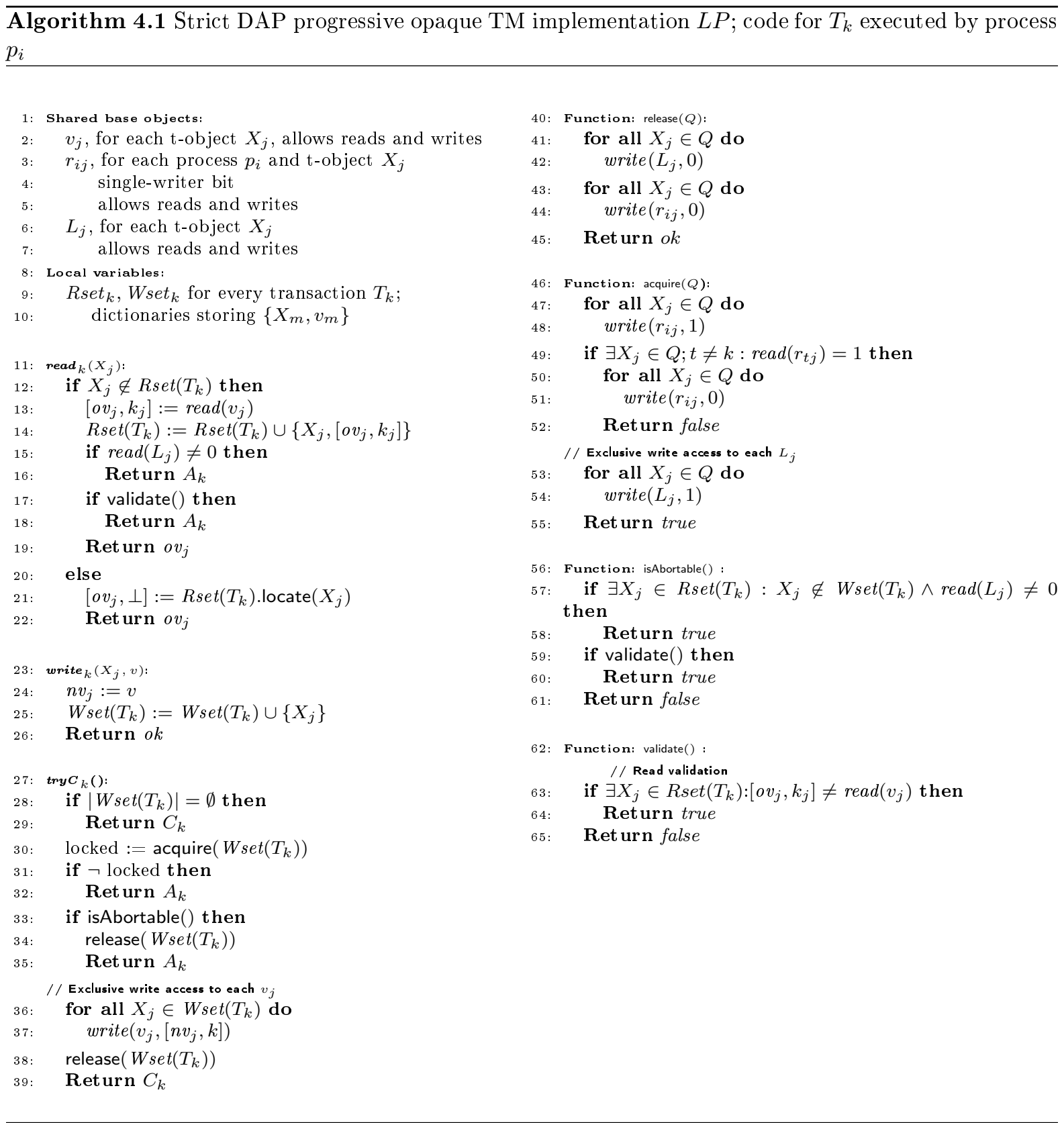

is aborted. Otherwise, the implementation returns the value of $X_{j}$. For a read-only transaction $T_{k}$, $\operatorname{try} C_{k}$ simply returns the commit response.

Updating transactions. The write $_{k}(X, v)$ implementation by process $p_{i}$ simply stores the value $v$ locally, deferring the actual updates to $\operatorname{try} C_{k}$. During $\operatorname{try} C_{k}$, process $p_{i}$ attempts to obtain exclusive write access to every $X_{j} \in W \operatorname{set}\left(T_{k}\right)$. This is realized through the single-writer bits, which ensure that no other transaction may write to base objects $v_{j}$ and $L_{j}$ until $T_{k}$ relinquishes its exclusive write access to $W \operatorname{set}\left(T_{k}\right)$. Specifically, process $p_{i}$ writes 1 to each $r_{i j}$, then checks that no other process $p_{t}$ has written 1 to any $r_{t j}$ by executing a series of reads (incurring a single RAW). If there exists such a process that concurrently contends on write set of $T_{k}$, for each $X_{j} \in W \operatorname{set}\left(T_{k}\right), p_{i}$ writes 0 to $r_{i j}$ and aborts $T_{k}$. If successful in obtaining exclusive write access to $W \operatorname{set}\left(T_{k}\right), p_{i}$ sets the bit $L_{j}$ for each $X_{j}$ in its write set. Implementation of $\operatorname{try} C_{k}$ now checks if any t-object in its read set is concurrently contended by another transaction and then validates its read set. If there is contention on the read set or validation fails (indicating the presence of a conflicting transaction), the transaction is aborted. If not, $p_{i}$ writes the 
values of the t-objects to shared memory and relinquishes exclusive write access to each $X_{j} \in W \operatorname{set}\left(T_{k}\right)$ by writing 0 to each of the base objects $L_{j}$ and $r_{i j}$.

Complexity. Read-only transactions do not apply any nontrivial primitives. Any updating transaction performs at most a single RAW in the course of acquiring exclusive write access to the transaction's write set. Thus, every transaction performs $O(1)$ non-overlapping RAWs in any execution.

Recall that a transaction may write to base objects $v_{j}$ and $L_{j}$ only after obtaining exclusive write access to t-object $X_{j}$, which in turn is realized via single-writer base objects. Thus, no transaction performs a write to any base object $b$ immediately after a write to $b$ by another transaction, i.e., every transaction incurs only $O(1)$ memory stalls on account of any event it performs. The read $d_{k}\left(X_{j}\right)$ implementation reads base objects $v_{j}$ and $L_{j}$, followed by the validation phase in which it reads $v_{k}$ for each $X_{k}$ in its current read set. Note that if the first read in the validation phase incurs a stall, then $\operatorname{read}_{k}\left(X_{j}\right)$ aborts. It follows that each t-read incurs $O(1)$ stalls in every execution.

Proof of opacity. We now prove that $L P$ implements an opaque TM.

We introduce the following technical definition: process $p_{i}$ holds a lock on $X_{j}$ after an execution $\pi$ of Algorithm 4.1 if $\pi$ contains the invocation of acquire $(Q), X_{j} \in Q$ by $p_{i}$ that returned true, but does not contain a subsequent invocation of release $\left(Q^{\prime}\right), X_{j} \in Q^{\prime}$, by $p_{i}$ in $\pi$.

Lemma 4.7. For any object $X_{j}$, and any execution $\pi$ of Algorithm 4.1. there exists at most one process that holds a lock on $X_{j}$ after $\pi$.

Proof. Assume, by contradiction, that there exists an execution $\pi$ after which processes $p_{i}$ and $p_{k}$ hold a lock on the same object, say $X_{j}$. In order to hold the lock on $X_{j}$, process $p_{i}$ writes 1 to register $r_{i j}$ and then checks if any other process $p_{k}$ has written 1 to $r_{k j}$. Since the corresponding operation acquire $(Q)$, $X_{j} \in Q$ invoked by $p_{i}$ returns true, $p_{i}$ read 0 in $r_{k j}$ in Line 49. But then $p_{k}$ also writes 1 to $r_{k j}$ and later reads that $r_{i j}$ is 1 . This is because $p_{k}$ can write 1 to $r_{k j}$ only after the read of $r_{k j}$ returned 0 to $p_{i}$ which is preceded by the write of 1 to $r_{i j}$. Hence, there exists an object $X_{j}$ such that $r_{i j}=1 ; i \neq k$, but the conditional in Line 49 returns true to process $p_{k}-$ a contradiction.

Observation 4.8. Let $\pi$ be any execution of Algorithm 4.1. Then, any updating transaction $T_{k} \in \operatorname{txns}(\pi)$ executed by process $p_{i}$ writes to base object $v_{j}$ (in Line 37) for some $X_{j} \in W \operatorname{wet}\left(T_{k}\right)$ immediately after $\pi$ iff $p_{i}$ holds the lock on $X_{j}$ after $\pi$.

Lemma 4.9. Algorithm 4.1 implements an opaque TM.

Proof. Let $E$ by any finite execution of Algorithm 4.1. Let $<_{E}$ denote a total-order on events in $E$.

Let $H$ denote a subsequence of $E$ constructed by selecting linearization points of t-operations performed in $E$. The linearization point of a t-operation $o p$, denoted as $\ell_{o p}$ is associated with a base object event or an event performed between the invocation and response of $o p$ using the following procedure.

Completions. First, we obtain a completion of $E$ by removing some pending invocations and adding responses to the remaining pending invocations involving a transaction $T_{k}$ as follows: every incomplete read $_{k}$, write $e_{k}$ operation is removed from $E$; an incomplete $\operatorname{try} C_{k}$ is removed from $E$ if $T_{k}$ has not performed any write to a base object during the release function in Line 38, otherwise it is completed by including $C_{k}$ after $E$.

Linearization points. Now a linearization $H$ of $E$ is obtained by associating linearization points to t-operations in the obtained completion of $E$ as follows:

- For every t-read $o p_{k}$ that returns a non- $\mathrm{A}_{k}$ value, $\ell_{o p_{k}}$ is chosen as the event in Line 13 of Algorithm 4.1. else, $\ell_{o p_{k}}$ is chosen as invocation event of $o p_{k}$

- For every $o p_{k}=$ write $_{k}$ that returns, $\ell_{o p_{k}}$ is chosen as the invocation event of $o p_{k}$

- For every $o p_{k}=\operatorname{try} C_{k}$ that returns $C_{k}$ such that $W \operatorname{set}\left(T_{k}\right) \neq \emptyset, \ell_{o p_{k}}$ is associated with the response of acquire in Line 30, else if $o p_{k}$ returns $A_{k}, \ell_{o p_{k}}$ is associated with the invocation event of $o p_{k}$ 
- For every $o p_{k}=\operatorname{try} C_{k}$ that returns $C_{k}$ such that $W \operatorname{set}\left(T_{k}\right)=\emptyset, \ell_{o p_{k}}$ is associated with Line 29 $<_{H}$ denotes a total-order on t-operations in the complete sequential history $H$.

Serialization points. The serialization of a transaction $T_{j}$, denoted as $\delta_{T_{j}}$ is associated with the linearization point of a t-operation performed within the execution of $T_{j}$.

We obtain a t-complete history $\bar{H}$ from $H$ as follows: for every transaction $T_{k}$ in $H$ that is complete, but not t-complete, we insert $\operatorname{try} C_{k} \cdot A_{k}$ after $H$.

A t-complete t-sequential history $S$ is obtained by associating serialization points to transactions in $\bar{H}$ as follows:

- If $T_{k}$ is an update transaction that commits, then $\delta_{T_{k}}$ is $\ell_{t r y C_{k}}$

- If $T_{k}$ is a read-only or aborted transaction in $\bar{H}, \delta_{T_{k}}$ is assigned to the linearization point of the last t-read that returned a non- $\mathrm{A}_{k}$ value in $T_{k}$

$<_{S}$ denotes a total-order on transactions in the t-sequential history $S$.

Claim 4.10. If $T_{i} \prec_{H} T_{j}$, then $T_{i}<_{S} T_{j}$

Proof. This follows from the fact that for a given transaction, its serialization point is chosen between the first and last event of the transaction implying if $T_{i} \prec_{H} T_{j}$, then $\delta_{T_{i}}<_{E} \delta_{T_{j}}$ implies $T_{i}<_{S} T_{j}$.

Claim 4.11. Let $T_{k}$ be any updating transaction that returns false from the invocation of is Abortable in Line 33 . Then, $T_{k}$ returns $C_{k}$ within a finite number of its own steps in any extension of $E$.

Proof. Observer that $T_{k}$ performs the write to base objects $v_{j}$ for every $X_{j} \in W \operatorname{set}\left(T_{k}\right)$ and then invokes release in Lines 37 and 38 respectively. Since neither of these involve aborting the transaction or contain unbounded loops or waiting statements, it follows that $T_{k}$ will return $C_{k}$ within a finite number of its steps.

Claim 4.12. $S$ is legal.

Proof. Observe that for every $\operatorname{read}_{j}\left(X_{m}\right) \rightarrow v$, there exists some transaction $T_{i}$ that performs write $_{i}\left(X_{m}, v\right)$ and completes the event in Line 37 such that $\operatorname{read}_{j}\left(X_{m}\right) \nprec_{H}^{R T}$ write $_{i}\left(X_{m}, v\right)$. More specifically, $\operatorname{read}_{j}\left(X_{m}\right)$ returns as a non-abort response, the value of the base object $v_{m}$ and $v_{m}$ can be updated only by a transaction $T_{i}$ such that $X_{m} \in W \operatorname{Set}\left(T_{i}\right)$. Since $\operatorname{read}_{j}\left(X_{m}\right)$ returns the response $v$, the event in Line 13 succeeds the event in Line 37 performed by $\operatorname{try} C_{i}$. Consequently, by Claim 4.11 and the assignment of linearization points, $\ell_{t r y C_{i}}<_{E} \ell_{\text {read }_{j}\left(X_{m}\right)}$. Since, for any updating committing transaction $T_{i}, \delta_{T_{i}}=\ell_{\text {try }} C_{i}$, by the assignment of serialization points, it follows that $\delta_{T_{i}}<_{E} \delta_{T_{j}}$.

Thus, to prove that $S$ is legal, it suffices to show that there does not exist a transaction $T_{k}$ that returns $C_{k}$ in $S$ and performs write $_{k}\left(X_{m}, v^{\prime}\right) ; v^{\prime} \neq v$ such that $T_{i}<_{S} T_{k}<_{S} T_{j}$. Suppose that there exists a committed transaction $T_{k}, X_{m} \in W \operatorname{set}\left(T_{k}\right)$ such that $T_{i}<_{S} T_{k}<_{S} T_{j}$.

$T_{i}$ and $T_{k}$ are both updating transactions that commit. Thus,

$$
\begin{gathered}
\left(T_{i}<_{S} T_{k}\right) \Longleftrightarrow\left(\delta_{T_{i}}<_{E} \delta_{T_{k}}\right) \\
\left(\delta_{T_{i}}<_{E} \delta_{T_{k}}\right) \Longleftrightarrow\left(\ell_{t r y C_{i}}<_{E} \ell_{t r y C_{k}}\right)
\end{gathered}
$$

Since, $T_{j}$ reads the value of $X$ written by $T_{i}$, one of the following is true: $\ell_{\text {try } C_{i}}<_{E} \ell_{\text {try }_{k}}<_{E} \ell_{\text {read }}\left(X_{m}\right)$ or $\ell_{t r y C_{i}}<_{E} \ell_{\text {read }_{j}\left(X_{m}\right)}<_{E} \ell_{t r y C_{k}}$. Let $T_{i}$ and $T_{k}$ be executed by processes $p_{i}$ and $p_{k}$ respectively.

Consider the case that $\ell_{\operatorname{try} C_{i}}<_{E} \ell_{\text {try } C_{k}}<_{E} \ell_{\text {read }_{j}\left(X_{m}\right)}$.

By the assignment of linearization points, $T_{k}$ returns a response from the event in Line 30 before the read of $v_{m}$ by $T_{j}$ in Line 13 . Since $T_{i}$ and $T_{k}$ are both committed in $E, p_{k}$ returns true from the event in Line 30 only after $T_{i}$ writes 0 to $r_{i m}$ in Line 44 (Lemma 4.17). 
Recall that $\operatorname{read}_{j}\left(X_{m}\right)$ checks if $X_{m}$ is locked by a concurrent transaction (i.e $L_{j} \neq 0$ ), then performs read-validation (Line 15) before returning a matching response. Consider the following possible sequence of events: $T_{k}$ returns true from the acquire function invocation, sets $L_{j}$ to 1 for every $X_{j} \in W \operatorname{set}\left(T_{k}\right)$ (Line 54 and updates the value of $X_{m}$ to shared-memory (Line 37). The implementation of read $d_{j}\left(X_{m}\right)$ then reads the base object $v_{m}$ associated with $X_{m}$ after which $T_{k}$ releases $X_{m}$ by writing 0 to $r_{k m}$ and finally $T_{j}$ performs the check in Line 15. However, $\operatorname{read}_{j}\left(X_{m}\right)$ is forced to return $A_{j}$ because $X_{m} \in \operatorname{Rset}\left(T_{j}\right)$ (Line 14 and has been invalidated since last reading its value. Otherwise suppose that $T_{k}$ acquires exclusive access to $X_{m}$ by writing 1 to $r_{k m}$ and returns true from the invocation of acquire, updates $v_{m}$ in Line 37), $T_{j}$ reads $v_{m}, T_{j}$ performs the check in Line 15 and finally $T_{k}$ releases $X_{m}$ by writing 0 to $r_{k m}$. Again, $\operatorname{read}_{j}\left(X_{m}\right)$ returns $A_{j}$ since $T_{j}$ reads that $r_{k m}$ is 1 - contradiction.

Thus, $\ell_{\text {try }_{i}}<_{E} \ell_{\text {read }_{j}(X)}<_{E} \ell_{t r y C_{k}}$.

We now need to prove that $\delta_{T_{j}}$ indeed precedes $\ell_{t r y C_{k}}$ in $E$.

Consider the two possible cases:

- Suppose that $T_{j}$ is a read-only or aborted transaction in $\bar{H}$. Then, $\delta_{T_{j}}$ is assigned to the last t-read performed by $T_{j}$ that returns a non- $\mathrm{A}_{j}$ value. If $\operatorname{read}_{j}\left(X_{m}\right)$ is not the last t-read performed by $T_{j}$ that returned a non- $\mathrm{A}_{j}$ value, then there exists a $\operatorname{read}_{j}\left(X_{z}\right)$ performed by $T_{j}$ such that $\ell_{\text {read }_{j}\left(X_{m}\right)}<_{E} \ell_{\text {try } C_{k}}<_{E} \ell_{\text {read }_{j}\left(X_{z}\right)}$. Now assume that $\ell_{t r y C_{k}}$ must precede $\ell_{\text {read }_{j}\left(X_{z}\right)}$ to obtain a legal $S$. Since $T_{k}$ and $T_{j}$ are concurrent in $E$, we are restricted to the case that $T_{k}$ performs a write $_{k}\left(X_{z}, v\right)$ and $\operatorname{read}_{j}\left(X_{z}\right)$ returns $v$. However, we claim that this t-read of $X_{z}$ must abort by performing the checks in Line 15 . Observe that $T_{k}$ writes 1 to $L_{m}, L_{z}$ each (Line 54) and then writes new values to base objects $v_{m}, v_{z}$ (Line 37 ). Since $\operatorname{read}_{j}\left(X_{z}\right)$ returns a non- $A_{j}$ response, $T_{k}$ writes 0 to $L_{z}$ before the read of $L_{z}$ by $\operatorname{read}_{j}\left(\bar{X}_{z}\right)$ in Line 15 . Thus, the t-read of $X_{z}$ would return $A_{j}$ (in Line 17 after validation of the read set since $X_{m}$ has been updated - contradiction to the assumption that it the last t-read by $T_{j}$ to return a non- $A_{j}$ response.

- Suppose that $T_{j}$ is an updating transaction that commits, then $\delta_{T_{j}}=\ell_{t r y C_{j}}$ which implies that $\ell_{\text {read }_{j}\left(X_{m}\right)}<_{E} \ell_{t r y C_{k}}<_{E} \ell_{t r y C_{j}}$. Then, $T_{j}$ must necessarily perform the checks in Line 33 and read that $L_{m}$ is 1 . Thus, $T_{j}$ must return $A_{j}$-contradiction to the assumption that $T_{j}$ is a committed transaction.

The conjunction of Claims 4.10 and 4.12 establish that Algorithm 4.1 is opaque.

We can now prove the following theorem:

Theorem 4.13. Algorithm 4.1 describes a progressive, opaque and strict DAP TM implementation LP that provides wait-free TM-liveness, uses invisible reads, uses only read-write base objects, and for every execution $E$ and transaction $T_{k} \in \operatorname{txns}(E)$ :

- $T_{k}$ performs at most a single $R A W$, and

- every t-read operation invoked by $T_{k}$ incurs $O(1)$ memory stalls in $E$, and

- every complete t-read operation invoked by $T_{k}$ performs $O\left(\left|R \operatorname{set}\left(T_{k}\right)\right|\right)$ steps in E.

Proof. (TM-liveness and TM-progress) Since none of the implementations of the t-operations in Algorithm 4.1 contain unbounded loops or waiting statements, every t-operation ${ }_{0} p_{k}$ returns a matching response after taking a finite number of steps in every execution. Thus, Algorithm 4.1 provides wait-free TM-liveness.

To prove progressiveness, we proceed by enumerating the cases under which a transaction $T_{k}$ may be aborted. 
- Suppose that there exists a $\operatorname{read}_{k}\left(X_{j}\right)$ performed by $T_{k}$ that returns $A_{k}$ from Line 15 . Thus, there exists a process $p_{t}$ executing a transaction that has written 1 to $r_{t j}$ in Line 48 but has not yet written 0 to $r_{t j}$ in Line 44 or some t-object in $\operatorname{Rset}\left(T_{k}\right)$ has been updated since its t-read by $T_{k}$. In both cases, there exists a concurrent transaction performing a t-write to some t-object in $R \operatorname{set}\left(T_{k}\right)$.

- Suppose that $\operatorname{try} C_{k}$ performed by $T_{k}$ that returns $A_{k}$ from Line 31 . Thus, there exists a process $p_{t}$ executing a transaction that has written 1 to $r_{t j}$ in Line 48 but has not yet written 0 to $r_{t j}$ in Line 44. Thus, $T_{k}$ encounters step-contention with another transaction that concurrently attempts to update a t-object in $W \operatorname{set}\left(T_{k}\right)$.

- Suppose that $\operatorname{try} C_{k}$ performed by $T_{k}$ that returns $A_{k}$ from Line 33 . Since $T_{k}$ returns $A_{k}$ from Line 33 for the same reason it returns $A_{k}$ after Line 15 the proof follows.

(Strict disjoint-access parallelism) Consider any execution $E$ of Algorithm 4.1 and let $T_{i}$ and $T_{j}$ be any two transactions that participate in $E$ and access the same base object $b$ in $E$.

- Suppose that $T_{i}$ and $T_{j}$ contend on base object $v_{j}$ or $L_{j}$. Since for every t-object $X_{j}$, there exists distinct base objects $v_{j}$ and $L_{j}, T_{j}$ and $T_{j}$ contend on $v_{j}$ only if $X_{j} \in \operatorname{Dset}\left(T_{i}\right) \cap \operatorname{Dset}\left(T_{j}\right)$.

- Suppose that $T_{i}$ and $T_{j}$ contend on base object $r_{i j}$. Without loss of generality, let $p_{i}$ be the process executing transaction $T_{i} ; X_{j} \in W \operatorname{set}\left(T_{i}\right)$ that writes 1 to $r_{i j}$ in Line 48 . Indeed, no other process executing a transaction that writes to $X_{j}$ can write to $r_{i j}$. Transaction $T_{j}$ reads $r_{i j}$ only if $X_{j} \in \operatorname{Dset}\left(T_{j}\right)$ as evident from the accesses performed in Lines $48,49,44,27$.

Thus, $T_{i}$ and $T_{j}$ access the same base object only if they access a common t-object.

(Opacity) Follows from Lemma 4.9 .

(Invisible reads) Observe that read-only transactions do not perform any nontrivial events. Secondly, in any execution $E$ of Algorithm 4.1, and any transaction $T_{k} \in \operatorname{txns}(E)$, if $X_{j} \in \operatorname{Rset}\left(T_{k}\right), T_{k}$ does not write to any of the base objects associated with $X_{j}$ nor write any information that reveals its read set to other transactions.

(Complexity) Consider any execution $E$ of Algorithm 4.1

- For any $T_{k} \in \operatorname{txns}(E)$, each $\operatorname{read}_{k}$ only applies trivial primitives in $E$ while $\operatorname{try} C_{k}$ simply returns $C_{k}$ if $W \operatorname{set}\left(T_{k}\right)=\emptyset$. Thus, Algorithm 4.1 uses invisible reads.

- Any read-only transaction $T_{k} \in \operatorname{txns}(E)$ not perform any RAW or AWAR. An updating transaction $T_{k}$ executed by process $p_{i}$ performs a sequence of writes (Line 48 to base objects $\left\{r_{i j}\right\}: X_{j} \in$ $W \operatorname{set}\left(T_{k}\right)$, followed by a sequence of reads to base objects $\left\{r_{t j}\right\}: t \in\{1, \ldots, n\}, X_{j} \in W \operatorname{set}\left(T_{k}\right)$ (Line 49 thus incurring a single multi-RAW.

- Let $e$ be a write event performed by some transaction $T_{k}$ executed by process $p_{i}$ in $E$ on base objects $v_{j}$ and $L_{j}$ (Lines 37 and 54 ). Any transaction $T_{k}$ performs a write to $v_{j}$ or $L_{j}$ only after $T_{k}$ writes 0 to $r_{i j}$, for every $X_{j} \in W \operatorname{set}\left(T_{k}\right)$. Thus, by Lemmata 4.17 and 4.9 it follows that events that involve an access to either of these base objects incurs $O(1)$ stalls.

Let $e$ be a write event on base object $r_{i j}$ (Line 48 while writing to t-object $X_{j}$. By Algorithm 4.1. no other process can write to $r_{i j}$. It follows that any transaction $T_{k} \in \operatorname{txns}(E)$ incurs $O(1)$ memory stalls on account of any event it performs in $E$. Observe that any t-read read $d_{k}\left(X_{j}\right)$ only accesses base objects $v_{j}, L_{j}$ and other value base objects in $R s e t\left(T_{k}\right)$. But as already established above, these are $O(1)$ stall events. Hence, every t-read operation incurs $O(1)$-stalls in $E$.

The following corollary follows from Theorems 4.13 and 4.2 .

Corollary 4.14. Let $M$ be any weak DAP progressive opaque TM implementation providing ICF TMliveness and weak invisible reads. Then, for every execution $E$ and each read-only transaction $T_{k} \in$ txns $(E), T_{k}$ performs $\Theta\left(m^{2}\right)$ steps in $E$, where $m=\left|\operatorname{Rset}_{E}\left(T_{k}\right)\right|$. 


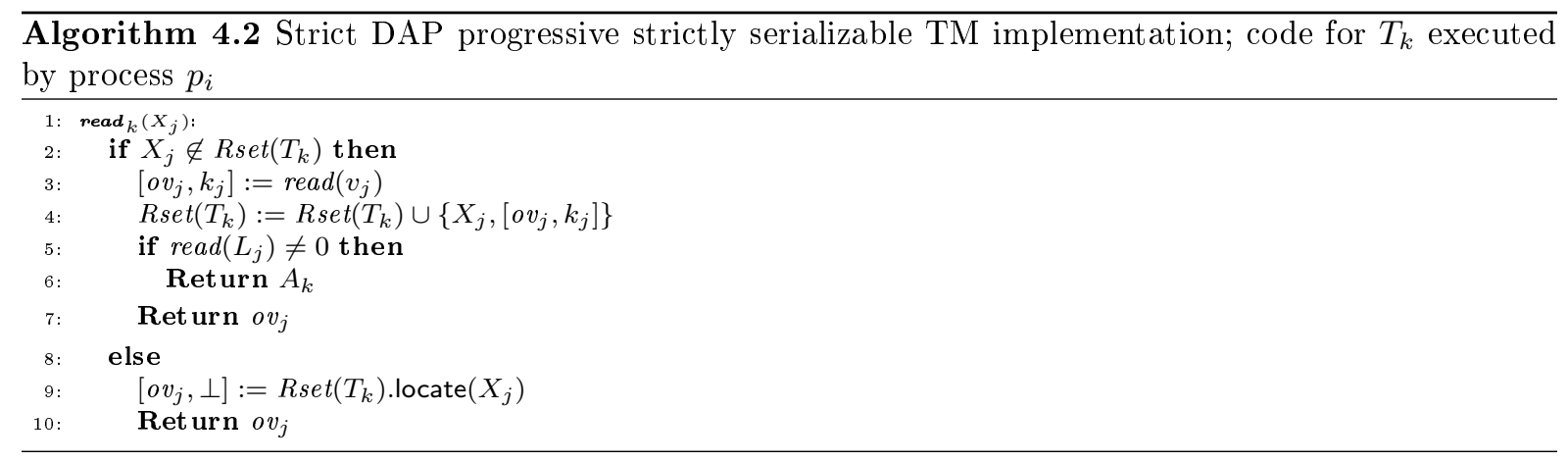

Similarly, we can prove an almost matching upper bound for Theorem 4.2 for strictly serializable progressive TMs.

Consider Algorithm 4.2 that is a simplification of the opaque progressive TM in Algorithm 4.1. we remove the validation performed in the implementation of a t-read, i.e., Line 17 in Algorithm 4.1; otherwise, the two algorithms are identical. It is easy to see this results in a strictly serializable (but not opaque) TM implementation. Thus,

Theorem 4.15. Algorithm 4.2 describes a progressive, strictly serializable and strict DAP TM implementation that provides wait-free TM-liveness, uses invisible reads, uses only read-write base objects, and for every execution $E$ and transaction $T_{k} \in \operatorname{txns}(E)$ : every $t$-read operation invoked by $T_{k}$ performs $O(1)$ steps and try $C_{k}$ performs $O\left(\left|R \operatorname{set}\left(T_{k}\right)\right|\right)$ steps in $E$.

Corollary 4.16. Let $M$ be any weak DAP progressive strictly serializable TM implementation providing ICF TM-liveness and weak invisible reads. Then, for every execution $E$ and each read-only trans-

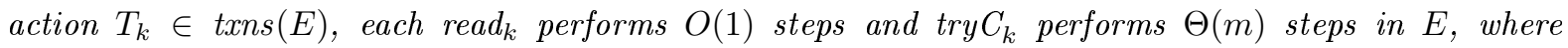
$m=\left|\operatorname{Rset}_{E}\left(T_{k}\right)\right|$.

\subsection{Strongly progressive TMs}

In this section, we prove that every strongly progressive strictly serializable TM that uses only read, write and conditional primitives has an execution in which in which $n$ concurrent processes perform transactions on a single data item and incur $\Omega(\log n)$ remote memory references [13].

We then describe a constant RAW/AWAR strongly progressive TM providing starvation-free TM-liveness from read-write base objects.

\subsubsection{A $\Omega(n \log n)$ lower bound on remote memory references}

Our lower bound on RMR complexity of strongly progressive TMs is derived by reduction to mutual exclusion.

Mutual exclusion. The mutex object supports two operations: Entry and Exit, both of which return the response $o k$. We say that a process $p_{i}$ is in the critical section after an execution $\pi$ if $\pi$ contains the invocation of Entry by $p_{i}$ that returns $o k$, but does not contain a subsequent invocation of Exit by $p_{i}$ in $\pi$.

A mutual exclusion implementation satisfies the following properties:

- (Mutual-exclusion) After any execution $\pi$, there exists at most one process that is in the critical section. 
- (Deadlock-freedom) Let $\pi$ be any execution that contains the invocation of Enter by process $p_{i}$. Then, in every extension of $\pi$ in which every process takes infinitely many steps, some process is in the critical section.

- (Finite-exit) Every process completes the Exit operation within a finite number of steps.

We describe an implementation of a mutex object $L(M)$ from a strictly serializable, strongly progressive TM implementation $M$ providing wait-free TM-liveness (Algorithm 4.3). The algorithm is based on the mutex implementation in [82].

Given a sequential implementation, we use a TM to execute the sequential code in a concurrent environment by encapsulating each sequential operation within an atomic transaction that replaces each read and write of a t-object with the transactional read and write implementations, respectively. If the transaction commits, then the result of the operation is returned; otherwise if one of the transactional operations aborts. For instance, in Algorithm 4.3. we wish to atomically read a t-object $X$, write a new value to it and return the old value of $X$ prior to this write. To achieve this, we employ a strictly serializable TM implementation $M$. Since we assume that $M$ is strongly progressive, in every execution, at least one transaction successfully commits and the value of $X$ is returned.

Shared objects. We associate each process $p_{i}$ with two alternating identities $\left[p_{i}\right.$, face $\left._{i}\right] ;$ face $_{i} \in\{0,1\}$. The strongly progressive TM implementation $M$ is used to enqueue processes that attempt to enter the critical section within a single t-object $X$ (initially $\perp$ ). For each $\left[p_{i}\right.$, face $\left._{i}\right], L(M)$ uses a register bit Done $\left[p_{i}\right.$, face $\left._{i}\right]$ that indicates if this face of the process has left the critical section or is executing the Entry operation. Additionally, we use a register $S u c c\left[p_{i}\right.$, face $\left._{i}\right]$ that stores the process expected to succeed $p_{i}$ in the critical section. If $S u c c\left[p_{i}\right.$, face $\left._{i}\right]=p_{j}$, we say that $p_{j}$ is the successor of $p_{i}$ (and $p_{i}$ is the predecessor of $p_{j}$ ). Intuitively, this means that $p_{j}$ is expected to enter the critical section immediately after $p_{i}$. Finally, $L(M)$ uses a 2-dimensional bit array Lock: for each process $p_{i}$, there are $n-1$ registers associated with the other processes. For all $j \in\{0, \ldots, n-1\} \backslash\{i\}$, the registers $\operatorname{Lock}\left[p_{i}\right]\left[p_{j}\right]$ are local to $p_{i}$ and registers $\operatorname{Lock}\left[p_{j}\right]\left[p_{i}\right]$ are remote to $p_{i}$. Process $p_{i}$ can only access registers in the Lock array that are local or remote to it.

Entry operation. A process $p_{i}$ adopts a new identity face $i_{i}$ and writes false to Done $\left(p_{i}\right.$, face $\left._{i}\right)$ to indicate that $p_{i}$ has started the Entry operation. Process $p_{i}$ now initializes the successor of $\left[p_{i}\right.$, face $\left.e_{i}\right]$ by writing $\perp$ to $S u c c\left[p_{i}\right.$, face $\left._{i}\right]$. Now, $p_{i}$ uses a strongly progressive TM implementation $M$ to atomically store its pid and identity i.e., face ${ }_{i}$ to t-object $X$ and returns the pid and identity of its predecessor, say $\left[p_{j}\right.$, face $\left._{j}\right]$. Intuitively, this suggests that $\left[p_{i}\right.$, face $\left._{i}\right]$ is scheduled to enter the critical section immediately after $] p_{j}$, face $_{j}$ ] exits the critical section. Note that if $p_{i}$ reads the initial value of t-object $X$, then it immediately enters the critical section. Otherwise it writes locked to the register Lock $\left[p_{i}, p_{j}\right]$ and sets itself to be the successor of $\left[p_{j}\right.$, face $\left._{j}\right]$ by writing $p_{i}$ to $S u c c\left[p_{j}\right.$, face $\left.j\right]$. Process $p_{i}$ now checks if $p_{j}$ has started the Exit operation by checking if Done $\left[p_{j}\right.$, face $\left._{j}\right]$ is set. If it is, $p_{i}$ enters the critical section; otherwise $p_{i}$ spins on the register $\operatorname{Lock}\left[p_{i}\right]\left[p_{j}\right]$ until it is unlocked.

Exit operation. Process $p_{i}$ first indicates that it has exited the critical section by setting Done $\left[p_{i}\right.$, face $\left._{i}\right]$, following which it unlocks the register $\operatorname{Lock}\left[\operatorname{Succ}\left[p_{i}\right.\right.$, face $\left.\left._{i}\right]\right]\left[p_{i}\right]$ to allow $p_{i}$ 's successor to enter the critical section.

Lemma 4.17. The implementation $L(M)$ (Algorithm 4.3) satisfies mutual exclusion.

Proof. Let $E$ be any execution of $L(M)$. We say that $\left[p_{i}\right.$, face $\left._{i}\right]$ is the successor of $\left[p_{j}\right.$, face $\left._{j}\right]$ if $p_{i}$ reads the value of prev in Line 25 to be $\left[p_{j}\right.$, face $\left._{j}\right]$ (and $\left[p_{j}\right.$, face $\left._{j}\right]$ is the predecessor of $\left[p_{i}\right.$, face $\left._{i}\right]$ ); otherwise if $p_{i}$ reads the value to be $\perp$, we say that $p_{i}$ has no predecessor.

Suppose by contradiction that there exist processes $p_{i}$ and $p_{j}$ that are both inside the critical section after $E$. Since $p_{i}$ is inside the critical section, either (1) $p_{i}$ read prev $=\perp$ in Line 23 or $(2) p_{i}$ read that Done $\left[\right.$ prev] is true (Line 29) or $p_{i}$ reads that Done $\left[\right.$ prev] is false and Lock $\left[p_{i}\right][$ prev.pid] is unlocked (Line 30 .

(Case 1) Suppose that $p_{i}$ read prev $=\perp$ and entered the critical section. Since in this case, $p_{i}$ does not have any predecessor, some other process that returns successfully from the while loop in Line 25 must 
Now consider a process $p_{i}$ that returns successfully from the while loop in Line 23. Suppose that $p_{i}$ is stuck indefinitely as it performs the while loop in Line 30. Thus, no process has unlocked the register $\operatorname{Lock}\left[p_{i}\right][$ prev.pid $]$ by writing to it in the Exit section. Recall that since $\left[p_{i}\right.$, face $\left._{i}\right]$ has reached the while loop in Line 30 $\left[p_{i}\right.$, face $\left._{i}\right]$ necessarily has a predecessor, say $\left[p_{j}, f a c e_{j}\right]$, and has set itself to be $p_{j}$ 's successor by writing $p_{i}$ to register $\operatorname{Succ}\left[p_{j}\right.$, face $\left._{j}\right]$ in Line 28, Consider the possible two cases: the predecessor of $\left[p_{j}\right.$, face $_{j}$ is some process $p_{k} ; k \neq i$ or the predecessor of $\left[p_{j}\right.$, face $_{j}$ is the process $p_{i}$ itself.

(Case 1) Since by assumption, process $p_{j}$ takes infinitely many steps in $E$, the only reason that $p_{j}$ is stuck without entering the critical section is that $\left[p_{k}\right.$, face $\left._{k}\right]$ is also stuck in the while loop in Line 30. Note that it is possible for us to iteratively extend this execution in which $p_{k}$ 's predecessor is a process that is not $p_{i}$ or $p_{j}$ that is also stuck in the while loop in Line 30. But then the last such process must eventually read the corresponding Lock to be unlocked and enter the critical section. Thus, in every extension of $E$ in which every process takes infinitely many steps, some process will enter the critical section.

(Case 2) Suppose that the predecessor of $\left[p_{j}\right.$, face $_{j}$ is the process $p_{i}$ itself. Thus, as $\left[p_{i}\right.$, face $_{]}$is stuck in the while loop waiting for $\operatorname{Lock}\left[p_{i}, p_{j}\right]$ to be unlocked by process $p_{j}, p_{j}$ leaves the critical section, unlocks $\operatorname{Lock}\left[p_{i}, p_{j}\right]$ in Line 37 and prior to the read of $\operatorname{Lock}\left[p_{i}, p_{j}\right], p_{j}$ re-starts the Entry operation, writes false to Done $\left[p_{j}, 1-\right.$ face $\left._{j}\right]$ and sets itself to be the successor of $\left[p_{i}\right.$, face $\left._{i}\right]$ and spins on the register $\operatorname{Lock}_{j}\left[p_{j}, p_{i}\right]$. However, observe that process $p_{i}$, which takes infinitely many steps by our assumption must eventually read that $\operatorname{Lock}\left[p_{i}, p_{j}\right]$ is unlocked and enter the critical section, thus establishing deadlock-freedom.

We say that a TM implementation $M$ accesses a single t-object if in every execution $E$ of $M$ and every transaction $T \in \operatorname{txns}(E),|\operatorname{Dset}(T)| \leq 1$. We can now prove the following theorem:

Theorem 4.19. Any strictly serializable, strongly progressive TM implementation $M$ that accesses a single t-object implies a deadlock-free, finite exit mutual exclusion implementation $L(M)$ such that the $R M R$ complexity of $M$ is within a constant factor of the RMR complexity of $L(M)$.

\section{Proof. (Mutual-exclusion) Follows from Lemma 4.17}

(Finite-exit) The proof is immediate since the Exit operation contains no unbounded loops or waiting statements.

(Deadlock-freedom) Follows from Lemma 4.18

(RMR complexity) First, let us consider the CC model. Observe that every event not on $M$ performed by a process $p_{i}$ as it performs the Entry or Exit operations incurs $O(1)$ RMR cost clearly, possibly barring the while loop executed in Line 30 . During the execution of this while loop, process $p_{i}$ spins on the register $\operatorname{Lock}\left[p_{i}\right]\left[p_{j}\right]$, where $p_{j}$ is the predecessor of $p_{i}$. Observe that $p_{i}$ 's cached copy of $\operatorname{Lock}\left[p_{i}\right]\left[p_{j}\right]$ may be invalidated only by process $p_{j}$ as it unlocks the register in Line 37. Since no other process may write to this register and $p_{i}$ terminates the while loop immediately after the write to $\operatorname{Lock}\left[p_{i}\right]\left[p_{j}\right]$ by $p_{j}, p_{i}$ incurs $O(1)$ RMR's. Thus, the overall RMR cost incurred by $M$ is within a constant factor of the RMR cost of $L(M)$.

Now we consider the DSM model. As with the reasoning for the CC model, every event not on $M$ performed by a process $p_{i}$ as it performs the Entry or Exit operations incurs $O(1)$ RMR cost clearly, possibly barring the while loop executed in Line 30 . During the execution of this while loop, process $p_{i}$ spins on the register $\operatorname{Lock}\left[p_{i}\right]\left[p_{j}\right]$, where $p_{j}$ is the predecessor of $p_{i}$. Recall that $\operatorname{Lock}\left[p_{i}\right]\left[p_{j}\right]$ is a register that is local to $p_{i}$ and thus, $p_{i}$ does not incur any RMR cost on account of executing this loop. It follows that $p_{i}$ incurs $O(1)$ RMR cost in the DSM model. Thus, the overall RMR cost of $M$ is within a constant factor of the RMR cost of $L(M)$ in the DSM model.

Theorem 4.20. ([21]) Any deadlock-free, finite-exit mutual exclusion implementation from read, write and conditional primitives has an execution whose $R M R$ complexity is $\Omega(n \log n)$.

Theorems 4.20 and 4.19 imply: 
Theorem 4.21. Any strictly serializable, strongly progressive TM implementation with wait-free TMliveness from read, write and conditional primitives that accesses a single t-object has an execution whose $R M R$ complexity is $\Omega(n \log n)$.

\subsubsection{A constant expensive synchronization opaque TM}

In this section, we describe a strongly progressive opaque TM implementation providing starvation-free TM-liveness from read-write base objects with constant RAW/AWAR cost. For our implementation, we define and implement a starvation-free multi-trylock object.

Starvation-free multi-trylock. A multi-trylock provides exclusive write-access to a set $Q$ of t-objects. Specifically, a multi-trylock exports the following operations

- acquire $(Q)$ returns true or false

- release $(Q)$ releases the lock and returns ok

- isContended $\left(X_{j}\right), X_{j} \in Q$ returns true or false

We assume that processes are well-formed: they never invoke a new operation on the multi-trylock before receiving response from the previous invocation.

We say that a process $p_{i}$ holds a lock on $X_{j}$ after an execution $\pi$ if $\pi$ contains the invocation of acquire $(Q)$, $X_{j} \in Q$ by $p_{i}$ that returned true, but does not contain a subsequent invocation of release $\left(Q^{\prime}\right), X_{j} \in Q^{\prime}$, by $p_{i}$ in $\pi$. We say that $X_{j}$ is locked after $\pi$ by process $p_{i}$ if $p_{i}$ holds a lock on $X_{j}$ after $\pi$.

We say that $X_{j}$ is contended by $p_{i}$ after an execution $\pi$ if $\pi$ contains the invocation of acquire $(Q), X_{j} \in Q$, by $p_{i}$ but does not contain a subsequent return false or return of release $\left(Q^{\prime}\right), X_{j} \in Q^{\prime}$, by $p_{i}$ in $\pi$.

Let an execution $\pi$ contain the invocation $i_{o p}$ of an operation op followed by a corresponding response $r_{o p}$ (we say that $\pi$ contains op). We say that $X_{j}$ is uncontended (resp., locked) during the execution of op in $\pi$ if $X_{j}$ is uncontended (resp., locked) after every prefix of $\pi$ that contains $i_{o p}$ but does not contain $r_{\text {op }}$.

We implement a multi-trylock object whose operations are starvation-free. The algorithm is inspired by the Black-White Bakery Algorithm [118, and uses a finite number of bounded registers.

A starvation-free multi-trylock implementation satisfies the following properties:

- Mutual-exclusion: For any object $X_{j}$, and any execution $\pi$, there exists at most one process that holds a lock on $X_{j}$ after $\pi$.

- Progress: Let $\pi$ be any execution that contains acquire $(Q)$ by process $p_{i}$. If no other process $p_{k}, k \neq i$ contends infinitely long on some $X_{j} \in Q$, then acquire $(Q)$ returns true in $\pi$.

- Let $\pi$ be any execution that contains is Contended $\left(X_{j}\right)$ invoked by $p_{i}$.

- If $X_{j}$ is locked by $p_{\ell} ; \ell \neq i$ during the complete execution of isContended $\left(X_{j}\right)$ in $\pi$, then is Contended $\left(X_{j}\right)$ returns true.

- If $\forall \ell \neq i, X_{j}$ is never contended by $p_{\ell}$ during the execution of isContended $\left(X_{j}\right)$ in $\pi$, then is Contended $\left(X_{j}\right)$ returns false.

Our starvation-free multi-trylock in Algorithm 4.4 uses the following shared variables: registers $r_{i j}$ for each process $p_{i}$ and object $X_{j}$, a shared bit color $\in\{B, W\}$, registers $L A_{i} \in\{0, \ldots, N\}$ for each $p_{i}$ that denote a Label and $M C_{i} \in\{B, W\}$ for each $p_{i}$.

We say $\left(L A_{i}, i\right)<\left(L A_{k}, k\right)$ iff $L A_{i}<L A_{k}$ or $L A_{i}=L A_{k}$ and $i<k$. We now prove the following invariant about the multi-trylock implementation.

Lemma 4.22. In every execution $\pi$ of Algorithm 4.4. if $p_{i}$ holds a lock on some object $X_{j}$ after $\pi$, then one of the following conditions must hold: 


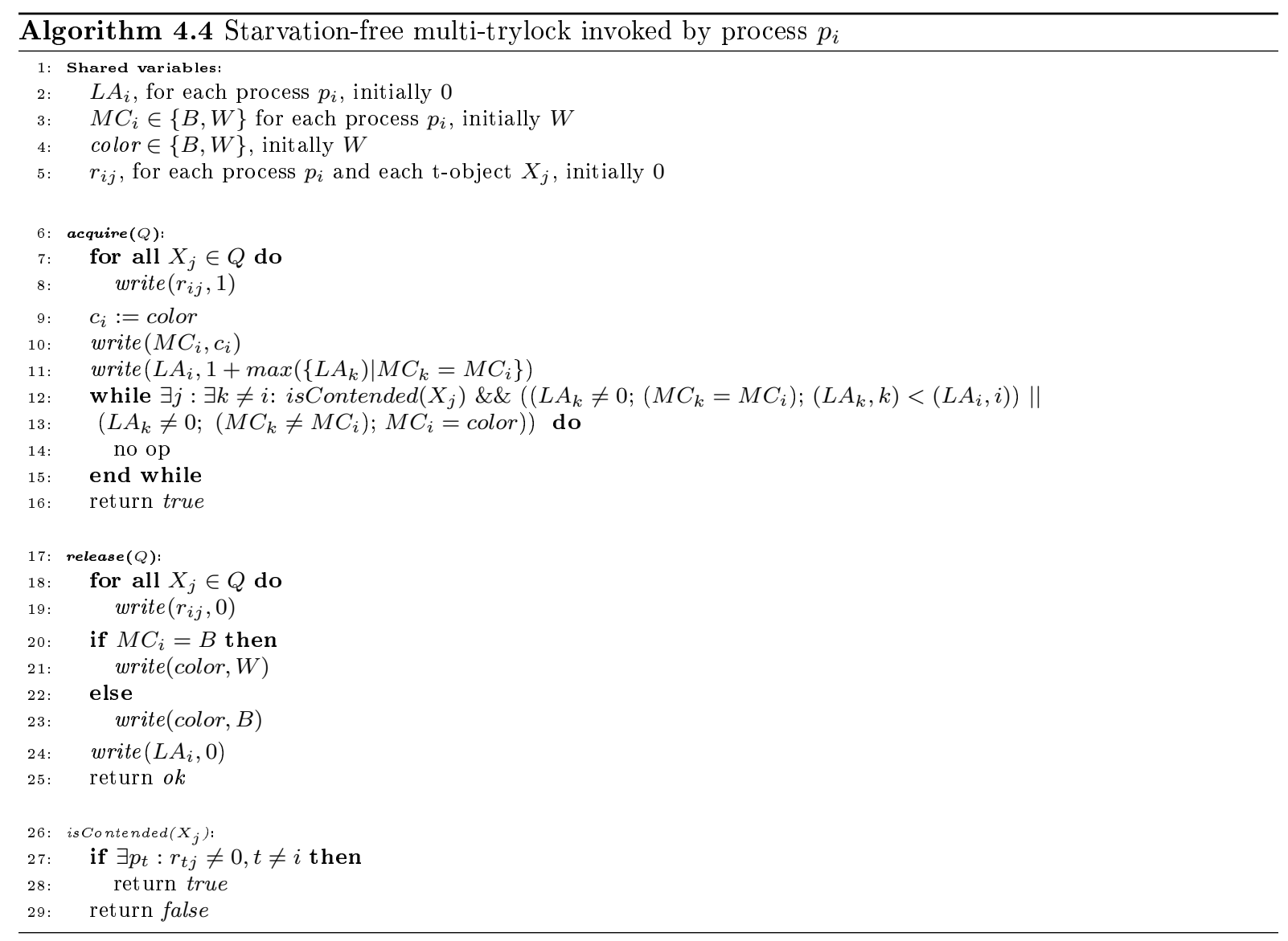

(1) for some $k \neq i ; L A_{k} \neq 0$, if $M C_{k}=M C_{i}$, then $\left(L A_{k}, k\right)>\left(L A_{i}, i\right)$

(2) for some $k \neq i$; $L A_{k} \neq 0$, if $M C_{k} \neq M C_{i}$, then $M C_{i} \neq$ color

Proof. In order to hold the lock on $X_{j}$, some process $p_{i}$ writes 1 to $r_{i j}$, writes a value, say $W$ to $M C_{i}$ and reads the Labels of other processes that have obtained the same color as itself and generates a Label greater by one than the maximum Label read (Line 11). Observe that until the value of the color bit is changed, all processes read the same value $W$. The first process $p_{i}$ to hold the lock on $X_{j}$ changes the color bit to $B$ when releasing the lock and hence the value read by all subsequent processes will be $B$ until it is changed again. Now consider two cases:

(1) Assume that there exists a process $p_{k}, k \neq i, L A_{k} \neq 0$ and $M C_{k}=M C_{i}$ such that $\left(L A_{k}, k\right)<$ $\left(L A_{i}, i\right)$, but $p_{i}$ holds a lock on $X_{j}$ after $\pi$. Thus, isContended $\left(X_{j}\right)$ returns true to $p_{i}$ because $p_{k}$ writes to $r_{k j}$ (Line 8 before writing to $L A_{k}$ (Line 11). By assumption, $\left(L A_{k}, k\right)<\left(L A_{i}, i\right) ; L A_{k}>$ 0 and $M C_{i}=M C_{k}$, but the conditional in Line 13 returned true to $p_{i}$ without waiting for $p_{k}$ to stop contending on $X_{j}$-contradiction.

(2) Assume that there exists a process $p_{k}, k \neq i, L A_{k} \neq 0$ and $M C_{k} \neq M C_{i}$ such that $M C_{i}=$ color, but $p_{i}$ holds a lock on $X_{j}$ after $\pi$. Again, since $L A_{k}>0$, isContended $\left(X_{j}\right)$ returns true to $p_{i}$, $M C_{k} \neq M C_{i}$ and $M C_{i}=$ color, but the conditional in Line 13 returned true to $p_{i}$ without waiting for $p_{k}$ to stop contending on $X_{j}$-contradiction.

We can thus prove the following theorem:

Theorem 4.23. Algorithm 4.4 is an implementation of multi-trylock object in which every operation is starvation-free and incurs at most four RAWs. 
Proof. Denote by $L$ the shared object implemented by Algorithm 4.4 .

Assume, by contradiction, that $L$ does not provide mutual-exclusion: there exists an execution $\pi$ after which processes $p_{i}$ and $p_{k}, k \neq i$ hold a lock on the same object, say $X_{j}$. Since both $p_{i}$ and $p_{k}$ have performed the write to $L A_{i}$ and $L A_{k}$ resp. in Line 11] $L A_{i}, L A_{k}>0$. Consider two cases:

(1) If $M C_{k}=M C_{i}$, then from Condition 1 of Lemma 4.22 we have $\left(L A_{k}, k\right)<\left(L A_{i}, i\right)$ and $\left(L A_{k}, k\right)>$ $\left(L A_{i}, i\right)$-contradiction.

(2) If $M C_{k} \neq M C_{i}$, then from Condition 2 of Lemma 4.22 we have $M C_{i} \neq$ color and $M C_{k} \neq$ color which implies $M C_{k}=M C_{i}$-contradiction.

$L$ also ensures progress. If process $p_{i}$ wants to hold the lock on an object $X_{j}$ i.e. invokes acquire $(Q), X_{j} \in$ $Q$, it checks if any other process $p_{k}$ holds the lock on $X_{j}$. If such a process $p_{k}$ exists and $M C_{k}=M C_{i}$, then clearly isContended $\left(X_{j}\right)$ returns true for $p_{i}$ and $\left(L A_{k}, k\right)<\left(L A_{i}, i\right)$. Thus, $p_{i}$ fails the conditional in Line 13 and waits until $p_{k}$ releases the lock on $X_{j}$ to return true. However, if $p_{k}$ contends infinitely long on $X_{j}, p_{i}$ is also forced to wait indefinitely to be returned true from the invocation of acquire $(Q)$. The same argument works when $M C_{k} \neq M C_{i}$ since when $p_{k}$ stops contending on $X_{j}$, isContended $\left(X_{j}\right)$ eventually returns false for $p_{i}$ if $p_{k}$ does not contend infinitely long on $X_{j}$.

All operations performed by $L$ are starvation-free. Each process $p_{i}$ that successfully holds the lock on an object $X_{j}$ in an execution $\pi$ invokes acquire $(Q), X_{j} \in Q$, obtains a color and chooses a value for $L A_{i}$ since there is no way to be blocked while writing to $L A_{i}$. The response of operation acquire $(Q)$ by $p_{i}$ is only delayed if there exists a concurrent invocation of acquire $\left(Q^{\prime}\right), X_{j} \in Q^{\prime}$ by $p_{k}$ in $\pi$. In that case, process $p_{i}$ waits until $p_{k}$ invokes release $(Q)$ and writes 0 to $r_{k j}$ and eventually holds the lock on $X_{j}$. The implementation of release and isContended are wait-free operations (and hence starvation-free) since they contains no unbounded loops or waiting statements.

The implementation of isContended $\left(X_{j}\right)$ only reads base objects. The implementation of release $(Q)$ writes to a series of base objects (Line 18) and then reads a base object (Line 20) incurring a single RAW. The implementation of acquire $(Q)$ writes to base objects (Line 8), reads the shared bit color (Line 9) - one RAW, writes to a base object (Line 10), reads the Labels (Line 11) — one RAW, writes to its own Label and finally performs a sequence of reads when evaluating the conditional in Line 13 - one RAW.

Thus, Algorithm 4.4 incurs at most four RAWs.

Strongly progressive TM from starvation-free multi-trylock. We now use the starvation-free multi-trylock to implement a starvation-free strongly progressive opaque TM implementation with constant expensive synchronization (Algorithm 4.5). The implementation is almost identical to the progressive TM implementation $L P$ in Algorithm 4.1 except that the function calls to acquire and release the transaction's write set are replaced with analogous calls to a multi-trylock object.

Theorem 4.24. Algorithm 4.5 implements a strongly progressive opaque TM implementation with starvation-free t-operations that uses invisible reads and employs at most four $R A W$ s per transaction.

Proof. (Opacity) Since Algorithm 4.5 is similar to the opaque progressive TM implementation in Algorithm 4.1 it is easy to adapt the proof of Lemma 4.9 to prove opacity for this implementation.

(TM-progress and TM-liveness) Every transaction $T_{k}$ in a TM $M$ whose t-operations are defined by Algorithm 4.5 can be aborted in the following scenarios:

- Read-validation failed in $\operatorname{read}_{k}$ or $\operatorname{try} C_{k}$

- $\operatorname{read}_{k}$ or $\operatorname{try} C_{k}$ returned $A_{k}$ because $X_{j} \in R \operatorname{set}\left(T_{k}\right)$ is locked (belongs to write set of a concurrent transaction) 


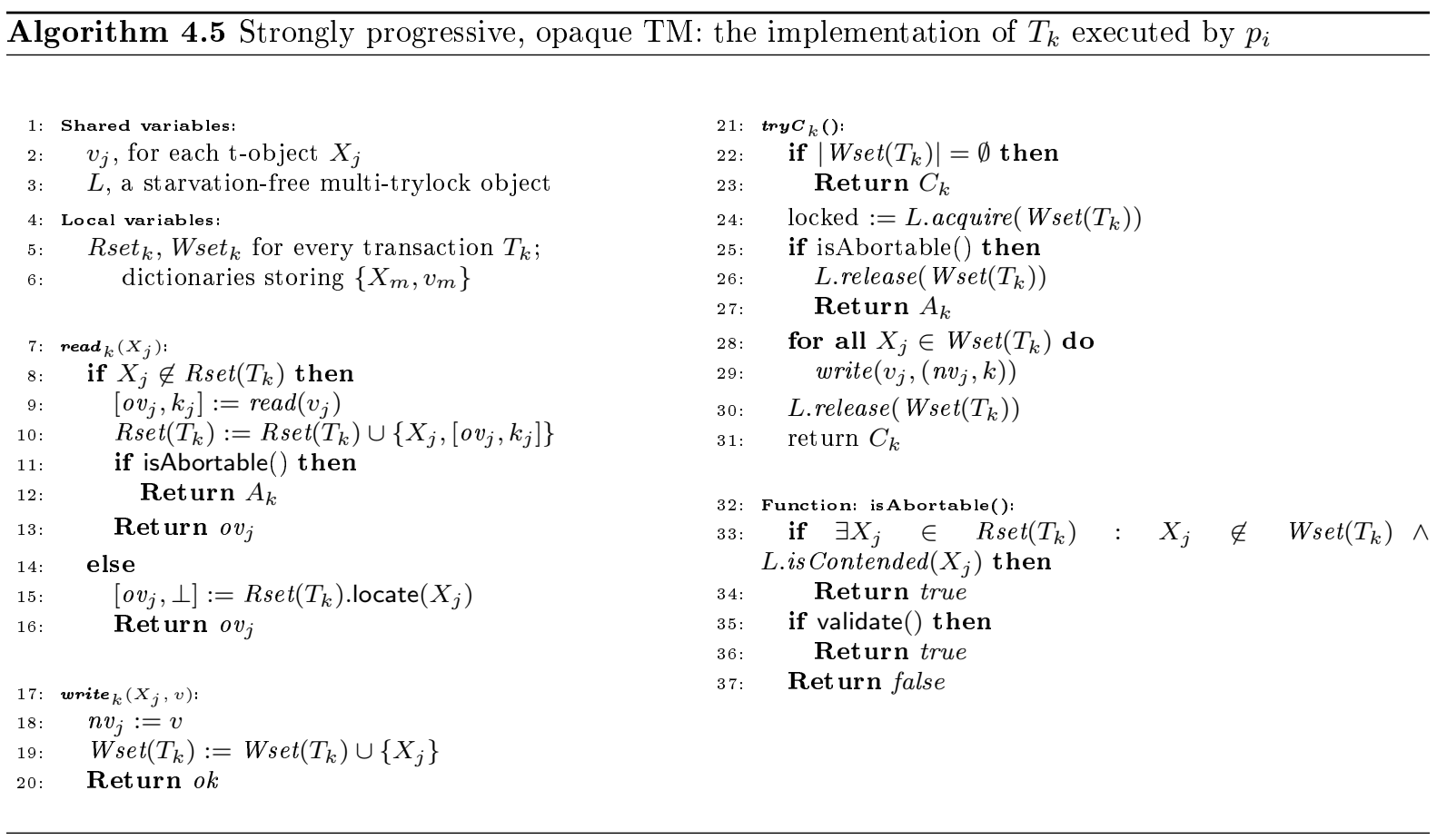

Since in each of these cases, a transaction is aborted only because of a read-write conflict with a concurrent transaction, it is easy to see that $M$ is progressive.

To show Algorithm 4.5 also implements a strongly progressive TM, we need to show that for every set of transactions that concurrently contend on a single t-object, at least one of the transactions is not aborted.

Consider transactions $T_{i}$ and $T_{k}$ that concurrently attempt to execute $\operatorname{try} C_{i}$ and $\operatorname{try} C_{k}$ such that $X_{j} \in$ Wset $_{i} \cup$ Wset $_{k}$. Consequently, they both invoke the acquire operation of the multi-trylock (Line 24) and thus, from Theorem 4.23 both $T_{i}$ and $T_{k}$ must commit eventually. Also, if validation of a t-read in $T_{k}$ fails, it means that the t-object is overwritten by some transaction $T_{i}$ such that $T_{i}$ precedes $T_{k}$, implying at least one of the transactions commit. Otherwise, if some t-object $X_{j} \in \operatorname{Rset}\left(T_{k}\right)$ is locked and returns abort since the t-object is in the write set of a concurrent transaction $T_{i}$. While it may still be possible that $T_{i}$ returns $A_{i}$ after acquiring the lock on $W_{s e} t_{i}$, strong progressiveness only guarantees progress for transactions that conflict on at most one t-object. Thus, in either case, for every set of transactions that conflict on at most one t-object, at least one transaction is not forcefully aborted.

Starvation-free TM-liveness follows from the fact that the multi try-lock we use in the implementation of $M$ provides starvation-free acquire and release operations.

(Complexity) Any process executing a transaction $T_{k}$ holds the lock on $W s e t\left(T_{k}\right)$ only once during $\operatorname{try} C_{k}$. If $\left|W \operatorname{set}\left(T_{k}\right)\right|=\emptyset$, then the transaction simply returns $C_{k}$ incurring no RAW's. Thus, from Theorem 4.23 Algorithm 4.5 incurs at most four RAWs per updating transaction and no RAW's are performed in read-only transactions.

\subsection{On the cost of permissive opaque TMs}

We have shown that (strongly) progressive TMs that allow a transaction to be aborted only on read-write conflicts have constant RAW/AWAR complexity. However, not aborting on conflicts may not necessarily affect TM-correctness. Ideally, we would like to derive TM implementations that are permissive, in the sense that a transaction is aborted only if committing it would violate TM-correctness. 
Definition 4.2 (Permissiveness). A TM implementation $M$ is permissive with respect to TM-correctness $C$ if for every history $H$ of $M$ such that $H$ ends with a response $r_{k}$ and replacing $r_{k}$ with some $r_{k} \neq A_{k}$ gives a history that satisfies $C$, we have $r_{k} \neq A_{k}$.

Therefore, permissiveness does not allow a transaction to abort, unless committing it would violate the execution's correctness.

We first show that a transaction in a permissive opaque implementation can only be forcefully aborted if it tries to commit:

Lemma 4.25. Let a TM implementation $M$ be permissive with respect to opacity. If a transaction $T_{i}$ is forcefully aborted executing a t-operation $\mathrm{op}_{i}$, then $\mathrm{op}_{i}$ is $\operatorname{try} C_{i}$.

Proof. Suppose, by contradiction, that there exists a history $H$ of $M$ such that some $o p_{i} \in\left\{\right.$ read $_{i}$, write $\left._{i}\right\}$ executed within a transaction $T_{i}$ returns $A_{i}$. Let $H_{0}$ be the shortest prefix of $H$ that ends just before $o p_{i}$ returns. By definition, $H_{0}$ is opaque and any history $H_{0} \cdot r_{i}$ where $r_{i} \neq A_{i}$ is not opaque. Let $H_{0}^{\prime}$ be the serialization of $H_{0}$.

If $o p_{i}$ is a write, then $H_{0} \cdot o k_{i}$ is also opaque - no write operation of the incomplete transaction $T_{i}$ appears in $H_{0}^{\prime}$ and, thus, $H_{0}^{\prime}$ is also a serialization of $H_{0} \cdot o k_{i}$.

If $o p_{i}$ is a $\operatorname{read}(X)$ for some t-object $X$, then we can construct a serialization of $H_{0} \cdot v$ where $v$ is the value of $X$ written by the last committed transaction in $H_{0}^{\prime}$ preceding $T_{i}$ or the initial value of $X$ if there is no such transaction. It is easy to see that $H_{0}$ " obtained from $H_{0}^{\prime}$ by adding $\operatorname{read}(X) \cdot v$ at the end of $T_{i}$ is a serialization of $H_{0} \cdot \operatorname{read}(X)$. In both cases, there exists a non- $A_{i}$ response $r_{i}$ to $o p_{i}$ that preserves opacity of $H_{0} \cdot r_{i}$, and, thus, the only t-operation that can be forcefully aborted in an execution of $M$ is $\operatorname{try} C$.

We now show that an execution of a transaction in a permissive opaque TM implementation (providing starvation-free TM-liveness) may require to perform at least one RAW/AWAR pattern per t-read.

Theorem 4.26. Let $M$ be a permissive opaque TM implementation providing starvation-free TMliveness. Then, for any $m \in \mathbb{N}, M$ has an execution in which some transaction performs $m$-reads such that the execution of each t-read contains at least one RAW or AWAR.

Proof. Consider an execution $E$ of $M$ consisting of transactions $T_{1}, T_{2}, T_{3}$ as shown in Figure 4.2 . $T_{3}$ performs a t-read of $X_{1}$, then $T_{2}$ performs a t-write on $X_{1}$ and commits, and finally $T_{1}$ performs a series of reads from objects $X_{1}, \ldots, X_{m}$. Since the implementation is permissive, no transaction can be forcefully aborted in $E$, and the only valid serialization of this execution is $T_{3}, T_{2}, T_{1}$. Note also that the execution generates a sequential history: each invocation of a t-operation is immediately followed by a matching response. Thus, since we assume starvation-freedom as a liveness property, such an execution exists.

We consider read $_{1}\left(X_{k}\right), 2 \leq k \leq m$ in execution $E$. Imagine that we modify the execution $E$ as follows. Immediately after read $d_{1}\left(X_{k}\right)$ executed by $T_{1}$ we add write $_{3}(X, v)$, and $t r y C_{3}$ executed by $T_{3}\left(\right.$ let $T C_{3}\left(X_{k}\right)$ denote the complete execution of $W_{3}\left(X_{k}, v\right)$ followed by $\left.\operatorname{try} C_{3}\right)$. Obviously, $T C_{3}\left(X_{k}\right)$ must return abort: neither $T_{3}$ can be serialized before $T_{1}$ nor $T_{1}$ can be serialized before $T_{3}$. On the other hand if $T C_{3}\left(X_{k}\right)$ takes place just before $\operatorname{read}_{1}\left(X_{k}\right)$, then $T C_{3}\left(X_{k}\right)$ must return commit but read $d_{1}\left(X_{k}\right)$ must return the value written by $T_{3}$. In other words, read $d_{1}\left(X_{k}\right)$ and $T C_{3}\left(X_{k}\right)$ are strongly non-commutative [16]: both of them see the difference when ordered differently. As a result, intuitively, $\operatorname{read}_{1}\left(X_{k}\right)$ needs to perform a RAW or AWAR to make sure that the order of these two "conflicting" operations is properly maintained. We formalize this argument below.

Consider a modification $E^{\prime}$ of $E$, in which $T_{3}$ performs write $e_{3}\left(X_{k}\right)$ immediately after $\operatorname{read}_{1}\left(X_{k}\right)$ and then tries to commit. In any serialization of $E^{\prime}, T_{3}$ must precede $T_{2}\left(\operatorname{read}_{3}\left(X_{1}\right)\right.$ returns the initial value of $\left.X_{1}\right)$ and $T_{2}$ must precede $T_{1}$ to respect the real-time order of transactions. The execution of $\operatorname{read}_{1}\left(X_{k}\right)$ does not modify base objects, hence, $T_{3}$ does not observe $\operatorname{read}_{1}\left(X_{k}\right)$ in $E^{\prime}$. Since $M$ is permissive, $T_{3}$ must commit in $E^{\prime}$. But since $T_{1}$ performs read $_{1}\left(X_{k}\right)$ before $T_{3}$ commits and $T_{3}$ updates $X_{k}$, we also have 


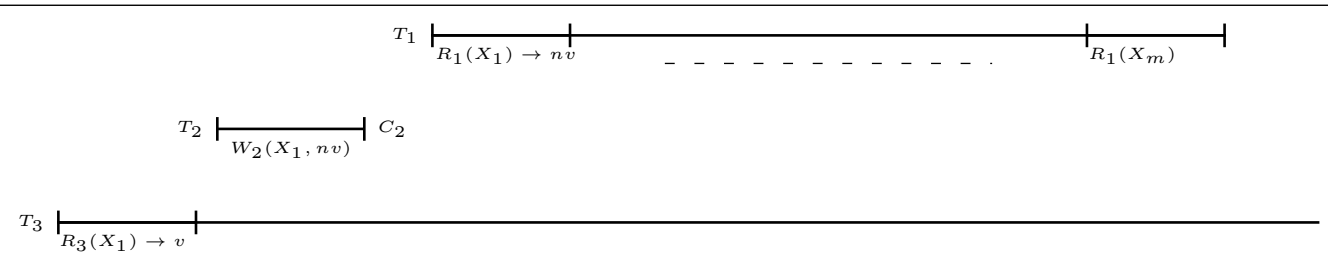

Figure 4.2: Execution $E$ of a permissive, opaque TM: $T_{2}$ and $T_{3}$ force $T_{1}$ to perform a RAW/AWAR in each $R_{1}\left(X_{k}\right), 2 \leq k \leq m$

$T_{1}$ must precede $T_{3}$ in any serialization. Thus, $T_{3}$ cannot precede $T_{1}$ in any serialization-contradiction. Consequently, each read $_{1}\left(X_{k}\right)$ must perform a write to a base object.

Let $\pi$ be the execution fragment that represents the complete execution of $\operatorname{read}_{1}\left(X_{k}\right)$ and $E^{k}$, the prefix of $E$ up to (but excluding) the invocation of $\operatorname{read}_{1}\left(X_{k}\right)$.

Clearly, $\pi$ contains a write to a base object. Let $\pi_{w}$ be the first write to a base object in $\pi$. Thus, $\pi$ can be represented as $\pi_{s} \cdot \pi_{w} \cdot \pi_{f}$. Suppose that $\pi$ does not contain a RAW or AWAR. Consider the execution fragment $E^{k} \cdot \pi_{s} \cdot \rho$, where $\rho$ is the complete execution of $T C_{3}\left(X_{k}\right)$ by $T_{3}$. Such an execution of $M$ exists since $\pi_{s}$ does not perform any base object write, hence, $E^{k} \cdot \pi_{s} \cdot \rho$ is indistinguishable to $T_{3}$ from $E^{k} \cdot \rho$.

Since, by our assumption, $\pi_{w} \cdot \pi_{f}$ contains no RAW, any read performed in $\pi_{w} \cdot \pi_{f}$ can only be applied to base objects previously written in $\pi_{w} \cdot \pi_{f}$. Since $\pi_{w}$ is not an AWAR, $E^{k} \cdot \pi_{s} \cdot \rho \cdot \pi_{w} \cdot \pi_{f}$ is an execution of $M$ since it is indistinguishable to $T_{1}$ from $E^{k} \cdot \pi$. In $E^{k} \cdot \pi_{s} \cdot \rho \cdot \pi_{w} \cdot \pi_{f}, T_{3}$ commits (as in $\rho$ ) but $T_{1}$ ignores the value written by $T_{3}$ to $X_{k}$. But there exists no serialization that justifies this execution - contradiction to the assumption that $M$ is opaque. Thus, each $\operatorname{read}_{1}\left(X_{k}\right), 2 \leq k \leq m$ must contain a RAW/AWAR.

Note that since all t-reads of $T_{1}$ are executed sequentially, all these RAW/AWAR patterns are pairwise non-overlapping, which completes the proof.

\subsection{Related work and Discussion}

In this section, we summarize the complexity bounds for blocking TMs presented in this chapter and identify some open questions.

Sequential TMs. Theorem 4.2 improves the read-validation step-complexity lower bound 60, 62. derived for strict-data partitioning (a very strong version of DAP) and invisible reads. In a strict data partitioned TM, the set of base objects used by the TM is split into disjoint sets, each storing information only about a single data item. Indeed, every TM implementation that is strict data-partitioned satisfies weak DAP, but not vice-versa (cf. Section 2.6). The definition of invisible reads assumed in 60, 62, requires that a t-read operation does not apply nontrivial events in any execution. Theorem 4.2 however, assumes weak invisible reads, stipulating that t-read operations of a transaction $T$ do not apply nontrivial events only when $T$ is not concurrent with any other transaction. We believe that the TM-progress and TM-liveness restrictions as well as the definitions of DAP and invisible reads we consider for this result are the weakest possible assumptions that may be made. To the best of our knowledge, these assumptions cover every TM implementation that is subject to the validation step-complexity [35, 38, 77.

Progressive TMs. We summarize the known complexity bounds for progressive (and resp. strongly progressive) TMs in Table 4.1 (and resp. Table 4.2). Some questions remain open. Can the tight bounds on step complexity for progressive TMs in Corollaries 4.14 and 4.16 be extended to strongly progressive TMs?

Guerraoui and Kapalka [62] proved that it is impossible to implement strictly serializable strongly progressive TMs that provide wait-free TM-liveness (every t-operation returns a matching response within 


\begin{tabular}{l|l|l|l|l|l|} 
TM-correctness & TM-liveness & DAP & Invisible reads & Read-write & Complexity \\
\hline Opacity & ICF & weak & yes & yes & $\Theta\left(\mid\right.$ Rset $\left.\left.\right|^{2}\right)$ step-complexity \\
\hline Strict serializability & ICF & weak & yes & yes & $\Theta(\mid$ Rset $\mid)$ step-complexity for tryCommit \\
\hline Opacity & WF & strict & yes & yes & $O(1)$ RAW/AWAR, O(1) stalls for t-reads \\
\hline Opacity & starvation-free & strict & & & $\Theta(\mid$ Wset $\mid)$ protected data
\end{tabular}

Table 4.1: Complexity bounds for progressive TMs.

\begin{tabular}{l|l|l|l|l|} 
TM-correctness & TM-liveness & Invisible reads & rmw primitives & Complexity \\
\hline Strict serializability & WF & & read-write & Impossible \\
\hline Strict serializability & & & read-write, conditional & $\Omega(n \log n)$ RMRs \\
\hline Opacity & starvation-free & yes & read-write & $O(1)$ RAW/AWAR
\end{tabular}

Table 4.2: Complexity bounds for strongly progressive TMs.

a finite number of steps) using only read and write primitives. Algorithm 4.5 describes one means to circumvent this impossibility result by describing an opaque strongly progressive TM implementation from read-write base objects that provides starvation-free TM-liveness.

We conjecture that the lower bound of Theorem 4.21 on the RMR complexity is tight. Proving this remains an interesting open question.

Permissive TMs. Crain et al. 33] proved that a permissive opaque TM implementation cannot maintain invisible reads, which inspired the derivation of our lower bound on RAW/AWAR complexity in Section 4.5. Furthermore, [33] described a permissive VWC TM implementation that ensures that t-read operations do not perform nontrivial primitives, but the tryCommit invoked by a read-only transaction perform a linear (in the size of the transaction's data set) number of RAW/AWARs. Thus, an open question is whether there exists a linear lower bound on RAW/AWAR complexity for weaker (than opacity) TM-correctness properties of VWC and TMS1. 



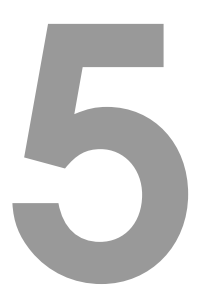

\section{Complexity bounds for non-blocking TMs}

\subsection{Overview}

In the previous chapter, we presented complexity bounds for lock-based blocking TMs. Early TM implementations such as the popular DSTM [77] however avoid using locks and provide non-blocking TM-progress. In this chapter, we present several complexity bounds for non-blocking TMs exemplified by obstruction-freedom, possibly the weakest non-blocking progress condition [76, 80].

We first establish that it is impossible to implement a strictly serializable obstruction-free TM that provides both weak DAP and read invisibility. Indeed, popular obstruction-free TMs like DSTM [77] and FSTM [51] are weak DAP, but use visible reads for aborting pending writing transactions. Secondly, we show that a t-read operation in a $n$-process strictly serializable obstruction-free TM implementation may incur $\Omega(n)$ stalls. Specifically, we prove that every such TM implementation has a $(n-1)$-stall execution for an invoked t-read operation. Thirdly, we prove that any RW DAP opaque obstructionfree TM implementation has an execution in which a read-only transaction incurs $\Omega(n)$ non-overlapping $R A W s$ or AWARs. Finally, we show that there exists a considerable complexity gap between blocking (i.e., progressive) and non-blocking (i.e., obstruction-free) TM implementations. We use the progressive opaque TM implementation LP described in Algorithm 4.1 (Chapter 4) to establish a linear separation in memory stall and RAW/AWAR complexity between blocking and non-blocking TMs.

Formally, let $\mathcal{O F}$ denote the class of TMs that provide OF TM-progress and OF TM-liveness.

Roadmap of Chapter 5. In Section 5.2, we show that no strictly serializable TM in $\mathcal{O F}$ can be weak DAP and have invisible reads. In Section 5.3 we determine stall complexity bounds for strictly serializable TMs in $\mathcal{O F}$, and in Section 5.4, we present a linear (in $n$ ) lower bound on the RAW/AWAR complexity for RW DAP opaque TMs in $\mathcal{O F}$. In Section 5.5 we describe two obstruction-free algorithms: a RW DAP opaque TM and a weak DAP (but not RW DAP) opaque TM. In Section 5.6 we present complexity gaps between blocking and non-blocking TM implementations. We conclude this chapter with a discussion on related work and open questions concerning obstruction-free TMs.

\subsection{Impossibility of weak DAP and invisible reads}

In this section, we prove that it is impossible to combine weak DAP and invisible reads for strictly serializable TMs in $\mathcal{O F}$. 


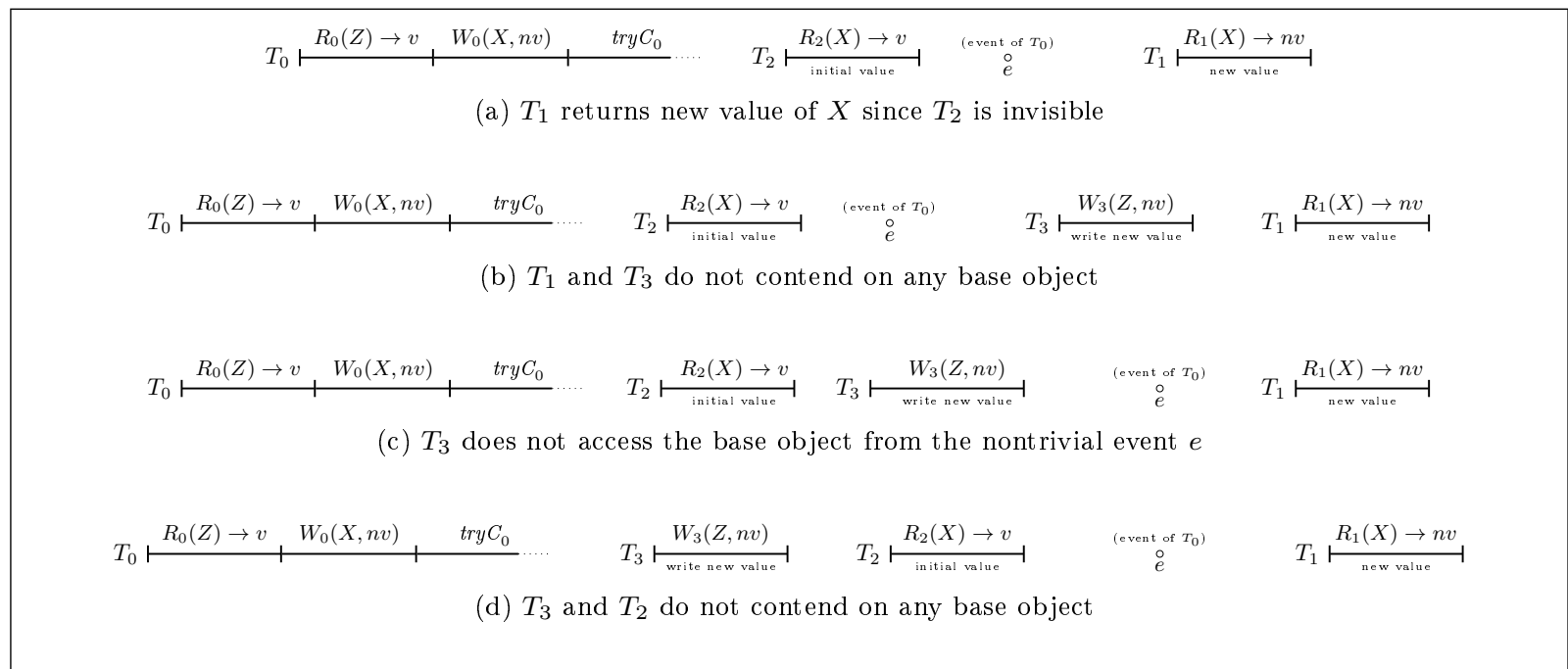

Figure 5.1: Executions in the proof of Theorem 5.1 execution in 5.1d is not strictly serializable

Here is a proof sketch: suppose, by contradiction, that such a TM implementation $M$ exists. Consider an execution $E$ of $M$ in which a transaction $T_{0}$ performs a t-read of t-object $Z$ (returning the initial value $v$ ), writes $n v$ (new value) to t-object $X$, and commits. Let $E^{\prime}$ denote the longest prefix of $E$ that cannot be extended with the t-complete step contention-free execution of any transaction that reads $n v$ in $X$ and commits.

Thus if $T_{0}$ takes one more step, then the resulting execution $E^{\prime} \cdot e$ can be extended with the t-complete step contention-free execution of a transaction $T_{1}$ that reads $n v$ in $X$ and commits.

Since $M$ uses invisible reads, the following execution exists: $E^{\prime}$ can be extended with the t-complete step contention-free execution of a transaction $T_{2}$ that reads the initial value $v$ in $X$ and commits, followed by the step $e$ of $T_{0}$ after which transaction $T_{1}$ running step contention-free reads $n v$ in $X$ and commits. Moreover, this execution is indistinguishable to $T_{1}$ and $T_{2}$ from an execution in which the read set of $T_{0}$ is empty. Thus, we can modify this execution by inserting the step contention-free execution of a committed transaction $T_{3}$ that writes a new value to $Z$ after $E^{\prime}$, but preceding $T_{2}$ in real-time order. Intuitively, by weak DAP, transactions $T_{1}$ and $T_{2}$ cannot distinguish this execution from the original one in which $T_{3}$ does not participate.

Thus, we can show that the following execution exists: $E^{\prime}$ is extended with the t-complete step contentionfree execution of $T_{3}$ that writes $n v$ to $Z$ and commits, followed by the t-complete step contention-free execution of $T_{2}$ that reads the initial value $v$ in $X$ and commits, followed by the step $e$ of $T_{0}$, after which $T_{1}$ reads $n v$ in $X$ and commits.

This execution is, however, not strictly serializable: $T_{0}$ must appear in any serialization $\left(T_{1}\right.$ reads a value written by $T_{0}$ ). Transaction $T_{2}$ must precede $T_{0}$, since the t-read of $X$ by $T_{2}$ returns the initial value of $X$. To respect real-time order, $T_{3}$ must precede $T_{2}$. Finally, $T_{0}$ must precede $T_{3}$ since the t-read of $Z$ returns the initial value of $Z$. The cycle $T_{0} \rightarrow T_{3} \rightarrow T_{2} \rightarrow T_{0}$ implies a contradiction.

The formal proof follows.

Theorem 5.1. There does not exist a weak DAP strictly serializable TM implementation in $\mathcal{O F}$ that uses invisible reads.

Proof. By contradiction, assume that such an implementation $M \in \mathcal{O F}$ exists. Let $v$ be the initial value of t-objects $X$ and $Z$. Consider an execution $E$ of $M$ in which a transaction $T_{0}$ performs read $_{0}(Z) \rightarrow v$ (returning $v$ ), writes $n v \neq v$ to $X$, and commits. Let $E^{\prime}$ denote the longest prefix of $E$ that cannot be extended with the t-complete step contention-free execution of any transaction performing a t-read $X$ that returns $n v$ and commits. 
Let $e$ be the enabled event of transaction $T_{0}$ in the configuration after $E^{\prime}$. Without loss of generality, assume that $E^{\prime} \cdot e$ can be extended with the t-complete step contention-free execution of a committed transaction $T_{1}$ that reads $X$ and returns $n v$. Let $E^{\prime} \cdot e \cdot E_{1}$ be such an execution, where $E_{1}$ is the t-complete step contention-free execution fragment of transaction $T_{1}$ that performs $\operatorname{read}_{1}(X) \rightarrow n v$ and commits.

We now prove that $M$ has an execution of the form $E^{\prime} \cdot E_{2} \cdot e \cdot E_{1}$, where $E_{2}$ is the t-complete step contention-free execution fragment of transaction $T_{2}$ that performs $\operatorname{read}_{2}(X) \rightarrow v$ and commits.

We observe that $E^{\prime} \cdot E_{2}$ is an execution of $M$. Indeed, by OF TM-progress and OF TM-liveness, $T_{2}$ must return a matching response that is not $A_{2}$ in $E^{\prime} \cdot E_{2}$, and by the definition of $E^{\prime}$, this response must be the initial value $v$ of $X$.

By the assumption of invisible reads, $E_{2}$ does not contain any nontrivial events. Consequently, $E^{\prime} \cdot E_{2} \cdot e \cdot E_{1}$ is indistinguishable to transaction $T_{1}$ from the execution $E^{\prime} \cdot e \cdot E_{1}$. Thus, $E^{\prime} \cdot E_{2} \cdot e \cdot E_{1}$ is also an execution of $M$ (Figure 5.1a).

Claim 5.2. $M$ has an execution of the form $E^{\prime} \cdot E_{2} \cdot E_{3} \cdot e \cdot E_{1}$ where $E_{3}$ is the $t$-complete step contentionfree execution fragment of transaction $T_{3}$ that writes $n v \neq v$ to $Z$ and commits.

Proof. The proof is through a sequence of indistinguishability arguments to construct the execution.

We first claim that $M$ has an execution of the form $E^{\prime} \cdot E_{2} \cdot e \cdot E_{1} \cdot E_{3}$. Indeed, by OF TM-progress and OF TM-liveness, $T_{3}$ must be committed in $E^{\prime} \cdot E_{2} \cdot e \cdot E_{1} \cdot E_{3}$.

Since $M$ uses invisible reads, the execution $E^{\prime} \cdot E_{2} \cdot e \cdot E_{1} \cdot E_{3}$ is indistinguishable to transactions $T_{1}$ and $T_{3}$ from the execution $\hat{E} \cdot E_{1} \cdot E_{3}$, where $\hat{E}$ is the t-incomplete step contention-free execution of transaction $T_{0}$ with $\operatorname{Wset}_{\hat{E}}\left(T_{0}\right)=\{X\} ; \operatorname{Rset}_{\hat{E}}\left(T_{0}\right)=\emptyset$ that writes $n v$ to $X$.

Observe that the execution $E^{\prime} \cdot E_{2} \cdot e \cdot E_{1} \cdot E_{3}$ is indistinguishable to transactions $T_{1}$ and $T_{3}$ from the execution $\hat{E} \cdot E_{1} \cdot E_{3}$, in which transactions $T_{3}$ and $T_{1}$ are disjoint-access. Consequently, by Lemma 2.10 $T_{1}$ and $T_{3}$ do not contend on any base object in $\hat{E} \cdot E_{1} \cdot E_{3}$. Thus, $M$ has an execution of the form $E^{\prime} \cdot E_{2} \cdot e \cdot E_{3} \cdot E_{1}$ (Figure 5.1b.

By definition of $E^{\prime}, T_{0}$ applies a nontrivial primitive to some base object, say $b$, in event $e$ that $T_{1}$ must access in $E_{1}$. Thus, the execution fragment $E_{3}$ does not contain any nontrivial event on $b$ in the execution $E^{\prime} \cdot E_{2} \cdot e \cdot E_{1} \cdot E_{3}$. In fact, since $T_{3}$ is disjoint-access with $T_{0}$ in the execution $\hat{E} \cdot E_{3} \cdot E_{1}$, by Lemma 2.10, it cannot access the base object $b$ to which $T_{0}$ applies a nontrivial primitive in the event $e$. Thus, transaction $T_{3}$ must perform the same sequence of events $E_{3}$ immediately after $E^{\prime}$, implying that $M$ has an execution of the form $E^{\prime} \cdot E_{2} \cdot E_{3} \cdot e \cdot E_{1}$ (Figure 5.1c).

Finally, we observe that the execution $E^{\prime} \cdot E_{2} \cdot E_{3} \cdot e \cdot E_{1}$ established in Claim 5.2 is indistinguishable to transactions $T_{2}$ and $T_{3}$ from an execution $\tilde{E} \cdot E_{2} \cdot E_{3} \cdot e \cdot E_{1}$, where $W \operatorname{set}\left(T_{0}\right)=\{X\}$ and $R \operatorname{set}\left(T_{0}\right)=\emptyset$ in $\tilde{E}$. But transactions $T_{3}$ and $T_{2}$ are disjoint-access in $\tilde{E} \cdot E_{2} \cdot E_{3} \cdot e \cdot E_{1}$ and by Lemma $2.10, T_{2}$ and $T_{3}$ do not contend on any base object in this execution. Thus, $M$ has an execution of the form $E^{\prime} \cdot E_{3} \cdot E_{2} \cdot e \cdot E_{1}$ (Figure 5.1d) in which $T_{3}$ precedes $T_{2}$ in real-time order.

However, the execution $E^{\prime} \cdot E_{3} \cdot E_{2} \cdot e \cdot E_{1}$ is not strictly serializable: $T_{0}$ must be committed in any serialization and transaction $T_{2}$ must precede $T_{0}$ since read $_{2}(X)$ returns the initial value of $X$. To respect real-time order, $T_{3}$ must precede $T_{2}$, while $T_{0}$ must precede $T_{1}$ since read $d_{1}(X)$ returns $n v$, the value of $X$ updated by $T_{0}$. Finally, $T_{0}$ must precede $T_{3}$ since $\operatorname{read}_{0}(Z)$ returns the initial value of $Z$. But there exists no such serialization-contradiction. 


\subsection{A linear lower bound on memory stall complexity}

We prove a linear (in $n$ ) lower bound for strictly serializable TM implementations in $\mathcal{O} \mathcal{F}$ on the total number of memory stalls incurred by a single t-read operation.

Inductively, for each $k \leq n-1$, we construct a specific $k$-stall execution [45] in which some t-read operation by a process $p$ incurs $k$ stalls. In the $k$-stall execution, $k$ processes are partitioned into disjoint subsets $S_{1}, \ldots, S_{i}$. The execution can be represented as $\alpha \cdot \sigma_{1} \cdots \sigma_{i} ; \alpha$ is $p$-free, where in each $\sigma_{j}$, $j=1, \ldots, i, p$ first runs by itself, then each process in $S_{j}$ applies a nontrivial event on a base object $b_{j}$, and then $p$ applies an event on $b_{j}$. Moreover, $p$ does not detect step contention in this execution and, thus, must return a non-abort value in its t-read and commit in the solo extension of it. Additionally, it is guaranteed that in any extension of $\alpha$ by the processes other than $\{p\} \cup S_{1} \cup S_{2} \cup \ldots \cup S_{i}$, no nontrivial primitive is applied on a base object accessed in $\sigma_{1} \cdots \sigma_{i}$.

Assuming that $k \leq n-2$, we introduce a not previously used process executing an updating transaction immediately after $\alpha$, so that the subsequent t-read operation executed by $p$ is "perturbed" (must return another value). This will help us to construct a $\left(k+k^{\prime}\right)$-stall execution $\alpha \cdot \alpha^{\prime} \cdot \sigma_{1} \cdots \sigma_{i} \cdot \sigma_{i+1}$, where $k^{\prime}>0$.

The formal proof follows:

Theorem 5.3. Every strictly serializable TM implementation $M \in \mathcal{O} \mathcal{F}$ has a $(n-1)$-stall execution $E$ for a t-read operation performed in $E$.

Proof. We proceed by induction. Observe that the empty execution is a 0-stall execution since it vacuously satisfies the invariants of Definition 2.18.

Let $v$ be the initial value of t-objects $X$ and $Z$. Let $\alpha=\alpha_{1} \cdots \alpha_{n-2}$ be a step contention-free execution of a strictly serializable TM implementation $M \in \mathcal{O F}$, where for all $j \in\{1, \ldots, n-2\}$, $\alpha_{j}$ is the longest prefix of the execution fragment $\bar{\alpha}_{j}$ that denotes the t-complete step-contention free execution of committed transaction $T_{j}$ (invoked by process $p_{j}$ ) that performs $\operatorname{read}_{j}(Z) \rightarrow v$, writes value $n v \neq v$ to $X$ in the execution $\alpha_{1} \cdots \alpha_{j-1} \cdot \bar{\alpha}_{j}$ such that

- $\operatorname{try} C_{j}()$ is incomplete in $\alpha_{j}$,

- $\alpha_{1} \cdots \alpha_{j}$ cannot be extended with the t-complete step contention-free execution fragment of any transaction $T_{n-1}$ or $T_{n}$ that performs exactly one t-read of $X$ that returns $n v$ and commits.

Assume, inductively, that $\alpha \cdot \sigma_{1} \cdots \sigma_{i}$ is a $k$-stall execution for $\operatorname{read}_{n}(X)$ executed by process $p_{n}$, where $0 \leq k \leq n-2$. By Definition 2.18 there are distinct base objects $b_{1}, \ldots b_{i}$ accessed by disjoint sets of processes $S_{1} \ldots S_{i}$ in the execution fragment $\sigma_{1} \cdots \sigma_{i}$, where $\left|S_{1} \cup \ldots \cup S_{i}\right|=k$ and $\sigma_{1} \cdots \sigma_{i}$ contains no events of processes not in $S_{1} \cup \ldots \cup S_{i} \cup\left\{p_{n}\right\}$. We will prove that there exists a $\left(k+k^{\prime}\right)$-stall execution for $\operatorname{read}_{n}(X)$, for some $k^{\prime} \geq 1$.

By Lemma 2.12 $\alpha \cdot \sigma_{1} \cdots \sigma_{i}$ is indistinguishable to $T_{n}$ from a step contention-free execution. Let $\sigma$ be the finite step contention-free execution fragment that extends $\alpha \cdot \sigma_{1} \cdots \sigma_{i}$ in which $T_{n}$ performs events by itself: completes $\operatorname{read}_{n}(X)$ and returns a response. By OF TM-progress and OF TM-liveness, $\operatorname{read}_{n}(X)$ and the subsequent $\operatorname{try} C_{k}$ must each return non- $A_{n}$ responses in $\alpha \cdot \sigma_{1} \cdots \sigma_{i} \cdot \sigma$. By construction of $\alpha$ and strict serializability of $M, \operatorname{read}_{n}(X)$ must return the response $v$ or $n v$ in this execution. We prove that there exists an execution fragment $\gamma$ performed by some process $p_{n-1} \notin\left(\left\{p_{n}\right\} \cup S_{1} \cup \cdots \cup S_{i}\right)$ extending $\alpha$ that contains a nontrivial event on some base object that must be accessed by $\operatorname{read}_{n}(X)$ in $\sigma_{1} \cdots \sigma_{i} \cdot \sigma$.

Consider the case that $\operatorname{read}_{n}(X)$ returns the response $n v$ in $\alpha \cdot \sigma_{1} \cdots \sigma_{i} \cdot \sigma$. We define a step contentionfree fragment $\gamma$ extending $\alpha$ that is the t-complete step contention-free execution of transaction $T_{n-1}$ executed by some process $p_{n-1} \notin\left(\left\{p_{n}\right\} \cup S_{1} \cup \cdots \cup S_{i}\right)$ that performs $\operatorname{read}_{n-1}(X) \rightarrow v$, writes $n v \neq v$ to $Z$ and commits. By definition of $\alpha$, OF TM-progress and OF TM-liveness, $M$ has an execution of the form $\alpha \cdot \gamma$. We claim that the execution fragment $\gamma$ must contain a nontrivial event on some base object that must be accessed by $\operatorname{read}_{n}(X)$ in $\sigma_{1} \cdots \sigma_{i} \cdot \sigma$. Suppose otherwise. Then, $\operatorname{read}_{n}(X)$ must return the response $n v$ in $\sigma_{1} \cdots \sigma_{i} \cdot \sigma$. But the execution $\alpha \cdot \sigma_{1} \cdots \sigma_{i} \cdot \sigma$ is not strictly serializable. Since 
$\operatorname{read}_{n}(X) \rightarrow n v$, there exists a transaction $T_{q} \in \operatorname{txns}(\alpha)$ that must be committed and must precede $T_{n}$ in any serialization. Transaction $T_{n-1}$ must precede $T_{n}$ in any serialization to respect the real-time order and $T_{n-1}$ must precede $T_{q}$ in any serialization. Also, $T_{q}$ must precede $T_{n-1}$ in any serialization. But there exists no such serialization.

Consider the case that $\operatorname{read}_{n}(X)$ returns the response $v$ in $\alpha \cdot \sigma_{1} \cdots \sigma_{i} \cdot \sigma$. In this case, we define the step contention-free fragment $\gamma$ extending $\alpha$ as the t-complete step contention-free execution of transaction $T_{n-1}$ executed by some process $p_{n-1} \notin\left(\left\{p_{n}\right\} \cup S_{1} \cup \cdots \cup S_{i}\right)$ that writes $n v \neq v$ to $X$ and commits. By definition of $\alpha$, OF TM-progress and OF TM-liveness, $M$ has an execution of the form $\alpha \cdot \gamma$. By strict serializability of $M$, the execution fragment $\gamma$ must contain a nontrivial event on some base object that must be accessed by $\operatorname{read}_{n}(X)$ in $\sigma_{1} \cdots \sigma_{i} \cdot \sigma$. Suppose otherwise. Then, $\sigma_{1} \cdots \sigma_{i} \cdot \gamma \cdot \sigma$ is an execution of $M$ in which $\operatorname{read}_{n}(X) \rightarrow v$. But this execution is not strictly serializable: every transaction $T_{q} \in \operatorname{txns}(\alpha)$ must be aborted or must be preceded by $T_{n}$ in any serialization, but committed transaction $T_{n-1}$ must precede $T_{n}$ in any serialization to respect the real-time ordering of transactions. But then $\operatorname{read}_{n}(X)$ must return the new value $n v$ of $X$ that is updated by $T_{n-1}$ - contradiction.

Since, by Definition 2.18, the execution fragment $\gamma$ executed by some process $p_{n-1} \notin\left(\left\{p_{n}\right\} \cup S_{1} \cup \cdots \cup S_{i}\right)$ contains no nontrivial events to any base object accessed in $\sigma_{1} \cdots \sigma_{i}$, it must contain a nontrivial event to some base object $b_{i+1} \notin\left\{b_{1}, \ldots, b_{i}\right\}$ that is accessed by $T_{n}$ in the execution fragment $\sigma$.

Let $\mathcal{A}$ denote the set of all finite $\left(\left\{p_{n}\right\} \cup S_{1} \ldots \cup S_{i}\right)$-free execution fragments that extend $\alpha$. Let $b_{i+1} \notin\left\{b_{1}, \ldots, b_{i}\right\}$ be the first base object accessed by $T_{n}$ in the execution fragment $\sigma$ to which some transaction applies a nontrivial event in the execution fragment $\alpha^{\prime} \in \mathcal{A}$. Clearly, some such execution $\alpha \cdot \alpha^{\prime}$ exists that contains a nontrivial event in $\alpha^{\prime}$ to some distinct base object $b_{i+1}$ not accessed in the execution fragment $\sigma_{1} \cdots \sigma_{i}$. We choose the execution $\alpha \cdot \alpha^{\prime} \in \mathcal{A}$ that maximizes the number of transactions that are poised to apply nontrivial events on $b_{i+1}$ in the configuration after $\alpha \cdot \alpha^{\prime}$. Let $S_{i+1}$ denote the set of processes executing these transactions and $k^{\prime}=\left|S_{i+1}\right|\left(k^{\prime}>0\right.$ as already proved $)$.

We now construct a $\left(k+k^{\prime}\right)$-stall execution $\alpha \cdot \alpha^{\prime} \cdot \sigma_{1} \cdots \sigma_{i} \cdot \sigma_{i+1}$ for $\operatorname{read}_{n}(X)$, where in $\sigma_{i+1}, p_{n}$ applies events by itself, then each of the processes in $S_{i+1}$ applies a nontrivial event on $b_{i+1}$, and finally, $p_{n}$ accesses $b_{i+1}$.

By construction, $\alpha \cdot \alpha^{\prime}$ is $p_{n}$-free. Let $\sigma_{i+1}$ be the prefix of $\sigma$ not including $T_{n}$ 's first access to $b_{i+1}$, concatenated with the nontrivial events on $b_{i+1}$ by each of the $k^{\prime}$ transactions executed by processes in $S_{i+1}$ followed by the access of $b_{i+1}$ by $T_{n}$. Observe that $T_{n}$ performs exactly one t-operation $\operatorname{read}_{n}(X)$ in the execution fragment $\sigma_{1} \cdots \sigma_{i+1}$ and $\sigma_{1} \cdots \sigma_{i+1}$ contains no events of processes not in $\left(\left\{p_{n}\right\} \cup S_{1} \cup\right.$ $\left.\cdots \cup S_{i} \cup S_{i+1}\right)$.

To complete the induction, we need to show that in every $\left(\left\{p_{n}\right\} \cup S_{1} \cup \cdots \cup S_{i} \cup S_{i+1}\right)$-free extension of $\alpha \cdot \alpha^{\prime}$, no transaction applies a nontrivial event to any base object accessed in the execution fragment $\sigma_{1} \cdots \sigma_{i} \cdot \sigma_{i+1}$. Let $\beta$ be any such execution fragment that extends $\alpha \cdot \alpha^{\prime}$. By our construction, $\sigma_{i+1}$ is the execution fragment that consists of events by $p_{n}$ on base objects accessed in $\sigma_{1} \cdots \sigma_{i}$, nontrivial events on $b_{i+1}$ by transactions in $S_{i+1}$ and finally, an access to $b_{i+1}$ by $p_{n}$. Since $\alpha \cdot \sigma_{1} \cdots \sigma_{i}$ is a $k$-stall execution by our induction hypothesis, $\alpha^{\prime} \cdot \beta$ is $\left.\left(\left\{p_{n}\right\} \cup S_{1} \ldots \cup S_{i}\right\}\right)$-free and thus, $\alpha^{\prime} \cdot \beta$ does not contain nontrivial events on any base object accessed in $\sigma_{1} \cdots \sigma_{i}$. We now claim that $\beta$ does not contain nontrivial events to $b_{i+1}$. Suppose otherwise. Thus, there exists some transaction $T^{\prime}$ that has an enabled nontrivial event to $b_{i+1}$ in the configuration after $\alpha \cdot \alpha^{\prime} \cdot \beta^{\prime}$, where $\beta^{\prime}$ is some prefix of $\beta$. But this contradicts the choice of $\alpha \cdot \alpha^{\prime}$ as the extension of $\alpha$ that maximizes $k^{\prime}$.

Thus, $\alpha \cdot \alpha^{\prime} \cdot \sigma_{1} \cdots \sigma_{i} \cdot \sigma_{i+1}$ is indeed a $\left(k+k^{\prime}\right)$-stall execution for $T_{n}$ where $1<k<\left(k+k^{\prime}\right) \leq(n-1)$.

Since there are at most $n$ processes that are concurrent at any prefix of an execution, the lower bound of Theorem 5.3 is tight. 


\subsection{A linear lower bound on expensive synchronization for RW DAP}

We prove that opaque, RW DAP TM implementations in $\mathcal{O F}$ have executions in which some read-only transaction performs a linear (in $n$ ) number of non-overlapping RAWs or AWARs.

Prior to presenting the formal proof, we present an overview (the executions used in the proof are depicted in Figure 5.2.

We first construct an execution of the form $\bar{\rho}_{1} \cdots \bar{\rho}_{m}$, where for all $j \in\{1, \ldots, m\} ; m=n-3, \bar{\rho}_{j}$ denotes the t-complete step contention-free execution of transaction $T_{j}$ that reads the initial value $v$ in a distinct t-object $Z_{j}$, writes a new value $n v$ to a distinct t-object $X_{j}$ and commits. Observe that since any two transactions that participate in this execution are mutually read-write disjoint-access, they cannot contend on the same base object and, thus, the execution appears solo to each of them.

Let each of two new transactions $T_{n-1}$ and $T_{n}$ perform $m$ t-reads on objects $X_{1}, \ldots, X_{m}$. For $j \in$ $\{1, \ldots, m\}$, we now define $\rho_{j}$ to be the longest prefix of $\bar{\rho}_{j}$ such that $\rho_{1} \cdots \rho_{j}$ cannot be extended the complete step contention-free execution fragment of $T_{n-1}$ or $T_{n}$ where the t-read of $X_{j}$ returns $n v$ (Figure 5.2a). Let $e_{j}$ be the event by $T_{j}$ enabled after $\rho_{1} \cdots \rho_{j}$. Let us count the number of indices $j \in\{1, \ldots, m\}$ such that $T_{n-1}$ (resp., $T_{n}$ ) reads the new value $n v$ in $X_{j}$ when it runs after $\rho_{1} \cdots \rho_{j} \cdot e_{j}$. Without loss of generality, assume that $T_{n-1}$ has more such indices $j$ than $T_{n}$. We are going to show that, in the worst-case, $T_{n}$ must perform $\left\lceil\frac{m}{2}\right\rceil$ non-overlapping RAW/AWARs in the course of performing $m$ t-reads of $X_{1}, \ldots, X_{m}$ immediately after $\rho_{1} \cdots \rho_{m}$.

Consider any $j \in\{1, \ldots, m\}$ such that $T_{n-1}$, when it runs step contention-free after $\rho_{1} \cdots \rho_{j} \cdot e_{j}$, reads $n v$ in $X_{j}$. We claim that, in $\rho_{1} \cdots \rho_{m}$ extended with the step contention-free execution of $T_{n}$ performing $j$ t-reads $\operatorname{read}_{n}\left(X_{1}\right) \cdots \operatorname{read}_{n}\left(X_{j}\right)$, the t-read of $X_{j}$ must contain a RAW or an AWAR.

Suppose not. Then we are going to schedule a specific execution of $T_{j}$ and $T_{n-1}$ concurrently with read $_{n}\left(X_{j}\right)$ so that $T_{n}$ cannot detect the concurrency. By the definition of $\rho_{j}$ and the fact that the TM is RW DAP, $T_{n}$, when it runs step contention-free after $\rho_{1} \cdots \rho_{m}$, must read $v$ (the initial value) in $X_{j}$ (Figure 5.2b). Then the following execution exists: $\rho_{1} \cdots \rho_{m}$ is extended with the t-complete step contention-free execution of $T_{n-2}$ writing $n v$ to $Z_{j}$ and committing, after which $T_{n}$ runs step contentionfree and reads $v$ in $X_{j}$ (Figure 5.2c). Since, by the assumption, read $_{n}\left(X_{j}\right)$ contains no RAWs or AWARs, we show that we can run $T_{n-1}$ performing $j$ t-reads concurrently with the execution of $\operatorname{read}_{n}\left(X_{j}\right)$ so that $T_{n}$ and $T_{n-1}$ are unaware of step contention and read $_{n-1}\left(X_{j}\right)$ still reads the value $n v$ in $X_{j}$.

To understand why this is possible, consider the following: we take the execution depicted in Figure 5.2c but without the execution of $\operatorname{read}_{n}\left(X_{j}\right)$, i.e, $\rho_{1} \cdots \rho_{m}$ is extended with the step contention-free execution of committed transaction $T_{n-2}$ writing $n v$ to $Z_{j}$, after which $T_{n}$ runs step contention-free performing $j-1$ t-reads. This execution can be extended with the step $e_{j}$ by $T_{j}$, followed by the step contention-free execution of transaction $T_{n-1}$ in which it reads $n v$ in $X_{j}$. Indeed, by RW DAP and the definition of $\rho_{j} \cdot e_{j}$, there exists such an execution (Figure 5.2d).

Since read ${ }_{n}\left(X_{j}\right)$ contains no RAWs or AWARs, we can reschedule the execution fragment $e_{j}$ followed by the execution of $T_{n-1}$ so that it is concurrent with the execution of $\operatorname{read}_{n}\left(X_{j}\right)$ and neither $T_{n}$ nor $T_{n-1}$ see a difference (Figure 5.2e). Therefore, in this execution, $\operatorname{read}_{n}\left(X_{j}\right)$ still returns $v$, while $\operatorname{read}_{n-1}\left(X_{j}\right)$ returns $n v$.

However, the resulting execution (Figure 5.2e is not opaque. In any serialization the following must hold. Since $T_{n-1}$ reads the value written by $T_{j}$ in $X_{j}, T_{j}$ must be committed. Since $\operatorname{read}_{n}\left(X_{j}\right)$ returns the initial value $v, T_{n}$ must precede $T_{j}$. The committed transaction $T_{n-2}$, which writes a new value to $Z_{j}$, must precede $T_{n}$ to respect the real-time order on transactions. However, $T_{j}$ must precede $T_{n-2}$ since $\operatorname{read}_{j}\left(Z_{j}\right)$ returns the initial value and the implementation is opaque. The cycle $T_{j} \rightarrow T_{n-2} \rightarrow T_{n} \rightarrow T_{j}$ implies a contradiction.

Thus, we can show that transaction $T_{n}$ must perform $\Omega(n)$ RAW/AWARs during the execution of $m$ t-reads immediately after $\rho_{1} \cdots \rho_{m}$. 


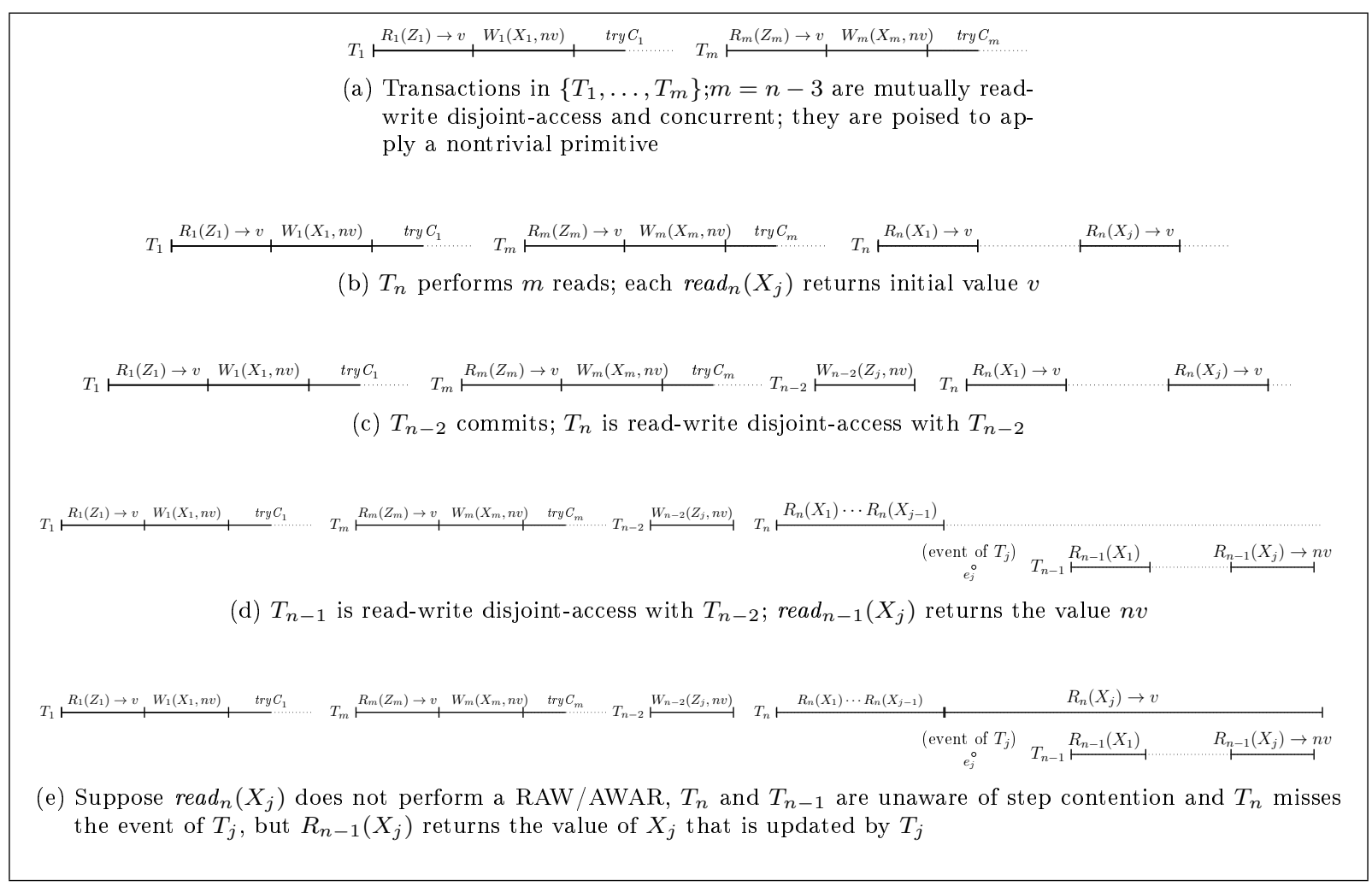

Figure 5.2: Executions in the proof of Theorem 5.4, execution in $5.2 \mathrm{e}$ is not opaque

Theorem 5.4. Every $R W$ DAP opaque TM implementation $M \in \mathcal{O F}$ has an execution $E$ in which some read-only transaction $T \in \operatorname{txns}(E)$ performs $\Omega(n)$ non-overlapping $R A W / A W A R$ s.

Proof. For all $j \in\{1, \ldots, m\} ; m=n-3$, let $v$ be the initial value of t-objects $X_{j}$ and $Z_{j}$. Throughout this proof, we assume that, for all $i \in\{1, \ldots, n\}$, transaction $T_{i}$ is invoked by process $p_{i}$.

By OF TM-progress and OF TM-liveness, any opaque and RW DAP TM implementation $M \in \mathcal{O F}$ has an execution of the form $\bar{\rho}_{1} \cdots \bar{\rho}_{m}$, where for all $j \in\{1, \ldots, m\}, \bar{\rho}_{j}$ denotes the t-complete step contention-free execution of transaction $T_{j}$ that performs $\operatorname{read}_{j}\left(Z_{j}\right) \rightarrow v$, writes value $n v \neq v$ to $X_{j}$ and commits.

By construction, any two transactions that participate in $\bar{\rho}_{1} \cdots \bar{\rho}_{n}$ are mutually read-write disjoint-access and cannot contend on the same base object. It follows that for all $1 \leq j \leq m, \bar{\rho}_{j}$ is an execution of $M$.

For all $j \in\{1, \ldots, m\}$, we iteratively define an execution $\rho_{j}$ of $M$ as follows: it is the longest prefix of $\bar{\rho}_{j}$ such that $\rho_{1} \cdots \rho_{j}$ cannot be extended with the complete step contention-free execution fragment of transaction $T_{n}$ that performs $j$ t-reads: $\operatorname{read}_{n}\left(X_{1}\right) \cdots \operatorname{read}_{n}\left(X_{j}\right)$ in which $\operatorname{read}_{n}\left(X_{j}\right) \rightarrow n v$ nor with the complete step contention-free execution fragment of transaction $T_{n-1}$ that performs $j$ t-reads: $\operatorname{read}_{n-1}\left(X_{1}\right) \cdots \operatorname{read}_{n-1}\left(X_{j}\right)$ in which $\operatorname{read}_{n-1}\left(X_{j}\right) \rightarrow n v$ (Figure 5.2a).

For any $j \in\{1, \ldots, m\}$, let $e_{j}$ be the event transaction $T_{j}$ is poised to apply in the configuration after $\rho_{1} \cdots \rho_{j}$. Thus, the execution $\rho_{1} \cdots \rho_{j} \cdot e_{j}$ can be extended with the complete step contention-free executions of at least one of transaction $T_{n}$ or $T_{n-1}$ that performs $j$ t-reads of $X_{1}, \ldots, X_{j}$ in which the t-read of $X_{j}$ returns the new value $n v$. Let $T_{n-1}$ be the transaction that must return the new value for the maximum number of $X_{j}$ 's when $\rho_{1} \cdots \rho_{j} \cdot e_{j}$ is extended with the t-reads of $X_{1}, \ldots, X_{j}$. We show that, in the worst-case, transaction $T_{n}$ must perform $\left\lceil\frac{m}{2}\right\rceil$ non-overlapping RAW/AWARs in the course of performing $m$ t-reads of $X_{1}, \ldots, X_{m}$ immediately after $\rho_{1} \cdots \rho_{m}$. Symmetric arguments apply for the case when $T_{n}$ must return the new value for the maximum number of $X_{j}$ 's when $\rho_{1} \cdots \rho_{j} \cdot e_{j}$ is extended with the t-reads of $X_{1}, \ldots, X_{j}$. 
Proving the RAW/AWAR lower bound. We prove that transaction $T_{n}$ must perform $\left\lceil\frac{m}{2}\right\rceil$ nonoverlapping RAWs or AWARs in the course of performing $m$ t-reads of $X_{1}, \ldots, X_{m}$ immediately after the execution $\rho_{1} \cdots \rho_{m}$. Specifically, we prove that $T_{n}$ must perform a RAW or an AWAR during the execution of the t-read of each $X_{j}$ such that $\rho_{1} \cdots \rho_{j} \cdot e_{j}$ can be extended with the complete step contention-free execution of $T_{n-1}$ as it performs $j$ t-reads of $X_{1} \ldots X_{j}$ in which the t-read of $X_{j}$ returns the new value $n v$. Let $\mathbb{J}$ denote the of all $j \in\{1, \ldots, m\}$ such that $\rho_{1} \cdots \rho_{j} \cdot e_{j}$ extended with the complete step contention-free execution of $T_{n-1}$ performing $j$ t-reads of $X_{1} \ldots X_{j}$ must return the new value $n v$ during the t-read of $X_{j}$.

We first prove that, for all $j \in \mathbb{J}, M$ has an execution of the form $\rho_{1} \cdots \rho_{m} \cdot \delta_{j}$ (Figures $5.2 \mathrm{a}$ and $5.2 \mathrm{~b}$, where $\delta_{j}$ is the complete step contention-free execution fragment of $T_{n}$ that performs $j$ t-reads: $\operatorname{read}_{n}\left(X_{1}\right) \cdots \operatorname{read}_{n}\left(X_{j}\right)$, each of which return the initial value $v$.

By definition of $\rho_{j}$, OF TM-progress and OF TM-liveness, $M$ has an execution of the form $\rho_{1} \cdots \rho_{j} \cdot \delta_{j}$. By construction, transaction $T_{n}$ is read-write disjoint-access with each transaction $T \in\left\{T_{j+1}, \ldots, T_{m}\right\}$ in $\rho_{1} \cdots \rho_{j} \cdots \rho_{m} \cdot \delta_{j}$. Thus, $T_{n}$ cannot contend with any of the transactions in $\left\{T_{j+1}, \ldots, T_{m}\right\}$, implying that, for all $j \in\{1, \ldots, m\}, M$ has an execution of the form $\rho_{1} \cdots \rho_{m} \cdot \delta_{j}$ (Figure $5.2 \mathrm{~b}$ ).

We claim that, for each $j \in \mathbb{J}$, the t-read of $X_{j}$ performed by $T_{n}$ must perform a RAW or an AWAR in the course of performing $j$ t-reads of $X_{1}, \ldots, X_{j}$ immediately after $\rho_{1} \cdots \rho_{m}$. Suppose by contradiction that $\operatorname{read}_{n}\left(X_{j}\right)$ does not perform a RAW or an AWAR in $\rho_{1} \cdots \rho_{m} \cdot \delta_{m}$.

Claim 5.5. For all $j \in \mathbb{J}, M$ has an execution of the form $\rho_{1} \cdots \rho_{j} \cdots \rho_{m} \cdot \delta_{j-1} \cdot e_{j} \cdot \beta$ where, $\beta$ is the complete step contention-free execution fragment of transaction $T_{n-1}$ that performs $j$ t-reads: $\operatorname{read}_{n-1}\left(X_{1}\right) \cdots \operatorname{read}_{n-1}\left(X_{j-1}\right) \cdot \operatorname{read}_{n-1}\left(X_{j}\right)$ in which read $n-1\left(X_{j}\right)$ returns $n v$.

Proof. We observe that transaction $T_{n}$ is read-write disjoint-access with every transaction $T \in\left\{T_{j}, T_{j+1}, \ldots, T_{m}\right\}$ in $\rho_{1} \cdots \rho_{j} \cdots \rho_{m} \cdot \delta_{j-1}$. By RW DAP, it follows that $M$ has an execution of the form $\rho_{1} \cdots \rho_{j} \cdots \rho_{m} \cdot \delta_{j-1} \cdot e_{j}$ since $T_{n}$ cannot perform a nontrivial event on the base object accessed by $T_{j}$ in the event $e_{j}$.

By the definition of $\rho_{j}$, transaction $T_{n-1}$ must access the base object to which $T_{j}$ applies a nontrivial primitive in $e_{j}$ to return the value $n v$ of $X_{j}$ as it performs $j$ t-reads of $X_{1}, \ldots, X_{j}$ immediately after the execution $\rho_{1} \cdots \rho_{j} \cdots \rho_{m} \cdot \delta_{j-1} \cdot e_{j}$. Thus, $M$ has an execution of the form $\rho_{1} \cdots \rho_{j} \cdot \delta_{j-1} \cdot e_{j} \cdot \beta$.

By construction, transactions $T_{n-1}$ is read-write disjoint-access with every transaction $T \in\left\{T_{j+1}, \ldots, T_{m}\right\}$ in $\rho_{1} \cdots \rho_{j} \cdots \rho_{m} \cdot \delta_{j-1} \cdot e_{j} \cdot \beta$. It follows that $M$ has an execution of the form $\rho_{1} \cdots \rho_{j} \cdots \rho_{m} \cdot \delta_{j-1} \cdot e_{j} \cdot \beta$.

Claim 5.6. For all $j \in\{1, \ldots, m\}, M$ has an execution of the form $\rho_{1} \cdots \rho_{j} \cdots \rho_{m} \cdot \gamma \cdot \delta_{j-1} \cdot e_{j} \cdot \beta$, where $\gamma$ is the t-complete step contention-free execution fragment of transaction $T_{n-2}$ that writes nv $\neq v$ to $Z_{j}$ and commits.

Proof. Observe that $T_{n-2}$ precedes transactions $T_{n}$ and $T_{n-1}$ in real-time order in the above execution.

By OF TM-progress and OF TM-liveness, transaction $T_{n-2}$ must be committed in $\rho_{1} \cdots \rho_{j} \cdots \rho_{m} \cdot \gamma$.

Since transaction $T_{n-1}$ is read-write disjoint-access with $T_{n-2}$ in $\rho_{1} \cdots \rho_{j} \cdots \rho_{m} \cdot \gamma \cdot \delta_{j-1} \cdot e_{j} \cdot \beta, T_{n-1}$ does not contend with $T_{n-2}$ on any base object (recall that we associate an edge with t-objects in the conflict graph only if they are both contained in the write set of some transaction). Since the execution fragment $\beta$ contains an access to the base object to which $T_{j}$ performs a nontrivial primitive in the event $e_{j}, T_{n-2}$ cannot perform a nontrivial event on this base object in $\gamma$. It follows that $M$ has an execution of the form $\rho_{1} \cdots \rho_{j} \cdots \rho_{m} \cdot \gamma \cdot \delta_{j-1} \cdot e_{j} \cdot \beta$ since, it is indistinguishable to $T_{n-1}$ from the execution $\rho_{1} \cdots \rho_{j} \cdots \rho_{m} \cdot \delta_{j-1} \cdot e_{j} \cdot \beta$ (the existence of which is already established in Claim 5.5).

Recall that transaction $T_{n}$ is read-write disjoint-access with $T_{n-2}$ in $\rho_{1} \cdots \rho_{j} \cdots \rho_{m} \cdot \gamma \cdot \delta_{j}$. Thus, $M$ has an execution of the form $\rho_{1} \cdots \rho_{j} \cdots \rho_{m} \cdot \gamma \cdot \delta_{j}$ (Figure 5.2c).

Deriving a contradiction. For all $j \in\{1, \ldots, m\}$, we represent the execution fragment $\delta_{j}$ as $\delta_{j-1} \cdot \pi^{j}$, where $\pi^{j}$ is the complete execution fragment of the $j^{\text {th }}$ t-read $\operatorname{read}_{n}\left(X_{j}\right) \rightarrow v$. By our assumption, $\pi^{j}$ does not contain a RAW or an AWAR. 
For succinctness, let $\alpha=\rho_{1} \cdots \rho_{m} \cdot \gamma \cdot \delta_{j-1}$. We now prove that if $\pi^{j}$ does not contain a RAW or an AWAR, we can define $\pi_{1}^{j} \cdot \pi_{2}^{j}=\pi^{j}$ to construct an execution of the form $\alpha \cdot \pi_{1}^{j} \cdot e_{j} \cdot \beta \cdot \pi_{2}^{j}$ (Figure 5.2e such that

- no event in $\pi_{1}^{j}$ is the application of a nontrivial primitive

- $\alpha \cdot \pi_{1}^{j} \cdot e_{j} \cdot \beta \cdot \pi_{2}^{j}$ is indistinguishable to $T_{n}$ from the step contention-free execution $\alpha \cdot \pi_{1}^{j} \cdot \pi_{2}^{j}$

- $\alpha \cdot \pi_{1}^{j} \cdot e_{j} \cdot \beta \cdot \pi_{2}^{j}$ is indistinguishable to $T_{n-1}$ from the step contention-free execution $\alpha \cdot e_{j} \cdot \beta$.

The following claim defines $\pi_{1}^{j}$ and $\pi_{2}^{j}$ to construct this execution.

Claim 5.7. For all $j \in\{1, \ldots, m\}, M$ has an execution of the form $\alpha \cdot \pi_{1}^{j} \cdot e_{j} \cdot \beta \cdot \pi_{2}^{j}$.

Proof. Let $t$ be the first event containing a write to a base object in the execution fragment $\pi^{j}$. We represent $\pi^{j}$ as the execution fragment $\pi_{1}^{j} \cdot t \cdot \pi_{f}^{j}$. Since $\pi_{1}^{j}$ does not contain nontrivial events that write to a base object, $\alpha \cdot \pi_{1}^{j} \cdot e_{j} \cdot \beta$ is indistinguishable to transaction $T_{n-1}$ from the step contention-free execution $\alpha \cdot e_{j} \cdot \beta$ (as already proven in Claim 5.6. Consequently, $\alpha \cdot \pi_{1}^{j} \cdot e_{j} \cdot \beta$ is an execution of $M$.

Since $t$ is not an atomic-write-after-read, $M$ has an execution of the form $\alpha \cdot \gamma \cdot \pi_{1}^{j} \cdot e_{j} \cdot \beta \cdot t$. Secondly, since $\pi^{j}$ does not contain a read-after-write, any read of a base object performed in $\pi_{f}^{j}$ may only be performed to base objects previously written in $t \cdot \pi_{f}^{j}$. Thus, $\alpha \cdot \pi_{1}^{j} \cdot e_{j} \cdot \beta \cdot t \cdot \pi_{f}^{j}$ is indistinguishable to $T_{n}$ from the step contention-free execution $\alpha \cdot \pi_{1}^{j} \cdot t \cdot \pi_{f}^{j}$. But, as already proved, $\alpha \cdot \pi^{j}$ is an execution of $M$.

Choosing $\pi_{2}^{j}=t \cdot \pi_{f}^{j}$, it follows that $M$ has an execution of the form $\alpha \cdot \pi_{1}^{j} \cdot e_{j} \cdot \beta \cdot \pi_{2}^{j}$.

We have now proved that, for all $j \in\{1, \ldots, m\}, M$ has an execution of the form $\rho_{1} \cdots \rho_{m} \cdot \gamma \cdot \delta_{j-1} \cdot \pi_{1}^{j}$. $e_{j} \cdot \beta \cdot \pi_{2}^{j}$ (Figure $5.2 \mathrm{e}$ ).

The execution in Figure $5.2 \mathrm{e}$ is not opaque. Indeed, in any serialization the following must hold. Since $T_{n-1}$ reads the value written by $T_{j}$ in $X_{j}, T_{j}$ must be committed. Since read $d_{n}\left(X_{j}\right)$ returns the initial value $v, T_{n}$ must precede $T_{j}$. The committed transaction $T_{n-2}$, which writes a new value to $Z_{j}$, must precede $T_{n}$ to respect the real-time order on transactions. However, $T_{j}$ must precede $T_{n-2}$ since $\operatorname{read}_{j}\left(Z_{j}\right)$ returns the initial value of $Z_{j}$. The cycle $T_{j} \rightarrow T_{n-2} \rightarrow T_{n} \rightarrow T_{j}$ implies that there exists no such a serialization.

Thus, for each $j \in \mathbb{J}$, transaction $T_{n}$ must perform a RAW or an AWAR during the t-read of $X_{j}$ in the course of performing $m$ t-reads of $X_{1}, \ldots, X_{m}$ immediately after $\rho_{1} \cdots \rho_{m}$. Since $|\mathbb{J}| \geq\left\lceil\frac{(n-3)}{2}\right\rceil$, in the worst-case, $T_{n}$ must perform $\Omega(n)$ RAW/AWARs during the execution of $m$ t-reads immediately after $\rho_{1} \cdots \rho_{m}$.

\subsection{Algorithms for obstruction-free TMs}

In this section, we present two opaque obstruction-free TM implementations: the first one satisfies RW DAP, but not strict DAP while the second one satisfies weak DAP, but not RW DAP. 


\subsubsection{An opaque RW DAP TM implementation}

In this section, we describe a RW DAP TM implementation in $\mathcal{O F}$ (based on DSTM [77]).

Every t-object $X_{m}$ maintains a base object $\operatorname{tvar}[m]$ and every transaction $T_{k}$ maintains a status $[k]$ base object. Both base objects support the read, write and compare-and-swap (cas) primitives.

The object $\operatorname{tvar}[m]$ stores a triple: the owner of $X_{m}$ is an updating transaction that performs the latest write to $X_{m}$, the old value and new value of $X_{m}$ represent two latest versions of $X_{m}$. The base object status $[k]$ denotes if $T_{k}$ is live (i.e. t-incomplete), committed or aborted. Intuitively, if status $[k]$ is committed, then other transactions can safely read the value of the t-objects updated by $T_{k}$.

Implementation of $\operatorname{read}_{k}\left(X_{m}\right)$ first reads tvar $[m]$ and checks if the owner of $X_{m}$ is live; if so, it forcefully aborts the owning transaction and returns the old value of $X_{m}$. Otherwise, if the owner is committed, it returns the new value of $X_{m}$. In both cases, it only returns a non-abort value if no t-object previously read has been updated since. The write $_{k}\left(X_{m}, v\right)$ works similar to the $\operatorname{read}_{k}\left(X_{m}\right)$ implementation; but additionally, if the owner of $X_{m}$ is live, it forcefully aborts the owning transaction, assumes ownership of $X_{m}$, sets $v$ as the new value of $X_{m}$ and leaves the old value of $X_{m}$ unchanged. Otherwise, if the owner of $X_{m}$ is a committed transaction, it updates the old value of $X_{m}$ to be the value of $X_{m}$ updated by its previous owner. The try $C_{k}$ implementation sets status $[k]$ to committed if it has not been set to aborted by a concurrent transaction, otherwise $T_{k}$ is deemed aborted. Since any t-read operation performs at most two AWARs and the try $C$ performs only a single AWAR, any read-only transaction $T$ performs at most $O(|R \operatorname{set}(T)|)$ AWARs. The pseudocode is described in Algorithm 5.1.

Lemma 5.8. Algorithm 5.1 implements an opaque TM.

Proof. Since opacity is a safety property, we only consider finite executions [17]. Let $E$ by any finite execution of Algorithm 5.1. Let $<_{E}$ denote a total-order on events in $E$.

Let $H$ denote a subsequence of $E$ constructed by selecting linearization points of t-operations performed in $E$. The linearization point of a t-operation $o p$, denoted as $\ell_{o p}$ is associated with a base object event or an event performed during the execution of $o p$ using the following procedure.

Completions. First, we obtain a completion of $E$ by removing some pending invocations and adding responses to the remaining pending invocations involving a transaction $T_{k}$ as follows: every incomplete read $_{k}$, write $e_{k}$, try $_{k}$ operation is removed from $E$; an incomplete write $e_{k}$ is removed from $E$.

Linearization points. We now associate linearization points to t-operations in the obtained completion of $E$ as follows:

- For every t-read $o p_{k}$ that returns a non- $\mathrm{A}_{k}$ value, $\ell_{o p_{k}}$ is chosen as the event in Line 12 of Algorithm 5.1, else, $\ell_{o p_{k}}$ is chosen as invocation event of $o p_{k}$

- For every t-write $o p_{k}$ that returns a non- $\mathrm{A}_{k}$ value, $\ell_{o p_{k}}$ is chosen as the event in Line 37 of Algorithm 5.1, else, $\ell_{o p_{k}}$ is chosen as invocation event of $o p_{k}$

- For every $o p_{k}=\operatorname{try} C_{k}$ that returns $C_{k}, \ell_{o p_{k}}$ is associated with Line 65

$<_{H}$ denotes a total-order on t-operations in the complete sequential history $H$.

Serialization points. The serialization of a transaction $T_{j}$, denoted as $\delta_{T_{j}}$ is associated with the linearization point of a t-operation performed during the execution of the transaction.

We obtain a t-complete history $\bar{H}$ from $H$ as follows: for every transaction $T_{k}$ in $H$ that is complete, but not t-complete, we insert $\operatorname{try} C_{k} \cdot A_{k}$ after $H$.

$\bar{H}$ is thus a t-complete sequential history. A t-complete t-sequential history $S$ equivalent to $\bar{H}$ is obtained by associating serialization points to transactions in $\bar{H}$ as follows:

- If $T_{k}$ is an update transaction that commits, then $\delta_{T_{k}}$ is $\ell_{t r y C_{k}}$ 


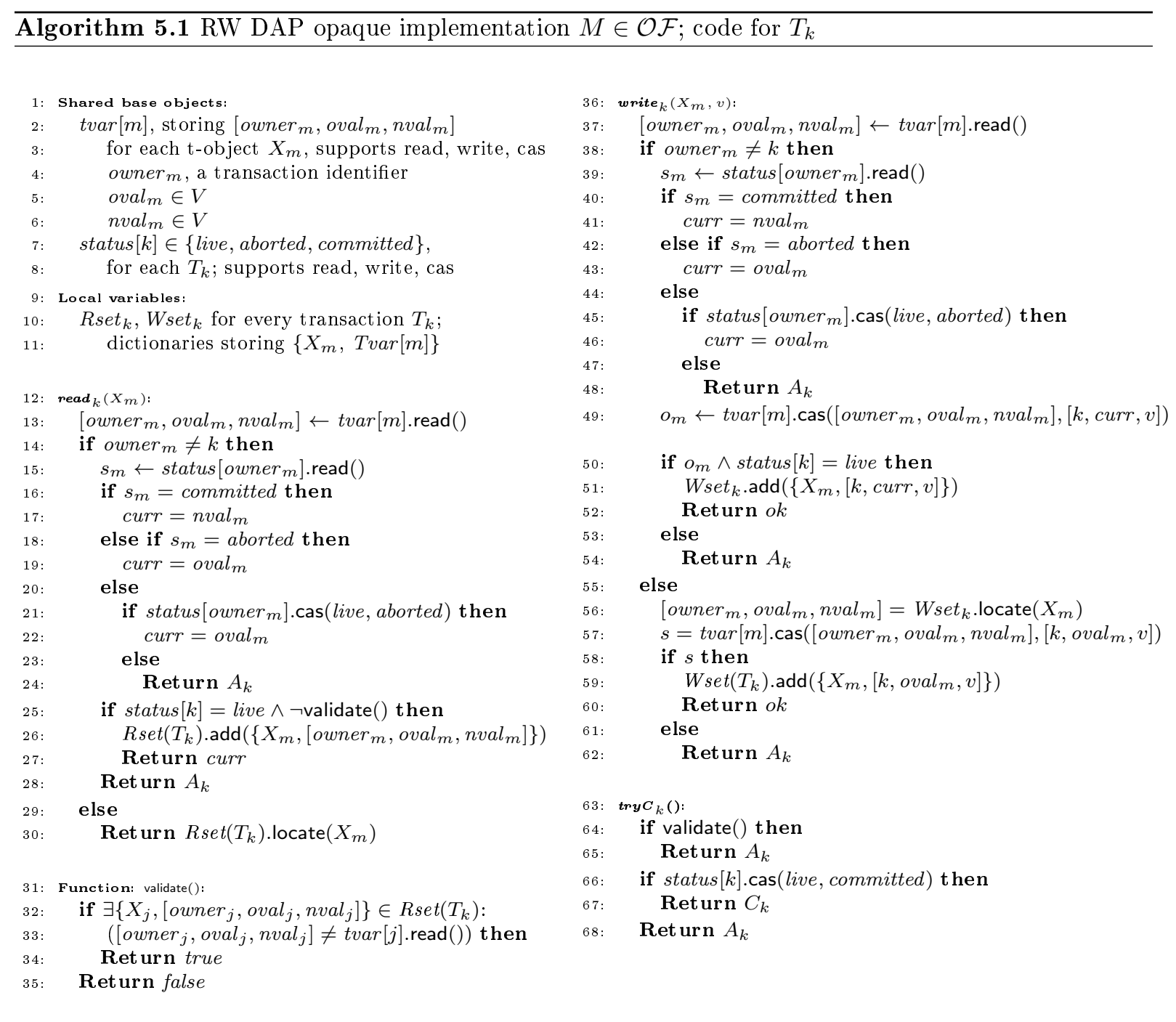

- If $T_{k}$ is an aborted or read-only transaction in $\bar{H}$, then $\delta_{T_{k}}$ is assigned to the linearization point of the last t-read that returned a non- $\mathrm{A}_{k}$ value in $T_{k}$

$<_{S}$ denotes a total-order on transactions in the t-sequential history $S$.

Claim 5.9. If $T_{i} \prec_{H}^{R T} T_{j}$, then $T_{i}<_{S} T_{j}$.

Proof. This follows from the fact that for a given transaction, its serialization point is chosen between the first and last event of the transaction implying if $T_{i} \prec_{H} T_{j}$, then $\delta_{T_{i}}<_{E} \delta_{T_{j}}$ implies $T_{i}<_{S} T_{j}$

Claim 5.10. If transaction $T_{i}$ returns $C_{i}$ in $E$, then status[i] $=$ committed in $E$.

Proof. Transaction $T_{i}$ must perform the event in Line 66 before returning $T_{i}$ i.e. the cas on its own status to change the value to committed. The proof now follows from the fact that any other transaction may change the status of $T_{i}$ only if it is live (Lines 45 and 21).

Claim 5.11. $S$ is legal.

Proof. Observe that for every $\operatorname{read}_{j}(X) \rightarrow v$, there exists some transaction $T_{i}$ that performs write $_{i}(X, v)$ and completes the event in Line 22 to write $v$ as the new value of $X$ such that read ${ }_{j}(X) \nprec_{H}^{R T}$ write $_{i}(X, v)$. For any updating committing transaction $T_{i}, \delta_{T_{i}}=\ell_{\text {try } C_{i}}$. Since $\operatorname{read}_{j}(X)$ returns a response $v$, the event 
in Line 12 must succeed the event in Line 66 when $T_{i}$ changes status $[i]$ to committed. Suppose otherwise, then read $_{j}(X)$ subsequently forces $T_{i}$ to abort by writing aborted to status [i] and must return the old value of $X$ that is updated by the previous owner of $X$, which must be committed in $E$ (Line 40 ). Since $\delta_{T_{i}}=\ell_{\text {try } C_{i}}$ precedes the event in Line 66, it follows that $\delta_{T_{i}}<_{E} \ell_{\text {read }_{j}(X)}$.

We now need to prove that $\delta_{T_{i}}<_{E} \delta_{T_{j}}$. Consider the following cases:

- if $T_{j}$ is an updating committed transaction, then $\delta_{T_{j}}$ is assigned to $\ell_{\text {try }_{j}}$. But since $\ell_{\text {read }_{j}(X)}<_{E}$ $\ell_{t r y C_{j}}$, it follows that $\delta_{T_{i}}<_{E} \delta_{T_{j}}$.

- if $T_{j}$ is a read-only or aborted transaction, then $\delta_{T_{j}}$ is assigned to the last t-read that did not abort. Again, it follows that $\delta_{T_{i}}<_{E} \delta_{T_{j}}$.

To prove that $S$ is legal, we need to show that, there does not exist any transaction $T_{k}$ that returns $C_{k}$ in $S$ and performs write $e_{k}\left(X, v^{\prime}\right) ; v^{\prime} \neq v$ such that $T_{i}<_{S} T_{k}<_{S} T_{j}$. Now, suppose by contradiction that there exists a committed transaction $T_{k}, X \in W \operatorname{set}\left(T_{k}\right)$ that writes $v^{\prime} \neq v$ to $X$ such that $T_{i}<_{S} T_{k}<_{S} T_{j}$. Since $T_{i}$ and $T_{k}$ are both updating transactions that commit,

$$
\begin{gathered}
\left(T_{i}<_{S} T_{k}\right) \Longleftrightarrow\left(\delta_{T_{i}}<_{E} \delta_{T_{k}}\right) \\
\left(\delta_{T_{i}}<_{E} \delta_{T_{k}}\right) \Longleftrightarrow\left(\ell_{t r y C_{i}}<_{E} \ell_{t r y} C_{k}\right)
\end{gathered}
$$

Since, $T_{j}$ reads the value of $X$ written by $T_{i}$, one of the following is true: $\ell_{\operatorname{try} C_{i}}<_{E} \ell_{t r y C_{k}}<_{E} \ell_{\text {read }_{j}(X)}$ or $\ell_{\text {try } C_{i}}<_{E} \ell_{\text {read }_{j}(X)}<_{E} \ell_{\text {try }} C_{k}$.

If $\ell_{t r y C_{i}}<_{E} \ell_{t r y C_{k}}<_{E} \ell_{\text {read }_{j}(X)}$, then the event in Line 66 performed by $T_{k}$ when it changes the status field to committed precedes the event in Line 12 performed by $T_{j}$. Since $\ell_{t r y C_{i}}<_{E} \ell_{t r y} C_{k}$ and both $T_{i}$ and $T_{k}$ are committed in $E, T_{k}$ must perform the event in Line 37 after $T_{i}$ changes status $[i]$ to committed since otherwise, $T_{k}$ would perform the event in Line 45 and change status/i] to aborted, thereby forcing $T_{i}$ to return $A_{i}$. However, $\operatorname{read}_{j}(X)$ observes that the owner of $X$ is $T_{k}$ and since the status of $T_{k}$ is committed at this point in the execution, $\operatorname{read}_{j}(X)$ must return $v^{\prime}$ and not $v$-contradiction.

Thus, $\ell_{t r y C_{i}}<_{E} \ell_{\text {read }_{j}(X)}<_{E} \ell_{t r y C_{k}}$. We now need to prove that $\delta_{T_{j}}$ indeed precedes $\delta_{T_{k}}=\ell_{\text {try } C_{k}}$ in $E$.

Now consider two cases:

- Suppose that $T_{j}$ is a read-only transaction. Then, $\delta_{T_{j}}$ is assigned to the last t-read performed by $T_{j}$ that returns a non- $\mathrm{A}_{j}$ value. If $\operatorname{read}_{j}(X)$ is not the last t-read that returned a non- $\mathrm{A}_{j}$ value, then there exists a $\operatorname{read}_{j}\left(X^{\prime}\right)$ such that $\ell_{\text {read }_{j}(X)}<_{E} \ell_{t r y C_{k}}<_{E} \ell_{\text {read }_{j}\left(X^{\prime}\right)}$. But then this t-read of $X^{\prime}$ must abort since the value of $X$ has been updated by $T_{k}$ since $T_{j}$ first read $X$-contradiction.

- Suppose that $T_{j}$ is an updating transaction that commits, then $\delta_{T_{j}}=\ell_{\text {try }}$, which implies that $\ell_{\text {read }_{j}(X)}<_{E} \ell_{t r y C_{k}}<_{E} \ell_{t r y C_{j}}$. Then, $T_{j}$ must neccesarily perform the validation of its read set in Line 65 and return $A_{j}$-contradiction.

Claims 5.9 and 5.11 establish that Algorithm 5.1 is opaque.

Theorem 5.12. Algorithm 5.1 describes a $R W D A P$, progressive opaque TM implementation $M \in \mathcal{O F}$ such that in every execution $E$ of $M$,

- the total number of stalls incurred by a t-read operation invoked in $E$ is $O(n)$,

- every read-only transaction $T \in \operatorname{txns}(E)$ performs $O(|R \operatorname{set}(T)|)$ AWARs in $E$, and

- every complete t-read operation invoked by transaction $T_{k} \in \operatorname{txns}(E)$ performs $O\left(\left|R_{s e t}\left(T_{k}\right)\right|\right.$ steps. 


\section{Proof. (Opacity) Follows from Lemma 5.8}

(TM-liveness and TM-progress) Since none of the implementations of the t-operations in Algorithm 5.1 contain unbounded loops or waiting statements, every t-operation $o p_{k}$ returns a matching response after taking a finite number of steps. Thus, Algorithm 5.1 provides wait-free TM-liveness.

To prove OF TM-progress, we proceed by enumerating the cases under which a transaction $T_{k}$ may be aborted in any execution.

- Suppose that there exists a $\operatorname{read}_{k}\left(X_{m}\right)$ performed by $T_{k}$ that returns $A_{k}$. If $\operatorname{read}_{k}\left(X_{m}\right)$ returns $A_{k}$ in Line 28 then there exists a concurrent transaction that updated a t-object in $\operatorname{Rset}\left(T_{k}\right)$ or changed status $[k]$ to aborted. In both cases, $T_{k}$ returns $A_{k}$ only because there is step contention.

- Suppose that there exists a write $e_{k}\left(X_{m}, v\right)$ performed by $T_{k}$ that returns $A_{k}$ in Line 54 Thus, either a concurrent transaction has changed status $[k]$ to aborted or the value in $\operatorname{tvar}[m]$ has been updated since the event in Line 37 . In both cases, $T_{k}$ returns $A_{k}$ only because of step contention with another transaction.

- Suppose that a $\operatorname{read}_{k}\left(X_{m}\right)$ or write $_{k}\left(X_{m}, v\right)$ return $A_{k}$ in Lines 21 and 45 respectively. Thus, a concurrent transaction has takes steps concurrently by updating the status of owner $m$ since the read by $T_{k}$ in Lines 12 and 37 respectively.

- Suppose that $\operatorname{try} C_{k}()$ returns $A_{k}$ in Line62. This is because there exists a t-object in $\operatorname{Rset}\left(T_{k}\right)$ that has been updated by a concurrent transaction since, i.e., $\operatorname{try} C_{k}()$ returns $A_{k}$ only on encountering step contention.

It follows that in any step contention-free execution of a transaction $T_{k}$ from a $T_{k}$-free execution, $T_{k}$ must return $C_{k}$ after taking a finite number of steps.

The enumeration above also proves that $M$ implements a progressive TM.

(Read-write disjoint-access parallelism) Consider any execution $E$ of Algorithm 5.1 and let $T_{i}$ and $T_{j}$ be any two transactions that contend on a base object $b$ in $E$. We need to prove that there is a path between a t-object in $\operatorname{Dset}\left(T_{i}\right)$ and a t-object in $\operatorname{Dset}\left(T_{j}\right)$ in $\tilde{G}\left(T_{i}, T_{j}, E\right)$ or there exists $X \in$ $\operatorname{Dset}\left(T_{i}\right) \cap \operatorname{Dset}\left(T_{j}\right)$. Recall that there exists an edge between t-objects $X$ and $Y$ in $\tilde{G}\left(T_{i}, T_{j}, E\right)$ only if there exists a transaction $T \in \operatorname{txns}(E)$ such that $\{X, Y\} \in \operatorname{Wset}(T)$.

- Suppose that $T_{i}$ and $T_{j}$ contend on base object $\operatorname{tvar}[\mathrm{m}]$ belonging to t-object $X_{m}$ in $E$. By Algorithm 5.1, a transaction accesses $X_{m}$ only if $X_{m}$ is contained in $\operatorname{Dset}\left(T_{m}\right)$. Thus, both $T_{i}$ and $T_{j}$ must access $X_{m}$.

- Suppose that $T_{i}$ and $T_{j}$ contend on base object status $[\mathrm{i}]$ in $E$ (the case when $T_{i}$ and $T_{j}$ contend on status $[\mathrm{j}]$ is symmetric). $T_{j}$ accesses status $[i]$ while performing a t-read of some t-object $X$ in Lines 15 and 21 only if $T_{i}$ is the owner of $X$. Also, $T_{j}$ accesses status[i] while performing a t-write to $X$ in Lines 39 and 45 only if $T_{i}$ is the owner of $X$. But if $T_{i}$ is the owner of $X$, then $X \in W \operatorname{set}\left(T_{i}\right)$.

- Suppose that $T_{i}$ and $T_{j}$ contend on base object status $[\mathrm{m}]$ belonging to some transaction $T_{m}$ in $E$. Firstly, observe that $T_{i}$ or $T_{j}$ access status $\left./ m\right]$ only if there exist t-objects $X$ and $Y$ in $\operatorname{Dset}\left(T_{i}\right)$ and $\operatorname{Dset}\left(T_{j}\right)$ respectively such that $\{X, Y\} \in W \operatorname{wet}\left(T_{m}\right)$. This is because $T_{i}$ and $T_{j}$ would both read status $/ \mathrm{m} /$ in Lines 15 (during t-read) and 39 (during t-write) only if $T_{m}$ was the previous owner of $X$ and $Y$. Secondly, one of $T_{i}$ or $T_{j}$ applies a nontrivial primitive to status $[\mathrm{m}]$ only if $T_{i}$ and $T_{j}$ read status $[m]=$ live in Lines 15 (during t-read) and 37 (during t-write). Thus, at least one of $T_{i}$ or $T_{j}$ is concurrent to $T_{m}$ in $E$. It follows that there exists a path between $X$ and $Y$ in $\tilde{G}\left(T_{i}, T_{j}, E\right)$.

(Complexity) Every t-read operation performs at most one AWAR in an execution $E$ (Line 21) of Algorithm 5.1. It follows that any read-only transaction $T_{k} \in \operatorname{txns}(E)$ performs at most $\left|R \operatorname{set}\left(T_{k}\right)\right|$ AWARs in $E$.

The linear step-complexity is immediate from the fact that during the t-read operations, the transaction validates its entire read set (Line 25). All other t-operations incur $O(1)$ step-complexity since they involve no iteration statements like for and while loops. 
Since at most $n-1$ transactions may be t-incomplete at any point in an execution $E$, it follows that $E$ is at most a $(n-1)$-stall execution for any t-read op and every $T \in \operatorname{txns}(E)$ incurs $O(n)$ stalls on account of any event performed in $E$. More specifically, consider the following execution $E$ : for all $i \in\{1, \ldots, n-1\}$, each transaction $T_{i}$ performs write $_{i}\left(X_{m}, v\right)$ in a step-contention free execution until it is poised to apply a nontrivial event on tvar $[m]$ (Line 22). By OF TM-progress, we construct $E$ such that each of the $T_{i}$ is poised to apply a nontrivial event on $\operatorname{tvar}[m]$ after $E$. Consider the execution fragment of $\operatorname{read}_{n}\left(X_{m}\right)$ that is poised to perform an event $e$ that reads tvar $[m]$ (Line 12 immediately after $E$. In the constructed execution, $T_{n}$ incurs $O(n)$ stalls on account of $e$ and thus, produces the desired $(n-1)$-stall execution for $\operatorname{read}_{n}(X)$.

\subsubsection{An opaque weak DAP TM implementation}

In this section, we describe a weak DAP TM implementation in $\mathcal{O} \mathcal{F}$ with constant step-complexity t-read operations.

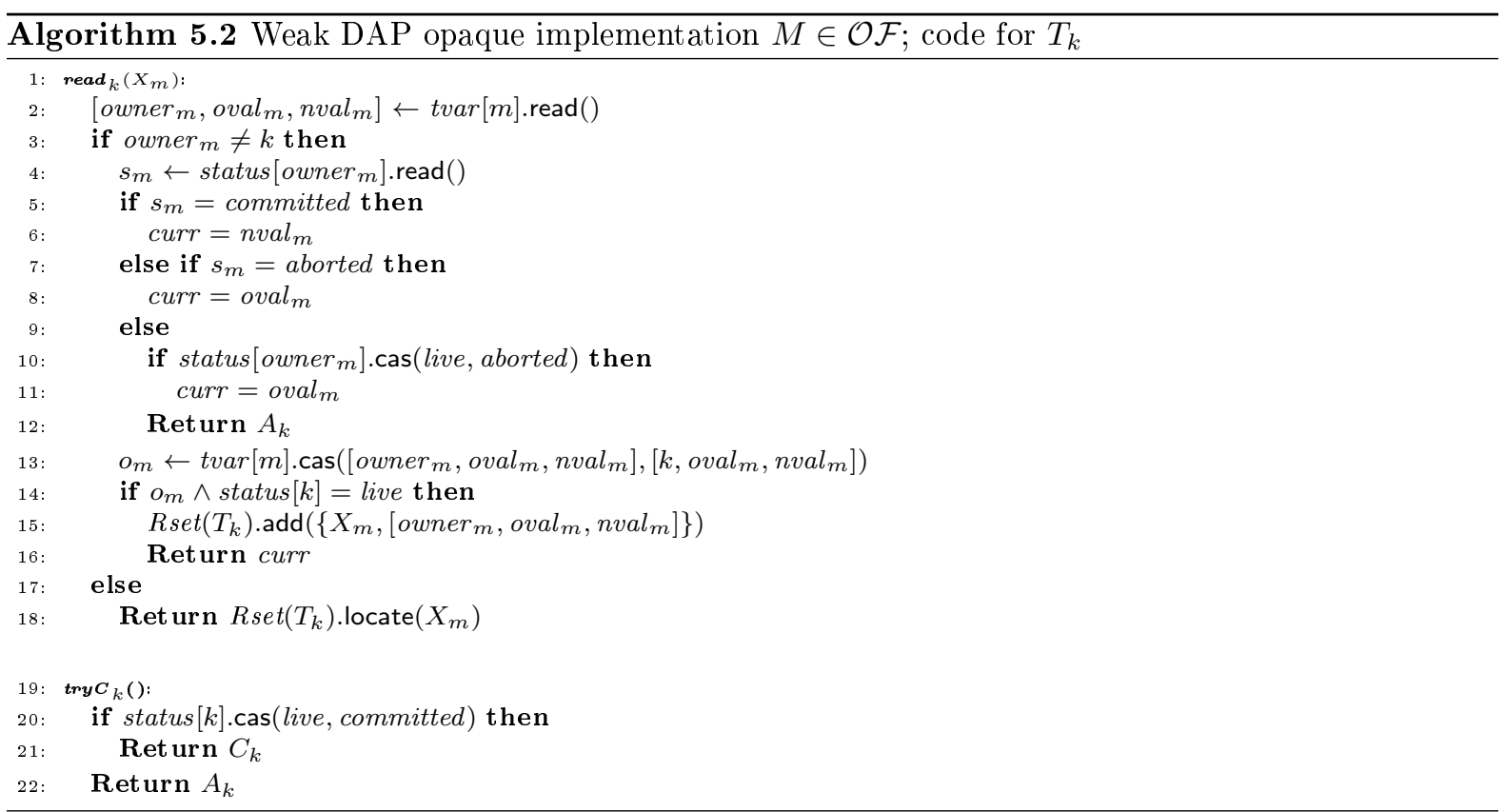

Algorithm 5.2 describes a weak DAP implementation in $\mathcal{O F}$ that does not satisfy read-write DAP. The code for the t-write operations is identical to Algorithm 5.1. During the t-read of t-object $X_{m}$ by transaction $T_{k}, T_{k}$ becomes the owner of $X_{m}$ thus eliminating the per-read validation step-complexity inherent to Algorithm 5.1. Similarly, $\operatorname{try} C_{k}$ also not involve performing the validation of the $T_{k}$ 's read set; the implementation simply sets status $[k]=$ committed and returns $C_{k}$.

Theorem 5.13. Algorithm 5.2 describes a weak TM implementation $M \in \mathcal{O F}$ such that in any execution $E$ of $M$, for every transaction $T \in t x n s(E), T$ performs $O(1)$ steps during the execution of any $t$-operation in $E$.

Proof. The proofs of opacity, TM-liveness and TM-progress are almost identical to the analogous proofs for Algorithm 5.1 .

(Weak disjoint-access parallelism) Consider any execution $E$ of Algorithm 5.2 and let $T_{i}$ and $T_{j}$ be any two transactions that contend on a base object $b$ in $E$. We need to prove that there is a path between a tobject in $\operatorname{Dset}\left(T_{i}\right)$ and a t-object in $\operatorname{Dset}\left(T_{j}\right)$ in $\tilde{G}\left(T_{i}, T_{j}, E\right)$ or there exists $X \in \operatorname{Dset}\left(T_{i}\right) \cap \operatorname{Dset}\left(T_{j}\right)$. Recall that there exists an edge between t-objects $X$ and $Y$ in $G\left(T_{i}, T_{j}, E\right)$ only if there exists a transaction $T \in \operatorname{txns}(E)$ such that $\{X, Y\} \in \operatorname{Dset}(T)$. 
- Suppose that $T_{i}$ and $T_{j}$ contend on base object $\operatorname{tvar}[\mathrm{m}]$ belonging to t-object $X_{m}$ in $E$. By Algorithm 5.2, a transaction accesses $X_{m}$ only if $X_{m}$ is contained in $\operatorname{Dset}\left(T_{m}\right)$. Thus, both $T_{i}$ and $T_{j}$ must access $X_{m}$.

- Suppose that $T_{i}$ and $T_{j}$ contend on base object status[i] in $E$ (the case when $T_{i}$ and $T_{j}$ contend on status[j] is symmetric). $T_{j}$ accesses status $[i]$ while performing a t-read of some t-object $X$ in Lines 4 and 10 only if $T_{i}$ is the owner of $X$. Also, $T_{j}$ accesses status $[i]$ while performing a t-write to $X$ in Lines 39 and 45 only if $T_{i}$ is the owner of $X$. But if $T_{i}$ is the owner of $X$, then $X \in \operatorname{Dset}\left(T_{i}\right)$.

- Suppose that $T_{i}$ and $T_{j}$ contend on base object status $[\mathrm{m}]$ belonging to some transaction $T_{m}$ in $E$. Firstly, observe that $T_{i}$ or $T_{j}$ access status $[m]$ only if there exist t-objects $X$ and $Y$ in $\operatorname{Dset}\left(T_{i}\right)$ and $\operatorname{Dset}\left(T_{j}\right)$ respectively such that $\{X, Y\} \in \operatorname{Dset}\left(T_{m}\right)$. This is because $T_{i}$ and $T_{j}$ would both read status $/ \mathrm{m}$ ] in Lines 4 (during t-read) and 39 (during t-write) only if $T_{m}$ was the previous owner of $X$ and $Y$. Secondly, one of $T_{i}$ or $T_{j}$ applies a nontrivial primitive to status $[m]$ only if $T_{i}$ and $T_{j}$ read status $[m]=$ live in Lines 4 (during t-read) and 37 (during t-write). Thus, at least one of $T_{i}$ or $T_{j}$ is concurrent to $T_{m}$ in $E$. It follows that there exists a path between $X$ and $Y$ in $\tilde{G}\left(T_{i}, T_{j}, E\right)$.

(Complexity) Since no implementation of any of the t-operation contains any iteration statements like for and while loops), the proof follows.

\subsection{Why Transactional memory should not be obstruction-free}

\begin{tabular}{|c|c|c|}
\hline & Obstruction-free TMs & Progressive TM $L P$ \\
\hline strict DAP & No [58] & Yes \\
\hline invisible reads+weak DAP & No & Yes \\
\hline stall complexity of t-reads & $\Omega(n)$ & $O(1)$ \\
\hline RAW /AWAR complexity & $\Omega(n)$ & $O(1)$ \\
\hline read-write base objects, wait-free TM-liveness & No [62] & Yes
\end{tabular}

Figure 5.3: Complexity gap between blocking and non-blocking TMs

As a synchronization abstraction, TM came as an alternative to conventional lock-based synchronization, and it therefore appears natural that early TM implementations [51, 77, 98, 117, avoided using locks. Instead, early TM designs relied on non-blocking synchronization, where a prematurely halted transaction cannot prevent all other transactions from committing. Possibly the weakest progress condition elucidating non-blocking TM-progress is obstruction-freedom.

However, in 2005, Ennals [47] argued that obstruction-free TMs inherently yield poor performance, because they require transactions to forcefully abort each other. Ennals further described a lock-based TM implementation [46] satisfying progressiveness that he claimed to outperform DSTM [77, the most referenced obstruction-free TM implementation at the time. Inspired by [47, more recent lock-based progressive TMs, such as TL [39], TL2 [38] and NOrec [35], demonstrate better performance than obstruction-free TMs on most workloads.

There is a considerable amount of empirical evidence on the performance gap between non-blocking (obstruction-free) and blocking (progressive) TM implementations but no analytical result explains it. We present complexity lower and upper bounds that provide such an explanation.

To exhibit a complexity gap between blocking and non-blocking TMs, we go back to the the progressive opaque TM implementation LP (Algorithm 4.1) that beats the impossibility result and the lower bounds we established for obstruction-free TMs. Recall that our implementation $L P,(1)$ uses only read-write base objects and provides wait-free TM-liveness, (2) ensures strict DAP, (3) has invisible reads, (4) performs $O(1)$ non-overlapping RAWs/AWARs per transaction, and (5) incurs $O(1)$ memory stalls for read operations (Theorem 4.13). In contrast, from prior work and our lower bounds we know that (i) no OF TM that provides wait-free transactional operations can be implemented using only read-write base objects [62]; (ii) no OF TM can provide strict DAP [58; (iii) no weak DAP OF TM has invisible reads 
(Section 5.2) and (iv) no OF TM ensures a constant number of stalls incurred by a t-read operation (Section 5.3. Finally, (v) no RW DAP opaque OF TM has constant RAW/AWAR complexity (Section 5.4). In fact, (iv) and (v) exhibit a linear separation between blocking and non-blocking TMs w.r.t expensive synchronization and memory stall complexity, respectively.

Altogether, our results exhibit a considerable complexity gap between progressive and obstruction-free TMs, as summarized in Figure 5.3, that seems to justify the shift in TM practice (circa. 2005) from non-blocking to blocking TMs.

Overcoming our lower bounds for obstruction-free TMs individually is comparatively easy. Say, TL [39] combines strict DAP with invisible reads, but it is not read-write, and it does not provide constant RAW/AWAR and stall complexities.

Coming out with a single algorithm that beats all these lower bounds is quite nontrivial. Our algorithm $L P$ incurs the cost of incremental validation, i.e., checking that the current read set has not changed per every new read operation. This is, however, unavoidable for invisible read algorithms (cf. Theorem 4.2), and is, in fact, believed to yield better performance in practice than "visible" reads [35, 39, 46, and we show that it enables constant stall and RAW/AWAR complexity.

\subsection{Related work and Discussion}

In this section, we summarize the results presented in this chapter and identify some unresolved questions.

Lower bounds for non-blocking TMs. Complexity of obstruction-free TMs was first studied by Guerraoui and Kapalka [58, 62, who proved that they cannot provide strict DAP. However, as we show in Section 5.5 it is possible to realize weaker than strict DAP variants of obstruction-free opaque TMs. Bushkov et al. [30] improved on the impossibility result in 58, and showed that a variant of strict DAP cannot be combined with obstruction-free TM-progress, even if a weaker (than strictly serializability) TMcorrectness property is assumed. In the thesis, we do not consider relaxations of strict serializability.

Guerraoui and Kapalka [58, 62] also proved that a strict serializable TM that provides OF TM-progress and wait-free TM-liveness cannot be implemented using only read and write primitives. An interesting open question is whether we can implement strict serializable TMs in $\mathcal{O F}$ using only read and write primitives.

Observe that, since there are at most $n$ concurrent transactions, we cannot do better than $(n-1)$ stalls (cf. Definition 2.18). Thus, the lower bound of Theorem 5.3 is tight.

Moreover, we conjecture that the linear (in $n$ ) lower bound of Theorem 5.4 for RW DAP opaque obstruction-free TMs can be strengthened to be linear in the size of the transaction's read set. Then, Algorithm 5.1. which proves a linear upper bound in the size of the transaction's read set, would allow us to establish a linear tight bound (in the size of the transaction's read set) for RW DAP opaque obstruction-free TMs.

Blocking versus non-blocking TMs. As highlighted in [39, 47, obstruction-free TMs typically must forcefully abort pending conflicting transactions. This observation inspires the impossibility of invisible reads (Theorem 5.1). Typically, to detect the presence of a conflicting transaction and abort it, the reading transaction must employ a RAW or a read-modify-write primitive like compare-and-swap, motivating the linear lower bound on expensive synchronization (Theorem 5.4). Also, in obstruction-free TMs, a transaction may not wait for a concurrent inactive transaction to complete and, as a result, we may have an execution in which a transaction incurs a distinct stall due to a transaction run by each other process, hence the linear stall complexity (Theorem 5.3). Intuitively, since transactions in progressive TMs may abort themselves in case of conflicts, they can employ invisible reads and maintain constant stall and RAW/AWAR complexities.

The lower bound and the proof technique in Theorem 5.3 is inspired by an analogous lower bound on linearizable solo-terminating implementations [15, 45] of a wide class of "perturbable" objects that 
include counters, compare-and-swap and single-writer snapshots [15, 45]. Informally, the definition of solo-termination (adapted to the TM context) says that for every finite execution $E$, and every transaction $T$ that is t-incomplete in $E$, there is a finite step contention-free extension in which $T$ eventually commits. Observe that, under this definition, $T$ is guaranteed to commit even in some executions that are not step contention-free for $T$. However, the definition of OF TM-progress used in the thesis ensures that $T$ is guaranteed to commit only if all its events are issued in the absence of step contention. Moreover, 15] described a single-lock (only the process holding the lock can invoke an operation) implementation of these objects that incurs $O(\log n)$ stalls, thus establishing a separation between the worst-case operation stall complexity of non-blocking and blocking (i.e., lock-based) implementations of these objects. In this chapter, we presented a linear separation in memory stall complexity between obstruction-free TMs and lock-based TMs characterized by progressiveness, which is a strictly stronger (than single-lock) progress guarantee, thus establishing the inherent cost of non-blocking progress in the TM context.

Some benefits of obstruction-free TMs, namely their ability to make progress even if some transactions prematurely fail, are not provided by progressive TMs. However, several papers [38, 39, 47, argued that lock-based TMs tend to outperform obstruction-free ones by allowing for simpler algorithms with lower overhead, and their inherent progress issues may be resolved using timeouts and contentionmanagers [112. This chapter explains the empirically observed performance gap between blocking and non-blocking TMs via a series of lower bounds on obstruction-free TMs and a progressive TM algorithm that beats all of them. 



\section{6}

\section{Lower bounds for partially non-blocking TMs}

\subsection{Overview}

It is easy to see that dynamic TMs where the patterns in which transactions access t-objects are not known in advance do not allow for wait-free TMs [62, i.e., every transaction must commit in a finite number of steps of the process executing it, regardless of the behavior of concurrent processes. Suppose that a transaction $T_{1}$ reads t-object $X$, then a concurrent transaction $T_{2}$ reads t-object $Y$, writes to $X$ and commits, and finally $T_{2}$ writes to $Y$. Since $T_{1}$ has read the "old" value in $X$ and $T_{2}$ has read the "old" value in $Y$, there is no way to commit $T_{1}$ and order the two transactions in a sequential execution. As this scenario can be repeated arbitrarily often, even the weaker guarantee of local progress that only requires that each transaction eventually commits if repeated sufficiently often, cannot be ensured by any strictly serializable TM implementation, regardless of the base objects it uses [31] ${ }^{1}$

But can we ensure that at least some transactions commit wait-free and what are the inherent costs? It is often argued that many realistic workloads are read-dominated: the proportion of read-only transactions is higher than that of updating ones, or read-only transactions have much larger data sets than updating ones [24, 63]. Therefore, it seems natural to require that read-only transactions commit wait-free. Since we are interested in complexity lower bounds, we require that updating transaction provide only sequential TM-progress.

First, we focus on strictly serializable TMs with the above TM-progress conditions that use invisible reads. We show that this requirement results in maintaining unbounded sets of versions for every data item, i.e., such implementations may not be practical due to their space complexity. Secondly, we prove that strictly serializable TMs with these progress conditions cannot ensure strict DAP. Thus, two transactions that access mutually disjoint data sets may prevent each other from committing. Thirdly, for weak DAP TMs, we show that a read-only transaction (with an arbitrarily large read set) must sometimes perform at least one expensive synchronization pattern [16] per t-read operation, i.e., the expensive synchronization complexity of a read-only transaction is linear in the size of its data set.

Formally, we denote by $\mathcal{R} \mathcal{W F}$ the class of partially non-blocking TMs.

Definition 6.1. (The class $\mathcal{R} \mathcal{W} \mathcal{F}$ ) $A T M$ implementation $M \in \mathcal{R} \mathcal{W} \mathcal{F}$ iff in its every execution:

\footnotetext{
${ }^{1}$ Note that the counter-example would not work if we imagine that the data sets accessed by a transaction can be known in advance. However, in the thesis, we consider the conventional dynamic TM programming model.
} 


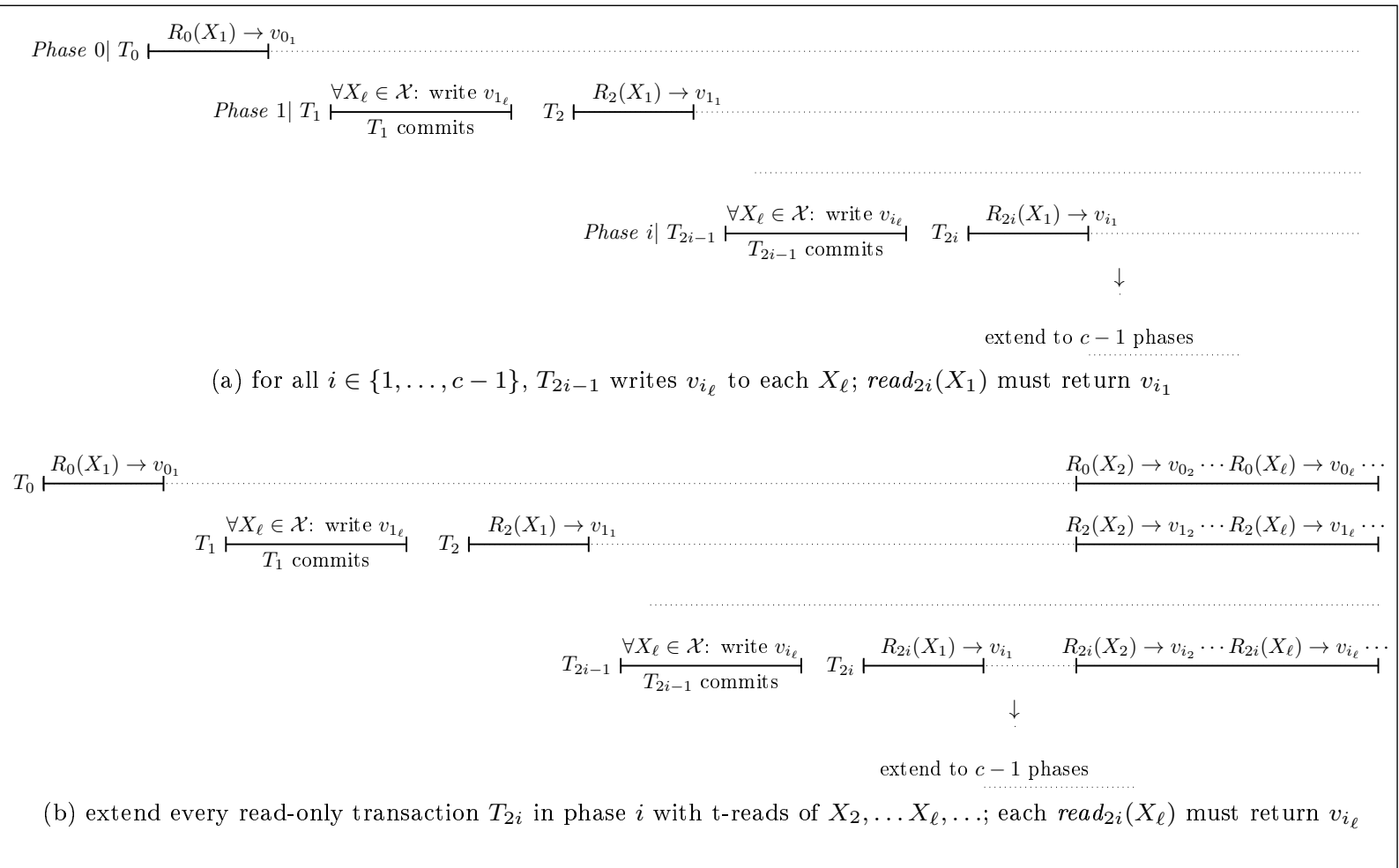

Figure 6.1: Executions in the proof of Theorem 6.1 execution in 6.1a must maintain $c$ distinct values of every t-object

- (wait-free TM-progress for read-only transactions) every read-only transaction commits in a finite number of its steps, and

- (sequential TM-progress and sequential TM-liveness for updating transactions) i.e., every transaction running step contention-free from a t-quiescent configuration, commits in a finite number of its steps.

Roadmap of Chapter 6. Section 6.2 presents a lower bound on the inherent space complexity of TMs in $\mathcal{R} \mathcal{W} \mathcal{F}$. Section 6.3 proves the impossibility of strict DAP TMs in $\mathcal{R} \mathcal{W} \mathcal{F}$ while in Section 6.4 assuming weak DAP, we prove a linear, in the size of the transaction's read set, lower bound on expensive synchronization complexity. We conclude this chapter with a discussion of the related work and open questions concerning TMs in $\mathcal{R W} \mathcal{F}$.

\subsection{The space complexity of invisible reads}

We prove that every strictly serializable TM implementation $M \in \mathcal{R} \mathcal{W} \mathcal{F}$ that uses invisible reads must keep unbounded sets of values for every t-object. To do so, for every $c \in \mathbb{N}$, we construct an execution of $M$ that maintains at least $c$ distinct values for every t-object. We require the following technical definition:

Definition 6.2. Let $E$ be any execution of a TM implementation $M$. We say that $E$ maintains $c$ distinct values $\left\{v_{1}, \ldots, v_{c}\right\}$ of t-object $X$, if there exists an execution $E \cdot E^{\prime}$ of $M$ such that

- $E^{\prime}$ contains the complete executions of $c$ t-reads of $X$ and,

- for all $i \in\{1, \ldots, c\}$, the response of the $i^{\text {th }} t$-read of $X$ in $E^{\prime}$ is $v_{i}$. 
Theorem 6.1. Let $M$ be any strictly serializable TM implementation in $\mathcal{R W \mathcal { F }}$ that uses invisible reads, and $\mathcal{X}$, any set of $t$-objects. Then, for every $c \in \mathbb{N}$, there exists an execution $E$ of $M$ such that $E$ maintains at least $c$ distinct values of each $t$-object $X \in \mathcal{X}$.

Proof. Let $v_{0_{\ell}}$ be the initial value of t-object $X_{\ell} \in \mathcal{X}$. For every $c \in \mathbb{N}$, we iteratively construct an execution $E$ of $M$ of the form depicted in Figure 6.1a. The construction of $E$ proceeds in phases: there are at most $c-1$ phases. For all $i \in\{0, \ldots c-1\}$, we denote the execution after phase $i$ as $E_{i}$ which is defined as follows:

- $E_{0}$ is the complete step contention-free execution fragment $\alpha_{0}$ of read-only transaction $T_{0}$ that performs $\operatorname{read}_{0}\left(X_{1}\right) \rightarrow v_{0_{1}}$

- for all $i \in\{1, \ldots, c-1\}, E_{i}$ is defined to be an execution of the form $\alpha_{0} \cdot \rho_{1} \cdot \alpha_{1} \cdots \rho_{i} \cdot \alpha_{i}$ such that for all $j \in\{1, \ldots, i\}$,

- $\rho_{j}$ is the t-complete step contention-free execution fragment of an updating transaction $T_{2 j-1}$ that, for all $X_{\ell} \in \mathcal{X}$ writes the value $v_{j_{\ell}}$ and commits

- $\alpha_{j}$ is the complete step contention-free execution fragment of a read-only transaction $T_{2 j}$ that performs $\operatorname{read}_{2 j}\left(X_{1}\right) \rightarrow v_{j_{1}}$

Since read-only transactions are invisible, for all $i \in\{0, \ldots, c-1\}$, the execution fragment $\alpha_{i}$ does not contain any nontrivial events. Consequently, for all $i<j \leq c-1$, the configuration after $E_{i}$ is indistinguishable to transaction $T_{2 j-1}$ from a t-quiescent configuration and it must be committed in $\rho_{j}$ (by sequential progress for updating transactions). Observe that, for all $1 \leq j<i, T_{2 j-1} \prec_{E}^{R T} T_{2 i-1}$. Strict serializability of $M$ now stipulates that, for all $i \in\{1, \ldots, c-1\}$, the t-read of $X_{1}$ performed by transaction $T_{2 i}$ in the execution fragment $\alpha_{i}$ must return the value $v_{i_{1}}$ of $X_{1}$ as written by transaction $T_{2 i-1}$ in the execution fragment $\rho_{i}$ (in any serialization, $T_{2 i-1}$ is the latest committed transaction writing to $X_{1}$ that precedes $T_{2 i}$ ). Thus, $M$ indeed has an execution $E$ of the form depicted in Figure 6.1a

Consider the execution fragment $E^{\prime}$ that extends $E$ in which, for all $i \in\{0, \ldots, c-1\}$, read-only transaction $T_{2 i}$ is extended with the complete execution of the t-reads of every t-object $X_{\ell} \in \mathcal{X} \backslash\left\{X_{1}\right\}$ (depicted in Figure 6.1b).

We claim that, for all $i \in\{0, \ldots, c-1\}$, and for all $X_{\ell} \in \mathcal{X} \backslash\left\{X_{1}\right\}$, $\operatorname{read}_{2 i}\left(X_{\ell}\right)$ performed by transaction $T_{2 i}$ must return the value $v_{i_{\ell}}$ of $X_{\ell}$ written by transaction $T_{2 i-1}$ in the execution fragment $\rho_{i}$. Indeed, by wait-free progress, $\operatorname{read}_{i}\left(X_{\ell}\right)$ must return a non-abort response in such an extension of $E$. Suppose by contradiction that $\operatorname{read}_{i}\left(X_{\ell}\right)$ returns a response that is not $v_{i_{\ell}}$. There are two cases:

- read $_{2 i}\left(X_{\ell}\right)$ returns the value $v_{j_{\ell}}$ written by transaction $T_{2 j-1} ; j<i$. However, since for all $j<i$, $T_{2 j} \prec_{E}^{R T} T_{2 i}$, the execution is not strictly serializable - contradiction.

- $\operatorname{read}_{2 i}\left(X_{\ell}\right)$ returns the value $v_{j_{\ell}}$ written by transaction $T_{2 j} ; j>i$. Since $\operatorname{read}_{i}\left(X_{1}\right)$ returns the value $v_{i_{1}}$ and $T_{2 i} \prec_{E}^{R T} T_{2 j}$, there exists no such serialization-contradiction.

Thus, $E$ maintains at least $c$ distinct values of every t-object $X \in \mathcal{X}$.

\subsection{Impossibility of strict DAP}

In this section, we prove that it is impossible to derive strictly serializable TM implementations in $\mathcal{R} \mathcal{W F}$ which ensure that any two transactions accessing pairwise disjoint data sets can execute without contending on the same base object.

Theorem 6.2. There exists no strictly serializable strict DAP TM implementation in $\mathcal{R} \mathcal{W F}$. 


(a) By strict DAP, $T_{0}$ and $T_{3}$ do not contend on any base object

Figure 6.2: Executions in the proof of Theorem 6.2 execution in $6.2 \mathrm{c}$ is not strictly serializable

Proof. Suppose by contradiction that there exists a strict DAP TM implementation $M \in \mathcal{R} \mathcal{W} \mathcal{F}$.

Let $v$ be the initial value of t-objects $X_{1}, X_{2}$ and $X_{3}$. Let $\pi$ be the t-complete step contention-free execution of transaction $T_{1}$ that writes the value $n v \neq v$ to t-objects $X_{1}$ and $X_{3}$. By sequential progress for updating transactions, $T_{1}$ must be committed in $\pi$.

Note that any read-only transaction that runs step contention-free after some prefix of $\pi$ must return a non-abort value. Since any such transaction reading $X_{1}$ or $X_{3}$ must return $v$ after the empty prefix of $\pi$ and $n v$ when it starts from $\pi$, there exists $\pi^{\prime}$, the longest prefix of $\pi$ that cannot be extended with the t-complete step contention-free execution of any transaction that performs a t-read of $X_{1}$ and returns $n v$ nor with the t-complete step contention-free execution of any transaction that performs a t-read of $X_{3}$ and returns $n v$.

Consider the execution fragment $\pi^{\prime} \cdot \alpha_{1}$, where $\alpha_{1}$ is the complete step contention-free execution of transaction $T_{0}$ that performs read $_{0}\left(X_{1}\right) \rightarrow v$. Indeed, by definition of $\pi^{\prime}$ and wait-free progress (assumed for read-only transactions), $M$ has an execution of the form $\pi^{\prime} \cdot \alpha_{1}$.

Let $e$ be the enabled event of transaction $T_{1}$ in the configuration after $\pi^{\prime}$. Without loss of generality, assume that $\pi^{\prime} \cdot e$ can be extended with the t-complete step contention-free execution of a transaction that reads $X_{3}$ and returns $n v$.

We now prove that $M$ has an execution of the form $\pi^{\prime} \cdot \alpha_{1} \cdot e \cdot \beta \cdot \gamma$, where

- $\beta$ is the t-complete step contention-free execution fragment of transaction $T_{3}$ that performs $\operatorname{read}_{3}\left(X_{3}\right) \rightarrow$ $n v$ and commits

- $\gamma$ is the t-complete step contention-free execution fragment of transaction $T_{2}$ that writes $n v$ to $X_{2}$ and commits.

Observe that, by definition of $\pi^{\prime}, M$ has an execution of the form $\pi^{\prime} \cdot e \cdot \beta$. By construction, transaction $T_{1}$ applies a nontrivial primitive to a base object, say $b$ in the event $e$ that is accessed by transaction $T_{3}$ in the execution fragment $\beta$. Since transactions $T_{0}$ and $T_{3}$ access mutually disjoint data sets in $\pi^{\prime} \cdot \alpha_{1} \cdot e \cdot \beta$, $T_{3}$ does not access any base object in $\beta$ to which transaction $T_{0}$ applies a nontrivial primtive in the 
execution fragment $\alpha_{1}$ (assumption of strict DAP). Thus, $\alpha_{1}$ does not contain a nontrivial primitive to $b$ and $\pi^{\prime} \cdot \alpha_{1} \cdot e \cdot \beta$ is indistinguishable to $T_{3}$ from the execution $\pi^{\prime} \cdot e \cdot \beta$. This proves that $M$ has an execution of the form $\pi^{\prime} \cdot \alpha_{1} \cdot e \cdot \beta$ (depicted in Figure 6.2a).

Since transaction $T_{2}$ writes to t-object $\operatorname{Dset}\left(T_{2}\right)=X_{2} \notin\left\{\operatorname{Dset}\left(T_{1}\right) \cup \operatorname{Dset}\left(T_{0}\right) \cup \operatorname{Dset}\left(T_{3}\right)\right\}$, by strict DAP, the configuration after $\pi^{\prime} \cdot \alpha_{1} \cdot e \cdot \beta$ is indistinguishable to $T_{2}$ from a t-quiescent configuration. Indeed, transaction $T_{2}$ does not contend with any of the transactions $T_{1}, T_{0}$ and $T_{3}$ on any base object in $\pi^{\prime} \cdot \alpha_{1} \cdot e \cdot \beta \cdot \gamma$. Sequential progress of $M$ requires that $T_{2}$ must be committed in $\pi^{\prime} \cdot \alpha_{1} \cdot e \cdot \beta \cdot \gamma$. Thus, $M$ has an execution of the form $\pi^{\prime} \cdot \alpha_{1} \cdot e \cdot \beta \cdot \gamma$.

By the above arguments, the execution $\pi^{\prime} \cdot \alpha_{1} \cdot e \cdot \beta \cdot \gamma$ is indistinguishable to each of the transactions $T_{1}, T_{0}, T_{2}$ and $T_{3}$ from $\gamma \cdot \pi^{\prime} \cdot \alpha_{1} \cdot e \cdot \beta$ in which transaction $T_{2}$ precedes $T_{1}$ in real-time ordering. Thus, $\gamma \cdot \pi^{\prime} \cdot \alpha_{1} \cdot e \cdot \beta$ is also an execution of $M$.

Consider the extension of the execution $\gamma \cdot \pi^{\prime} \cdot \alpha_{1} \cdot e \cdot \beta$ in which transaction $T_{0}$ performs $\operatorname{read}_{0}\left(X_{2}\right)$ and commits (depicted in Figure 6.2b). Strict serializability of $M$ stipulates that read $d_{0}\left(X_{2}\right)$ must return $n v$ since $T_{2}$ (which writes $n v$ to $X_{2}$ in $\gamma$ ) precedes $T_{0}$ in this execution.

Similarly, we now extend the execution $\pi^{\prime} \cdot \alpha_{1} \cdot e \cdot \beta \cdot \gamma$ with the complete step contention-free execution fragment of the t-read of $X_{2}$ by transaction $T_{0}$. Since $T_{0}$ is a read-only transaction, it must be committed in this extension. However, as proved above, this execution is indistinguishable to $T_{0}$ from the execution depicted in Figure $6.2 \mathrm{~b}$ in which read ${ }_{0}\left(X_{2}\right)$ must return $n v$. Thus, $M$ has an execution of the form $\pi^{\prime} \cdot \alpha_{1} \cdot e \cdot \beta \cdot \gamma \cdot \alpha_{2}$, where $T_{0}$ performs read $_{0}\left(X_{2}\right) \rightarrow n v$ in $\alpha_{2}$ and commits.

However, the execution $\pi^{\prime} \cdot \alpha_{1} \cdot e \cdot \beta \cdot \gamma \cdot \alpha_{2}$ (depicted in Figure 6.2c) is not strictly serializable. Transaction $T_{1}$ must be committed in any serialization and must precede transaction $T_{3}$ since $\operatorname{read}_{3}\left(X_{3}\right)$ returns the value of $X_{3}$ written by $T_{m}$. However, transaction $T_{0}$ must must precede $T_{1}$ since $\operatorname{read}_{0}\left(X_{1}\right)$ returns the initial the value of $X_{1}$. Also, transaction $T_{2}$ must precede $T_{0}$ since $\operatorname{read}_{0}\left(X_{2}\right)$ returns the value of $X_{2}$ written by $T_{2}$. But transaction $T_{3}$ must precede $T_{2}$ to respect real-time ordering of transactions. Thus, $T_{1}$ must precede $T_{0}$ in any serialization. But there exists no such serialization: a contradiction to the assumption that $M$ is strictly serializable.

\subsection{A linear lower bound on expensive synchronization for weak DAP}

In this section, we prove a linear lower bound (in the size of the transaction's read set) on the number of RAWs or AWARs for weak DAP TM implementations in $\mathcal{R} \mathcal{W} \mathcal{F}$. To do so, we construct an execution in which each t-read operation of an arbitrarily long read-only transaction contains a RAW or an AWAR.

Theorem 6.3. Every strictly serializable weakly DAP TM implementation $M \in \mathcal{R} \mathcal{W F}$ has, for all $m \in \mathbb{N}$, an execution in which some read-only transaction $T_{0}$ with $m=\mid$ Rset $\left(T_{0}\right) \mid$ performs $\Omega(m)$ RAWs/AWARs.

Proof. Let $v$ be the initial value of each of the t-objects $X_{1}, \ldots, X_{m}$. Consider the t-complete step contention-free execution of transaction $T_{0}$ that performs $m$ t-reads $\operatorname{read}_{0}\left(X_{1}\right), \operatorname{read}_{0}\left(X_{1}\right), \ldots \operatorname{read}_{0}\left(X_{m}\right)$ and commits. We prove that each of the first $m-1$ t-reads must perform a RAW or an AWAR.

For all $j \in\{1, \ldots, m-1\}, M$ has an execution of the form $\alpha_{1} \cdot \alpha_{2} \cdots \alpha_{j}$, where for all $i \in\{1, \ldots, j\}, \alpha_{i}$ is the complete step contention-free execution fragment of $\operatorname{read}_{0}\left(X_{j}\right) \rightarrow v$. Assume inductively that each of the first $j-1$ t-reads performs a RAW or an AWAR in this execution. We prove that $\operatorname{read}_{0}\left(X_{j}\right)$ must perform a RAW or an AWAR in the execution fragment $\alpha_{j}$. Suppose by contradiction that $\alpha_{j}$ does not contain a RAW or an AWAR.

The following claim shows that we can schedule a committed transaction $T_{j}$ that writes a new value to $X_{j}$ concurrent to $\operatorname{read}_{0}\left(X_{j}\right)$ such that the execution is indistinguishable to both $T_{0}$ and $T_{j}$ from a step contention-free execution (depicted in Figure 6.3a). 
Chapter 6 Lower bounds for patially non-blocking TMs

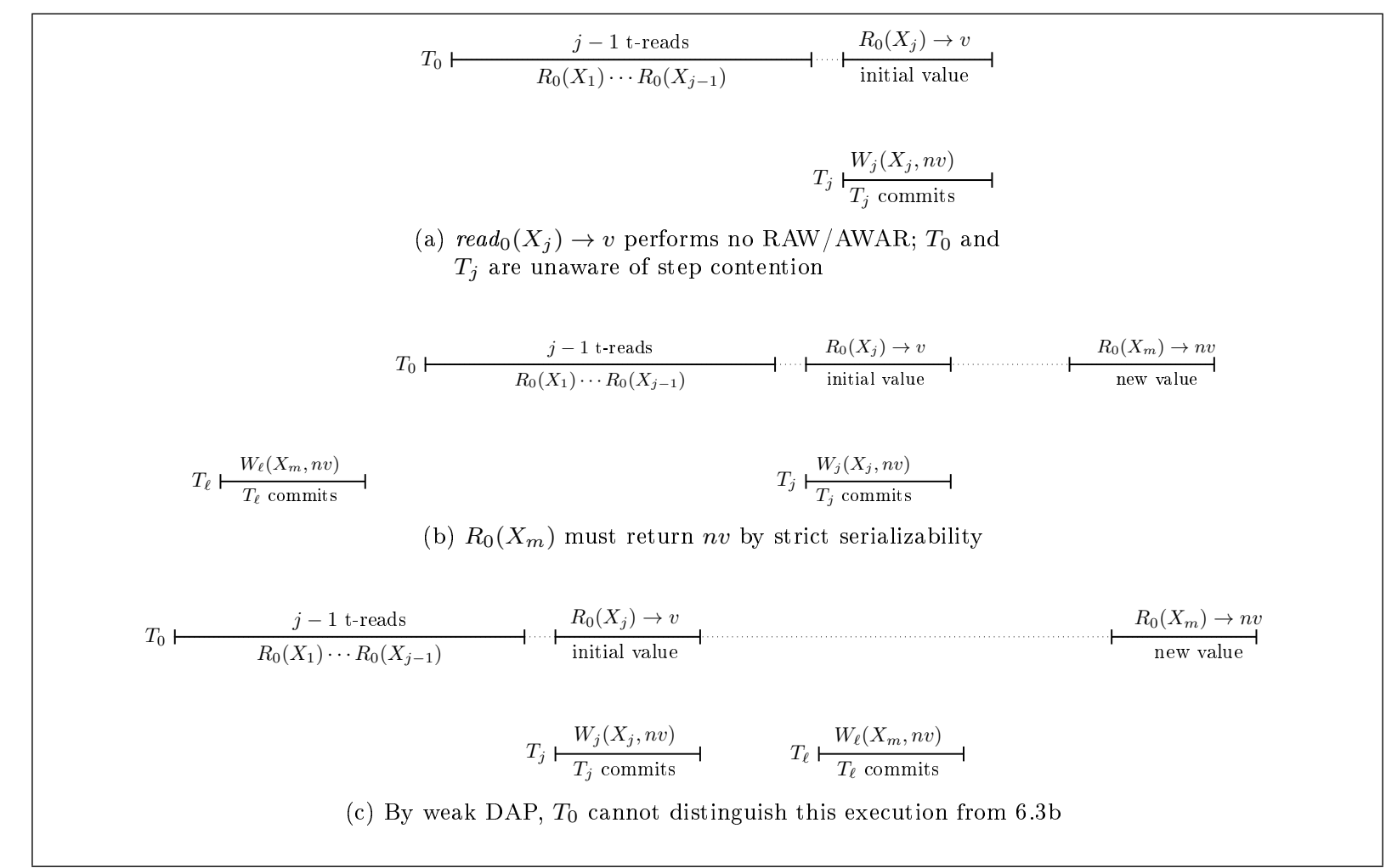

Figure 6.3: Executions in the proof of Theorem 6.3 execution in 6.3c is not strictly serializable

Claim 6.4. For all $j \in\{1, \ldots, m-1\}, M$ has an execution of the form $\alpha_{1} \cdots \alpha_{j-1} \cdot \alpha_{j}^{1} \cdot \delta_{j} \cdot \alpha_{j}^{2}$ where,

- $\delta_{j}$ is the $t$-complete step contention-free execution fragment of transaction $T_{j}$ that writes $n v \neq v$ and commits

- $\alpha_{j}^{1} \cdot \alpha_{j}^{2}=\alpha_{j}$ is the complete execution fragment of the $j^{\text {th }} t$-read read $d_{0}\left(X_{j}\right) \rightarrow v$ such that

$-\alpha_{j}^{1}$ does not contain any nontrivial events

$-\alpha_{1} \cdots \alpha_{j-1} \cdot \alpha_{j}^{1} \cdot \delta_{j} \cdot \alpha_{j}^{2}$ is indistinguishable to $T_{0}$ from the step contention-free execution fragment $\alpha_{1} \cdots \alpha_{j-1} \cdot \alpha_{j}^{1} \cdot \alpha_{j}^{2}$

Moreover, $T_{j}$ does not access any base object to which $T_{0}$ applies a nontrivial event in $\alpha_{1} \cdots \alpha_{j-1} \cdot \alpha_{j}^{1} \cdot \delta_{j}$.

Proof. By wait-free progress (for read-only transactions) and strict serializability, $M$ has an execution of the form $\alpha_{1} \cdots \alpha_{j-1}$ in which each of the t-reads performed by $T_{0}$ must return the initial value of the t-objects.

Since $T_{j}$ is an updating transaction, by sequential progress, there exists an execution of $M$ of the form $\delta_{j} \cdot \alpha_{1} \cdots \alpha_{j-1}$. Since $T_{0}$ and $T_{j}$ are disjoint-access in the $\delta_{j} \cdot \alpha_{1} \cdots \alpha_{j-1}$, by Lemma $2.10, T_{0}$ and $T_{j}$ do not contend on any base object in $\delta_{j} \cdot \alpha_{1} \cdots \alpha_{j-1}$. Thus, $\alpha_{1} \cdots \alpha_{j-1} \cdot \delta_{j}$ is indistinguishable to $T_{j}$ from the execution $\delta_{j}$ and $\alpha_{1} \cdots \alpha_{j-1} \cdot \delta_{j}$ is also an execution of $M$.

Let $e$ be the first event that contains a write to a base object in $\alpha_{j}$. If there exists no such write event to a base object in $\alpha_{j}$, then $\alpha_{j}^{1}=\alpha_{j}$ and $\alpha_{j}^{2}$ is empty. Otherwise, we represent the execution fragment $\alpha_{j}$ as $\alpha_{j}^{1} \cdot e \cdot \alpha_{j}^{f}$.

Since $\alpha_{j}^{s}$ does not contain any nontrivial events that write to a base object, $\alpha_{1} \cdots \alpha_{j-1} \cdot \alpha_{j}^{s} \cdot \delta_{j}$ is indistinguishable to transaction $T_{j}$ from the execution $\alpha_{1} \cdots \alpha_{j-1} \cdot \delta_{j}$. Thus, $\alpha_{1} \cdots \alpha_{j-1} \cdot \alpha_{j}^{s} \cdot \delta_{j}$ is an execution of $M$. Since $e$ is not an atomic-write-after-read, $\alpha_{1} \cdots \alpha_{j-1} \cdot \alpha_{j}^{s} \cdot \delta_{j} \cdot e$ is an execution of $M$. Since $\alpha_{j}$ does not contain a RAW, any read performed in $\alpha_{j}^{f}$ may only be performed to base objects 
previously written in $e \cdot \alpha_{j}^{f}$. Thus, $\alpha_{1} \cdots \alpha_{j-1} \cdot \alpha_{j}^{s} \cdot \delta_{j} \cdot e \cdot \alpha_{j}^{f}$ is indistinguishable to transaction $T_{0}$ from the step contention-free execution $\alpha_{1} \cdots \alpha_{j-1} \cdot \alpha_{j}^{s} \cdot e \cdot \alpha_{j}^{f}$ in which $\operatorname{read}_{0}\left(X_{j}\right) \rightarrow v$.

Choosing $\alpha_{j}^{2}=e \cdot \alpha_{j}^{f}$, it follows that $M$ has an execution of the form $\alpha_{1} \cdots \alpha_{j-1} \cdot \alpha_{j}^{1} \cdot \delta_{j} \cdot \alpha_{j}^{2}$ that is indistinguishable to $T_{j}$ and $T_{0}$ from a step contention-free execution. The proof follows.

We now prove that, for all $j \in\{1, \ldots, m-1\}, M$ has an execution of the form $\delta_{m} \cdot \alpha_{1} \cdots \alpha_{j-1} \cdot \alpha_{j}^{1} \cdot \delta_{j} \cdot \alpha_{j}^{2}$ such that

- $\delta_{m}$ is the t-complete step contention-free execution of transaction $T_{\ell}$ that writes $n v \neq v$ to $X_{m}$ and commits

- $T_{\ell}$ and $T_{0}$ do not contend on any base object in $\delta_{m} \cdot \alpha_{1} \cdots \alpha_{j-1} \cdot \alpha_{j}^{1} \cdot \delta_{j} \cdot \alpha_{j}^{2}$

- $T_{\ell}$ and $T_{j}$ do not contend on any base object in $\delta_{m} \cdot \alpha_{1} \cdots \alpha_{j-1} \cdot \alpha_{j}^{1} \cdot \delta_{j} \cdot \alpha_{j}^{2}$.

By sequential progress for updating transactions, $T_{\ell}$ which writes the value $n v$ to $X_{m}$ must be committed in $\delta_{m}$ since it is running in the absence of step-contention from the initial configuration. Observe that $T_{\ell}$ and $T_{0}$ are disjoint-access in $\delta_{m} \cdot \alpha_{1} \cdots \alpha_{j-1} \cdot \alpha_{j}^{1} \cdot \delta_{j} \cdot \alpha_{j}^{2}$. By definition of $\alpha_{j}^{1}$ and $\alpha_{j}^{2}, \delta_{m} \cdot \alpha_{1} \cdots \alpha_{j-1} \cdot \alpha_{j}^{1} \cdot \delta_{j} \cdot \alpha_{j}^{2}$ is indistinguishable to $T_{0}$ from $\delta_{m} \cdot \alpha_{1} \cdots \alpha_{j-1} \cdot \alpha_{j}^{1} \cdot \alpha_{j}^{2}$. By Lemma $2.10 T_{\ell}$ and $T_{0}$ do not contend on any base object in $\delta_{m} \cdot \alpha_{1} \cdots \alpha_{j-1} \cdot \alpha_{j}^{1} \cdot \alpha_{j}^{2}$.

By Claim 6.4 $\delta_{m} \cdot \alpha_{1} \cdots \alpha_{j-1} \cdot \alpha_{j}^{1} \cdot \delta_{j}$ is indistinguishable to $T_{j}$ from $\delta_{m} \cdot \delta_{j}$. But transactions $T_{\ell}$ and $T_{j}$ are disjoint-access in $\delta_{m} \cdot \delta_{j}$, and by Lemma 2.10, $T_{j}$ and $T_{\ell}$ do not contend on any base object in $\delta_{m} \cdot \delta_{j}$.

Since strict serializability of $M$ stipulates that each of the $j$ t-reads performed by $T_{0}$ return the initial values of the respective t-objects, $M$ has an execution of the form $\delta_{m} \cdot \alpha_{1} \cdots \alpha_{j-1} \cdot \alpha_{j}^{1} \cdot \delta_{j} \cdot \alpha_{j}^{2}$.

Consider the extension of $\delta_{m} \cdot \alpha_{1} \cdots \alpha_{j-1} \cdot \alpha_{j}^{1} \cdot \delta_{j} \cdot \alpha_{j}^{2}$ in which $T_{0}$ performs $(m-j)$ t-reads of $X_{j+1}, \cdots, X_{m}$ step contention-free and commits (depicted in Figure 6.3b). By wait-free progress of $M$ and since $T_{0}$ is a read-only transaction, there exists such an execution. Notice that the $m^{\text {th }}$ t-read, $\operatorname{read}_{0}\left(X_{m}\right)$ must return the value $n v$ by strict serializability since $T_{\ell}$ precedes $T_{0}$ in real-time order in this execution.

Recall that neither pairs of transactions $T_{\ell}$ and $T_{j}$ nor $T_{\ell}$ and $T_{0}$ contend on any base object in the execution $\delta_{m} \cdot \alpha_{1} \cdots \alpha_{j-1} \cdot \alpha_{j}^{1} \cdot \delta_{j} \cdot \alpha_{j}^{2}$. It follows that for all $j \in\{1, \ldots, m-1\}, M$ has an execution of the form $\alpha_{1} \cdots \alpha_{j-1} \cdot \alpha_{j}^{1} \cdot \delta_{j} \cdot \alpha_{j}^{2} \cdot \delta_{m}$ in which $T_{j}$ precedes $T_{\ell}$ in real-time order.

Let $\alpha^{\prime}$ be the execution fragment that extends $\alpha_{1} \cdots \alpha_{j-1} \cdot \alpha_{j}^{1} \cdot \delta_{j} \cdot \alpha_{j}^{2} \cdot \delta_{m}$ in which $T_{0}$ performs $(m-j)$ t-reads of $X_{j+1}, \cdots, X_{m}$ step contention-free and commits (depicted in Figure 6.3c). Since $\alpha_{1} \cdots \alpha_{j-1}$. $\alpha_{j}^{1} \cdot \delta_{j} \cdot \alpha_{j}^{2} \cdot \delta_{m}$ is indistinguishable to $T_{0}$ from the execution $\delta_{m} \cdot \alpha_{1} \cdots \alpha !_{j-1} \cdot \alpha_{j}^{1} \cdot \delta_{j} \cdot \alpha_{j}^{2}, \operatorname{read}_{0}\left(X_{m}\right)$ must return the response value $n v$ in $\alpha^{\prime}$.

The execution $\alpha_{1} \cdots \alpha_{j-1} \cdot \alpha_{j}^{1} \cdot \delta_{j} \cdot \alpha_{j}^{2} \cdot \delta_{m} \cdot \alpha^{\prime}$ is not strictly serializable. In any serialization, $T_{j}$ must precede $T_{\ell}$ to respect the real-time ordering of transactions, while $T_{\ell}$ must precede $T_{0}$ since $\operatorname{read}_{j}\left(X_{m}\right)$ returns the value of $X_{m}$ updated by $T_{\ell}$. Also, transaction $T_{0}$ must precede $T_{j}$ since $\operatorname{read}_{0}\left(X_{j}\right)$ returns the initial value of $X_{j}$. But there exists no such serialization: a contradiction to the assumption that $M$ is strict serializable.

Thus, for all $j \in\{1, \ldots, m-1\}$, transaction $T_{0}$ must perform a RAW or an AWAR during the execution of $\operatorname{read}_{0}\left(X_{j}\right)$, completing the proof.

Since Theorem 6.3 implies that read-only transactions must perform nontrivial events, we have the following corollary that was proved directly in [23].

Corollary 6.5 ([23]). There does not exist any strictly serializable weak DAP TM implementation $M \in \mathcal{R} \mathcal{W F}$ that uses invisible reads. 


\subsection{Related work and Discussion}

Attiya et al. 223. showed that it is impossible to implement weak DAP strictly serializable TMs in $\mathcal{R W F}$ if read-only transactions may only apply trivial primitives to base objects. Attiya et al. [23] also considered a stronger "disjoint-access" property, called simply DAP, referring to the original definition proposed Israeli and Rappoport [84. In DAP, two transactions are allowed to concurrently access (even for reading) the same base object only if they are disjoint-access. For an $n$-process DAP TM implementation, it is shown in 23 that a read-only transaction must perform at least $n-3$ writes. Our lower bound in Theorem 6.3 is strictly stronger than the one in [23, as it assumes only weak DAP, considers a more precise RAW/AWAR metric, and does not depend on the number of processes in the system. (Technically, the last point follows from the fact that the execution constructed in the proof of Theorem 6.3 uses only 3 concurrent processes.) Thus, the theorem subsumes the two lower bounds of [23] within a single proof.

Perelman et al. [107] considered the closely related (to $\mathcal{R W F}$ ) class of mv-permissive TMs: a transaction can only be aborted if it is an updating transaction that conflicts with another updating transaction. $\mathcal{R W F}$ is incomparable with the class of mv-permissive TMs. On the one hand, mv-permissiveness guarantees that read-only transactions never abort, but does not imply that they commit in a waitfree manner. On the other hand, $\mathcal{R W F}$ allows an updating transaction to abort in the presence of a concurrent read-only transaction, which is disallowed by mv-permissive TMs. Observe that, technically, mv-permissiveness is a blocking TM-progress condition, although when used in conjunction with wait-free TM-liveness, it is a partially non-blocking TM-progress condition that is strictly stronger than $\mathcal{R} \mathcal{W} \mathcal{F}$.

Assuming starvation-free TM-liveness, [107] showed that implementing a weak DAP strictly serializable mv-permissive TM is impossible. In the thesis, we showed that strictly serializable TMs in $\mathcal{R} \mathcal{W F}$ cannot provide strict DAP, but proving the impossibility result assuming weak DAP remains an interesting open question.

[107. also proved that mv-permissive TMs cannot be online space optimal, i.e., no mv-permissive TM can keep the minimum number of old object versions for any TM history. Our result on the space complexity of implementations in $\mathcal{R} \mathcal{W F}$ that use invisible reads (Theorem 6.1) is different since it proves that the implementation must maintain an unbounded number of versions of every t-object. Our proof technique can however be used to show that mv-permissive TMs considered in [107. should also maintain unbounded number of versions. 


\section{Hybrid transactional memory (HyTM)}

HAL: The 9000 series is the most reliable computer ever made. No 9000 computer has ever made a mistake or distorted information. We are all, by any practical definition of the words, foolproof and incapable of error.

$\cdots$

HAL: I've just picked up a fault in the AE35 unit. It's going to go $100 \%$ failure in 72 hours.

HAL: It can only be attributable to human error.

Stanley Kubrick-2001: A Space Odyssey

\subsection{Overview}

Hybrid transactional memory. The TM abstraction, in its original manifestation from the proposal by Herlihy and Moss [78, augmented the processor's cache-coherence protocol and extended the CPU's instruction set with instructions to indicate which memory accesses must be transactional [78. Most popular TM designs, subsequent to the original proposal in [78. have implemented all the functionality in software [35, 51, 77, 98, 114] (cf. software TM model in Chapter 2). More recently, CPUs have included hardware extensions to support short, small hardware transactions [1, 104, 108.

Early experience with programming Hardware transactional memory (HTM), e.g. [7, 37, 43, paints an interesting picture: if used carefully, HTM can be an extremely useful construct, and can significantly speed up and simplify concurrent implementations. At the same time, this powerful tool is not without its limitations: since HTMs are usually implemented on top of the cache coherence mechanism, hardware transactions have inherent capacity constraints on the number of distinct memory locations that can be accessed inside a single transaction. Moreover, all current proposals are best-effort, as they may abort 
under imprecisely specified conditions (cache capacity overflow, interrupts etc). In brief, the programmer should not solely rely on HTMs.

Several Hybrid Transactional Memory (HyTM) schemes [34, 36, 86, 96, have been proposed to complement the fast, but best-effort nature of HTM with a slow, reliable software transactional memory (STM) backup. These proposals have explored a wide range of trade-offs between the overhead on hardware transactions, concurrent execution of hardware and software, and the provided progress guarantees.

Early proposals for HyTM implementations [36, 86] shared some interesting features. First, transactions that do not conflict are expected to run concurrently, regardless of their types (software or hardware). This property is referred to as progressiveness [61] and is believed to allow for increased parallelism. Second, in addition to changing the values of transactional objects, hardware transactions usually employ code instrumentation techniques. Intuitively, instrumentation is used by hardware transactions to detect concurrency scenarios and abort in the case of contention. The number of instrumentation steps performed by these implementations within a hardware transaction is usually proportional to the size of the transaction's data set.

Recent work by Riegel et al. [110] surveyed the various HyTM algorithms to date, focusing on techniques to reduce instrumentation overheads in the frequently executed hardware fast-path. However, it is not clear whether there are fundamental limitations when building a HyTM with non-trivial concurrency between hardware and software transactions. In particular, what are the inherent instrumentation costs of building a HyTM, and what are the trade-offs between these costs and the provided concurrency, i.e., the ability of the HyTM system to run software and hardware transactions in parallel?

Modelling HyTM. To address these questions, the thesis proposes the first model for hybrid TM systems which formally captures the notion of cached accesses provided by hardware transactions, and precisely defines instrumentation costs in a quantifiable way.

We model a hardware transaction as a series of memory accesses that operate on locally cached copies of the variables, followed by a cache-commit operation. In case a concurrent transaction performs a (read-write or write-write) conflicting access to a cached object, the cached copy is invalidated and the hardware transaction aborts.

Our model for instrumentation is motivated by recent experimental evidence which suggests that the overhead on hardware transactions imposed by code which detects concurrent software transactions is a significant performance bottleneck [99. In particular, we say that a HyTM implementation imposes a logical partitioning of shared memory into data and metadata locations. Intuitively, metadata is used by transactions to exchange information about contention and conflicts while data locations only store the values of data items read and updated within transactions. We quantify instrumentation cost by measuring the number of accesses to metadata objects which transactions perform. Our framework captures all known HyTM proposals which combine HTMs with an STM fallback [34, 36, 86, 96, 109].

The cost of instrumentation. Once this general model is in place, we derive two lower bounds on the cost of implementing a HyTM. First, we show that some instrumentation is necessary in a HyTM implementation even if we only intend to provide sequential progress, where a transaction is only guaranteed to commit if it runs in the absence of concurrency.

Second, we prove that any progressive HyTM implementation providing obstruction-free liveness (every operation running solo returns some response) and has executions in which an arbitrarily long readonly hardware transaction running in the absence of concurrency must access a number of distinct metadata objects proportional to the size of its data set. Our proof technique is interesting in its own right. Inductively, we start with a sequential execution in which a "large" set $S_{m}$ of read-only hardware transactions, each accessing $m$ distinct data items and $m$ distinct metadata memory locations, run after an execution $E_{m}$. We then construct execution $E_{m+1}$, an extension of $E_{m}$, which forces at least half of the transactions in $S_{m}$ to access a new metadata base object when reading a new $(m+1)^{t h}$ data item, running after $E_{m+1}$. The technical challenge, and the key departure from prior work on STM lower bounds, e.g. [23, 58, 62], is that hardware transactions practically possess "automatic" conflict detection, aborting on contention. This is in contrast to STMs, which must take steps to detect contention on memory locations. 
We match this lower bound with an HyTM algorithm that, additionally, allows for uninstrumented writes and invisible reads and is provably opaque [62. To the best of our knowledge, this is the first formal proof of correctness of a HyTM algorithm.

Low-instrumentation HyTM. The high instrumentation costs of early HyTM designs, which we show to be inherent, stimulated more recent HyTM schemes [34, 96, 99, 110, to sacrifice progressiveness for constant instrumentation cost (i.e., not depending on the size of the transaction). In the past two years, Dalessandro et al. [34] and Riegel et al. [110] have proposed HyTMs based on the efficient NOrec STM [35. These HyTMs schemes do not guarantee any parallelism among transactions; only sequential progress is ensured. Despite this, they are among the best-performing HyTMs to date due to the limited instrumentation in hardware transactions.

Starting from this observation, we provide a more precise upper bound for low-instrumentation HyTMs by presenting a HyTM algorithm with invisible reads and uninstrumented hardware writes which guarantees that a hardware transaction accesses at most one metadata object in the course of its execution. Software transactions in this implementation remain progressive, while hardware transactions are guaranteed to commit only if they do not run concurrently with an updating software transaction (or exceed capacity). Therefore, the cost of avoiding the linear lower bound for progressive implementations is that hardware transactions may be aborted by non-conflicting software ones.

Roadmap of Chapter 7, In Section 7.2, we introduce the model of HyTMs and Section 7.3 studies the inherent cost of concurrency in progressive HyTMs by presenting a linear lower bound on the cost of instrumentation while Section 7.4 presents a matching upper bound. In Section 7.5 discusses providing partial concurrency with instrumentation cost and in Section 7.6. we elaborate on prior work related to HyTMs.

\subsection{Modelling HyTM}

In this chapter, we introduce the model of HyTMs, extending the TM model from Chapter 2 , that intuitively captures the cache-coherence protocols employed in shared memory systems.

\subsubsection{Direct and cached accesses}

We now describe the operation of a Hybrid Transactional Memory (HyTM) implementation. In our model, every base object can be accessed with two kinds of primitives, direct and cached.

In a direct access, the rmw primitive operates on the memory state: the direct-access event atomically reads the value of the object in the shared memory and, if necessary, modifies it.

In a cached access performed by a process $i$, the rmw primitive operates on the cached state recorded in process $i$ 's tracking set $\tau_{i}$. One can think of $\tau_{i}$ as the $L 1$ cache of process $i$. A hardware transaction is a series of cached rmw primitives performed on $\tau_{i}$ followed by a cache-commit primitive.

More precisely, $\tau_{i}$ is a set of triples $(b, v, m)$ where $b$ is a base object identifier, $v$ is a value, and $m \in$ $\{$ shared, exclusive $\}$ is an access mode. The triple $(b, v, m)$ is added to the tracking set when $i$ performs a cached rmw access of $b$, where $m$ is set to exclusive if the access is nontrivial, and to shared otherwise. We assume that there exists some constant TS (representing the size of the L1 cache) such that the condition $\left|\tau_{i}\right| \leq T S$ must always hold; this condition will be enforced by our model. A base object $b$ is present in $\tau_{i}$ with mode $m$ if $\exists v,(b, v, m) \in \tau_{i}$.

A trivial (resp. nontrivial) cached primitive $\langle g, h\rangle$ applied to $b$ by process $i$ first checks the condition $\left|\tau_{i}\right|=T S$ and if so, it sets $\tau_{i}=\emptyset$ and immediately returns $\perp$ (we call this event a capacity abort). We assume that $T S$ is large enough so that no transaction with data set of size 1 can incur a capacity abort. If the transaction does not incur a capacity abort, the process checks whether $b$ is present in exclusive (resp. any) mode in $\tau_{j}$ for any $j \neq i$. If so, $\tau_{i}$ is set to $\emptyset$ and the primitive returns $\perp$. Otherwise, the triple $(b, v$, shared $)$ (resp. $(b, g(v)$, exclusive $))$ is added to $\tau_{i}$, where $v$ is the most recent cached value of 


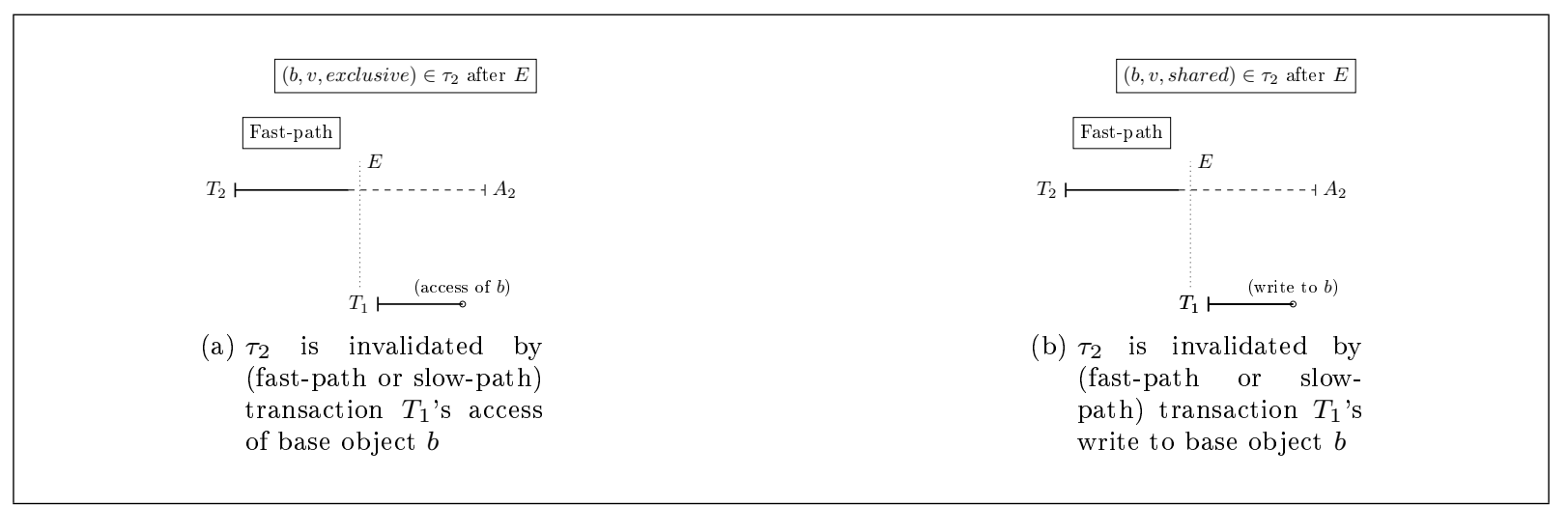

Figure 7.1: Tracking set aborts in fast-path transactions; we denote a fast-path (and resp. slow-path) transaction by $F$ (and resp. $S$ )

$b$ in $\tau_{i}$ (in case $b$ was previously accessed by $i$ within the current hardware transaction) or the value of $b$ in the current memory configuration, and finally $h(v)$ is returned.

A tracking set can be invalidated by a concurrent process: if, in a configuration $C$ where $(b, v$, exclusive $) \in$ $\tau_{i}$ (resp. $(b, v$, shared $) \in \tau_{i}$ ), a process $j \neq i$ applies any primitive (resp. any nontrivial primitive) to $b$, then $\tau_{i}$ becomes invalid and any subsequent cached primitive invoked by $i$ sets $\tau_{i}$ to $\emptyset$ and returns $\perp$. We refer to this event as a tracking set abort.

Finally, the cache-commit primitive issued by process $i$ with a valid $\tau_{i}$ does the following: for each base object $b$ such that $(b, v$, exclusive $) \in \tau_{i}$, the value of $b$ in $C$ is updated to $v$. Finally, $\tau_{i}$ is set to $\emptyset$ and the primitive returns commit.

Note that HTM may also abort spuriously, or because of unsupported operations [108. The first cause can be modelled probabilistically in the above framework, which would not however significantly affect our claims and proofs, except for a more cumbersome presentation. Also, our lower bounds are based exclusively on executions containing t-reads and t-writes. Therefore, in the following, we only consider contention and capacity aborts.

\subsubsection{Slow-path and fast-path transactions}

In the following, we partition HyTM transactions into fast-path transactions and slow-path transactions. Practically, two separate algorithms (fast-path one and slow-path one) are provided for each t-operation.

A slow-path transaction models a regular software transaction. An event of a slow-path transaction is either an invocation or response of a t-operation, or a rmw primitive on a base object.

A fast-path transaction essentially encapsulates a hardware transaction. An event of a fast-path transaction is either an invocation or response of a t-operation, a cached primitive on a base object, or a cache-commit: $t$-read and $t$-write are only allowed to contain cached primitives, and $\operatorname{try} C$ consists of invoking cache-commit. Furthermore, we assume that a fast-path transaction $T_{k}$ returns $A_{k}$ as soon an underlying cached primitive or cache-commit returns $\perp$. Figure 7.1 depicts such a scenario illustrating a tracking set abort: fast-path transaction $T_{2}$ executed by process $p_{2}$ accesses a base object $b$ in shared (and resp. exclusive) mode and it is added to its tracking set $\tau_{2}$. Immediately after the access of $b$ by $T_{2}$, a concurrent transaction $T_{1}$ applies a nontrivial primitive to $b$ (and resp. accesses $b$ ). Thus, the tracking of $p_{2}$ is invalidated and $T_{2}$ must be aborted in any extension of this execution.

We provide two key observations on this model regarding the interactions of non-committed fast path transactions with other transactions. Let $E$ be any execution of a HyTM implementation $\mathcal{M}$ in which a fast-path transaction $T_{k}$ is either t-incomplete or aborted. Then the sequence of events $E^{\prime}$ derived by removing all events of $E \mid k$ from $E$ is an execution $\mathcal{M}$. Moreover: 


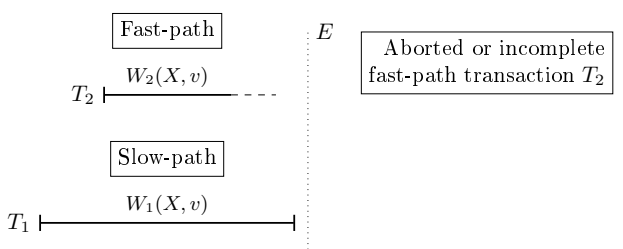

(a)

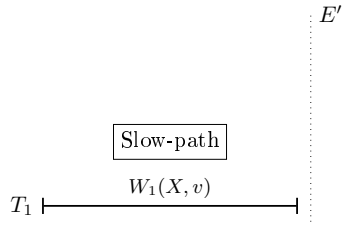

(b)

Figure 7.2: Execution $E$ in Figure $7.2 \mathrm{a}$ is indistinguishable to $T_{1}$ from the execution $E^{\prime}$ in Figure $7.2 \mathrm{~b}$

Observation 7.1. To every slow-path transaction $T_{m} \in \operatorname{txns}(E), E$ is indistinguishable from $E^{\prime}$.

Observation 7.2. If a fast-path transaction $T_{m} \in \operatorname{txns}(E) \backslash\left\{T_{k}\right\}$ does not incur a tracking set abort in $E$, then $E$ is indistinguishable to $T_{m}$ from $E^{\prime}$.

Intuitively, these observations say that fast-path transactions which are not yet committed are invisible to slow-path transactions, and can communicate with other fast-path transactions only by incurring their tracking-set aborts. Figure 7.2 illustrates Observation 7.1 a fast-path transaction $T_{2}$ is concurrent to a slow-path transaction $T_{1}$ in an execution $E$. Since $T_{2}$ is t-incomplete or aborted in this execution, $E$ is indistinguishable to $T_{1}$ from an execution $E^{\prime}$ derived by removing all events of $T_{2}$ from $E$. Analogously, to illustrate Observation 7.2 , if $T_{1}$ is a fast-path transaction that does not incur a tracking set abort in $E$, then $E$ is indistinguishable to $T_{1}$ from $E^{\prime}$.

\subsubsection{Instrumentation}

Now we define the notion of code instrumentation in fast-path transactions. Intuitively, instrumentation characterizes the number of extra "metadata" accesses performed by a fast-path transaction.

We start with the following technical definition. An execution $E$ of a HyTM $\mathcal{M}$ appears t-sequential to a transaction $T_{k} \in \operatorname{txns}(E)$ if there exists an execution $E^{\prime}$ of $\mathcal{M}$ such that:

- $\operatorname{txns}\left(E^{\prime}\right) \subseteq \operatorname{txns}(E) \backslash\left\{T_{k}\right\}$ and the configuration after $E^{\prime}$ is t-quiescent,

- every transaction $T_{m} \in \operatorname{txns}(E)$ that precedes $T_{k}$ in real-time order is included in $E^{\prime}$ such that $E\left|m=E^{\prime}\right| m$,

- for every transaction $T_{m} \in \operatorname{txns}\left(E^{\prime}\right), \operatorname{Rset}_{E^{\prime}}\left(T_{m}\right) \subseteq \operatorname{Rset}_{E}\left(T_{m}\right)$ and $\operatorname{Wset}_{E^{\prime}}\left(T_{m}\right) \subseteq \operatorname{Wset}_{E}\left(T_{m}\right)$, and

- $E^{\prime} \cdot E \mid k$ is an execution of $\mathcal{M}$.

Definition 7.1 (Data and metadata base objects). Let $\mathcal{X}$ be the set of t-objects operated by a HyTM implementation $\mathcal{M}$. Now we partition the set of base objects used by $\mathcal{M}$ into a set $\mathbb{D}$ of data objects and a set $\mathbb{M}$ of metadata objects $(\mathbb{D} \cap \mathbb{M}=\emptyset)$. We further partition $\mathbb{D}$ into sets $\mathbb{D}_{X}$ associated with each t-object $X \in \mathcal{X}: \mathbb{D}=\bigcup_{X \in \mathcal{X}} \mathbb{D}_{X}$, for all $X \neq Y$ in $\mathcal{X}, \mathbb{D}_{X} \cap \mathbb{D}_{Y}=\emptyset$, such that:

1. In every execution $E$, each fast-path transaction $T_{k} \in \operatorname{txns}(E)$ only accesses base objects in $\bigcup_{X \in D \operatorname{Set}\left(T_{k}\right)} \mathbb{D}_{X}$ or $\mathbb{M}$.

2. Let $E \cdot \rho$ and $E \cdot E^{\prime} \cdot \rho^{\prime}$ be two $t$-complete executions, such that $E$ and $E \cdot E^{\prime}$ are $t$-complete, $\rho$ and $\rho^{\prime}$ are complete executions of a transaction $T_{k} \notin \operatorname{txns}\left(E \cdot E^{\prime}\right), H_{\rho}=H_{\rho^{\prime}}$, and $\forall T_{m} \in \operatorname{txns}\left(E^{\prime}\right)$, $\operatorname{Dset}\left(T_{m}\right) \cap \operatorname{Dset}\left(T_{k}\right)=\emptyset$. Then the states of the base objects $\bigcup_{X \in D \operatorname{Set}\left(T_{k}\right)} \mathbb{D}_{X}$ in the configuration after $E \cdot \rho$ and $E \cdot E^{\prime} \cdot \rho^{\prime}$ are the same. 


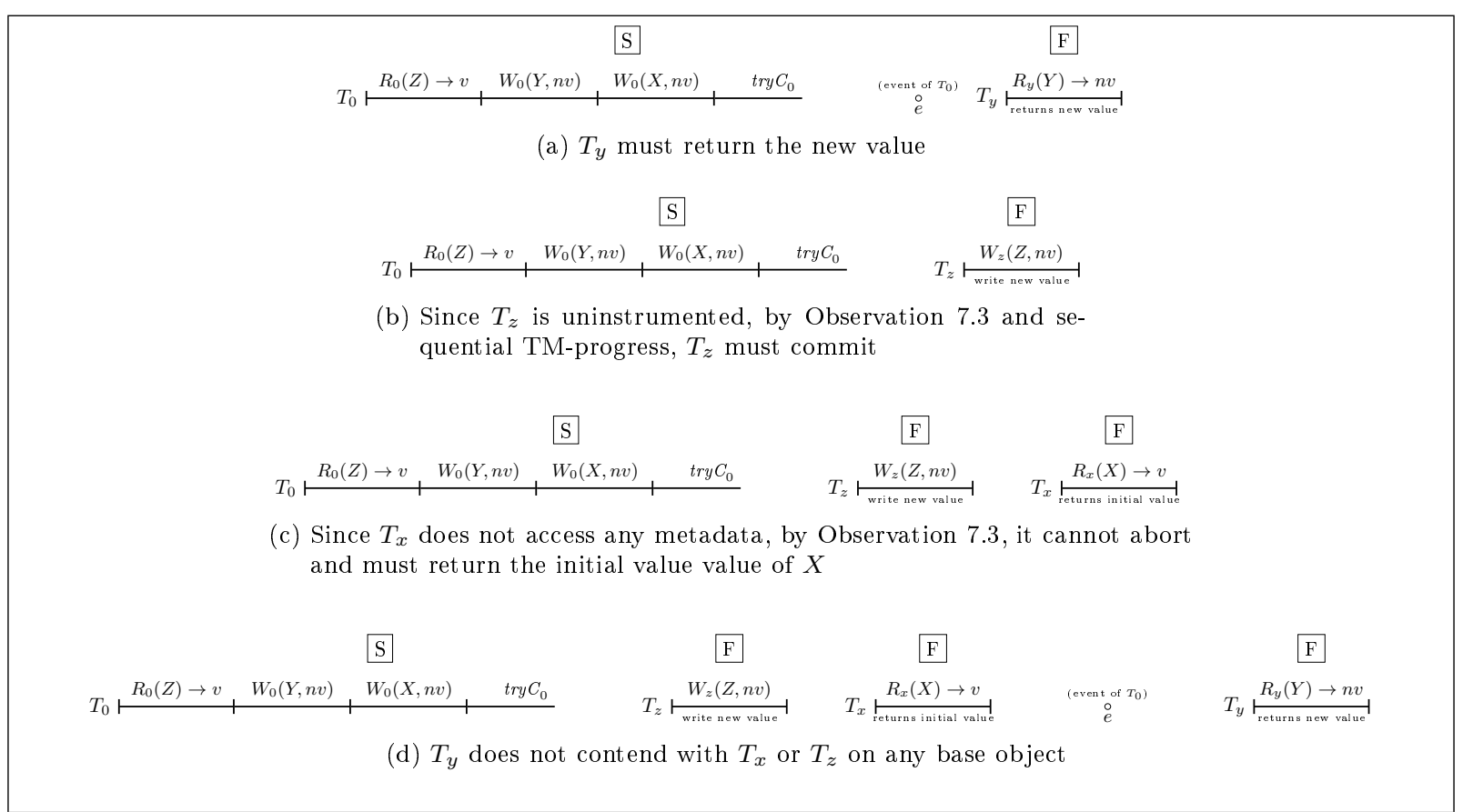

Figure 7.3: Executions in the proof of Theorem 7.4 execution in $7.3 \mathrm{~d}$ is not strictly serializable

3. Let execution $E$ appear t-sequential to a transaction $T_{k}$ and let the enabled event e of $T_{k}$ after $E$ be a primitive on a base object $b \in \mathbb{D}$. Then, unless e returns $\perp, E \cdot$ e also appears t-sequential to $T_{k}$.

Intuitively, the first condition says that a transaction is only allowed to access data objects based on its data set. The second condition says that transactions with disjoint data sets can communicate only via metadata objects. Finally, the last condition means that base objects in $\mathbb{D}$ may only contain the "values" of t-objects, and cannot be used to detect concurrent transactions. Note that our results will lower bound the number of metadata objects that must be accessed under particular assumptions, thus from a cost perspective, $\mathbb{D}$ should be made as large as possible.

All HyTM proposals we aware of, such as HybridNOrec [34, 109, PhTM [96] and others [36, 86, conform to our definition of instrumentation in fast-path transactions. For instance, HybridNOrec [34, 109] employs a distinct base object in $\mathbb{D}$ for each t-object and a global sequence lock as the metadata that is accessed by fast-path transactions to detect concurrency with slow-path transactions. Similarly, the HyTM implementation by Damron et al. [36] also associates a distinct base object in $\mathbb{D}$ for each t-object and additionally, a transaction header and ownership record as metadata base objects.

Definition 7.2 (Uninstrumented HyTMs). A HyTM implementation $\mathcal{M}$ provides uninstrumented writes (resp. reads) if in every execution $E$ of $\mathcal{M}$, for every write-only (resp. read-only) fast-path transaction $T_{k}$, all primitives in $E \mid k$ are performed on base objects in $\mathbb{D}$. A HyTM is uninstrumented if both its reads and writes are uninstrumented.

Observation 7.3. Consider any execution $E$ of a HyTM implementation $\mathcal{M}$ which provides uninstrumented reads (resp. writes). For any fast-path read-only (resp. write-only) transaction $T_{k} \notin \operatorname{txns}(E)$, that runs step-contention free after $E$, the execution $E$ appears $t$-sequential to $T_{k}$.

\subsubsection{Impossibility of uninstrumented HyTMs}

In this section, we show that any strictly serializable HyTM must be instrumented, even under a very weak progress assumption by which a transaction is guaranteed to commit only when run t-sequentially: 
Definition 7.3 (Sequential TM-progress for HyTMs). A HyTM implementation $\mathcal{M}$ provides sequential TM-progress for fast-path transactions (and resp. slow-path) if in every execution $E$ of $\mathcal{M}$, a fastpath (and resp. slow-path) transaction $T_{k}$ returns $A_{k}$ in $E$ only if $T_{k}$ incurs a capacity abort or $T_{k}$ is concurrent to another transaction. We say that $\mathcal{M}$ provides sequential TM-progress if it provides sequential TM-progress for fast-path and slow-path transactions.

Theorem 7.4. There does not exist a strictly serializable uninstrumented HyTM implementation that ensures sequential TM-progress and TM-liveness.

Proof. Suppose by contradiction that such a HyTM $\mathcal{M}$ exists. For simplicity, assume that $v$ is the initial value of t-objects $X, Y$ and $Z$. Let $E$ be the t-complete step contention-free execution of a slow-path transaction $T_{0}$ that performs $\operatorname{read}_{0}(Z) \rightarrow v$, write $(X, n v)$, write $e_{0}(Y, n v)(n v \neq v)$, and commits. Such an execution exists since $\mathcal{M}$ ensures sequential TM-progress.

By Observation 7.3 any transaction that runs step contention-free starting from a prefix of $E$ must return a non-abort value. Since any such transaction reading $X$ or $Y$ must return $v$ when it starts from the empty prefix of $E$ and $n v$ when it starts from $E$.

Thus, there exists $E^{\prime}$, the longest prefix of $E$ that cannot be extended with the t-complete step contentionfree execution of a fast-path transaction reading $X$ or $Y$ and returning $n v$. Let $e$ is the enabled event of $T_{0}$ in the configuration after $E^{\prime}$. Without loss of generality, suppose that there exists an execution $E^{\prime} \cdot e \cdot E_{y}$ where $E_{y}$ is the t-complete step contention-free execution fragment of some fast-path transaction $T_{y}$ that reads $Y$ is returns $n v$ (Figure 7.3a).

Claim 7.5. $\mathcal{M}$ has an execution $E^{\prime} \cdot E_{z} \cdot E_{x}$, where

- $E_{z}$ is the t-complete step contention-free execution fragment of a fast-path transaction $T_{z}$ that writes $n v \neq v$ to $Z$ and commits

- $E_{x}$ is the t-complete step contention-free execution fragment of a fast-path transaction $T_{x}$ that performs a single t-read read ${ }_{x}(X) \rightarrow v$ and commits.

Proof. By Observation 7.3 the extension of $E^{\prime}$ in which $T_{z}$ writes to $Z$ and tries to commit appears tsequential to $T_{z}$. By sequential TM-progress, $T_{z}$ complets the write and commits. Let $E^{\prime} \cdot E_{z}$ (Figure $7.3 \mathrm{~b}$ ) be the resulting execution of $\mathcal{M}$.

Similarly, the extension of $E^{\prime}$ in which $T_{x}$ reads $X$ and tries to commit appears t-sequential to $T_{x}$. By sequential TM-progress, $T_{x}$ commits and let $E^{\prime} \cdot E_{x}$ be the resulting execution of $\mathcal{M}$. By the definition of $E^{\prime}, \operatorname{read}_{x}(X)$ must return $v$ in $E^{\prime} \cdot E_{x}$.

Since $\mathcal{M}$ is uninstrumented and the data sets of $T_{x}$ and $T_{z}$ are disjoint, the sets of base objects accessed in the execution fragments $E_{x}$ and $E_{y}$ are also disjoint. Thus, $E^{\prime} \cdot E_{z} \cdot E_{x}$ is indistinguishable to $T_{x}$ from the execution $E^{\prime} \cdot E_{x}$, which implies that $E^{\prime} \cdot E_{z} \cdot E_{x}$ is an execution of $\mathcal{M}$ (Figure 7.3c).

Finally, we prove that the sequence of events, $E^{\prime} \cdot E_{z} \cdot E_{x} \cdot e \cdot E_{y}$ is an execution of $\mathcal{M}$.

Since the transactions $T_{x}, T_{y}, T_{z}$ have pairwise disjoint data sets in $E^{\prime} \cdot E_{z} \cdot E_{x} \cdot e \cdot E_{y}$, no base object accessed ib $E_{y}$ can be accessed in $E_{x}$ and $E_{z}$. The read operation on $X$ performed by $T_{y}$ in $E^{\prime} \cdot e \cdot E_{y}$ returns $n v$ and, by the definition of $E^{\prime}$ and $e, T_{y}$ must have accessed the base object $b$ modified in the event $e$ by $T_{0}$. Thus, $b$ is not accessed in $E_{x}$ and $E_{z}$ and $E^{\prime} \cdot E_{z} \cdot E_{x} \cdot e$ is an execution of $\mathcal{M}$. Summing up, $E^{\prime} \cdot E_{z} \cdot E_{x} \cdot e \cdot E_{y}$ is indistinguishable to $T_{y}$ from $E^{\prime} \cdot e \cdot E_{y}$, which implies that $E^{\prime} \cdot E_{z} \cdot E_{x} \cdot e \cdot E_{y}$ is an execution of $\mathcal{M}$ (Figure $7.3 \mathrm{~d}$ ).

But the resulting execution is not strictly serializable. Indeed, suppose that a serialization exists. As the value written by $T_{0}$ is returned by a committed transaction $T_{y}, T_{0}$ must be committed and precede $T_{y}$ in the serialization. Since $T_{x}$ returns the initial value of $X, T_{x}$ must precede $T_{0}$. Since $T_{0}$ reads the initial value of $Z, T_{0}$ must precede $T_{z}$. Finally, $T_{z}$ must precede $T_{x}$ to respect the real-time order. The cycle in the serialization establishes a contradiction. 


\subsection{A linear lower bound on instrumentation for progressive HyTMs}

In this section, we show that giving HyTM the ability to run and commit transactions in parallel brings considerable instrumentation costs. We focus on a natural progress condition called progressiveness [59, 60. 61 that allows a transaction to abort only if it experiences a read-write or write-write conflict with a concurrent transaction:

Definition 7.4 (Progressiveness for HyTMs). We say that transactions $T_{i}$ and $T_{j}$ conflict in an execution $E$ on a $t$-object $X$ if $X \in \operatorname{Dset}\left(T_{i}\right) \cap \operatorname{Dset}\left(T_{j}\right)$ and $X \in W \operatorname{set}\left(T_{i}\right) \cup W \operatorname{set}\left(T_{j}\right)$.

A HyTM implementation $\mathcal{M}$ is fast-path (resp. slow-path) progressive if in every execution $E$ of $\mathcal{M}$ and for every fast-path (and resp. slow-path) transaction $T_{i}$ that aborts in $E$, either $A_{i}$ is a capacity abort or $T_{i}$ conflicts with some transaction $T_{j}$ that is concurrent to $T_{i}$ in $E$. We say $\mathcal{M}$ is progressive if it is both fast-path and slow-path progressive.

We show that for every opaque fast-path progressive HyTM that provides obstruction-free TM-liveness, an arbitrarily long read-only transaction might access a number of distinct metadata base objects that is linear in the size of its read set or experience a capacity abort.

The following auxiliary results will be crucial in proving our lower bound. We observe first that a fast path transaction in a progressive HyTM can contend on a base object only with a conflicting transaction.

Lemma 7.6. Let $\mathcal{M}$ be any fast-path progressive HyTM implementation. Let $E \cdot E_{1} \cdot E_{2}$ be an execution of $\mathcal{M}$ where $E_{1}$ (and resp. $E_{2}$ ) is the step contention-free execution fragment of transaction $T_{1} \notin$ txns $(E)$ (and resp. $T_{2} \notin$ txns $(E)$ ), $T_{1}$ (and resp. $T_{2}$ ) does not conflict with any transaction in $E \cdot E_{1} \cdot E_{2}$, and at least one of $T_{1}$ or $T_{2}$ is a fast-path transaction. Then, $T_{1}$ and $T_{2}$ do not contend on any base object in $E \cdot E_{1} \cdot E_{2}$.

Proof. Suppose, by contradiction that $T_{1}$ or $T_{2}$ contend on the same base object in $E \cdot E_{1} \cdot E_{2}$.

If in $E_{1}, T_{1}$ performs a nontrivial event on a base object on which they contend, let $e_{1}$ be the last event in $E_{1}$ in which $T_{1}$ performs such an event to some base object $b$ and $e_{2}$, the first event in $E_{2}$ that accesses $b$. Otherwise, $T_{1}$ only performs trivial events in $E_{1}$ to base objects on which it contends with $T_{2}$ in $E \cdot E_{1} \cdot E_{2}$ : let $e_{2}$ be the first event in $E_{2}$ in which $E_{2}$ performs a nontrivial event to some base object $b$ on which they contend and $e_{1}$, the last event of $E_{1}$ in $T_{1}$ that accesses $b$.

Let $E_{1}^{\prime}$ (and resp. $E_{2}^{\prime}$ ) be the longest prefix of $E_{1}$ (and resp. $E_{2}$ ) that does not include $e_{1}$ (and resp. $\left.e_{2}\right)$. Since before accessing $b$, the execution is step contention-free for $T_{1}, E \cdot E_{1}^{\prime} \cdot E_{2}^{\prime}$ is an execution of $\mathcal{M}$. By construction, $T_{1}$ and $T_{2}$ do not conflict in $E \cdot E_{1}^{\prime} \cdot E_{2}^{\prime}$. Moreover, $E \cdot E_{1} \cdot E_{2}^{\prime}$ is indistinguishable to $T_{2}$ from $E \cdot E_{1}^{\prime} \cdot E_{2}^{\prime}$. Hence, $T_{1}$ and $T_{2}$ are poised to apply contending events $e_{1}$ and $e_{2}$ on $b$ in the execution $\tilde{E}=E \cdot E_{1}^{\prime} \cdot E_{2}^{\prime}$. Recall that at least one event of $e_{1}$ and $e_{2}$ must be nontrivial.

Consider the execution $\tilde{E} \cdot e_{1} \cdot e_{2}^{\prime}$ where $e_{2}^{\prime}$ is the event of $p_{2}$ in which it applies the primitive of $e_{2}$ to the configuration after $\tilde{E} \cdot e_{1}$. After $\tilde{E} \cdot e_{1}, b$ is contained in the tracking set of process $p_{1}$. If $b$ is contained in $\tau_{1}$ in the shared mode, then $e_{2}^{\prime}$ is a nontrivial primitive on $b$, which invalidates $\tau_{1}$ in $\tilde{E} \cdot e_{1} \cdot e_{2}^{\prime}$. If $b$ is contained in $\tau_{1}$ in the exclusive mode, then any subsequent access of $b$ invalidates $\tau_{1}$ in $\tilde{E} \cdot e_{1} \cdot e_{2}^{\prime}$. In both cases, $\tau_{1}$ is invalidated and $T_{1}$ incurs a tracking set abort. Thus, transaction $T_{1}$ must return $A_{1}$ in any extension of $E \cdot e_{1} \cdot e_{2}-$ a contradiction to the assumption that $\mathcal{M}$ is progressive.

Iterative application of Lemma 7.6 implies the following:

Corollary 7.7. Let $\mathcal{M}$ be any fast-path progressive HyTM implementation. Let $E \cdot E_{1} \cdots E_{i} \cdot E_{i+1} \cdots E_{m}$ be any execution of $\mathcal{M}$ where for all $i \in\{1, \ldots, m\}, E_{i}$ is the step contention-free execution fragment of transaction $T_{i} \notin$ txns $(E)$ and any two transactions in $E_{1} \cdots E_{m}$ do not conflict. For all $i, j=1, \ldots, m$, $i \neq j$, if $T_{i}$ is fast-path, then $T_{i}$ and $T_{j}$ do not contend on a base object in $E \cdot E_{1} \cdots E_{i} \cdots E_{m}$ 
Proof. Let $T_{i}$ be a fast-path transaction. By Lemma 7.6 in $E \cdot E_{1} \cdots E_{i} \cdots E_{m}, T_{i}$ does not contend with $T_{i-1}\left(\right.$ if $i>1$ ) or $T_{i+1}$ (if $i<m$ ) on any base object and, thus, $E_{i}$ commutes with $E_{i-1}$ and $E_{i+1}$. Thus, $E \cdot E_{1} \cdots E_{i-2} \cdot E_{i} \cdot E_{i-1} \cdot E_{i+1} \cdots E_{m}$ (if $i>1$ ) and $E \cdot E_{1} \cdots E_{i-1} \cdot E_{i+1} \cdot E_{i} \cdot E_{i+2} \cdots E_{m}$ (if $i<m$ ) are executions of $\mathcal{M}$. By iteratively applying Lemma 7.6, we derive that $T_{i}$ does not contend with any $T_{j}, j \neq i$.

We say that execution fragments $E$ and $E^{\prime}$ are similar if they export equivalent histories, i.e., no process can see the difference between them by looking at the invocations and responses of t-operations. We now use Corollary 7.7 to show that t-operations only accessing data base objects cannot detect contention with non-conflicting transactions.

Lemma 7.8. Let $E$ be any t-complete execution of a progressive HyTM implementation $\mathcal{M}$ that provides OF TM-liveness. For any $m \in \mathbb{N}$, consider a set of $m$ executions of $\mathcal{M}$ of the form $E \cdot E_{i} \cdot \gamma_{i} \cdot \rho_{i}$ where $E_{i}$ is the t-complete step contention-free execution fragment of a transaction $T_{m+i}, \gamma_{i}$ is a complete step contention-free execution fragment of a fast-path transaction $T_{i}$ such that $\operatorname{Dset}\left(T_{i}\right) \cap \operatorname{Dset}\left(T_{m+i}\right)=\emptyset$ in $E \cdot E_{i} \cdot \gamma_{i}$, and $\rho_{i}$ is the execution fragment of a $t$-operation by $T_{i}$ that does not contain accesses to any metadata base object. If, for all $i, j \in\{1, \ldots, m\}, i \neq j, \operatorname{Dset}\left(T_{i}\right) \cap \operatorname{Dset}\left(T_{m+j}\right)=\emptyset, \operatorname{Dset}\left(T_{i}\right) \cap \operatorname{Dset}\left(T_{j}\right)=$ $\emptyset$ and Dset $\left(T_{m+i}\right) \cap D$ set $\left(T_{m+j}\right)=\emptyset$, then there exists a $t$-complete step contention-free execution fragment $E^{\prime}$ that is similar to $E_{1} \cdots E_{m}$ such that for all $i \in\{1, \ldots, m\}, E \cdot E^{\prime} \cdot \gamma_{i} \cdot \rho_{i}$ is an execution of $\mathcal{M}$.

Proof. Observe that any two transactions in the execution fragment $E_{1} \cdots E_{m}$ access mutually disjoint data sets. Since $\mathcal{M}$ is progressive and provides OF TM-liveness, there exists a t-sequential execution fragment $E^{\prime}=E_{1}^{\prime} \cdots E_{m}^{\prime}$ such that, for all $i \in\{1, \ldots, m\}$, the execution fragments $E_{i}$ and $E_{i}^{\prime}$ are similar and $E \cdot E^{\prime}$ is an execution of $\mathcal{M}$. Corollary 7.7 implies that, for all for all $i \in\{1, \ldots, m\}, \mathcal{M}$ has an execution of the form $E \cdot E_{1}^{\prime} \cdots E_{i}^{\prime} \cdots E_{m}^{\prime} \cdot \gamma_{i}$. More specifically, $\mathcal{M}$ has an execution of the form $E \cdot \gamma_{i} \cdot E_{1}^{\prime} \cdots E_{i}^{\prime} \cdots E_{m}^{\prime}$. Recall that the execution fragment $\rho_{i}$ of fast-path transaction $T_{i}$ that extends $\gamma_{i}$ contains accesses only to base objects in $\bigcup_{X \in D \operatorname{Set}\left(T_{i}\right)} \mathbb{D}_{X}$. Moreover, for all $i, j \in\{1, \ldots, m\} ; i \neq j$, $\operatorname{Dset}\left(T_{i}\right) \cap \operatorname{Dset}\left(T_{m+j}\right)=\emptyset$ and $\operatorname{Dset}\left(T_{m+i}\right) \cap \operatorname{Dset}\left(T_{m+j}\right)=\emptyset$.

It follows that $\mathcal{M}$ has an execution of the form $E \cdot \gamma_{i} \cdot E_{1}^{\prime} \cdots E_{i}^{\prime} \cdot \rho_{i} \cdot E_{i+1}^{\prime} \cdots E_{m}^{\prime}$. and the states of each of the base objects $\bigcup_{X \in D \operatorname{Set}\left(T_{i}\right)} \mathbb{D}_{X}$ accessed by $T_{i}$ in the configuration after $E \cdot \gamma_{i} \cdot E_{1}^{\prime} \cdots E_{i}^{\prime}$ and $E \cdot \gamma_{i} \cdot E_{i}$ are the same. But $E \cdot \gamma_{i} \cdot E_{i} \cdot \rho_{i}$ is an execution of $\mathcal{M}$. Thus, for all $i \in\{1, \ldots, m\}, \mathcal{M}$ has an execution of the form $E \cdot E^{\prime} \cdot \gamma_{i} \cdot \rho_{i}$.

Finally, we are now ready to derive our lower bound.

Theorem 7.9. Let $\mathcal{M}$ be any progressive, opaque HyTM implementation that provides OF TM-liveness. For every $m \in \mathbb{N}$, there exists an execution $E$ in which some fast-path read-only transaction $T_{k} \in$ txns $(E)$ satisfies either (1) Dset $\left(T_{k}\right) \leq m$ and $T_{k}$ incurs a capacity abort in $E$ or (2) Dset $\left(T_{k}\right)=m$ and $T_{k}$ accesses $\Omega(m)$ distinct metadata base objects in $E$.

Here is a high-level overview of the proof technique. Let $\kappa$ be the smallest integer such that some fastpath transaction running step contention-free after a t-quiescent configuration performs $\kappa$ t-reads and incurs a capacity abort.

We prove that, for all $m \leq \kappa-1$, there exists a t-complete execution $E_{m}$ and a set $S_{m}$ with $\left|S_{m}\right|=2^{\kappa-m}$ of read-only fast-path transactions that access mutually disjoint data sets such that each transaction in $S_{m}$ that runs step contention-free from $E_{m}$ and performs t-reads of $m$ distinct t-objects accesses at least one distinct metadata base object within the execution of each t-read operation.

We proceed by induction. Assume that the induction statement holds for all $m<k a p p a-1$. We prove that a set $S_{m+1} ;\left|S_{m+1}\right|=2^{\kappa-(m+1)}$ of fast-path transactions, each of which run step contention-free after the same t-complete execution $E_{m+1}$, perform $m+1$ t-reads of distinct t-objects so that at least one distinct metadata base object is accessed within the execution of each t-read operation. In our construction, we pick any two new transactions from the set $S_{m}$ and show that one of them running 
step contention-free from a t-complete execution that extends $E_{m}$ performs $m+1$ t-reads of distinct t-objects so that at least one distinct metadata base object is accessed within the execution of each t-read operation. In this way, the set of transactions is reduced by half in each step of the induction until one transaction remains which must have accessed a distinct metadata base object in every one of its $m+1$ t-reads.

Intuitively, since all the transactions that we use in our construction access mutually disjoint data sets, we can apply Lemma 7.6 to construct a t-complete execution $E_{m+1}$ such that each of the fast-path transactions in $S_{m+1}$ when running step contention-free after $E_{m+1}$ perform $m+1$ t-reads so that at least one distinct metadata base object is accessed within the execution of each t-read operation.

We now present the formal proof:

Proof. In the constructions which follow, every fast-path transaction executes at most $m+1$ t-reads. Let $\kappa$ be the smallest integer such that some fast-path transaction running step contention-free after a t-quiescent configuration performs $\kappa$ t-reads and incurs a capacity abort. We proceed by induction.

Induction statement. We prove that, for all $m \leq \kappa-1$, there exists a t-complete execution $E_{m}$ and a set $S_{m}$ with $\left|S_{m}\right|=2^{\kappa-m}$ of read-only fast-path transactions that access mutually disjoint data sets such that each transaction $T_{f_{i}} \in S_{m}$ that runs step contention-free from $E_{m}$ and performs t-reads of $m$ distinct t-objects accesses at least one distinct metadata base object within the execution of each t-read operation. Let $E_{f_{i}}$ be the step contention-free execution of $T_{f_{i}}$ after $E_{m}$ and let $\operatorname{Dset}\left(T_{f_{i}}\right)=\left\{X_{i, 1}, \ldots, X_{i, m}\right\}$.

The induction. Assume that the induction statement holds for all $m \leq \kappa-1$. The statement is trivially true for the base case $m=0$ for every $\kappa \in \mathbb{N}$.

We will prove that a set $S_{m+1} ;\left|S_{m+1}\right|=2^{\kappa-(m+1)}$ of fast-path transactions, each of which run step contention-free from the same t-quiescent configuration $E_{m+1}$, perform $m+1$ t-reads of distinct tobjects so that at least one distinct metadata base object is accessed within the execution of each t-read operation.

The construction proceeds in phases: there are exactly $\frac{\left|S_{m}\right|}{2}$ phases. In each phase, we pick any two new transactions from the set $S_{m}$ and show that one of them running step contention-free after a t-complete execution that extends $E_{m}$ performs $m+1$ t-reads of distinct t-objects so that at least one distinct metadata base object is accessed within the execution of each t-read operation.

Throughout this proof, we will assume that any two transactions (and resp. execution fragments) with distinct subscripts represent distinct identifiers.

For all $i \in\left\{0, \ldots, \frac{\left|S_{m}\right|}{2}-1\right\}$, let $X_{2 i+1}, X_{2 i+2} \notin \bigcup_{i=0}^{\left|S_{m}\right|-1}\left\{X_{i, 1}, \ldots, X_{i, m}\right\}$ be distinct t-objects and let $v$ be the value of $X_{2 i+1}$ and $X_{2 i+2}$ after $E_{m}$. Let $T_{s_{i}}$ denote a slow-path transaction which writes $n v \neq v$ to $X_{2 i+1}$ and $X_{2 i+2}$. Let $E_{s_{i}}$ be the t-complete step contention-free execution fragment of $T_{s_{i}}$ running immediately after $E_{m}$.

Let $E_{s_{i}}^{\prime}$ be the longest prefix of the execution $E_{s_{i}}$ such that $E_{m} \cdot E_{s_{i}}^{\prime}$ can be extended neither with the complete step contention-free execution fragment of transaction $T_{f_{2 i+1}}$ that performs its $m$ t-reads of $X_{2 i+1,1}, \ldots, X_{2 i+1, m}$ and then performs $\operatorname{read}_{f_{2 i+1}}\left(X_{2 i+1}\right)$ and returns $n v$, nor with the complete step contention-free execution fragment of some transaction $T_{f_{2 i+2}}$ that performs t-reads of $X_{2 i+2_{1}}, \ldots, X_{2 i+2, m}$ and then performs $\operatorname{read}_{f_{2 i+2}}\left(X_{2 i+2}\right)$ and returns $n v$. Progressiveness and OF TM-liveness of $\mathcal{M}$ stipulates that such an execution exists.

Let $e_{i}$ be the enabled event of $T_{s_{i}}$ in the configuration after $E_{m} \cdot E_{s_{i}}^{\prime}$. By construction, the execution $E_{m} \cdot E_{s_{i}}^{\prime}$ can be extended with at least one of the complete step contention-free executions of transaction $T_{f_{2 i+1}}$ performing $(m+1)$ t-reads of $X_{2 i+1,1}, \ldots, X_{2 i+1, m}, X_{2 i+1}$ such that read $_{f_{2 i+1}}\left(X_{2 i+1}\right) \rightarrow n v$ or transaction $T_{f_{2 i+2}}$ performing t-reads of $X_{2 i+2,1}, \ldots, X_{2 i+2, m}, X_{2 i+2}$ such that $\operatorname{read}_{f_{2 i+2}}\left(X_{2 i+2}\right) \rightarrow n v$. Without loss of generality, suppose that $T_{f_{2 i+1}}$ reads the value of $X_{2 i+1}$ to be $n v$ after $E_{m} \cdot E_{0_{i}}^{\prime} \cdot e_{i}$. 
For any $i \in\left\{0, \ldots, \frac{\left|S_{m}\right|}{2}-1\right\}$, we will denote by $\alpha_{i}$ the execution fragment which we will construct in phase $i$. For any $i \in\left\{0, \ldots, \frac{\left|S_{m}\right|}{2}-1\right\}$, we prove that $\mathcal{M}$ has an execution of the form $E_{m} \cdot \alpha_{i}$ in which $T_{f_{2 i+1}}$ (or $T_{f_{2 i+2}}$ ) running step contention-free after a t-complete execution that extends $E_{m}$ performs $m+1$ t-reads of distinct t-objects so that at least one distinct metadata base object is accessed within the execution of each first $m$ t-read operations and $T_{f_{2 i+1}}\left(\right.$ or $T_{f_{2 i+2}}$ ) is poised to apply an event after $E_{m} \cdot \alpha_{i}$ that accesses a distinct metadata base object during the $(m+1)^{t h}$ t-read. Furthermore, we will show that $E_{m} \cdot \alpha_{i}$ appears t-sequential to $T_{f_{2 i+1}}\left(\right.$ or $\left.T_{f_{2 i+2}}\right)$.

\section{(Construction of phase $i$ )}

Let $E_{f_{2 i+1}}$ (and resp. $E_{f_{2 i+2}}$ ) be the complete step contention-free execution of the t-reads of $X_{2 i+1,1}, \ldots, X_{2 i+1, m}$ (and resp. $\left.X_{2 i+2,1}, \ldots, X_{2 i+2, m}\right)$ running after $E_{m}$ by $T_{f_{2 i+1}}$ (and resp. $T_{f_{2 i+2}}$ ). By the inductive hypothesis, transaction $T_{f_{2 i+1}}$ (and resp. $T_{f_{2 i+2}}$ ) accesses $m$ distinct metadata objects in the execution $E_{m} \cdot E_{f_{2 i+1}}$ (and resp. $E_{m} \cdot E_{f_{2 i+2}}$ ). Recall that transaction $T_{f_{2 i+1}}$ does not conflict with transaction $T_{s_{i}}$. Thus, by Corollary 7.7, $\mathcal{M}$ has an execution of the form $E_{m} \cdot E_{s_{i}}^{\prime} \cdot e_{i} \cdot E_{f_{2 i+1}}$ (and resp. $E_{m} \cdot E_{s_{i}}^{\prime} \cdot e_{i} \cdot E_{f_{2 i+2}}$ ).

Let $E_{r f_{2 i+1}}$ be the complete step contention-free execution fragment of $\operatorname{read}_{f_{2 i+1}}\left(X_{2 i+1}\right)$ that extends $E_{2 i+1}=E_{m} \cdot E_{s_{i}}^{\prime} \cdot e_{i} \cdot E_{f_{2 i+1}}$. By OF TM-liveness, read $f_{2 i+1}\left(X_{2 i+1}\right)$ must return a matching response in $E_{2 i+1} \cdot E_{r f_{2 i+1}}$. We now consider two cases.

Case I: Suppose $E_{r f_{2 i+1}}$ accesses at least one metadata base object b not previously accessed by $T_{f_{2 i+1}}$.

Let $E_{r f_{2 i+1}}^{\prime}$ be the longest prefix of $E_{r f_{2 i+1}}$ which does not apply any primitives to any metadata base object $b$ not previously accessed by $T_{f_{2 i+1}}$. The execution $E_{m} \cdot E_{s_{i}}^{\prime} \cdot e_{i} \cdot E_{f_{2 i+1}} \cdot E_{r f_{2 i+1}}^{\prime}$ appears t-sequential to $T_{f_{2 i+1}}$ because $E_{f_{2 i+1}}$ does not contend with $T_{s_{i}}$ on any base object and any common base object accessed in the execution fragments $E_{r x_{2 i+1}}^{\prime}$ and $E_{s_{i}}$ by $T_{f_{2 i+1}}$ and $T_{s_{i}}$ respectively must be data objects contained in $\mathbb{D}$. Thus, we have that $\left|\operatorname{Dset}\left(T_{f_{2 i+1}}\right)\right|=m+1$ and that $T_{f_{2 i+1}}$ accesses $m$ distinct metadata base objects within each of its first $m$ t-read operations and is poised to access a distinct metadata base object during the execution of the $(m+1)^{t h}$ t-read. In this case, let $\alpha_{i}=E_{m} \cdot E_{s_{i}}^{\prime} \cdot e_{i} \cdot E_{f_{2 i+1}} \cdot E_{r f_{2 i+1}}^{\prime}$.

Case II: Suppose $E_{f_{2 i+1}}$ does not access any metadata base object not previously accessed by $T_{f_{2 i+1}}$.

In this case, we will first prove the following:

Claim 7.10. $\mathcal{M}$ has an execution of the form $E_{2 i+2}=E_{m} \cdot E_{s_{i}}^{\prime} \cdot e_{i} \cdot \bar{E}_{f_{2 i+1}} \cdot E_{f_{2 i+2}}$ where $\bar{E}_{f_{2 i+1}}$ is the t-complete step contention-free execution of $T_{f_{2 i+1}}$ in which read $f_{f_{2 i+1}}\left(X_{2 i+1}\right) \rightarrow$ nv, $T_{f_{2 i+1}}$ invokes try $C_{f_{2 i+1}}$ and returns a matching response.

Proof. Since $E_{r f_{2 i+1}}$ does not contain accesses to any distinct metadata base objects, the execution $E_{m} \cdot E_{s_{i}}^{\prime} \cdot e_{i} \cdot E_{f_{2 i+1}} \cdot E_{r f_{2 i+1}}$ appears t-sequential to $T_{f_{2 i+1}}$. By definition of the event $e_{i}, \operatorname{read}_{f_{2 i+1}}\left(X_{2 i+1}\right)$ must access the base object to which the event $e_{i}$ applies a nontrivial primitive and return the response $n v$ in $E_{s_{i}}^{\prime} \cdot e_{i} \cdot E_{f_{2 i+1}} \cdot E_{r f_{2 i+1}}$. By OF TM-liveness, it follows that $E_{m} \cdot E_{s_{i}}^{\prime} \cdot e_{i} \cdot \bar{E}_{f_{2 i+1}}$ is an execution of $\mathcal{M}$.

Now recall that $E_{m} \cdot E_{s_{i}}^{\prime} \cdot e_{i} \cdot E_{f_{2 i+2}}$ is an execution of $\mathcal{M}$ because transactions $T_{f_{2 i+2}}$ and $T_{s_{i}}$ do not conflict in this execution and thus, cannot contend on any base object. Finally, because $T_{f_{2 i+1}}$ and $T_{f_{2 i+2}}$ access disjoint data sets in $E_{m} \cdot E_{s_{i}}^{\prime} \cdot e_{i} \cdot \bar{E}_{f_{2 i+1}} \cdot E_{f_{2 i+2}}$, by Lemma 7.6 again, we have that $E_{m} \cdot E_{s_{i}}^{\prime} \cdot e_{i} \cdot \bar{E}_{f_{2 i+1}} \cdot E_{f_{2 i+2}}$ is an execution of $\mathcal{M}$.

Let $E_{r f_{2 i+2}}$ be the complete step contention-free execution fragment of $\operatorname{read}_{f_{2 i+2}}\left(X_{2 i+2}\right)$ after $E_{m} \cdot E_{s_{i}}^{\prime}$. $e_{i} \cdot \bar{E}_{f_{2 i+1}} \cdot E_{f_{2 i+2}}$. By the induction hypothesis and Claim 7.10 transaction $T_{f_{2 i+2}}$ must access $m$ distinct metadata base objects in the execution $E_{m} \cdot E_{s_{i}}^{\prime} \cdot e_{i} \cdot \bar{E}_{f_{2 i+1}} \cdot E_{f_{2 i+2}}$.

If $E_{r f_{2 i+2}}$ accesses some metadata base object, then by the argument given in Case I applied to transaction $T_{f_{2 i+2}}$, we get that $T_{f_{2 i+2}}$ accesses $m$ distinct metadata base objects within each of the first $m$ t-read operations and is poised to access a distinct metadata base object during the execution of the $(m+1)^{t h}$ t-read. 
Thus, suppose that $E_{r f_{2 i+2}}$ does not access any metadata base object previously accessed by $T_{f_{2 i+2}}$. We claim that this is impossible and proceed to derive a contradiction. In particular, $E_{r f_{2 i+2}}$ does not contend with $T_{s_{i}}$ on any metadata base object. Consequently, the execution $E_{m} \cdot E_{s_{i}}^{\prime} \cdot e_{i} \cdot \bar{E}_{f_{2 i+1}} \cdot E_{f_{2 i+2}}$ appears t-sequential to $T_{x_{2 i+2}}$ since $E_{r x_{2 i+2}}$ only contends with $T_{s_{i}}$ on base objects in $\mathbb{D}$. It follows that $E_{2 i+2} \cdot E_{r f_{2 i+2}}$ must also appear t-sequential to $T_{f_{2 i+2}}$ and so $E_{r f_{2 i+2}}$ cannot abort. Recall that the base object, say $b$, to which $T_{s_{i}}$ applies a nontrivial primitive in the event $e_{i}$ is accessed by $T_{f_{2 i+1}}$ in $E_{m} \cdot E_{s_{i}}^{\prime} \cdot e_{i} \cdot \bar{E}_{f_{2 i+1}} \cdot E_{f_{2 i+2}} ;$ thus, $b \in \mathbb{D}_{X_{2 i+1}}$. Since $X_{2 i+1} \notin \operatorname{Dset}\left(T_{f_{2 i+2}}\right), b$ cannot be accessed by $T_{f_{2 i+2}}$. Thus, the execution $E_{m} \cdot E_{s_{i}}^{\prime} \cdot e_{i} \cdot \bar{E}_{f_{2 i+1}} \cdot E_{f_{2 i+2}} \cdot E_{r f_{2 i+2}}$ is indistinguishable to $T_{f_{2 i+2}}$ from the execution $\hat{E}_{i} \cdot E_{s_{i}}^{\prime} \cdot E_{f_{2 i+2}} \cdot E_{r f_{2 i+2}}$ in which $\operatorname{read}_{f_{2 i+2}}\left(X_{2 i+2}\right)$ must return the response $v$ (by construction of $E_{s_{i}}^{\prime}$ ).

But we observe now that the execution $E_{m} \cdot E_{s_{i}}^{\prime} \cdot e_{i} \cdot \bar{E}_{f_{2 i+1}} \cdot E_{f_{2 i+2}} \cdot E_{r f_{2 i+2}}$ is not opaque. In any serialization corresponding to this execution, $T_{s_{i}}$ must be committed and must precede $T_{f_{2 i+1}}$ because $T_{f_{2 i+1}}$ read $n v$ from $X_{2 i+1}$. Also, transaction $T_{f_{2 i+2}}$ must precede $T_{s_{i}}$ because $T_{f_{2 i+2}}$ read $v$ from $X_{2 i+2}$. However $T_{f_{2 i+1}}$ must precede $T_{f_{2 i+2}}$ to respect real-time ordering of transactions. Clearly, there exists no such serialization-contradiction.

Letting $E_{r f_{2 i+2}}^{\prime}$ be the longest prefix of $E_{r f_{2 i+2}}$ which does not access a base object $b \in \mathbb{M}$ not previously accessed by $T_{f_{2 i+2}}$, we can let $\alpha_{i}=E_{s_{i}}^{\prime} \cdot e_{i} \cdot \bar{E}_{f_{2 i+1}} \cdot E_{f_{2 i+2}} \cdot E_{r f_{2 i+2}}^{\prime}$ in this case.

Combining Cases I and II, the following claim holds.

Claim 7.11. For each $i \in\left\{0, \ldots, \frac{\left|S_{m}\right|}{2}-1\right\}, \mathcal{M}$ has an execution of the form $E_{m} \cdot \alpha_{i}$ in which

(1) some fast-path transaction $T_{i} \in$ txns $\left(\alpha_{i}\right)$ performs $t$-reads of $m+1$ distinct $t$-objects so that at least one distinct metadata base object is accessed within the execution of each of the first $m$ t-reads, $T_{i}$ is poised to access a distinct metadata base object after $E_{m} \cdot \alpha_{i}$ during the execution of the $(m+1)^{\text {th }}$ t-read and the execution appears t-sequential to $T_{i}$,

(2) the two fast-path transactions in the execution fragment $\alpha_{i}$ do not contend on the same base object.

\section{(Collecting the phases)}

We will now describe how we can construct the set $S_{m+1}$ of fast-path transactions from these $\frac{\left|S_{m}\right|}{2}$ phases and force each of them to access $m+1$ distinct metadata base objects when running step contention-free after the same t-complete execution.

For each $i \in\left\{0, \ldots, \frac{\left|S_{m}\right|}{2}-1\right\}$, let $\beta_{i}$ be the subsequence of the execution $\alpha_{i}$ consisting of all the events of the fast-path transaction that is poised to access a $(m+1)^{t h}$ distinct metadata base object. Henceforth, we denote by $T_{i}$ the fast-path transaction that participates in $\beta_{i}$. Then, from Claim 7.11 it follows that, for each $i \in\left\{0, \ldots, \frac{\left|S_{m}\right|}{2}-1\right\}, \mathcal{M}$ has an execution of the form $E_{m} \cdot E_{s_{i}}^{\prime} \cdot e_{i} \cdot \beta_{i}$ in which the fast-path transaction $T_{i}$ performs t-reads of $m+1$ distinct t-objects so that at least one distinct metadata base object is accessed within the execution of each of the first $m$ t-reads, $T_{i}$ is poised to access a distinct metadata base object after $E_{m} \cdot E_{s_{i}}^{\prime} \cdot e_{i} \cdot \beta_{i}$ during the execution of the $(m+1)^{t h}$ t-read and the execution appears t-sequential to $T_{i}$.

The following result is a corollary to the above claim that is obtained by applying the definition of "appears t-sequential". Recall that $E_{s_{i}}^{\prime} \cdot e_{i}$ is the t-incomplete execution of slow-path transaction $T_{s_{i}}$ that accesses t-objects $X_{2 i+1}$ and $X_{2 i+2}$.

Corollary 7.12. For all $i \in\left\{0, \ldots, \frac{\mid\left(S_{m} \mid\right.}{2}-1\right\}, \mathcal{M}$ has an execution of the form $E_{m} \cdot E_{i} \cdot \beta_{i}$ such that the configuration after $E_{m} \cdot E_{i}$ is t-quiescent, txns $\left(E_{i}\right) \subseteq\left\{T_{s_{i}}\right\}$ and $\operatorname{Dset}\left(T_{s_{i}}\right) \subseteq\left\{X_{2 i+1}, X_{2 i+2}\right\}$ in $E_{i}$.

We can represent the execution $\beta_{i}=\gamma_{i} \cdot \rho_{i}$ where fast-path transaction $T_{i}$ performs complete t-reads of $m$ distinct t-objects in $\gamma_{i}$ and then performs an incomplete t-read of the $(m+1)^{t h}$ t-object in $\rho_{i}$ in which $T_{i}$ only accesses base objects in $\bigcup\{X\}$. Recall that $T_{i}$ and $T_{s_{i}}$ do not contend on the same base $X \in D \operatorname{Set}\left(T_{i}\right)$

object in the execution $E_{m} \cdot E_{i} \cdot \gamma_{i}$. Thus, for all $i \in\left\{0, \ldots, \frac{\left|S_{m}\right|}{2}-1\right\}, \mathcal{M}$ has an execution of the form $E_{m} \cdot \gamma_{i} \cdot E_{i} \cdot \rho_{i}$. 
Observe that the fast-path transaction $T_{i} \in \gamma_{i}$ does not access any t-object that is accessed by any slowpath transaction in the execution fragment $E_{0} \cdots E_{\frac{\left|S_{m}\right|}{2}-1}$. By Lemma 7.8 there exists a t-complete step contention-free execution fragment $E^{\prime}$ that is similar to $E_{0} \cdots E_{\frac{\left|S_{m}\right|}{2}-1}$ such that for all $i \in\left\{0, \ldots, \frac{\left|S_{m}\right|}{2}-\right.$ $1\}, \mathcal{M}$ has an execution of the form $E_{m} \cdot E^{\prime} \cdot \gamma_{i} \cdot \rho_{i}$. By our construction, the enabled event of each fast-path transaction $T_{i} \in \beta_{i}$ in this execution is an access to a distinct metadata base object.

Let $S_{m+1}$ denote the set of all fast-path transactions that participate in the execution fragment $\beta_{0} \cdots \beta_{\frac{\mid\left(S_{m} \mid\right.}{2}-1}$ and $E_{m+1}=E_{m} \cdot E^{\prime}$. Thus, $\left|S_{m+1}\right|$ fast-path transactions, each of which run step contention-free from the same t-quiescent configuration, perform $m+1$ t-reads of distinct t-objects so that at least one distinct metadata base object is accessed within the execution of each t-read operation. This completes the proof.

\subsection{Instrumentation-optimal progressive HyTM}

We prove that the lower bound in Theorem 7.9 is tight by describing an 'instrumentation-optimal" HyTM implementation (Algorithm 7.1) that is opaque, progressive, provides wait-free TM-liveness, uses invisible reads.

Base objects. For every t-object $X_{j}$, our implementation maintains a base object $v_{j} \in \mathbb{D}$ that stores the value of $X_{j}$ and a metadata base object $r_{j}$, which is a lock bit that stores 0 or 1 .

Fast-path transactions. For a fast-path transaction $T_{k}$, the $\operatorname{read}_{k}\left(X_{j}\right)$ implementation first reads $r_{j}$ to check if $X_{j}$ is locked by a concurrent updating transaction. If so, it returns $A_{k}$, else it returns the value of $X_{j}$. Updating fast-path transactions use uninstrumented writes: write $\left(X_{j}, v\right)$ simply stores the cached state of $X_{j}$ along with its value $v$ and if the cache has not been invalidated, updates the shared memory during $\operatorname{try} C_{k}$ by invoking the commit-cache primitive.

Slow-path read-only transactions. Any $\operatorname{read}_{k}\left(X_{j}\right)$ invoked by a slow-path transaction first reads the value of the object from $v_{j}$, checks if $r_{j}$ is set and then performs value-based validation on its entire read set to check if any of them have been modified. If either of these conditions is true, the transaction returns $A_{k}$. Otherwise, it returns the value of $X_{j}$. A read-only transaction simply returns $C_{k}$ during the tryCommit.

Slow-path updating transactions. The write $_{k}(X, v)$ implementation of a slow-path transaction stores $v$ and the current value of $X_{j}$ locally, deferring the actual update in shared memory to tryCommit.

During $\operatorname{try} C_{k}$, an updating slow-path transaction $T_{k}$ attempts to obtain exclusive write access to its entire write set as follows: for every t-object $X_{j} \in W \operatorname{set}\left(T_{k}\right)$, it writes 1 to each base object $r_{j}$ by performing a compare-and-set (cas) primitive that checks if the value of $r_{j}$ is not 1 and, if so, replaces it with 1 . If the cas fails, then $T_{k}$ releases the locks on all objects $X_{\ell}$ it had previously acquired by writing 0 to $r_{\ell}$ and then returns $A_{k}$. Intuitively, if the cas fails, some concurrent transaction is performing a t-write to a t-object in $W \operatorname{set}\left(T_{k}\right)$. If all the locks on the write set were acquired successfully, $T_{k}$ checks if any t-object in $\operatorname{Rset}\left(T_{k}\right)$ is concurrently being updated by another transaction and then performs value-based validation of the read set. If a conflict is detected from the these checks, the transaction is aborted. Finally, $\operatorname{try} C_{k}$ attempts to write the values of the t-objects via cas operations. If any cas on the individual base objects fails, there must be a concurrent fast-path writer, and so $T_{k}$ rolls back the state of the base objects that were updated, releases locks on its write set and returns $A_{k}$. The roll backs are performed with cas operations, skipping any which fail to allow for concurrent fast-path writes to locked locations. Note that if a concurrent read operation of a fast-path transaction $T_{\ell}$ finds an "invalid" value in $v_{j}$ that was written by such transaction $T_{k}$ but has not been rolled back yet, then $T_{\ell}$ either incurs a tracking set abort later because $T_{k}$ has updated $v_{j}$ or finds $r_{j}$ to be 1 . In both cases, the read operation of $T_{\ell}$ aborts.

The implementation uses invisible reads (no nontrivial primitives are applied by reading transactions). Every t-operation returns a matching response within a finite number of its steps. 
\begin{tabular}{l}
\hline Algorithm 7.1 Progressive opaque HyTM implementation that provides uninstrumented writes and \\
invisible reads; code for process $p_{i}$ executing transaction $T_{k}$
\end{tabular}
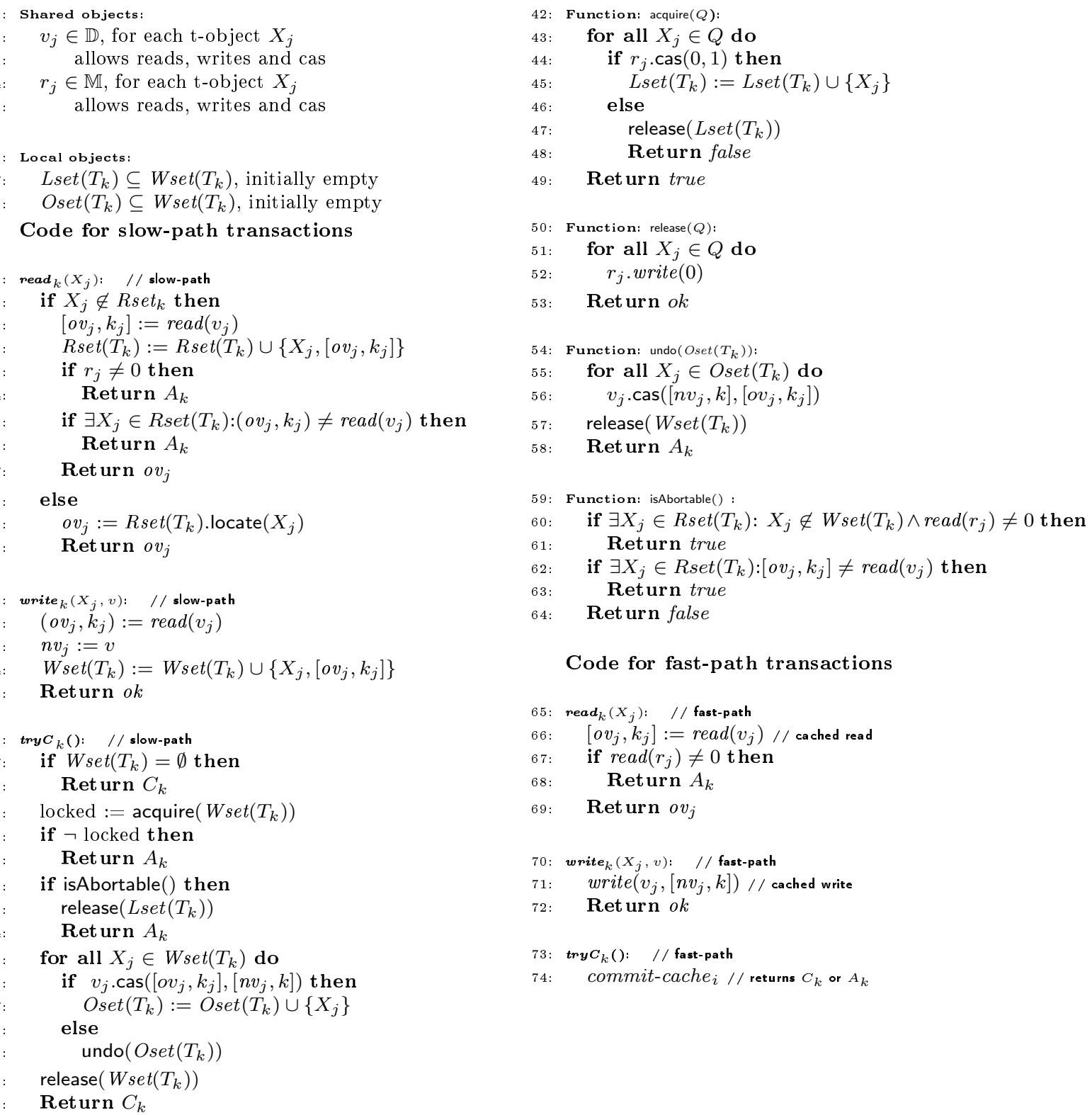

Code for fast-path transactions

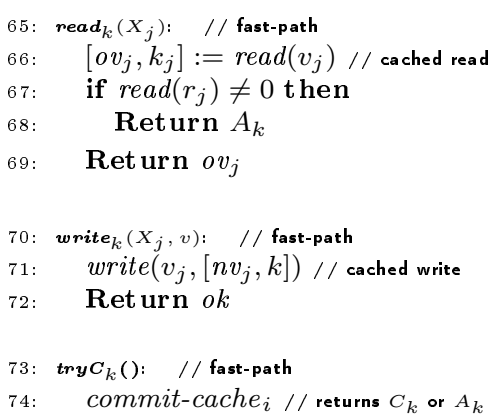

Complexity. Every t-read operation performed by a fast-path transaction accesses a metadata base object once (the lock bit corresponding to the t-object), which is the price to pay for detecting conflicting updating slow-path transactions. Write operations of fast-path transactions are uninstrumented.

Lemma 7.13. Algorithm 7.1 implements an opaque TM.

Proof. Let $E$ by any execution of Algorithm 7.1. Since opacity is a safety property, it is sufficient to prove that every finite execution is opaque [17. Let $<_{E}$ denote a total-order on events in $E$.

Let $H$ denote a subsequence of $E$ constructed by selecting linearization points of t-operations performed in $E$. The linearization point of a t-operation $o p$, denoted as $\ell_{o p}$ is associated with a base object event or an event performed during the execution of op using the following procedure. 
Completions. First, we obtain a completion of $E$ by removing some pending invocations or adding responses to the remaining pending invocations as follows:

- incomplete read r $_{k}$, write $k_{k}$ operation performed by a slow-path transaction $T_{k}$ is removed from $E$; an incomplete $\operatorname{try} C_{k}$ is removed from $E$ if $T_{k}$ has not performed any write to a base object $r_{j}$; $X_{j} \in W \operatorname{set}\left(T_{k}\right)$ in Line 36 , otherwise it is completed by including $C_{k}$ after $E$.

- every incomplete $\operatorname{read}_{k}, \operatorname{try} A_{k}$, write $_{k}$ and $\operatorname{try} C_{k}$ performed by a fast-path transaction $T_{k}$ is removed from $E$.

Linearization points. Now a linearization $H$ of $E$ is obtained by associating linearization points to t-operations in the obtained completion of $E$. For all t-operations performed a slow-path transaction $T_{k}$, linearization points as assigned as follows:

- For every t-read $o p_{k}$ that returns a non- $\mathrm{A}_{k}$ value, $\ell_{o p_{k}}$ is chosen as the event in Line 11 of Algorithm 7.1, else, $\ell_{o p_{k}}$ is chosen as invocation event of $o p_{k}$

- For every $o p_{k}=w_{\text {rite }}$ that returns, $\ell_{o p_{k}}$ is chosen as the invocation event of $o p_{k}$

- For every $o_{k}=\operatorname{try} C_{k}$ that returns $C_{k}$ such that $W \operatorname{set}\left(T_{k}\right) \neq \emptyset, \ell_{o p_{k}}$ is associated with the first write to a base object performed by release when invoked in Line 40 , else if $o p_{k}$ returns $A_{k}, \ell_{o p_{k}}$ is associated with the invocation event of $o p_{k}$

- For every $o p_{k}=\operatorname{try} C_{k}$ that returns $C_{k}$ such that $W \operatorname{set}\left(T_{k}\right)=\emptyset, \ell_{o p_{k}}$ is associated with Line 28

For all t-operations performed a fast-path transaction $T_{k}$, linearization points as assigned as follows:

- For every t-read $o p_{k}$ that returns a non- $\mathrm{A}_{k}$ value, $\ell_{o p_{k}}$ is chosen as the event in Line 66 of Algorithm 7.1, else, $\ell_{o p_{k}}$ is chosen as invocation event of $o p_{k}$

- For every $o p_{k}$ that is a $\operatorname{try} C_{k}, \ell_{o p_{k}}$ is the commit-cache $e_{k}$ primitive invoked by $T_{k}$

- For every $o p_{k}$ that is a write $e_{k}, \ell_{o p_{k}}$ is the event in Line 71 .

$<_{H}$ denotes a total-order on t-operations in the complete sequential history $H$.

Serialization points. The serialization of a transaction $T_{j}$, denoted as $\delta_{T_{j}}$ is associated with the linearization point of a t-operation performed by the transaction.

We obtain a t-complete history $\bar{H}$ from $H$ as follows. A serialization $S$ is obtained by associating serialization points to transactions in $\bar{H}$ as follows: for every transaction $T_{k}$ in $H$ that is complete, but not t-complete, we insert $\operatorname{try} C_{k} \cdot A_{k}$ immediately after the last event of $T_{k}$ in $H$.

- If $T_{k}$ is an updating transaction that commits, then $\delta_{T_{k}}$ is $\ell_{t r y C_{k}}$

- If $T_{k}$ is a read-only or aborted transaction, then $\delta_{T_{k}}$ is assigned to the linearization point of the last t-read that returned a non- $\mathrm{A}_{k}$ value in $T_{k}$

$<_{S}$ denotes a total-order on transactions in the t-sequential history $S$.

Claim 7.14. If $T_{i} \prec_{H} T_{j}$, then $T_{i}<_{S} T_{j}$

Proof. This follows from the fact that for a given transaction, its serialization point is chosen between the first and last event of the transaction implying if $T_{i} \prec_{H} T_{j}$, then $\delta_{T_{i}}<_{E} \delta_{T_{j}}$ implies $T_{i}<_{S} T_{j}$.

Claim 7.15. $S$ is legal. 
Proof. We claim that for every $\operatorname{read}_{j}\left(X_{m}\right) \rightarrow v$, there exists some slow-path transaction $T_{i}$ (or resp. fast-path) that performs write $e_{i}\left(X_{m}, v\right)$ and completes the event in Line 36 (or resp. Line 71) such that $\operatorname{read}_{j}\left(X_{m}\right) \nprec_{H}^{R T}$ write $_{i}\left(X_{m}, v\right)$.

Suppose that $T_{i}$ is a slow-path transaction: since $\operatorname{read}_{j}\left(X_{m}\right)$ returns the response $v$, the event in Line 11 succeeds the event in Line 36 performed by $\operatorname{try} C_{i}$. Since read $\left.d_{m}\right)$ can return a non-abort response only after $T_{i}$ writes 0 to $r_{m}$ in Line 52, $T_{i}$ must be committed in $S$. Consequently, $\ell_{\text {try } C_{i}}<_{E} \ell_{\text {read }_{j}\left(X_{m}\right)}$. Since, for any updating committing transaction $T_{i}, \delta_{T_{i}}=\ell_{t r y C_{i}}$, it follows that $\delta_{T_{i}}<_{E} \delta_{T_{j}}$.

Otherwise if $T_{i}$ is a fast-path transaction, then clearly $T_{i}$ is a committed transaction in $S$. Recall that $\operatorname{read}_{j}\left(X_{m}\right)$ can read $v$ during the event in Line 11 only after $T_{i}$ applies the commit-cache primitive. By the assignment of linearization points, $\ell_{t r y C_{i}}<_{E} \ell_{\text {read }_{j}\left(X_{m}\right)}$ and thus, $\delta_{T_{i}}<_{E} \ell_{\text {read }_{j}\left(X_{m}\right)}$.

Thus, to prove that $S$ is legal, it suffices to show that there does not exist a transaction $T_{k}$ that returns $C_{k}$ in $S$ and performs write $_{k}\left(X_{m}, v^{\prime}\right) ; v^{\prime} \neq v$ such that $T_{i}<_{S} T_{k}<_{S} T_{j}$.

$T_{i}$ and $T_{k}$ are both updating transactions that commit. Thus,

$$
\begin{gathered}
\left(T_{i}<_{S} T_{k}\right) \Longleftrightarrow\left(\delta_{T_{i}}<_{E} \delta_{T_{k}}\right) \\
\left(\delta_{T_{i}}<_{E} \delta_{T_{k}}\right) \Longleftrightarrow\left(\ell_{\text {try } C_{i}}<_{E} \ell_{t r y C_{k}}\right)
\end{gathered}
$$

Since, $T_{j}$ reads the value of $X$ written by $T_{i}$, one of the following is true: $\ell_{\operatorname{try} C_{i}}<_{E} \ell_{\text {try } C_{k}}<_{E} \ell_{\text {read }_{j}\left(X_{m}\right)}$ or $\ell_{t r y C_{i}}<_{E} \ell_{\text {read }_{j}\left(X_{m}\right)}<_{E} \ell_{t r y C_{k}}$.

Suppose that $\ell_{\text {try } C_{i}}<_{E} \ell_{t r y} C_{k}<_{E} \ell_{\text {read }_{j}\left(X_{m}\right)}$.

(Case $I$ :) $T_{i}$ and $T_{k}$ are slow-path transactions.

Thus, $T_{k}$ returns a response from the event in Line 29 before the read of the base object associated with $X_{m}$ by $T_{j}$ in Line 11. Since $T_{i}$ and $T_{k}$ are both committed in $E, T_{k}$ returns true from the event in Line 29 only after $T_{i}$ writes 0 to $r_{m}$ in Line 52 .

If $T_{j}$ is a slow-path transaction, recall that $\operatorname{read}_{j}\left(X_{m}\right)$ checks if $X_{j}$ is locked by a concurrent transaction, then performs read-validation (Line 13 ) before returning a matching response. We claim that $\operatorname{read}_{j}\left(X_{m}\right)$ must return $A_{j}$ in any such execution.

Consider the following possible sequence of events: $T_{k}$ returns true from acquire function invocation, updates the value of $X_{m}$ to shared-memory (Line 36), $T_{j}$ reads the base object $v_{m}$ associated with $X_{m}, T_{k}$ releases $X_{m}$ by writing 0 to $r_{m}$ and finally $T_{j}$ performs the check in Line 13 . But in this case, $\operatorname{read}_{j}\left(X_{m}\right)$ is forced to return the value $v^{\prime}$ written by $T_{m}-$ contradiction to the assumption that $\operatorname{read}_{j}\left(X_{m}\right)$ returns $v$.

Otherwise suppose that $T_{k}$ acquires exclusive access to $X_{m}$ by writing 1 to $r_{m}$ and returns true from the invocation of acquire, updates $v_{m}$ in Line 36, $T_{j}$ reads $v_{m}, T_{j}$ performs the check in Line 13 and finally $T_{k}$ releases $X_{m}$ by writing 0 to $r_{m}$. Again, read ${ }_{j}\left(X_{m}\right)$ must return $A_{j}$ since $T_{j}$ reads that $r_{m}$ is 1 - contradiction.

A similar argument applies to the case that $T_{j}$ is a fast-path transaction. Indeed, since every data base object read by $T_{j}$ is contained in its tracking set, if any concurrent transaction updates any t-object in its read set, $T_{j}$ is aborted immediately by our model(cf. Section 7.2.2).

Thus, $\ell_{\text {try } C_{i}}<_{E} \ell_{\text {read }_{j}(X)}<_{E} \ell_{t r y C_{k}}$.

(Case II:) $T_{i}$ is a slow-path transaction and $T_{k}$ is a fast-path transaction. Thus, $T_{k}$ returns $C_{k}$ before the read of the base object associated with $X_{m}$ by $T_{j}$ in Line 11, but after the response of acquire by $T_{i}$ in Line 29. Since $\operatorname{read}_{j}\left(X_{m}\right)$ reads the value of $X_{m}$ to be $v$ and not $v^{\prime}, T_{i}$ performs the cas to $v_{m}$ in Line 36 after the $T_{k}$ performs the commit-cache primitive (since if otherwise, $T_{k}$ would be aborted in $E)$. But then the cas on $v_{m}$ performed by $T_{i}$ would return false and $T_{i}$ would return $A_{i}$ - contradiction.

(Case III:) $T_{k}$ is a slow-path transaction and $T_{i}$ is a fast-path transaction. This is analogous to the above case. 
(Case $I V:$ ) $T_{i}$ and $T_{k}$ are fast-path transactions. Thus, $T_{k}$ returns $C_{k}$ before the read of the base object associated with $X_{m}$ by $T_{j}$ in Line 11, but before $T_{i}$ returns $C_{i}$ (this follows from Observations 7.1 and 7.2). Consequently, read $\left(X_{m}\right)$ must read the value of $X_{m}$ to be $v^{\prime}$ and return $v^{\prime}$-contradiction.

We now need to prove that $\delta_{T_{j}}$ indeed precedes $\ell_{t r y C_{k}}$ in $E$.

Consider the two possible cases:

- Suppose that $T_{j}$ is a read-only transaction. Then, $\delta_{T_{j}}$ is assigned to the last t-read performed by $T_{j}$ that returns a non- $\mathrm{A}_{j}$ value. If $\operatorname{read}_{j}\left(X_{m}\right)$ is not the last t-read that returned a non- $\mathrm{A}_{j}$ value, then there exists a read ${ }_{j}\left(X^{\prime}\right)$ such that $\ell_{\text {read }}\left(X_{m}\right)<_{E} \ell_{\text {try }_{k}}<_{E} \ell_{\text {read }_{j}\left(X^{\prime}\right)}$. But then this t-read of $X^{\prime}$ must abort by performing the checks in Line 13 or incur a tracking set abort-contradiction.

- Suppose that $T_{j}$ is an updating transaction that commits, then $\delta_{T_{j}}=\ell_{\text {try }} C_{j}$ which implies that $\ell_{\text {read }_{j}(X)}<_{E} \ell_{t r y C_{k}}<_{E} \ell_{t r y C_{j}}$. Then, $T_{j}$ must neccesarily perform the checks in Line 32 and return $A_{j}$ or incur a tracking set abort-contradiction to the assumption that $T_{j}$ is a committed transaction.

The proof follows.

The conjunction of Claims 7.14 and 7.15 establish that Algorithm 7.1 is opaque.

Theorem 7.16. There exists an opaque HyTM implementation that provides uninstrumented writes, invisible reads, progressiveness and wait-free TM-liveness such that in its every execution E, every readonly fast-path transaction $T \in \operatorname{txns}(E)$ accesses $O(|R \operatorname{Ret}(T)|)$ distinct metadata base objects.

\section{Proof. (Opacity) Follows from Lemma 7.13}

(TM-liveness and TM-progress) Since none of the implementations of the t-operations in Algorithm 7.1 contain unbounded loops or waiting statements, Algorithm 7.1 provides wait-free TM-liveness, i.e., every t-operation returns a matching response after taking a finite number of steps.

Consider the cases under which a slow-path transaction $T_{k}$ may be aborted in any execution.

- Suppose that there exists a $\operatorname{read}_{k}\left(X_{j}\right)$ performed by $T_{k}$ that returns $A_{k}$ from Line 13 . Thus, there exists a transaction that has written 1 to $r_{j}$ in Line 44 , but has not yet written 0 to $r_{j}$ in Line 52 or some t-object in $R \operatorname{set}\left(T_{k}\right)$ has been updated since its t-read by $T_{k}$. In both cases, there exists a concurrent transaction performing a t-write to some t-object in $\operatorname{Rset}\left(T_{k}\right)$, thus forcing a read-write conflict.

- Suppose that $t r y C_{k}$ performed by $T_{k}$ that returns $A_{k}$ from Line 30. Thus, there exists a transaction that has written 1 to $r_{j}$ in Line 44 but has not yet written 0 to $r_{j}$ in Line 52 . Thus, $T_{k}$ encounters write-write conflict with another transaction that concurrently attempts to update a t-object in $W \operatorname{set}\left(T_{k}\right)$.

- Suppose that $\operatorname{try} C_{k}$ performed by $T_{k}$ that returns $A_{k}$ from Line 32 . Since $T_{k}$ returns $A_{k}$ from Line 32 for the same reason it returns $A_{k}$ after Line 13 the proof follows.

Consider the cases under which a fast-path transaction $T_{k}$ may be aborted in any execution $E$.

- Suppose that a $\operatorname{read}_{k}\left(X_{m}\right)$ performed by $T_{k}$ returns $A_{k}$ from Line 67. Thus, there exists a concurrent slow-path transaction that is pending in its tryCommit and has written 1 to $r_{m}$, but not released the lock on $X_{m}$ i.e. $T_{k}$ conflicts with another transaction in $E$.

- Suppose that $T_{k}$ returns $A_{k}$ while performing a cached access of some base object $b$ via a trivial (and resp. nontrivial) primitive. Indeed, this is possible only if some concurrent transaction writes (and resp. reads or writes) to $b$. However, two transactions $T_{k}$ and $T_{m}$ may contend on $b$ in $E$ only if there exists $X \in \operatorname{Dset}\left(T_{i}\right) \cap \operatorname{Dset}\left(T_{j}\right)$ and $X \in W \operatorname{set}\left(T_{i}\right) \cup W \operatorname{set}\left(T_{j}\right)$. from Line 30 . The same argument applies for the case when $T_{k}$ returns $A_{k}$ while performing commit-cache $e_{k}$ in $E$. 
Proof. The proof of opacity is almost identical to the analogous proof for Algorithm 7.1 in Lemma 7.13 .

As with Algorithm 7.1, enumerating the cases under which a slow-path transaction $T_{k}$ returns $A_{k}$ proves that Algorithm 7.2 satisfies progressiveness for slow-path transactions. Any fast-path transaction $T_{k}$; $\operatorname{Rset}\left(T_{k}\right) \neq \emptyset$ reads the metadata base object $f a$ and adds it to the process's tracking set (Line 31). If the value of $f a$ is not 0 , indicating that there exists a concurrent slow-path transaction pending in its tryCommit, $T_{k}$ returns $A_{k}$. Thus, the implementation provides sequential TM-progress for fast-path transactions.

Also, in every execution $E$ of $\mathcal{M}$, no fast-path write-only transaction accesses any metadata base object and a fast-path reading transaction accesses the metadata base object $f a$ exactly once, during the first t-read.

\subsection{Related work and Discussion}

HyTM model. Our HyTM model is a natural extension of the model we specified for Software Transactional memory (cf. Chapter 2), and has the advantage of being relatively simple. The term instrumentation was originally used in the context of HyTMs [34, 96, 109 to indicate the overhead a hardware transaction induces in order to detect pending software transactions. The impossibility of designing HyTMs without any code instrumentation was intuitively suggested in [34, we present a formal proof in this paper.

In [22, Attiya and Hillel considered the instrumentation cost of privatization, i.e., allowing transactions to isolate data items by making them private to a process so that no other process is allowed to modify the privatized item. Just as we capture a tradeoff between the cost of hardware instrumentation and the amount of concurrency allowed between hardware and software transactions, [22] captures a tradeoff between the cost of privatization and the number of transactions guaranteed to make progress concurrently in $\ell$-progressive STMs. The model we consider is fundamentally different to [22], in that we model hardware transactions at the level of cache coherence, and do not consider non-transactional accesses, i.e., neither data nor meta-data base objects are private in our HyTM model. The proof techniques we employ are also different.

Uninstrumented HTMs may be viewed as being disjoint-access parallel (DAP) [23, 84]. As such, some of the techniques used in the proof of Theorem 7.4 resemble those used in [23, 58, 62,

We have proved that it is impossible to completely forgo instrumentation in a HyTM even if only sequential TM-progress is required, and that any opaque HyTM implementation providing non-trivial progress either has to pay a linear number of metadata accesses, or will have to allow slow-path transactions to abort fast-path operations. The main motivation for our definition of metadata base objects (Definition 7.1) is given by experiments suggesting that the cost of concurrency detection is a significant bottleneck for many HyTM implementations [99. To precisely characterize the costs incurred by hardware transactions, we made a distinction between the set of memory locations that store the data values of the t-objects and the locations that store the metadata information. To the best of our knowledge, all known HyTM proposals, such as HybridNOrec [34, 109, PhTM [96] and others [36, 86, avoid co-locating the data and metadata within a single base object.

HyTM algorithms. Circa 2005, several papers introduced HyTM implementations [11, 36, 86] that integrated HTMs with variants of DSTM [77. These implementations provide nontrivial concurrency between hardware and software transactions (progressiveness), by imposing instrumentation on hardware transactions: every t-read operation incurs at least one extra access to a metadata base object. Our Theorem 7.9 shows that this overhead is unavoidable. Of note, write operations of these HyTMs are also instrumented, but our Algorithm 7.1 shows that it is not necessary.

Implementations like PhTM [96] and HybridNOrec [34] overcome the per-access instrumentation cost of [36, 86] by realizing that if one is prepared to sacrifice progress, hardware transactions need instrumentation only at the boundaries of transactions to detect pending software transactions. Inspired by 
this observation, our HyTM implementation described in Algorithm 7.2 overcomes the linear per-read instrumentation cost by allowing hardware readers to abort due to a concurrent software writer, but maintains progressiveness for software transactions, unlike [34, 96, 99].

References [67, 109 provide detailed overviews on HyTM designs and implementations. The software component of the HyTM algorithms presented in this paper is inspired by progressive STM implementations [35, 38, 88] and is subject to the lower bounds for progressive STMs established in [22, 60, 62, 88]. 


\section{8 \\ Optimism for boosting concurrency}

The wickedness and the foolishness of no man can avail against the fond optimism of mankind.

James Branch Cabell-The Silver Stallion

\subsection{Overview}

In previous chapters, we were concerned with the inherent complexities of implementing TM. In this chapter, we are concerned with using TM to derive concurrent implementations and raise a fundamental question about the ability of the TM abstraction to transform a sequential implementation to a concurrent one. Specifically, does the optimistic nature of TM give it an inherent advantage in exploiting concurrency that is lacking in pessimistic synchronization techniques like locking? To exploit concurrency, conventional lock-based synchronization pessimistically protects accesses to the shared memory before executing them. Speculative synchronization, achieved using TMs, optimistically executes memory operations with a risk of aborting them in the future. A programmer typically uses these synchronization techniques as "wrappers" to allow every process (or thread) to locally run its sequential code while ensuring that the resulting concurrent execution is globally correct.

Unfortunately, it is difficult for programmers to tell in advance which of the synchronization techniques will establish more concurrency in their resulting programs. In this chapter, we analyze the "amount of concurrency" one can obtain by turning a sequential program into a concurrent one. In particular, we compare the use of optimistic and pessimistic synchronization techniques, whose prime examples are TMs and locks respectively.

To fairly compare concurrency provided by implementations based on various techniques, one has (1) to define what it means for a concurrent program to be correct regardless of the type of synchronization it uses and (2) to define a metric of concurrency.

Correctness. We begin by defining a consistency criterion, namely locally-serializable linearizability. We say that a concurrent implementation of a given sequential data type is locally serializable if it ensures that the local execution of each operation is equivalent to some execution of its sequential implementation. 
This condition is weaker than serializability since it does not require that there exists a single sequential execution that is consistent with all local executions. It is however sufficient to guarantee that optimistic executions do not observe an inconsistent transient state that could lead, for example, to a fatal error like division-by-zero.

Furthermore, the implementation should "make sense" globally, given the sequential type of the data structure we implement. The high-level history of every execution of a concurrent implementation must be linearizable [26, 81] with respect to this sequential type. The combination of local serializability and linearizability gives a correctness criterion that we call LS-linearizability, where LS stands for "locally serializable". We show that LS-linearizability is, as the original linearizability, compositional [79, 81]: a composition of LS-linearizable implementations is also LS-linearizable.

We apply the criterion of LS-linearizability to two broad classes of pessimistic and optimistic synchronization techniques. Pessimistic implementations capture what can be achieved using classic locks; in contrast, optimistic implementations proceed speculatively and fail to return a response to the process in the case of conflicts, e.g., relying on transactional memory.

Measuring concurrency. We characterize the amount of concurrency provided by an LS-linearizable implementation as the set of schedules it accepts. To this end, we define a concurrency metric inspired by the analysis of parallelism in database concurrency control [72, 123. More specifically, we assume an external scheduler that defines which processes execute which steps of the corresponding sequential program in a dynamic and unpredictable fashion. This allows us to define concurrency provided by an implementation as the set of schedules (interleavings of steps of concurrent sequential operations) it accepts (is able to effectively process). Then, the more schedules the implementation would accept, the more concurrent it would be.

We provide a framework to compare the concurrency one can get by choosing a particular synchronization technique for a specific data type. For the first time, we analytically capture the inherent concurrency provided by optimism-based and pessimism-based implementations in exploiting concurrency. We illustrate this using a popular sequential list-based set implementation [79], concurrent implementations of which are our running examples. More precisely, we show that there exist TM-based implementations that, for some workloads, allow for more concurrency than any pessimistic implementation, but we also show that there exist pessimistic implementations that, for other workloads, allow for more concurrency than any TM-based implementation.

Intuitively, an implementation based on transactions may abort an operation based on the way concurrent steps are scheduled, while a pessimistic implementation has to proceed eagerly without knowing about how future steps will be scheduled, sometimes over-conservatively rejecting a potentially acceptable schedule. By contrast, pessimistic implementations designed to exploit the semantics of the data type can supersede the "semantics-oblivious" TM-based implementations. More surprisingly, we demonstrate that combining the benefit of pessimistic implementations, namely their semantics awareness, and the benefit of TMs, namely their optimism, enables implementations that are strictly better-suited for exploiting concurrency than any of them individually. We describe a generic optimistic implementation of the listbased set that is optimal with respect to our concurrency metric: we show that, essentially, it accepts all correct concurrent schedules.

Our results suggest that "relaxed" TM models that are designed with the semantics of the high-level object in mind might be central to exploiting concurrency.

Roadmap of Chapter 8, In Section 8.2 we introduce the class of optimistic and pessimistic concurrent implementations we consider in this chapter. Section 8.3 introduces the definition of locally serializable linearizability and Section 8.4 is devoted to the concurrency analysis of optimistic and pessimistic synchronization techniques in the context of the list-based set. We wrap up with concluding remarks in Section 8.5. 


\subsection{Concurrent implementations}

Objects and implementations. As with Chapter 2, we assume an asynchronous shared-memory system in which a set of $n>1$ processes $p_{1}, \ldots, p_{n}$ communicate by applying operations on shared objects.

An object is an instance of an abstract data type which specifies a set of operations that provide the only means to manipulate the object. Recall that an abstract data type $\tau$ is a tuple $\left(\Phi, \Gamma, Q, q_{0}, \delta\right)$ where $\Phi$ is a set of operations, $\Gamma$ is a set of responses, $Q$ is a set of states, $q_{0} \in Q$ is an initial state and $\delta \subseteq Q \times \Phi \times Q \times \Gamma$ is a transition relation that determines, for each state and each operation, the set of possible resulting states and produced responses. In this chapter, we consider only types that are total, i.e., for every $q \in Q, \pi \in \Phi$, there exist $q^{\prime} \in Q$ and $r \in \Gamma$ such that $\left(q, \pi, q^{\prime}, r\right) \in \delta$. We assume that every type $\tau=\left(\Phi, \Gamma, Q, q_{0}, \delta\right)$ is computable, i.e., there exists a Turing machine that, for each input $(q, \pi), q \in Q, \pi \in \Phi$, computes a pair $\left(q^{\prime}, r\right)$ such that $\left(q, \pi, q^{\prime}, r\right) \in \delta$.

For any type $\tau$, each high-level object $O_{\tau}$ of this type has a sequential implementation. For each operation $\pi \in \Phi, I S$ specifies a deterministic procedure that performs reads and writes on a collection of objects $X_{1}, \ldots, X_{m}$ that encode a state of $O_{\tau}$, and returns a response $r \in \Gamma$.

Sequential list-based set. As a running example, we consider the sorted linked-list based implementation of the type set, commonly referred to as the list-based set [79]. Recall that the set type exports operations $\operatorname{insert}(v), \operatorname{remove}(v)$ and contains $(v)$, with $v \in \mathbb{Z}$. Formally, the set type is defined by the tuple $\left(\Phi, \Gamma, Q, q_{0}, \delta\right)$ where:

$$
\begin{aligned}
& \Phi=\{\operatorname{insert}(v), \text { remove }(v), \text { contains }(v)\} ; v \in \mathbb{Z} \\
& \Gamma=\{\text { true, false }\} \\
& Q \text { is the set of all finite subsets of } \mathbb{Z} ; q_{0}=\emptyset \\
& \delta \text { is defined as follows: }
\end{aligned}
$$

$$
\begin{aligned}
& \text { (1): }(q, \text { contains }(v), q,(v \in q)) \\
& \text { (2): }(q, \operatorname{insert}(v), q \cup\{v\},(v \notin q)) \\
& \text { (3): }(q, \operatorname{remove}(v), q \backslash\{v\},(v \in q))
\end{aligned}
$$

We consider a sequential implementation $L L$ (Algorithm 8.1) of the set type using a sorted linked list where each element (or object) stores an integer value, val, and a pointer to its successor, next, so that elements are sorted in the ascending order of their value.

Every operation invoked with a parameter $v$ traverses the list starting from the head up to the element storing value $v^{\prime} \geq v$. If $v^{\prime}=v$, then contains $(v)$ returns true, remove $(v)$ unlinks the corresponding element and returns true, and $\operatorname{insert}(v)$ returns false. Otherwise, contains $(v)$ and remove $(v)$ return false while insert $(v)$ adds a new element with value $v$ to the list and returns true. The list-based set is denoted by $(L L$, set $)$.

Concurrent implementations. We tackle the problem of turning the sequential implementation $I S$ of type $\tau$ into a concurrent one, shared by $n$ processes. The implementation provides the processes with algorithms for the reads and writes on objects. We refer to the resulting implementation as a concurrent implementation of $(I S, \tau)$. As in Chapter 2, we assume an asynchronous shared-memory system in which the processes communicate by applying primitives on shared base objects $[73$. We place no upper bounds on the number of versions an object may maintain or on the size of this object.

Throughout this chapter, the term operation refers to some high-level operation of the type, while readwrite operations on objects are referred simply as reads and writes.

An implemented read or write may abort by returning a special response $\perp$. In this case, we say that the corresponding high-level operation is aborted. The $\perp$ event is treated both as the response event of the read or write operation and as the response of the corresponding high-level operation. 


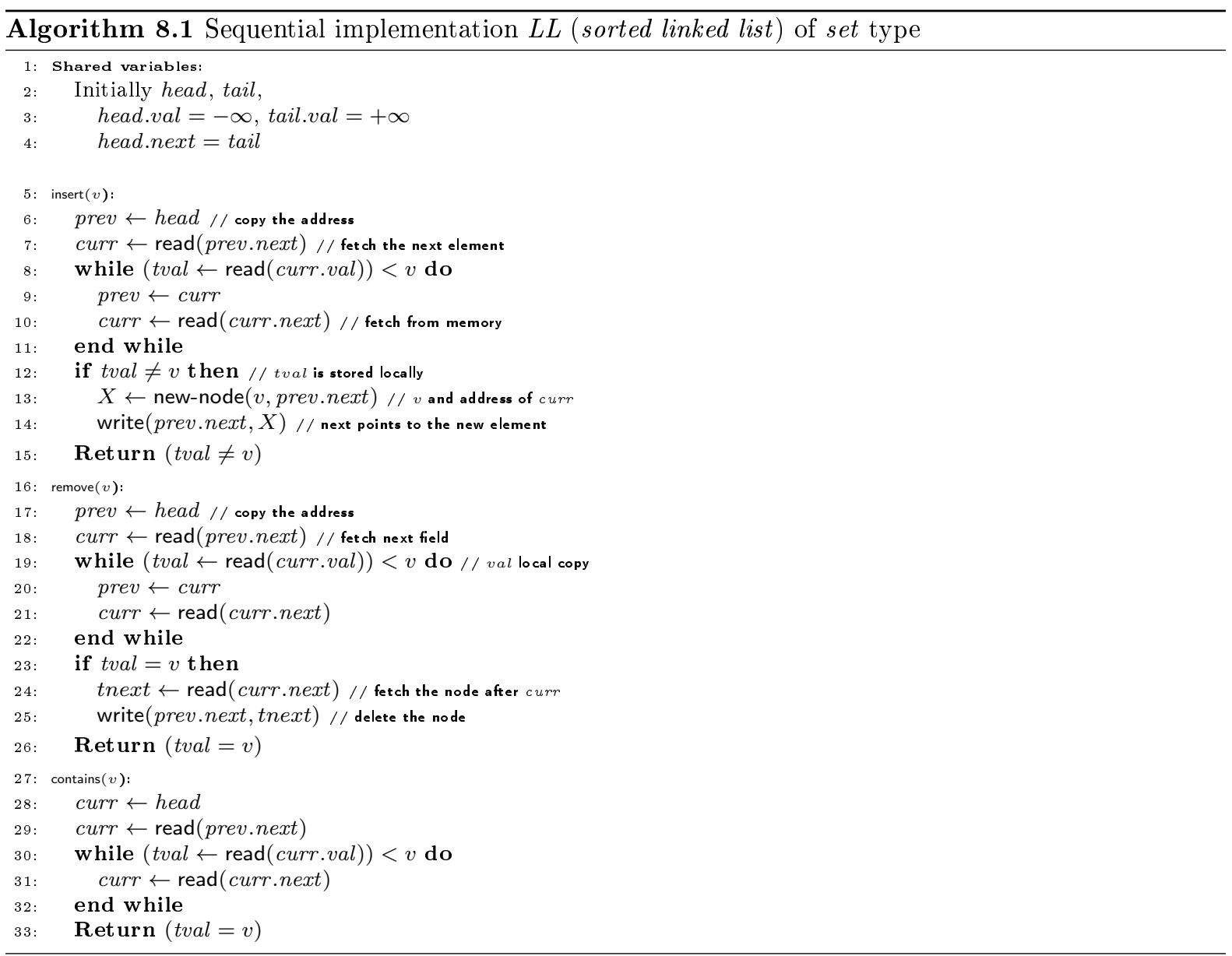

Executions and histories. An execution of a concurrent implementation (of $(I S, \tau)$ ) is a sequence of invocations and responses of high-level operations of type $\tau$, invocations and responses of read and write operations, and primitives applied on base-objects. We assume that executions are well-formed: no process invokes a new read or write, or high-level operation before the previous read or write, or a high-level operation, resp., returns, or takes steps outside its read or write operation's interval.

Let $\alpha \mid p_{i}$ denote the subsequence of an execution $\alpha$ restricted to the events of process $p_{i}$. Executions $\alpha$ and $\alpha^{\prime}$ are equivalent if for every process $p_{i}, \alpha\left|p_{i}=\alpha^{\prime}\right| p_{i}$. An operation $\pi$ precedes another operation $\pi^{\prime}$ in an execution $\alpha$, denoted $\pi \rightarrow_{\alpha} \pi^{\prime}$, if the response of $\pi$ occurs before the invocation of $\pi^{\prime}$. Two operations are concurrent if neither precedes the other. An execution is sequential if it has no concurrent operations. A sequential execution $\alpha$ is legal if for every object $X$, every read of $X$ in $\alpha$ returns the latest written value of $X$. An operation is complete in $\alpha$ if the invocation event is followed by a matching (non- $\perp$ ) response or aborted; otherwise, it is incomplete in $\alpha$. Execution $\alpha$ is complete if every operation is complete in $\alpha$.

The history exported by an execution $\alpha$ is the subsequence of $\alpha$ reduced to the invocations and responses of operations, reads and writes, except for the reads and writes that return $\perp$.

High-level histories and linearizability. A high-level history $\tilde{H}$ of an execution $\alpha$ is the subsequence of $\alpha$ consisting of all invocations and responses of (high-level) operations.

Definition 8.1 (Linearizability). A complete high-level history $\tilde{H}$ is linearizable with respect to an object type $\tau$ if there exists a sequential high-level history $S$ equivalent to $\tilde{H}$ such that $(1) \rightarrow_{\tilde{H}} \subseteq \rightarrow_{S}$ and (2) $S$ is consistent with the sequential specification of type $\tau$.

Now a high-level history $\tilde{H}$ is linearizable if it can be completed (by adding matching responses to a subset of incomplete operations in $\tilde{H}$ and removing the rest) to a linearizable high-level history [26, 81]. 
Obedient implementations. We only consider implementations that satisfy the following condition: Let $\alpha$ be any complete sequential execution of a concurrent implementation $I$. Then in every execution of $I$ of the form $\alpha \cdot \rho_{1} \cdots \rho_{k}$ where each $\rho_{i}(i=1, \ldots, k)$ is the complete execution of a read, every read returns the value written by the last write that does not belong to an aborted operation.

Intuitively, this assumption restricts our scope to "obedient" implementations of reads and writes, where no read value may depend on some future write. In particular, we filter out implementations in which the complete execution of a high-level operation is performed within the first read or write of its sequential implementation.

Pessimistic implementations. Informally, a concurrent implementation is pessimistic if the exported history contains every read-write event that appears in the execution. More precisely, no execution of a pessimistic implementation includes operations that returned $\perp$.

For example, a class of pessimistic implementations are those based on locks. A lock provides shared or exclusive access to an object $X$ through synchronization primitives $\operatorname{lock}^{S}(X)$ (shared mode), $\operatorname{lock}(X)$ (exclusive mode), and unlock $(X)$. When $\operatorname{lock}^{S}(X)(\operatorname{resp}$. lock $(X))$ invoked by a process $p_{i}$ returns, we say that $p_{i}$ holds a lock on $X$ in shared (resp. exclusive) mode. A process releases the object it holds by invoking unlock $(X)$. If no process holds a shared or exclusive lock on $X$, then lock $(X)$ eventually returns; if no process holds an exclusive lock on $X$, then $\operatorname{lock}^{S}(X)$ eventually returns; and if no process holds a

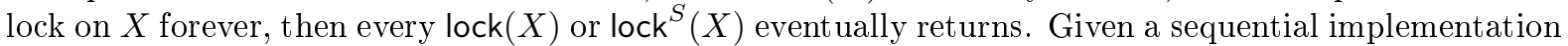
of a data type, a corresponding lock-based concurrent one is derived by inserting the synchronization primitives to provide read-write access to an object.

Optimistic implementations. In contrast with pessimistic ones, optimistic implementations may, under certain conditions, abort an operation: some read or write may return $\perp$, in which case the corresponding operation also returns $\perp$.

Popular classes of optimistic implementations are those based on "lazy synchronization" [69, 79] (with the ability of returning $\perp$ and re-invoking an operation) or transactional memory.

\subsection{Locally serializable linearizability}

We are now ready to define the correctness criterion that we impose on our concurrent implementations.

Let $H$ be a history and let $\pi$ be a high-level operation in $H$. Then $H \mid \pi$ denotes the subsequence of $H$ consisting of the events of $\pi$, except for the last aborted read or write, if any. Let $I S$ be a sequential implementation of an object of type $\tau$ and $\Sigma_{I S}$, the set of histories of $I S$.

Definition 8.2 (LS-linearizability). A history $H$ is locally serializable with respect to IS if for every high-level operation $\pi$ in $H$, there exists $S \in \Sigma_{I S}$ such that $H|\pi=S| \pi$. A history $H$ is LS-linearizable with respect to $(I S, \tau)$ (we also write $H$ is $(I S, \tau)-L S L$ ) if: (1) $H$ is locally serializable with respect to IS and (2) the corresponding high-level history $\tilde{H}$ is linearizable with respect to $\tau$.

Observe that local serializability stipulates that the execution is witnessed sequential by every operation. Two different operations (even when invoked by the same process) are not required to witness mutually consistent sequential executions.

A concurrent implementation $I$ is LS-linearizable with respect to $(I S, \tau)$ (we also write $I$ is $(I S, \tau)$-LSL) if every history exported by $I$ is $(I S, \tau)$-LSL. Throughout this paper, when we refer to a concurrent implementation of $(I S, \tau)$, we assume that it is LS-linearizable with respect to $(I S, \tau)$.

LS-linearizability is compositional. Just as linearizability, LS-linearizability is compositional [79, 81]: a composition of LSL implementations is also LSL. We define the composition of two distinct object types $\tau_{1}$ and $\tau_{2}$ as a type $\tau_{1} \times \tau_{2}=\left(\Phi, \Gamma, Q, q_{0}, \delta\right)$ as follows: $\Phi=\Phi_{1} \cup \Phi_{2}, \Gamma=\Gamma_{1} \cup \Gamma_{2}{ }^{1} Q=Q_{1} \times Q_{2}$,

\footnotetext{
${ }^{1}$ Here we treat each $\tau_{i}$ as a distinct type by adding index $i$ to all elements of $\Phi_{i}, \Gamma_{i}$, and $Q_{i}$.
} 


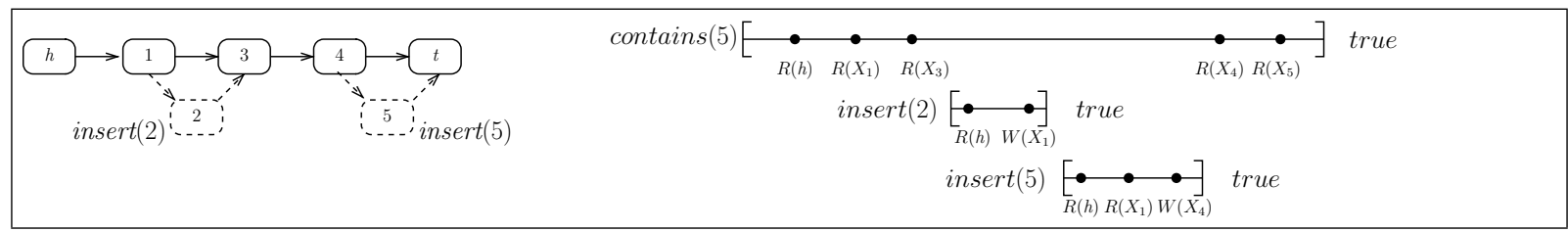

Figure 8.1: A concurrency scenario for a list-based set, initially $\{1,3,4\}$, where value $i$ is stored at node $X_{i}$ : insert(2) and insert(5) can proceed concurrently with contains(5), the history is LS-linearizable but not serializable. (We only depict important read-write events here.)

$q_{0}=\left(q_{01}, q_{02}\right)$, and $\delta \subseteq Q \times \Phi \times Q \times \Gamma$ is such that $\left(\left(q_{1}, q_{2}\right), \pi,\left(q_{1}^{\prime} q_{2}^{\prime}\right), r\right) \in \delta$ if and only if for $i \in\{1,2\}$, if $\pi \in \Phi_{i}$ then $\left(q_{i}, \pi, q_{i}^{\prime}, r\right) \in \delta_{i} \wedge q_{3-i}=q_{3-i}^{\prime}$.

Every sequential implementation $I S$ of an object $O_{1} \times O_{2}$ of a composed type $\tau_{1} \times \tau_{2}$ naturally induces two sequential implementations $I_{S 1}$ and $I_{S 2}$ of objects $O_{1}$ and $O_{2}$, respectively. Now a correctness criterion $\Psi$ is compositional if for every history $H$ on an object composition $O_{1} \times O_{2}$, if $\Psi$ holds for $H \mid O_{i}$ with respect to $I_{S i}$, for $i \in\{1,2\}$, then $\Psi$ holds for $H$ with respect to $I S=I_{S 1} \times I_{S 2}$. Here, $H \mid O_{i}$ denotes the subsequence of $H$ consisting of events on $O_{i}$.

Theorem 8.1. LS-linearizability is compositional.

Proof. Let $H$, a history on $O_{1} \times O_{2}$, be LS-linearizable with respect to $I S$. Let each $H \mid O_{i}, i \in\{1,2\}$, be LS-linearizable with respect to $I_{S i}$. Without loss of generality, we assume that $H$ is complete (if $H$ is incomplete, we consider any completion of it containing LS-linearizable completions of $H \mid O_{1}$ and $\left.H \mid O_{1}\right)$.

Let $\tilde{H}$ be a completion of the high-level history corresponding to $H$ such that $\tilde{H} \mid O_{1}$ and $\tilde{H} \mid O_{2}$ are linearizable with respect to $\tau_{1}$ and $\tau_{2}$, respectively. Since linearizability is compositional [79, 81], $\tilde{H}$ is linearizable with respect to $\tau_{1} \times \tau_{2}$.

Now let, for each operation $\pi, S_{\pi}^{1}$ and $S_{\pi}^{2}$ be any two sequential histories of $I_{S 1}$ and $I_{S 2}$ such that $H|\pi| O_{j}=S_{\pi}^{j} \mid \pi$, for $j \in\{1,2\}$ (since $H \mid O_{1}$ and $H \mid O_{2}$ are LS-linearizable such histories exist). We construct a sequential history $S_{\pi}$ by interleaving events of $S_{\pi}^{1}$ and $S_{\pi}^{2}$ so that $S_{\pi} \mid O_{j}=S_{\pi}^{j}, j \in\{1,2\}$. Since each $S_{\pi}^{j}$ acts on a distinct component $O_{j}$ of $O_{1} \times O_{2}$, every such $S_{\pi}$ is a sequential history of $I S$. We pick one $S_{\pi}$ that respects the local history $H \mid \pi$, which is possible, since $H \mid \pi$ is consistent with both $S_{1} \mid \pi$ and $S_{2} \mid \pi$.

Thus, for each $\pi$, we obtain a history of $I S$ that agrees with $H \mid \pi$. Moreover, the high-level history of $H$ is linearizable. Thus, $H$ is LS-linearizable with respect to $I S$.

LS-linearizability versus other criteria. LS-linearizability is a two-level consistency criterion which makes it suitable to compare concurrent implementations of a sequential data structure, regardless of synchronization techniques they use. It is quite distinct from related criteria designed for database and software transactions, such as serializability [106, 122] and multilevel serializability [121, 122].

For example, serializability [106] prevents sequences of reads and writes from conflicting in a cyclic way, establishing a global order of transactions. Reasoning only at the level of reads and writes may be overly conservative: higher-level operations may commute even if their reads and writes conflict [120]. Consider an execution of a concurrent list-based set depicted in Figure 8.1. We assume here that the set initial state is $\{1,3,4\}$. Operation contains(5) is concurrent, first with operation insert(2) and then with operation insert(5). The history is not serializable: insert(5) sees the effect of insert(2) because $R\left(X_{1}\right)$ by insert(5) returns the value of $X_{1}$ that is updated by insert(2) and thus should be serialized after it. But contains(5) misses element 2 in the linked list, but must see the effect of insert(5) to perform the read of $X_{5}$, i.e., the element created by insert(5). However, this history is LSL since each of the three local histories is consistent with some sequential history of $L L$.

Multilevel serializability [121, 122] was proposed to reason in terms of multiple semantic levels in the same execution. LS-linearizability, being defined for two levels only, does not require a global serialization of low-level operations as 2-level serializability does. LS-linearizability simply requires each 
process to observe a local serialization, which can be different from one process to another. Also, to make it more suitable for concurrency analysis of a concrete data structure, instead of semantic-based commutativity [120, we use the sequential specification of the high-level behavior of the object [81].

Linearizability [26, 81, only accounts for high-level behavior of a data structure, so it does not imply LSlinearizability. For example, Herlihy's universal construction [73] provides a linearizable implementation for any given object type, but does not guarantee that each execution locally appears sequential with respect to any sequential implementation of the type. Local serializability, by itself, does not require any synchronization between processes and can be trivially implemented without communication among the processes. Therefore, the two parts of LS-linearizability indeed complement each other.

\subsection{Pessimistic vs. optimistic synchronization}

In this section, we compare the relative abilities of optimistic and pessimistic synchronization techniques to exploit concurrency in the context of the list-based set.

To characterize the ability of a concurrent implementation to process arbitrary interleavings of sequential code, we introduce the notion of a schedule. Intuitively, a schedule describes the order in which complete high-level operations, and sequential reads and writes are invoked by the user. More precisely, a schedule is an equivalence class of complete histories that agree on the order of invocation and response events of reads, writes and high-level operations, but not necessarily on read values or high-level responses. Thus, a schedule can be treated as a history, where responses of reads and operations are not specified.

We say that an implementation $I$ accepts a schedule $\sigma$ if it exports a history $H$ such that complete $(H)$ exhibits the order of $\sigma$, where complete $(H)$ is the subsequence of $H$ that consists of the events of the complete operations that returned a matching response. We then say that the execution (or history) exports $\sigma$. A schedule $\sigma$ is $(I S, \tau)$-LSL if there exists an $(I S, \tau)$-LSL history that exports $\sigma$.

A synchronization technique is a set of concurrent implementations. We define a specific optimistic synchronization technique and then a specific pessimistic one.

The class $\mathcal{S M}$. Formally, $\mathcal{S M}$ denotes the set of optimistic, safe-strict serializable LSL implementations.

Let $\alpha$ denote the execution of a concurrent implementation and ops $(\alpha)$, the set of operations each of which performs at least one event in $\alpha$. Let $\alpha^{k}$ denote the prefix of $\alpha$ up to the last event of operation $\pi_{k}$. Let $\operatorname{Cseq}(\alpha)$ denote the set of subsequences of $\alpha$ that consist of all the events of operations that are complete in $\alpha$. We say that $\alpha$ is strictly serializable if there exists a legal sequential execution $\alpha^{\prime}$ equivalent to a sequence in $\sigma \in \operatorname{Cseq}(\alpha)$ such that $\rightarrow_{\sigma} \subseteq \rightarrow_{\alpha^{\prime}}$.

We focus on optimistic implementations that are strictly serializable and, in addition, guarantee that every operation (even aborted or incomplete) observes correct (serial) behavior. More precisely, an execution $\alpha$ is safe-strict serializable if (1) $\alpha$ is strictly serializable, and (2) for each operation $\pi_{k}$, there exists a legal sequential execution $\alpha^{\prime}=\pi_{0} \cdots \pi_{i} \cdot \pi_{k}$ and $\sigma \in \operatorname{Cseq}\left(\alpha^{k}\right)$ such that $\left\{\pi_{0}, \cdots, \pi_{i}\right\} \subseteq$ ops $(\sigma)$ and $\forall \pi_{m} \in \operatorname{ops}\left(\alpha^{\prime}\right): \alpha^{\prime}\left|m=\alpha^{k}\right| m$.

Similar to other relaxations of opacity [62] like TMS1 [42] and $V W C$ [83], safe-strict serializable implementations $(\mathcal{S M})$ require that every transaction (even aborted and incomplete) observes "correct" serial behavior. Safe-strict serializability captures nicely both local serializability and linearizability. If we transform a sequential implementation $I S$ of a type $\tau$ into a safe-strict serializable concurrent one, we obtain an LSL implementation of $(I S, \tau)$. Thus, the following lemma is immediate.

Lemma 8.2. Let I be a safe-strict serializable implementation of $(I S, \tau)$. Then, I is LS-linearizable with respect to $(I S, \tau)$. 


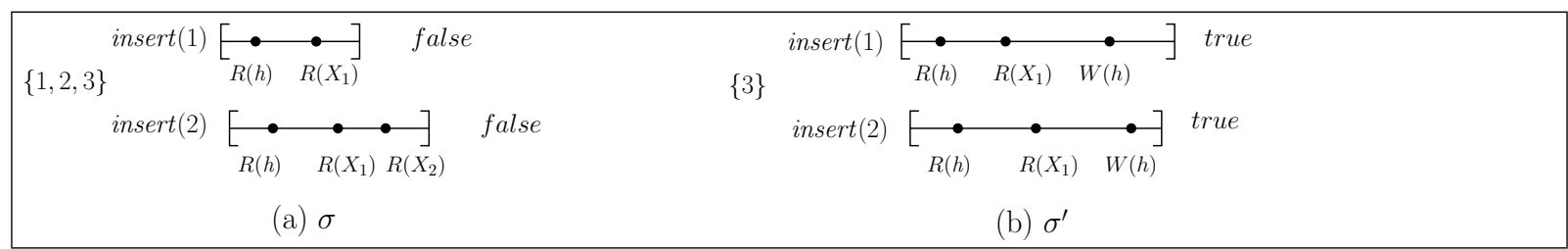

Figure 8.2: (a) a history exporting schedule $\sigma$, with initial state $\{1,2,3\}$, accepted by $I^{L P} \in \mathcal{S M}$; (b) a history exporting a problematic schedule $\sigma^{\prime}$, with initial state $\{3\}$, which should be accepted by any $I \in \mathcal{P}$ if it accepts $\sigma$

Indeed, by running each operation of $I S$ within a transaction of a safe-strict serializable TM, we make sure that completed operations witness the same execution of $I S$, and every operation that returned $\perp$ is consistent with some execution of $I S$ based on previously completed operations.

The class $\mathcal{P}$. This denotes the set of deadlock-free pessimistic LSL implementations: assuming that every process takes enough steps, at least one of the concurrent operations return a matching response [80]. Note that $\mathcal{P}$ includes implementations that are not necessarily safe-strict serializable.

\subsubsection{Concurrency analysis}

We now provide a concurrency analysis of synchronization techniques $\mathcal{S M}$ and $\mathcal{P}$ in the context of the list-based set.

A pessimistic implementation $I^{H} \in \mathcal{P}$ of $(L L$, set). We describe a pessimistic implementation of ( $L L$, set $), I^{H} \in \mathcal{P}$, that accepts non-serializable schedules: each read operation performed by contains acquires the shared lock on the object, reads the next field of the element before releasing the shared lock on the predecessor element in a hand-over-hand manner 28. Update operations (insert and remove) acquire the exclusive lock on the head during read(head) and release it at the end. Every other read operation performed by update operations simply reads the element next field to traverse the list. The write operation performed by an insert or a remove acquires the exclusive lock, writes the value to the element and releases the lock. There is no real concurrency between any two update operations since the process holds the exclusive lock on the head throughout the operation execution. Thus:

Lemma 8.3. $I^{H}$ is deadlock-free and LSL implementation of (LL, set).

On the one hand, the schedule of $\left(L L\right.$, set) depicted in Figure 8.1 which we denote by $\sigma_{0}$, is not serializable and must be rejected by any implementation in $\mathcal{S M}$. However, there exists an execution of $I^{H}$ that exports $\sigma_{0}$ since there is no read-write conflict on any two consecutive elements accessed.

On the other hand, consider the schedule $\sigma$ of $(L L$, set $)$ in Figure 8.2(a). Clearly, $\sigma$ is serializable and is accepted by implementations based on most progressive TMs since there is no read-write conflict. For example, let $I^{L P}$ denote an implementation of $(I S, \tau)$ based on the progressive opaque TM implementation $L P$ in Algorithm 4.1 (Chapter 4). Then, $I^{L P} \in \mathcal{S M}$ and the schedule $\sigma$ is accepted by $I^{L P}$. However, we prove that $\sigma$ is not accepted by any implementation in $\mathcal{P}$. Our proof technique is interesting in its own right: we show that if there exists any implementation in $\mathcal{P}$ that accepts $\sigma$, it must also accept the schedule $\sigma^{\prime}$ depicted in Figure 8.2 (b). In $\sigma^{\prime}$, insert(2) overwrites the write on head performed by insert(1) resulting in a lost update. By deadlock-freedom, there exists an extension of $\sigma^{\prime}$ in which a contains $(1)$ returns false; but this is not a linearizable schedule.

Theorem 8.4. There exists a schedule $\sigma_{0}$ of (LL, set) that is accepted by an implementation in $\mathcal{P} \mathcal{L}$, but not accepted by any implementation $I \in \mathcal{S M}$.

Proof. Let $\sigma_{0}$ be the schedule of $\left(L L\right.$, set) depicted in Figure 8.1. Suppose by contradiction that $\sigma_{0} \in$ $\mathcal{S}(I)$, where $I$ is an implementation of $(L L$, set) based on any safe-strict serializable TM. Thus, there exists an execution $\alpha$ of $I$ that exports $\sigma_{0}$. Now consider two cases: (1) Suppose that the read of $X_{4}$ by 
contains(5) returns the value of $X_{4}$ that is updated by insert(5). Since insert $(2) \rightarrow_{\alpha} \operatorname{insert}(5)$, insert(2) must precede insert(5) in any sequential execution $\alpha^{\prime}$ equivalent to $\alpha$. Also, since contains(5) reads $X_{1}$ prior to its update by insert(2), contains(5) must precede insert(2) in $\alpha^{\prime}$. But then the read of $X_{4}$ is not legal in $\alpha^{\prime}$ - a contradiction since $\alpha$ must be serializable. (2) Suppose that contains(5) reads the initial value of $X_{4}$, i.e., its value prior to the write to $X_{4}$ by insert(5), where $X_{4}$. next points to the tail of the list (according to our sequential implementation $L L$ ). But then, according to $L L$, contains $(5)$ cannot access $X_{5}$ in $\sigma_{0}-$ a contradiction.

Consider the pessimistic implementation $I^{H} \in \mathcal{P}$ : since the contains operation traverses the list using shared hand-over-hand locking, the process $p_{i}$ executing contains(5) can release the lock on element $X_{1}$ prior to the acquisition of the exclusive lock on $X_{1}$ by insert(2). Similarly, $p_{i}$ can acquire the shared lock on $X_{4}$ immediately after the release of the exclusive lock on $X_{4}$ by the process executing insert(5) while still holding the shared lock on element $X_{3}$. Thus, there exists an execution of $I^{H}$ that exports $\sigma_{0}$.

Theorem 8.5. There exists a schedule $\sigma$ of (LL, set) that is accepted by an implementation in $\mathcal{S} \mathcal{M}$, but not accepted by any implementation in $\mathcal{P}$.

Proof. We show first that the schedule $\sigma$ of (LL, set) depicted in Figure 8.2 (a) is not accepted by any implementation in $\mathcal{P}$. Suppose the contrary and let $\sigma$ be exported by an execution $\alpha$. Here $\alpha$ starts with three sequential insert operations with parameters 1,2 , and 3 . The resulting "state" of the set is $\{1,2,3\}$, where value $i \in\{1,2,3\}$ is stored in object $X_{i}$.

Suppose, by contradiction, that some $I \in \mathcal{P}$ accepts $\sigma$. We show that $I$ then accepts the schedule $\sigma^{\prime}$ depicted in Figure 8.2 (b), which starts with a sequential execution of insert(3) storing value 3 in object $X_{1}$.

Let $\alpha^{\prime}$ be any history of $I$ that exports $\sigma^{\prime}$. Recall that we only consider obedient implementations: in $\alpha^{\prime}$ : the read of head by insert(2) in $\sigma^{\prime}$ refers to $X_{1}$ (the next element to be read by insert (2)). In $\alpha$, element $X_{1}$ stores value 1 , i.e., insert(1) can safely return false, while in $\sigma^{\prime}, X_{1}$ stores value 3 , i.e., the next step of insert(1) must be a write to head. Thus, no process can distinguish $\alpha$ and $\alpha^{\prime}$ before the read operations on $X_{1}$ return. Let $\alpha^{\prime \prime}$ be the prefix of $\alpha^{\prime}$ ending with $R\left(X_{1}\right)$ executed by insert $(2)$. Since $I$ is deadlock-free, we have an extension of $\alpha^{\prime \prime}$ in which both insert(1) and insert(2) terminate; we show that this extension violates linearizability. Since $I$ is locally-serializable, to respect our sequential implementation of $(L L$, set), both operations should complete the write to head before returning. Let $\pi_{1}=$ insert(1) be the first operation to write to head in this extended execution. Let $\pi_{2}=\operatorname{insert}(2)$ be the other insert operation. It is clear that $\pi_{1}$ returns true even though $\pi_{2}$ overwrites the update of $\pi_{1}$ on head and also returns true. Recall that implementations in $\mathcal{P}$ are deadlock-free. Thus, we can further extend the execution with a complete contains(1) that will return false (the element inserted to the list by $\pi_{1}$ is lost) - a contradiction since $I$ is linearizable with respect to set. Thus, $\sigma \notin \mathcal{S}(I)$ for any $I \in \mathcal{P}$.

On the other hand, the schedule $\sigma$ is accepted by $I^{L P} \in \mathcal{S M}$, since there is no conflict between the two concurrent update operations.

\subsubsection{Concurrency optimality}

We now combine the benefits of semantics awareness of implementations in $\mathcal{P}$ and the optimism of $\mathcal{S} \mathcal{M}$ to derive a generic optimistic implementation of the list-based set that supersedes every implementation in classes $\mathcal{P}$ and $\mathcal{S M}$ in terms of concurrency. Our implementation, denoted $I^{R M}$ provides processes with algorithms for implementing read and write operations on the elements of the list for each operation of the list-based set (Algorithm 8.2.

Every object (or element) $X_{\ell}$ is specified by the following shared variables: $t$-var $[\ell]$ stores the value $v \in V$ of $X_{\ell}, r[\ell]$ stores a boolean indicating if $X_{\ell}$ is marked for deletion, $L[\ell]$ stores a tuple of the version number of $X_{\ell}$ and a locked flag; the latter indicates whether a concurrent process is performing a write to $X_{\ell}$. 


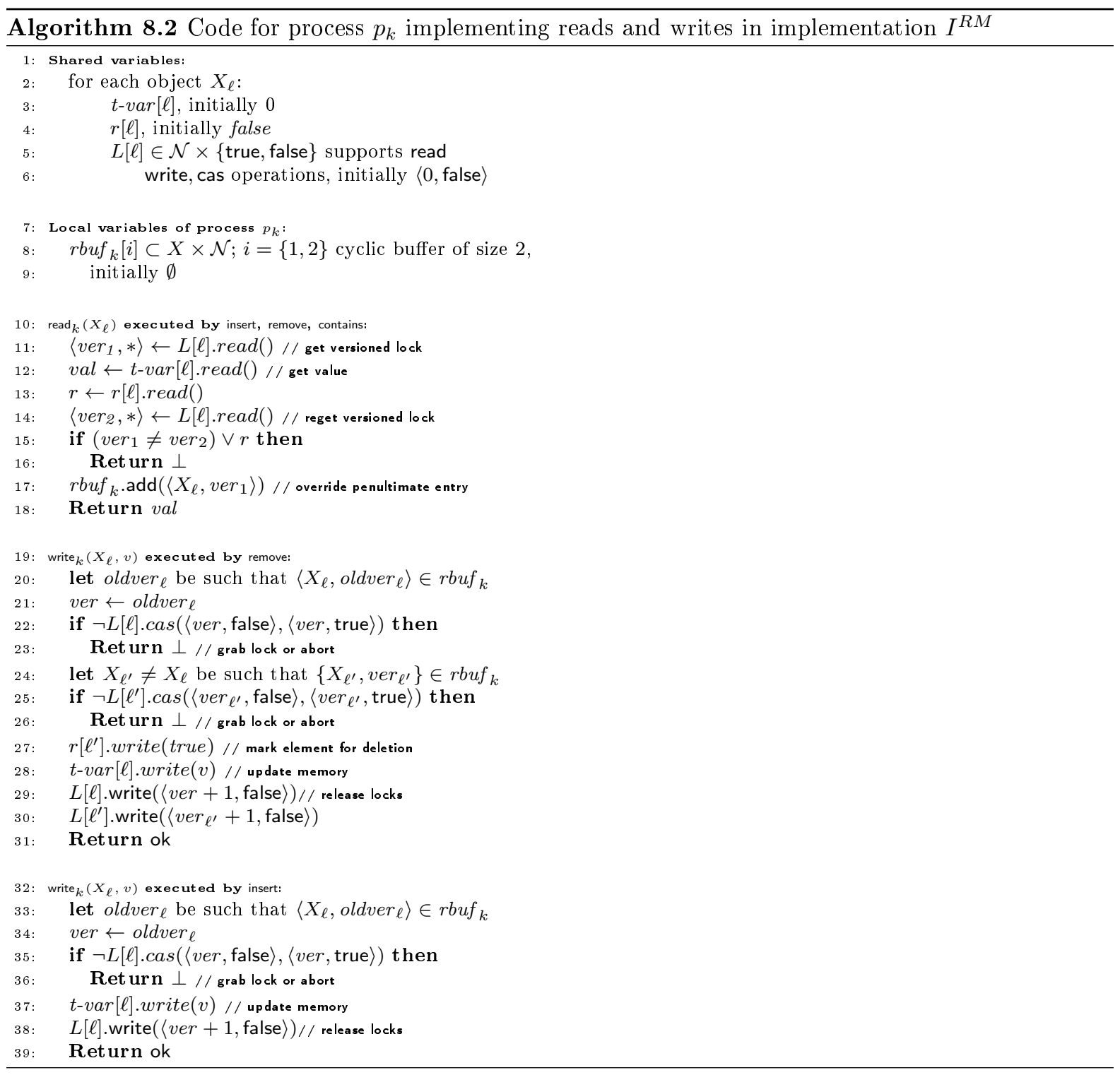

Any operation with input parameter $v$ traverses the list starting from the head element up to the element storing value $v^{\prime} \geq v$ without writing to shared memory. If a read operation on an element conflicts with a write operation to the same element or if the element is marked for deletion, the operation terminates by returning $\perp$. While traversing the list, the process maintains the last two read elements and their version numbers in the local rotating buffer $r b u f$. If none of the read operations performed by $\operatorname{contains}(v)$ return $\perp$ and if $v^{\prime}=v$, then contains $(v)$ returns true; otherwise it returns false. Thus, the contains does not write to shared memory.

To perform write operation to an element as part of an update operation (insert and remove), the process first retrieves the version of the object that belongs to its rotating buffer. It returns $\perp$ if the version has been changed since the previous read of the element or if a concurrent process is executing a write to the same element. Note that, technically, $\perp$ is returned only if prev.next $\rightarrow$ curr. If prev.next $\rightarrow$ curr then we attempt to lock the element with the current version and return $\perp$ if there is a concurrent process executing a write to the same element. But we avoid expanding on this step in our algorithm pseudocode. The write operation performed by the remove operation, additionally checks if the element to be removed from the list is locked by another process; if not, it sets a flag on the element to mark it for deletion. If none of the read or write operations performed during the $\operatorname{insert}(v)$ or remove $(v)$ returned $\perp$, 
appropriate matching responses are returned as prescribed by the sequential implementation $L L$. Any update operation of $I^{R M}$ uses at most two expensive synchronization patterns [16].

Proof of LS-linearizability. Let $\alpha$ be an execution of $I^{R M}$ and $<_{\alpha}$ denote the total-order on events in $\alpha$. For simplicity, we assume that $\alpha$ starts with an artificial sequential execution of an insert operation $\pi_{0}$ that inserts tail and sets head.next $=$ tail. Let $H$ be the history exported by $\alpha$, where all reads and writes are sequential. We construct $H$ by associating a linearization point $\ell_{o p}$ with each non-aborted read or write operation op performed in $\alpha$ as follows:

- if $o p$ is a read, then performed by process $p_{k}, \ell_{o p}$ is the base-object read in line 12

- if $o p$ is a write within an insert operation, $\ell_{o p}$ is the base-object cas in line 22 .

- if $o p$ is a write within a remove operation, $\ell_{o p}$ is the base-object cas in line 35 .

We say that a read of an element $X$ within an operation $\pi$ is valid in $H$ (we also say that $X$ is valid) if there does not exist any remove operation $\pi_{1}$ that deallocates $X$ (removes $X$ from the list) such that $\ell_{\pi_{1} \cdot \operatorname{write}(X)}<_{\alpha} \ell_{\pi \cdot \operatorname{read}(X)}$.

Lemma 8.6. Let $\pi$ be any operation performing $\operatorname{read}(X)$ followed by $\operatorname{read}(Y)$ in $H$. Then (1) there exists an insert operation that sets $X . n e x t=Y$ prior to $\pi \cdot \operatorname{read}(X)$, and (2) $\pi \cdot \operatorname{read}(X)$ and $\pi \cdot \operatorname{read}(Y)$ are valid in $H$.

Proof. Let $\pi$ be any operation in $I^{R M}$ that performs $\operatorname{read}(X)$ followed by $\operatorname{read}(Y)$. If $X$ and $Y$ are head and tail respectively, head.next $=$ tail (by assumption). Since no remove operation deallocates the head or tail, the read of $X$ and $Y$ are valid in $H$.

Now, let $X$ be the head element and suppose that $\pi$ performs $\operatorname{read}(X)$ followed by $\operatorname{read}(Y) ; Y \neq$ tail in $H$. Clearly, if $\pi$ performs a read $(Y)$, there exists an operation $\pi^{\prime}=$ insert that has previously set head.next $=Y$. More specifically, $\pi \cdot \operatorname{read}(X)$ performs the action in line 12 after the write to shared memory by $\pi^{\prime}$ in line 37. By the assignment of linearization points to tx-operations, $\ell_{\pi^{\prime}}<_{\alpha} \ell_{\pi \cdot \operatorname{read}(X)}$. Thus, there exists an insert operation that sets $X$.next $=Y$ prior to $\pi \cdot \operatorname{read}(X)$ in $H$.

For the second claim, we need to prove that the $\operatorname{read}(Y)$ by $\pi$ is valid in $H$. Suppose by contradiction that $Y$ has been deallocated by some $\pi^{\prime \prime}=$ remove operation prior to $\operatorname{read}(Y)$ by $\pi$. By the rules for linearization of read and write operations, the action in line 28 precedes the action in line 12 . However, $\pi$ proceeds to perform the check in line 15 and returns $\perp$ since the flag corresponding to the element $Y$ is previously set by $\pi^{\prime \prime}$. Thus, $H$ does not contain $\pi \cdot \operatorname{read}(Y)$-contradiction.

Inductively, by the above arguments, every non-head read by $\pi$ is performed on an element previously created by an insert operation and is valid in $H$.

Lemma 8.7. $H$ is locally serializable with respect to $L L$.

Proof. By Lemma 8.6, every element $X$ read within an operation $\pi$ is previously created by an insert operation and is valid in $H$. Moreover, if the read operation on $X$ returns $v^{\prime}$, then $X$.next stores a pointer to another valid element that stores an integer value $v^{\prime \prime}>v^{\prime}$. Note that the series of reads performed by $\pi$ terminates as soon as an element storing value $v$ or higher is found. Thus, $\pi$ performs at most $O\left(\left|v-v_{0}\right|\right)$ reads, where $v_{0}$ is the value of the second element read by $\pi$. Now we construct $S^{\pi}$ as a sequence of insert operations, that insert values read by $\pi$, one by one, followed by $\pi$. By construction, $S^{\pi} \in \Sigma_{L L}$.

It is sufficient for us to prove that every finite high-level history $H$ of $I^{R M}$ is linearizable. First, we obtain a completion $\tilde{H}$ of $H$ as follows. The invocation of an incomplete contains operation is discarded. The invocation of an incomplete $\pi=$ insert $\vee$ remove operation that has not returned successfully from the write operation is discarded; otherwise, it is completed with response true.

We obtain a sequential high-level history $\tilde{S}$ equivalent to $\tilde{H}$ by associating a linearization point $\ell_{\pi}$ with each operation $\pi$ as follows. For each $\pi=$ insert $\vee$ remove that returns true in $\tilde{H}, \ell_{\pi}$ is associated with the first write performed by $\pi$ in $H$; otherwise $\ell_{\pi}$ is associated with the last read performed by $\pi$ in $H$. 
For $\pi=$ contains that returns true, $\ell_{\pi}$ is associated with the last read performed in $I^{R M}$; otherwise $\ell_{\pi}$ is associated with the read of head. Since linearization points are chosen within the intervals of operations of $I^{R M}$, for any two operations $\pi_{i}$ and $\pi_{j}$ in $\tilde{H}$, if $\pi_{i} \rightarrow_{\tilde{H}} \pi_{j}$, then $\pi_{i} \rightarrow_{\tilde{S}} \pi_{j}$.

Lemma 8.8. $\tilde{S}$ is consistent with the sequential specification of type set.

Proof. Let $\tilde{S}^{k}$ be the prefix of $\tilde{S}$ consisting of the first $k$ complete operations. We associate each $\tilde{S}^{k}$ with a set $q^{k}$ of objects that were successfully inserted and not subsequently successfully removed in $\tilde{S}^{k}$. We show by induction on $k$ that the sequence of state transitions in $\tilde{S}^{k}$ is consistent with operations' responses in $\tilde{S}^{k}$ with respect to the set type.

The base case $k=1$ is trivial: the tail element containing $+\infty$ is successfully inserted. Suppose that $\tilde{S}^{k}$ is consistent with the set type and let $\pi_{1}$ with argument $v \in \mathbb{Z}$ and response $r_{\pi_{1}}$ be the last operation of $\tilde{S}^{k+1}$. We want to show that $\left(q^{k}, \pi_{1}, q^{k+1}, r_{\pi_{1}}\right)$ is consistent with the set type.

(1) If $\pi_{1}=\operatorname{insert}(v)$ returns true in $\tilde{S}^{k+1}$, there does not exist any other $\pi_{2}=\operatorname{insert}(v)$ that returns true in $\tilde{S}^{k+1}$ such that there does not exist any remove $(v)$ that returns $\operatorname{true} ; \pi_{2} \rightarrow \tilde{S}^{k+1} \operatorname{remove}(v) \rightarrow \tilde{S}^{k+1}$ $\pi_{1}$. Suppose by contradiction that such a $\pi_{1}$ and $\pi_{2}$ exist. Every successful insert $(v)$ operation performs its penultimate read on an element $X$ that stores a value $v^{\prime}<v$ and the last read is performed on an element that stores a value $v^{\prime \prime}>v$. Clearly, $\pi_{1}$ also performs a write on $X$. By construction of $\tilde{S}, \pi_{1}$ is linearized at the release of the cas lock on element $X$. Observe that $\pi_{2}$ must also perform a write to the element $X$ (otherwise one of $\pi_{1}$ or $\pi_{2}$ would return false). By assumption, the write to $X$ in shared-memory by $\pi_{2}$ (line 37) precedes the corresponding write to $X$ in shared-memory by $\pi_{2}$. If $\ell_{\pi_{2}}<_{\alpha} \ell_{\pi_{1} \cdot \operatorname{read}(X)}$, then $\pi_{1}$ cannot return true-a contradiction. Otherwise, if $\ell_{\pi_{1} \cdot \operatorname{read}(X)}<_{\alpha} \ell_{\pi_{2}}$, then $\pi_{1}$ reaches line 22 and return $\perp$. This is because either $\pi_{1}$ attempts to acquire the cas lock on $X$ while it is still held by $\pi_{2}$ or the value of $X$ contained in the rbuf of the process executing $\pi_{1}$ has changed-a contradiction.

If $\pi_{1}=\operatorname{insert}(v)$ returns false in $\tilde{S}^{k+1}$, there exists a $\pi_{2}=\operatorname{insert}(v)$ that returns true in $\tilde{S}^{k+1}$ such that there does not exist any $\pi_{3}=\operatorname{remove}(v)$ that returns true; $\pi_{2} \rightarrow \tilde{S}^{k+1} \pi_{3} \rightarrow_{\tilde{S}^{k+1}} \pi_{1}$. Suppose that such a $\pi_{2}$ does not exist. Thus, $\pi_{1}$ must perform its last read on an element that stores value $v^{\prime \prime}>v$, perform the action in Line 37 and return true - a contradiction.

It is easy to verify that the conjunction of the above two claims prove that $\forall q \in Q ; \forall v \in \mathbb{Z}, \tilde{S}^{k+1}$ satisfies $(q, \operatorname{insert}(v), q \cup\{v\},(v \notin q))$.

(2) If $\pi_{1}=\operatorname{remove}(v)$, similar arguments as applied to insert $(v)$ prove that $\forall q \in Q ; \forall v \in \mathbb{Z}, \tilde{S}^{k+1}$ satisfies $(q$, remove $(v), q \backslash\{v\},(v \in q))$.

(3) If $\pi_{1}=$ contains $(v)$ returns true in $\tilde{S}^{k+1}$, there exists $\pi_{2}=\operatorname{insert}(v)$ that returns true in $\tilde{S}^{k+1}$ such that there does not exist any remove $(v)$ that returns true in $\tilde{S}^{k+1}$ such that $\pi_{2} \rightarrow \tilde{S}^{k+1}$ remove $(v) \rightarrow_{\tilde{S}^{k+1}} \pi_{1}$. The proof of this claim immediately follows from Lemma 8.6.

Now, if $\pi_{1}=$ contains $(v)$ returns false in $\tilde{S}^{k+1}$, there does not exist an $\pi_{2}=\operatorname{insert}(v)$ that returns true such that there does not exist any remove $(v)$ that returns $\operatorname{true} ; \pi_{2} \rightarrow \tilde{S}^{k+1} \operatorname{remove}(v) \rightarrow \tilde{S}^{k+1}$ contains $(v)$. Suppose by contradiction that such a $\pi_{1}$ and $\pi_{2}$ exist. Thus, the action in line 37 by the $\operatorname{insert}(v)$ operation that updates some element, say $X$ precedes the action in line 12 by contains $(v)$ that is associated with its first read (the head). We claim that contains $(v)$ must read the element $X^{\prime}$ newly created by insert $(v)$ and return true-a contradiction to the initial assumption that it returns false. The only case when this can happen is if there exists a remove operation that forces $X^{\prime}$ to be unreachable from head i.e. concurrent to the write to $X$ by insert, there exists a remove that sets $X^{\prime \prime}$.next to $X$.next after the action in line 35 by insert. But this is not possible since the cas on $X$ performed by the remove would return false.

Thus, inductively, the sequence of state transitions in $\tilde{S}$ satisfies the sequential specification of the set type.

Lemmas 8.7 and 8.8 imply: 
Theorem 8.9. $I^{R M}$ is LS-linearizable with respect to $(L L$, set).

Proof of concurrency optimality. Now we show that $I^{R M}$ supersedes, in terms of concurrency, any implementation in classes $\mathcal{P}$ or $\mathcal{S M}$. The proof is based on a more general optimality result, interesting in its own right: any finite schedule rejected by $I^{R M}$ is not observably LS-linearizable (or simply observable). We show that any finite schedule rejected by our algorithm is not observably correct.

A correct schedule $\sigma$ is observably correct if by completing update operations in $\sigma$ and extending, for any $v \in \mathbb{Z}$, the resulting schedule with a complete sequential execution contains $(v)$, applied to the resulting contents of the list, we obtain a correct schedule. Here the contents of the list after a given correct schedule is determined based on the order of its write operations. For each element, we define the resulting state of its next field based on the last write in the schedule. Since in a correct schedule, each new element is first created and then linked to the list, we can reconstruct the state of the list by iteratively traversing it, starting from head.

Intuitively, a schedule is observably correct if it incurs no "lost updates". Consider, for example a schedule (cf. Figure 8.2(b)) in which two operations, insert(1) and insert(2) are applied to the list with state $\{3\}$. The resulting schedule is trivially correct (both operations return true so the schedule can some from a complete linearizable history). However, in the schedule, one of the operations, say insert(1), overwrites the effect of the other one. Thus, if we extend the schedule with a complete execution of contains $(2)$, the only possible response it may give is false which obviously does not produce a linearizable high-level history.

Theorem 8.10 (Optimality). $I^{R M}$ accepts all schedules that are observable with respect to (LL, set).

Proof. We prove that any schedule rejected by $I^{R M}$ is not observable. We go through the cases when a read or write returns $\perp$ (implying the operation fails to return a matching response) and thus the

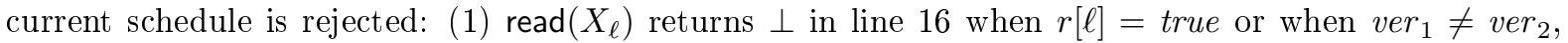
(2) write $\left(X_{\ell}\right)$ performed by remove $(v)$ either returns $\perp$ in line 22 when the cas operation on $L[\ell]$ returns false or returns $\perp$ in line 25 when the cas operation on the element that stores $v$ returns false, and (3) write $\left(X_{\ell}\right)$ performed by insert returns $\perp$ in line 35 when the cas operation on $L[\ell]$ returns false.

Consider the subcase (1a), $r[\ell]$ is set true by a preceding or concurrent write $\left(X_{\ell}\right)$ (line 27). The high-level operation performing this write is a remove that marks the corresponding list element as removed. Since no removed element can be read in a sequential execution of $L L$, the corresponding history is not locally serializable. Alternatively, in subcase (1b), the version of $X_{\ell}$ read previously in line 11 has changed. Thus, an update operation has concurrently performed a write to $X_{\ell}$. However, there exist executions that export such schedules.

In case (2), the write performed by a remove operation returns $\perp$. In subcase (2a), $X_{\ell}$ is currently locked. Thus, a concurrent high-level operation has previously locked $X_{\ell}$ (by successfully performing $L[\ell] \cdot \operatorname{cas}($ ) in line 22 and has not yet released the lock (by writing $\left\langle v e r^{\prime}\right.$, false $\rangle$ to $L[\ell]$ in line 29). In subcase (2b), the current version of $X_{\ell}$ (stored in $L[\ell]$ ) differs from the version of $X_{\ell}$ witnessed by a preceding read. Thus, a concurrent high-level operation completed a write to $X_{\ell}$ after the current high-level operation $\pi$ performed a read of $X_{\ell}$. In both (2a) and (2b), a concurrent high-level updating operation $\pi^{\prime}$ (remove or insert) has written or is about to perform a write to $X_{\ell}$. In subcase $(2 \mathrm{c})$, the cas on the element $X_{\ell^{\prime}}$ (element that stores the value $v$ ) executed by remove $(v)$ returns false (line 25. Recall that by the sequential implementation $L L$, remove $(v)$ performs a read of $X_{\ell^{\prime}}$ prior to the write $\left(X_{\ell}\right)$, where $X_{\ell}$.next refers to $X_{\ell^{\prime}}$. If the cas on $X_{\ell^{\prime}}$ fails, there exists a process that concurrently performed a write to $X_{\ell^{\prime}}$, but after the read of $X_{\ell^{\prime}}$ by remove $(v)$. In all cases, we observe that if we did not abort the write to $X_{\ell}$, then the schedule extended by a complete execution of contains is not LSL.

In case (3), the write performed by an insert operation returns $\perp$. Similar arguments to case (2) prove that any schedule rejected is not observable LSL.

Theorem 8.10 implies that the schedules exported by the histories in Figures 8.1 and 8.2 (a) and that are not accepted by any $I^{\prime} \in \mathcal{S M}$ and any $I \in \mathcal{P}$, respectively, are indeed accepted by $I^{R N}$. But it is easy 
to see that implementations in $\mathcal{S M}$ and $\mathcal{P}$ can only accept observable schedules. As a result, $I^{R M}$ can be shown to strictly supersede any pessimistic or TM-based implementation of the list-based set.

Corollary 8.11. $I^{R M}$ accepts every schedule accepted by any implementation in $\mathcal{P}$ and $\mathcal{S} \mathcal{M}$. Moreover, $I^{R M}$ accepts schedules $\sigma$ and $\sigma^{\prime}$ that are rejected by any implementation in $\mathcal{P}$ and $\mathcal{S} \mathcal{M}$, respectively.

\subsection{Related work and Discussion}

Measuring concurrency. Sets of accepted schedules are commonly used as a metric of concurrency provided by a shared memory implementation. Gramoli et al. 52, defined a concurrency metric, the input acceptance, as the ratio of committed transactions over aborted transactions when TM executes the given schedule. Unlike our metric, input acceptance does not apply to lock-based programs.

For static database transactions, Kung and Papadimitriou [87] use the metric to capture the parallelism of a locking scheme, While acknowledging that the metric is theoretical, they insist that it may have "practical significance as well, if the schedulers in question have relatively small scheduling times as compared with waiting and execution times." Herlihy 72 employed the metric to compare various optimistic and pessimistic synchronization techniques using commutativity of operations constituting high-level transactions. A synchronization technique is implicitly considered in [72] as highly concurrent, namely "optimal", if no other technique accepts more schedules. By contrast, we focus here on a dynamic model where the scheduler cannot use the prior knowledge of all the shared addresses to be accessed. Also, unlike [72, 87, the results in this chapter require all operations, including aborted ones, to observe (locally) consistent states.

Concurrency optimality. This chapter shows that "semantics-oblivious" optimistic TM and "semanticsaware" pessimistic locking are incomparable with respect to exploiting concurrency of the list-based set. Yet, we have shown how to use the benefits of optimism to derive a concurrency optimal implementation that is fine-tuned to the semantics of the list-based set. Intuitively, the ability of an implementation to successfully process interleaving steps of concurrent threads is an appealing property that should be met by performance gains. We believe this to be so.

In work that is not part of the thesis [55], we confirm experimentally that the concurrency optimal optimistic implementation of the list-based set based on $I^{R M}$ outperforms the state-of-the-art implementations of the list-based set, namely, the Lazy linked list [69] and the Harris-Michael linked list [68, 102]. Does the claim also hold for other data structures? We suspect so. For example, similar but more general data structures, such as skip-lists or tree-based dictionaries, may allow for optimizations similar to proposed in this paper. Our results provides some preliminary hints in the quest for the "right" synchronization technique to develop highly concurrent and efficient implementations of data types. 


\section{9}

Concluding remarks

Everything has to come to an end, sometime.

Lyman Frank Baum-The Marvelous Land of $\mathrm{Oz}$

The inclusion of hardware support for transactions in mainstream CPU's [1, 104, 108, suggests that TM is an important concurrency abstraction. However, hardware transactions are not going to be sufficient to support efficient concurrent programming since they may be aborted spuriously; the fast but potentially unreliable hardware transactions must be complemented with slower, but more reliable software transactions. Thus, understanding the inherent cost of both hardware and software transactions is of both theoretical and practical interest.

Below, we briefly recall the outcomes of the thesis and overview the future research directions.

Safety for TMs. We formalized the semantics of a safe TM: every transaction, including aborted and incomplete ones, must observe a view that is consistent with some sequential execution. We introduced the notion of deferred-update semantics which explicitly precludes reading from a transaction that has not yet invoked tryCommit. We believe that our definition is useful to TM practitioners, since it streamlines possible implementations of t-read and tryCommit operations.

Complexity of TMs. The cost of the TM abstraction is parametrized by several properties: safety for transactions, conditions under which transactions must terminate, conditions under which transactions must commit/abort, bound on the number of versions that can be maintained and a multitude of other implementation strategies like disjoint-access parallelism and invisible reads.

At a high-level, the complexity bounds presented in the thesis suggest that providing high degrees of concurrency in software transactional memory (STM) implementations incurs a considerable synchronization cost. As we show, permissive STMs, while providing the best possible concurrency in theory, require a strong synchronization primitive (AWAR) or a memory fence (RAW) per read operation, which may result in excessively slow execution times. Progressive STMs provide only basic concurrency by adapting to data conflicts, but perform considerably better in this respect: we present progressive implementations that incur constant RAW/AWAR complexity.

Since Transactional memory was originally proposed as an alternative to locking, early STMs implementations [51, 77, 98, 114, 117 adopted optimistic concurrency control and guaranteed that a prematurely 
halted transaction cannot not prevent other transactions from committing. However, popular state-ofthe-art STM implementations like TL2 [38] and NOrec [35] are progressive, providing no non-blocking progress guarantees for transactions, but perform empirically better than obstruction-free TMs. Complexity lower and upper bounds presented in the thesis explain this performance gap.

Do our results mean that maximizing the ability of processing multiple transactions in parallel or providing non-blocking progress should not be an important factor in STM design? It would seem so. Should we rather even focus on speculative "single-lock" solutions á la flat combining [70] or "pessimistic" STMs in which transactions never abort [5]? Difficult to say affirmatively, but probably not, since our results suggest progressive STMs incur low complexity overheads as also evidenced by their good empirical performance on most realistic TM workloads [35, 38.

Several questions yet remain open on the complexity of STMs. For instance, the bounds in the thesis were derived for the TM-correctness property of strict serializability and its restrictions. But there has been study of relaxations of strict serializability like snapshot isolation [23, 30. Verifying if the lower bounds presented in the thesis hold under such weak TM-correctness properties and extending the proofs if indeed, presents interesting open questions. The discussion section of Chapters 4,5 and 6 additionally list some unresolved questions closely related to the results in the thesis.

One problem of practical need that is not considered in the thesis concerns the interaction of transactional code with non-transactional code, i.e., the same data item is accessed both transactionally and nontransactionally. It is expected that code executed within a transaction behave as lock-based code within a single "global lock" 101, 113 to avoid memory races. Techniques to ensure the safety of non-transactional accesses have been formulated through the notion of privatization [22, 115]. Devising techniques to ensure privatization for TMs and understanding the cost of enforcing it is an important research direction.

In the thesis, we assumed that a rmw event is an access to a single base object. However, there have been proposals to provide implementations with the ability to invoke $k$-rmw; $k \in \mathbb{N}$ primitives [20, 41] that allow accessing up to $k$ base objects in a single atomic event. For example, the $k$-cas instruction allows to perform $k$ cas instructions atomically on a vector $\left\langle b_{1}, \ldots, b_{k}\right\rangle$ of base objects: it accepts as input a vector $\left\langle\right.$ old $_{1}, \ldots$, old $_{k}$, new $_{1}, \ldots$, new $\left._{k}\right\rangle$ and atomically updates the value of $\left\langle b_{1}, \ldots, b_{k}\right\rangle$ to $\left\langle n e w_{1}, \ldots\right.$, new $\left._{k}\right\rangle$ and returns true iff for all $i \in\{1, \ldots, k\}$, old $i=$ new $_{i}$; otherwise it returns false. However, the ability to access such $k$-rmw primitives does not necessarily simplify the design and improve the performance of non-blocking implementations nor overcome the compositionality issue [20, 41, 79]. Nonetheless, verifying if the lower bounds presented in the thesis hold in this shared memory model is an interesting problem.

HyTMs. We have introduced an analytical model for hybrid transactional memory that captures the notion of cached accesses as performed by hardware transactions. We then derived lower and upper bounds in this model to capture the inherent tradeoff between the degree of concurrency allowed among hardware and software transactions and the instrumentation overhead introduced on the hardware. In a nutshell, our results say that it is impossible to completely forgo instrumentation in a sequential HyTM, and that any opaque HyTM implementation providing non-trivial progress either has to pay a linear number of metadata accesses, or will have to allow slow-path transactions to abort fast-path operations.

Our model of HTMs assumed that the hardware resources were bounded, in the sense that, a hardware transaction may only access a bounded number of data items, exceeding which, it incurs a capacity abort. To overcome the inherent limitations of bounded HTMs, there have been proposals for "unbounded HTMs" that allow transactions to commit even if they exceed the hardware resources [11, 66. The HyTM model from Chapter 7 can be easily extended to accommodate unbounded HTM designs by disregarding capacity aborts.

Some papers have investigated alternatives to providing HTMs with an STM fallback, such as sandboxing [4, 32, or employing hardware-accelerated STM [111, 116], and the use of both direct and cached accesses within the same hardware transaction to reduce instrumentation overhead [86, 109, 110. Another approach proposed reduced hardware transactions [99, where a part of the slow-path is executed 
using a short fast-path transaction, which allows to partially eliminate instrumentation from the hardware fast-path. Modelling and deriving complexity bounds for HyTM proposals outside the HyTM model described in the thesis is an interesting future direction.

Relaxed transactional memory. The concurrency lower bounds derived in Chapter 8 illustrated that a strictly serializable TM, when used as a black-box to transform a sequential implementation of the list-based set to a concurrent one, is not concurrency-optimal. This is due to the fact that TM detects conflicts at the level of transactional reads and writes resulting in false conflicts, in the sense that, the read-write conflict may not affect the correctness of the implemented high-level set type. As we have shown, we can derive a concurrency optimal optimistic (non-strictly serializable) implementation that can process every correct schedule of the list-based set. Indeed, several papers have studied "relaxed" TMs that are fined-tuned to the semantics of the high-level data type [49, 74, 75]. Exploring the complexity of such relaxed TM models represents a very important future research direction. 



\section{List of Figures}

1.1 Transforming a sequential implementation of the list-based set to a TM-based concurrent

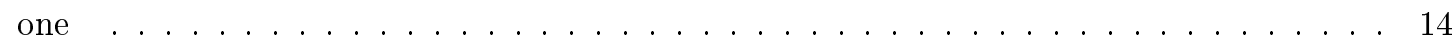

$3.1 \quad$ History $H$ is final-state opaque, while its prefix $H^{\prime}$ is not final-state opaque. . . . . . . . . 35

3.2 An infinite history in which $\operatorname{try} C_{1}$ is incomplete and any two transactions are concurrent. Each finite prefix of the history is du-opaque, but the infinite limit of the ever-extending sequence is not du-opaque. . . . . . . . . . . . . . . . . 37

3.3 A history that is opaque, but not du-opaque. . . . . . . . . . . . . . . . . . . . 41

$3.4 \quad$ A sequential du-opaque history, which is not opaque by the definition of [57]. . . . . . . . 42

3.5 A history that is du-VWC, but not du-opaque. . . . . . . . . . . . . . . . . . . . 44

$3.6 \quad$ A history which is du-VWC but not du-TMS1 . . . . . . . . . . . . . . . . . . 46

3.7 A history which is du-TMS1 but not du-VWC . . . . . . . . . . . . . . . . 46

3.8 A history that is du-opaque, but not TMS2 [42]. . . . . . . . . . . . . . . . . . . . 47

4.1 Executions in the proof of Lemma 4.1 By weak DAP, $T_{\phi}$ cannot distinguish this from the execution in Figure $4.1 \mathrm{a} \mid \ldots \ldots \ldots \ldots \ldots \ldots$

4.2 Execution $E$ of a permissive, opaque TM: $T_{2}$ and $T_{3}$ force $T_{1}$ to perform a RAW/AWAR in each $R_{1}\left(X_{k}\right), 2 \leq k \leq m \ldots \ldots \ldots \ldots \ldots \ldots \ldots$

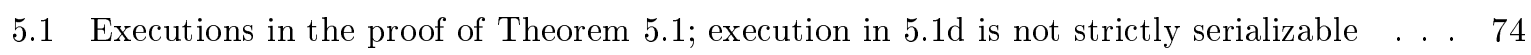

\begin{tabular}{|ll|l|l|l|l|l|l}
5.2 & Executions in the proof of Theorem & 5.4 & execution in & $5.2 \mathrm{e}$ & is not opaque
\end{tabular}$\ldots$. . . . . . . 79

5.3 Complexity gap between blocking and non-blocking TMs . . . . . . . . . . . . . . 87

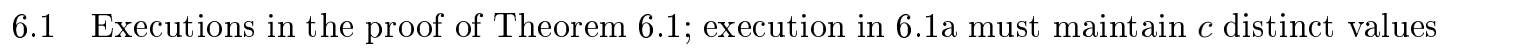

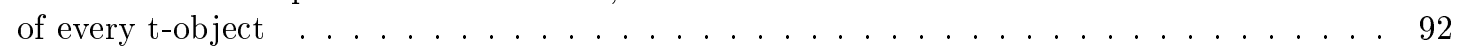

\begin{tabular}{|ll|l|l|l|l|l|}
\hline 6.2 & Executions in the proof of Theorem & 6.2 & execution in & $6.2 \mathrm{c}$ & is not strictly serializable & 94 \\
\hline
\end{tabular}

\begin{tabular}{|ll|l|l|l|l|l|}
\hline 6.3 & Executions in the proof of Theorem & 6.3 & execution in & $6.3 \mathrm{c}$ & is not strictly serializable & . 96 \\
\hline
\end{tabular}

7.1 Tracking set aborts in fast-path transactions; we denote a fast-path (and resp. slow-path)

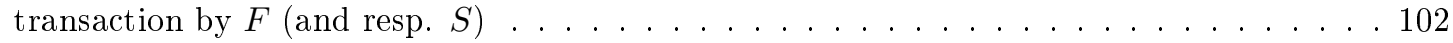

\begin{tabular}{|ll|l|l|l|l|l|l|}
\hline 7.2 & Execution $E$ in Figure & $7.2 \mathrm{a}$ & is indistinguishable to $T_{1}$ from the execution $E^{\prime}$ in Figure & $7.2 \mathrm{~b}$ & 103 \\
\hline
\end{tabular}

\begin{tabular}{|ll|l|l|l|l|}
\hline 7.3 & Executions in the proof of Theorem & 7.4 & execution in & $7.3 \mathrm{~d}$ & is not strictly serializable \\
\hline
\end{tabular} 
8.1 A concurrency scenario for a list-based set, initially $\{1,3,4\}$, where value $i$ is stored at node $X_{i}$ : insert(2) and insert(5) can proceed concurrently with contains(5), the history is LS-linearizable but not serializable. (We only depict important read-write events here.) . . . . . . . . . . . . . 124

8.2 (a) a history exporting schedule $\sigma$, with initial state $\{1,2,3\}$, accepted by $I^{L P} \in \mathcal{S M}$; (b) a history exporting a problematic schedule $\sigma^{\prime}$, with initial state $\{3\}$, which should be accepted by any $I \in \mathcal{P}$ if it accepts $\sigma \ldots \ldots \ldots \ldots \ldots \ldots \ldots \ldots$ 


\section{List of Tables}

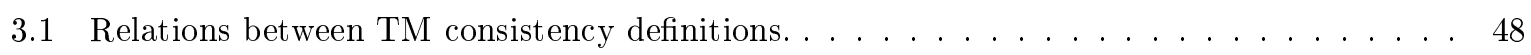

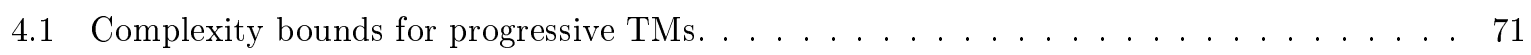

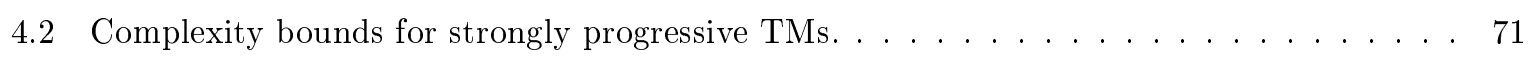





\section{0}

\section{Bibliography}

[1] Advanced Synchronization Facility Proposed Architectural Specification, March 2009. http:// developer.amd.com/wordpress/media/2013/09/45432-ASF_Spec_2.1.pdf

[2] Transactional Memory in GCC, 2012.

[3] S. V. Adve and K. Gharachorloo. Shared memory consistency models: A tutorial. IEEE Computer, 29(12):66-76, 1996.

[4] Y. Afek, A. Levy, and A. Morrison. Software-improved hardware lock elision. In PODC. ACM, 2014.

[5] Y. Afek, A. Matveev, and N. Shavit. Pessimistic software lock-elision. In Proceedings of the 26th International Conference on Distributed Computing, DISC'12, pages 297-311, Berlin, Heidelberg, 2012. Springer-Verlag.

[6] M. K. Aguilera, S. Frølund, V. Hadzilacos, S. L. Horn, and S. Toueg. Abortable and query-abortable objects and their efficient implementation. In PODC, pages $23-32,2007$.

[7] D. Alistarh, P. Eugster, M. Herlihy, A. Matveev, and N. Shavit. Stacktrack: An automated transactional approach to concurrent memory reclamation. In Proceedings of the Ninth European Conference on Computer Systems, EuroSys '14, pages 25:1-25:14, New York, NY, USA, 2014. ACM.

[8] D. Alistarh, J. Kopinsky, P. Kuznetsov, S. Ravi, and N. Shavit. Inherent limitations of hybrid transactional memory. CoRR, abs/1405.5689, 2014.

[9] D. Alistarh, J. Kopinsky, P. Kuznetsov, S. Ravi, and N. Shavit. Inherent limitations of hybrid transactional memory. 6th Workshop on the Theory of Transactional Memory, Paris, France, 2014.

[10] B. Alpern and F. B. Schneider. Defining liveness. Inf. Process. Lett., 21(4):181-185, Oct. 1985.

[11] C. S. Ananian, K. Asanovic, B. C. Kuszmaul, C. E. Leiserson, and S. Lie. Unbounded transactional memory. In Proceedings of the 11th International Symposium on High-Performance Computer Architecture, HPCA '05, pages 316-327, Washington, DC, USA, 2005. IEEE Computer Society.

[12] J. H. Anderson and M. Moir. Universal constructions for multi-object operations. In Proceedings of the Fourteenth Annual ACM Symposium on Principles of Distributed Computing, PODC '95, pages 184-193, New York, NY, USA, 1995. ACM. 
[13] T. E. Anderson. The performance of spin lock alternatives for shared-memory multiprocessors. IEEE Trans. Parallel Distrib. Syst., 1(1):6-16, 1990.

[14] H. Attiya, A. Gotsman, S. Hans, and N. Rinetzky. Safety of live transactions in transactional memory: TMS is necessary and sufficient. In DISC, pages 376-390, 2014.

[15] H. Attiya, R. Guerraoui, D. Hendler, and P. Kuznetsov. The complexity of obstruction-free implementations. J. ACM, 56(4), 2009.

[16] H. Attiya, R. Guerraoui, D. Hendler, P. Kuznetsov, M. Michael, and M. Vechev. Laws of order: Expensive synchronization in concurrent algorithms cannot be eliminated. In POPL, pages 487498, 2011.

[17] H. Attiya, S. Hans, P. Kuznetsov, and S. Ravi. Safety of deferred update in transactional memory. 2013 IEEE 33rd International Conference on Distributed Computing Systems, 0:601-610, 2013.

[18] H. Attiya, S. Hans, P. Kuznetsov, and S. Ravi. Safety of deferred update in transactional memory. CoRR, abs/1301.6297, 2013.

[19] H. Attiya, S. Hans, P. Kuznetsov, and S. Ravi. Safety and deferred update in transactional memory. In R. Guerraoui and P. Romano, editors, Transactional Memory. Foundations, Algorithms, Tools, and Applications, volume 8913 of Lecture Notes in Computer Science, pages 50-71. Springer International Publishing, 2015.

[20] H. Attiya and D. Hendler. Time and space lower bounds for implementations using k-cas. Parallel and Distributed Systems, IEEE Transactions on, 21(2):162 -173, feb. 2010.

[21] H. Attiya, D. Hendler, and P. Woelfel. Tight rmr lower bounds for mutual exclusion and other problems. In Proceedings of the Twenty-seventh ACM Symposium on Principles of Distributed Computing, PODC '08, pages 447-447, New York, NY, USA, 2008. ACM.

[22] H. Attiya and E. Hillel. The cost of privatization in software transactional memory. IEEE Trans. Computers, 62(12):2531-2543, 2013.

[23] H. Attiya, E. Hillel, and A. Milani. Inherent limitations on disjoint-access parallel implementations of transactional memory. Theory of Computing Systems, 49(4):698-719, 2011.

[24] H. Attiya and A. Milani. Transactional scheduling for read-dominated workloads. In Proceedings of the 13th International Conference on Principles of Distributed Systems, OPODIS '09, pages 3-17, Berlin, Heidelberg, 2009. Springer-Verlag.

[25] H. Attiya, G. Ramalingam, and N. Rinetzky. Sequential verification of serializability. In Proceedings of the 37th annual ACM SIGPLAN-SIGACT symposium on Principles of programming languages, pages 31-42, 2010.

[26] H. Attiya and J. Welch. Distributed Computing. Fundamentals, Simulations, and Advanced Topics. John Wiley \& Sons, 2004.

[27] G. Barnes. A method for implementing lock-free shared-data structures. In Proceedings of the Fifth Annual ACM Symposium on Parallel Algorithms and Architectures, SPAA '93, pages 261-270, New York, NY, USA, 1993. ACM.

[28] R. Bayer and M. Schkolnick. Concurrency of operations on B-trees. In Readings in database systems, pages 129-139. Morgan Kaufmann Publishers Inc., 1988.

[29] E. Bruno. What Is Priority Inversion (And How Do You Control It)? 2011.

[30] V. Bushkov, D. Dziuma, P. Fatourou, and R. Guerraoui. The pcl theorem: Transactions cannot be parallel, consistent and live. In SPAA, pages 178-187, 2014.

[31] V. Bushkov, R. Guerraoui, and M. Kapalka. On the liveness of transactional memory. In Proceedings of the 2012 ACM Symposium on Principles of Distributed Computing, PODC '12, pages 9-18, New York, NY, USA, 2012. ACM. 
[32] I. Calciu, T. Shpeisman, G. Pokam, and M. Herlihy. Improved single global lock fallback for best-effort hardware transactional memory. In Transact 2014 Workshop. ACM, 2014.

[33] T. Crain, D. Imbs, and M. Raynal. Read invisibility, virtual world consistency and permissiveness are compatible. Research Report, ASAP - INRIA - IRISA - CNRS : UMR6074 - INRIA - Institut National des Sciences Appliquées de Rennes - Université de Rennes I, 112010.

[34] L. Dalessandro, F. Carouge, S. White, Y. Lev, M. Moir, M. L. Scott, and M. F. Spear. Hybrid NOrec: a case study in the effectiveness of best effort hardware transactional memory. In R. Gupta and T. C. Mowry, editors, ASPLOS, pages 39-52. ACM, 2011.

[35] L. Dalessandro, M. F. Spear, and M. L. Scott. Norec: Streamlining stm by abolishing ownership records. SIGPLAN Not., 45(5):67-78, Jan. 2010.

[36] P. Damron, A. Fedorova, Y. Lev, V. Luchangco, M. Moir, and D. Nussbaum. Hybrid transactional memory. SIGPLAN Not., 41(11):336-346, Oct. 2006.

[37] D. Dice, Y. Lev, M. Moir, and D. Nussbaum. Early experience with a commercial hardware transactional memory implementation. In Proceedings of the 14 th International Conference on Architectural Support for Programming Languages and Operating Systems, ASPLOS XIV, pages 157-168, New York, NY, USA, 2009. ACM.

[38] D. Dice, O. Shalev, and N. Shavit. Transactional locking ii. In Proceedings of the 20th International Conference on Distributed Computing, DISC'06, pages 194-208, Berlin, Heidelberg, 2006. SpringerVerlag.

[39] D. Dice and N. Shavit. What really makes transactions fast? In Transact, 2006.

[40] E. W. Dijkstra. Solution of a problem in concurrent programming control. Commun. ACM, 8(9):569-, Sept. 1965.

[41] S. Doherty, D. L. Detlefs, L. Groves, C. H. Flood, V. Luchangco, P. A. Martin, M. Moir, N. Shavit, and G. L. Steele, Jr. Dcas is not a silver bullet for nonblocking algorithm design. In Proceedings of the Sixteenth Annual ACM Symposium on Parallelism in Algorithms and Architectures, SPAA '04, pages 216-224, New York, NY, USA, 2004. ACM.

[42] S. Doherty, L. Groves, V. Luchangco, and M. Moir. Towards formally specifying and verifying transactional memory. Formal Asp. Comput., 25(5):769-799, 2013.

[43] A. Dragojević, M. Herlihy, Y. Lev, and M. Moir. On the power of hardware transactional memory to simplify memory management. In Proceedings of the 30th Annual ACM SIGACT-SIGOPS Symposium on Principles of Distributed Computing, PODC '11, pages 99-108, New York, NY, USA, 2011. ACM.

[44] F. Ellen, P. Fatourou, E. Kosmas, A. Milani, and C. Travers. Universal constructions that ensure disjoint-access parallelism and wait-freedom. In PODC, pages 115-124, 2012.

[45] F. Ellen, D. Hendler, and N. Shavit. On the inherent sequentiality of concurrent objects. SIAM J. Comput., 41(3):519-536, 2012.

[46] R. Ennals. The lightweight transaction library. http://sourceforge.net/projects/libltx/files/.

[47] R. Ennals. Software transactional memory should not be obstruction-free. 2005.

[48] P. Fatourou and N. D. Kallimanis. A highly-efficient wait-free universal construction. In Proceedings of the Twenty-third Annual ACM Symposium on Parallelism in Algorithms and Architectures, SPAA '11, pages 325-334, New York, NY, USA, 2011. ACM.

[49] P. Felber, V. Gramoli, and R. Guerraoui. Elastic transactions. In DISC, pages 93-107, 2009.

[50] F. Fich, D. Hendler, and N. Shavit. On the inherent weakness of conditional synchronization primitives. In Proceedings of the Twenty-third Annual ACM Symposium on Principles of Distributed Computing, PODC '04, pages 80-87, New York, NY, USA, 2004. ACM. 
[51] K. Fraser. Practical lock-freedom. Technical report, Cambridge University Computer Laborotory, 2003.

[52] V. Gramoli, D. Harmanci, and P. Felber. On the input acceptance of transactional memory. Parallel Processing Letters, 20(1):31-50, 2010.

[53] V. Gramoli, P. Kuznetsov, and S. Ravi. From sequential to concurrent: correctness and relative efficiency (ba). In Principles of Distributed Computing (PODC), pages 241-242, 2012.

[54] V. Gramoli, P. Kuznetsov, and S. Ravi. Optimism for boosting concurrency. CoRR, abs/1203.4751, 2012 .

[55] V. Gramoli, P. Kuznetsov, S. Ravi, and D. Shang. A concurrency-optimal list-based set. CoRR, abs/1502.01633, 2015.

[56] J. Gray and A. Reuter. Transaction Processing: Concepts and Techniques. Morgan Kaufmann Publishers Inc., San Francisco, CA, USA, 1st edition, 1992.

[57] R. Guerraoui, T. A. Henzinger, and V. Singh. Permissiveness in transactional memories. In DISC, pages $305-319,2008$.

[58] R. Guerraoui and M. Kapalka. On obstruction-free transactions. In Proceedings of the twentieth annual symposium on Parallelism in algorithms and architectures, SPAA '08, pages 304-313, New York, NY, USA, 2008. ACM.

[59] R. Guerraoui and M. Kapalka. On the correctness of transactional memory. In Proceedings of the 13th ACM SIGPLAN Symposium on Principles and Practice of Parallel Programming, PPoPP '08, pages 175-184, New York, NY, USA, 2008. ACM.

[60] R. Guerraoui and M. Kapalka. The semantics of progress in lock-based transactional memory. SIGPLAN Not., 44(1):404-415, Jan. 2009.

[61] R. Guerraoui and M. Kapalka. Transactional memory: Glimmer of a theory. In Proceedings of the 21st International Conference on Computer Aided Verification, CAV '09, pages 1-15, Berlin, Heidelberg, 2009. Springer-Verlag.

[62] R. Guerraoui and M. Kapalka. Principles of Transactional Memory, Synthesis Lectures on Distributed Computing Theory. Morgan and Claypool, 2010.

[63] R. Guerraoui, M. Kapalka, and J. Vitek. Stmbench7: A benchmark for software transactional memory. SIGOPS Oper. Syst. Rev., 41(3):315-324, Mar. 2007.

[64] R. Guerraoui and E. Ruppert. Linearizability is not always a safety property. In NETYS, pages $57-69,2014$.

[65] P. K. Hagit Attiya, Sandeep Hans and S. Ravi. What is safe in transactional memory. 4th Workshop on the Theory of Transactional Memory, Madeira, Portugal, 2012.

[66] L. Hammond, V. Wong, M. Chen, B. D. Carlstrom, J. D. Davis, B. Hertzberg, M. K. Prabhu, H. Wijaya, C. Kozyrakis, and K. Olukotun. Transactional memory coherence and consistency. SIGARCH Comput. Archit. News, 32(2):102-, Mar. 2004.

[67] T. Harris, J. R. Larus, and R. Rajwar. Transactional Memory, 2nd edition. Synthesis Lectures on Computer Architecture. Morgan \& Claypool Publishers, 2010.

[68] T. L. Harris. A pragmatic implementation of non-blocking linked-lists. In DISC, pages 300-314, 2001.

[69] S. Heller, M. Herlihy, V. Luchangco, M. Moir, W. N. Scherer, and N. Shavit. A lazy concurrent list-based set algorithm. In OPODIS, pages 3-16, 2006.

[70] D. Hendler, I. Incze, N. Shavit, and M. Tzafrir. Flat combining and the synchronization-parallelism tradeoff. In $S P A A$, pages $355-364,2010$. 
[71] J. L. Hennessy and D. A. Patterson. Computer Architecture: A Quantitative Approach. Morgan Kaufmann Publishers Inc., San Francisco, CA, USA, 3 edition, 2003.

[72] M. Herlihy. Apologizing versus asking permission: optimistic concurrency control for abstract data types. ACM Trans. Database Syst., 15(1):96-124, 1990.

[73] M. Herlihy. Wait-free synchronization. ACM Trans. Prog. Lang. Syst., 13(1):123-149, 1991.

[74] M. Herlihy and E. Koskinen. Transactional boosting: A methodology for highly-concurrent transactional objects. In PPoPP, New York, NY, USA, 2008. ACM.

[75] M. Herlihy and E. Koskinen. Composable transactional objects: A position paper. In Z. Shao, editor, Programming Languages and Systems, volume 8410 of Lecture Notes in Computer Science, pages 1-7. Springer Berlin Heidelberg, 2014.

[76] M. Herlihy, V. Luchangco, and M. Moir. Obstruction-free synchronization: Double-ended queues as an example. In ICDCS, pages 522-529, 2003.

[77] M. Herlihy, V. Luchangco, M. Moir, and W. N. Scherer, III. Software transactional memory for dynamic-sized data structures. In Proceedings of the Twenty-second Annual Symposium on Principles of Distributed Computing, PODC '03, pages 92-101, New York, NY, USA, 2003. ACM.

[78] M. Herlihy and J. E. B. Moss. Transactional memory: architectural support for lock-free data structures. In ISCA, pages 289-300, 1993.

[79] M. Herlihy and N. Shavit. The art of multiprocessor programming. Morgan Kaufmann, 2008.

[80] M. Herlihy and N. Shavit. On the nature of progress. In OPODIS, pages 313-328, 2011.

[81] M. Herlihy and J. M. Wing. Linearizability: A correctness condition for concurrent objects. $A C M$ Trans. Program. Lang. Syst., 12(3):463-492, 1990.

[82] L. Hyonho. Local-spin mutual exclusion algorithms on the DSM model using fetch-and-store objects, 2003.

[83] D. Imbs and M. Raynal. Virtual world consistency: A condition for STM systems (with a versatile protocol with invisible read operations). Theor. Comput. Sci., 444, July 2012.

[84] A. Israeli and L. Rappoport. Disjoint-access-parallel implementations of strong shared memory primitives. In $P O D C$, pages 151-160, 1994.

[85] D. König. Theorie der Endlichen und Unendlichen Graphen: Kombinatorische Topologie der Streckenkomplexe. Akad. Verlag. 1936.

[86] S. Kumar, M. Chu, C. J. Hughes, P. Kundu, and A. Nguyen. Hybrid transactional memory. In Proceedings of the Eleventh ACM SIGPLAN Symposium on Principles and Practice of Parallel Programming, PPoPP '06, pages 209-220, New York, NY, USA, 2006. ACM.

[87] H. T. Kung and C. H. Papadimitriou. An optimality theory of concurrency control for databases. In SIGMOD, pages 116-126, 1979.

[88] P. Kuznetsov and S. Ravi. On the cost of concurrency in transactional memory. In OPODIS, pages 112-127, 2011.

[89] P. Kuznetsov and S. Ravi. On the cost of concurrency in transactional memory. CoRR, abs/1103.1302, 2011.

[90] P. Kuznetsov and S. Ravi. On partial wait-freedom in transactional memory. CoRR, abs/1407.6876, 2014.

[91] P. Kuznetsov and S. Ravi. On partial wait-freedom in transactional memory. In Proceedings of the 2015 International Conference on Distributed Computing and Networking, ICDCN 2015, Goa, India, January 4-7, 2015, page 10, 2015.

[92] P. Kuznetsov and S. Ravi. Progressive transactional memory in time and space. CoRR, abs/1502.04908, 2015. 
[93] P. Kuznetsov and S. Ravi. Progressive transactional memory in time and space. CoRR, abs/1502.04908, 2015. To appear in 13th International Conference on Parallel Computing Technologies, Russia.

[94] P. Kuznetsov and S. Ravi. Why transactional memory should not be obstruction-free. CoRR, abs/1502.02725, 2015.

[95] M. Lesani, V. Luchangco, and M. Moir. Putting opacity in its place. In WTTM, 2012.

[96] Y. Lev, M. Moir, and D. Nussbaum. Phtm: Phased transactional memory. In In Workshop on Transactional Computing (Transact), 200\%. research.sun.com/scalable/pubs/ TRANSACT2007PhTM.pdf.

[97] N. A. Lynch. Distributed Algorithms. Morgan Kaufmann, 1996.

[98] V. J. Marathe, W. N. S. Iii, and M. L. Scott. Adaptive software transactional memory. In In Proc. of the 19th Intl. Symp. on Distributed Computing, pages 354-368, 2005.

[99] A. Matveev and N. Shavit. Reduced hardware transactions: a new approach to hybrid transactional memory. In Proceedings of the 25th ACM symposium on Parallelism in algorithms and architectures, pages 11-22. ACM, 2013.

[100] P. E. McKenney. Memory barriers: a hardware view for software hackers. Linux Technology Center, IBM Beaverton, June 2010.

[101] V. Menon, S. Balensiefer, T. Shpeisman, A.-R. Adl-Tabatabai, R. L. Hudson, B. Saha, and A. Welc. Single global lock semantics in a weakly atomic stm. SIGPLAN Not., 43(5):15-26, May 2008.

[102] M. M. Michael. High performance dynamic lock-free hash tables and list-based sets. In $S P A A$, pages $73-82,2002$.

[103] M. M. Michael and M. L. Scott. Simple, fast, and practical non-blocking and blocking concurrent queue algorithms. In $P O D C$, pages 267-275, 1996.

[104] M. Ohmacht. Memory Speculation of the Blue Gene/Q Compute Chip, 2011. http://wands.cse. lehigh.edu/IBM_BQC_PACT2011.ppt.

[105] S. S. Owicki and L. Lamport. Proving liveness properties of concurrent programs. ACM Trans. Program. Lang. Syst., 4(3):455-495, 1982.

[106] C. H. Papadimitriou. The serializability of concurrent database updates. J. ACM, 26:631-653, 1979.

[107] D. Perelman, R. Fan, and I. Keidar. On maintaining multiple versions in STM. In PODC, pages 16-25, 2010.

[108] J. Reinders. Transactional Synchronization in Haswell, 2012. http://software.intel.com/ en-us/blogs/2012/02/07/transactional-synchronization-in-haswell/

[109] T. Riegel. Software Transactional Memory Building Blocks. 2013.

[110] T. Riegel, P. Marlier, M. Nowack, P. Felber, and C. Fetzer. Optimizing hybrid transactional memory: The importance of nonspeculative operations. In Proceedings of the 23rd ACM Symposium on Parallelism in Algorithms and Architectures, pages 53-64. ACM, 2011.

[111] B. Saha, A.-R. Adl-Tabatabai, and Q. Jacobson. Architectural support for software transactional memory. In Proceedings of the 39th Annual IEEE/ACM International Symposium on Microarchitecture, MICRO 39, pages 185-196, Washington, DC, USA, 2006. IEEE Computer Society.

[112] W. N. Scherer, III and M. L. Scott. Advanced contention management for dynamic software transactional memory. In Proceedings of the Twenty-fourth Annual ACM Symposium on Principles of Distributed Computing, PODC '05, pages 240-248, New York, NY, USA, 2005. ACM.

[113] M. L. Scott. Shared-memory Synchronization, Synthesis Lectures on Distributed Computing Theory. Morgan and Claypool, 2013. 
[114] N. Shavit and D. Touitou. Software transactional memory. In PODC, pages 204-213, 1995.

[115] M. F. Spear, V. J. Marathe, L. Dalessandro, and M. L. Scott. Privatization techniques for software transactional memory. In Proceedings of the Twenty-sixth Annual ACM Symposium on Principles of Distributed Computing, PODC '07, pages 338-339, New York, NY, USA, 2007. ACM.

[116] M. F. Spear, A. Shriraman, L. Dalessandro, S. Dwarkadas, and M. L. Scott. Nonblocking transactions without indirection using alert-on-update. In Proceedings of the Nineteenth Annual ACM Symposium on Parallel Algorithms and Architectures, SPAA '07, pages 210-220, New York, NY, USA, 2007. ACM.

[117] F. Tabba, M. Moir, J. R. Goodman, A. W. Hay, and C. Wang. Nztm: Nonblocking zero-indirection transactional memory. In Proceedings of the Twenty-first Annual Symposium on Parallelism in Algorithms and Architectures, SPAA '09, pages 204-213, New York, NY, USA, 2009. ACM.

[118] G. Taubenfeld. The black-white bakery algorithm and related bounded-space, adaptive, localspinning and fifo algorithms. In DISC '04: Proceedings of the 23rd International Symposum on Distributed Computing, 2004.

[119] P. K. Vincent Gramoli and S. Ravi. Sharing a sequential data structure: correctness definition and concurrency analysis. 4th Workshop on the Theory of Transactional Memory, Madeira, Portugal, 2012 .

[120] W. E. Weihl. Commutativity-based concurrency control for abstract data types. IEEE Trans. Comput., 37(12):1488-1505, 1988.

[121] G. Weikum. A theoretical foundation of multi-level concurrency control. In PODS, pages 31-43, 1986.

[122] G. Weikum and G. Vossen. Transactional Information Systems: Theory, Algorithms, and the Practice of Concurrency Control and Recovery. Morgan Kaufmann, 2002.

[123] M. Yannakakis. Serializability by locking. J. ACM, 31(2):227-244, 1984. 



\section{Papers}

The content of the thesis is based on the following tech reports and publications.

\section{Tech reports}

P. Kuznetsov and S. Ravi. Progressive transactional memory in time and space. CoRR, abs/1502.04908, 2015.

P. Kuznetsov and S. Ravi. Why transactional memory should not be obstruction-free. CoRR, abs/1502.02725, 2015.

D. Alistarh, J. Kopinsky, P. Kuznetsov, S. Ravi, and N. Shavit. Inherent limitations of hybrid transactional memory. CoRR, abs/1405.5689, 2014.

V. Gramoli, P. Kuznetsov, and S. Ravi. Optimism for boosting concurrency. CoRR, abs/1203.4751, 2012.

P. Kuznetsov and S. Ravi. On partial wait-freedom in transactional memory. CoRR, abs/1407.6876, 2014.

H. Attiya, S. Hans, P. Kuznetsov, and S. Ravi. Safety of deferred update in transactional memory. CoRR, abs/1301.6297, 2013.

P. Kuznetsov and S. Ravi. On the cost of concurrency in transactional memory. CoRR, abs/1103.1302, 2011.

\section{Publications}

P. Kuznetsov and S. Ravi. Progressive transactional memory in time and space. CoRR, abs/1502.04908, 2015. To appear in 13th International Conference on Parallel Computing Technologies, Russia.

H. Attiya, S. Hans, P. Kuznetsov, and S. Ravi. Safety and deferred update in transactional memory. In R. Guerraoui and P. Romano, editors, Transactional Memory. Foundations, Algorithms, Tools, and Applications, volume 8913 of Lecture Notes in Computer Science, pages 50-71. Springer International Publishing, 2015.

P. Kuznetsov and S. Ravi. On partial wait-freedom in transactional memory. In Proceedings of the 2015 International Conference on Distributed Computing and Networking, ICDCN 2015, Goa, India, January 4-7, 2015, page 10, 2015.

H. Attiya, S. Hans, P. Kuznetsov, and S. Ravi. Safety of deferred update in transactional memory. 2013 IEEE 33rd International Conference on Distributed Computing Systems, 0:601-610, 2013.

V. Gramoli, P. Kuznetsov, and S. Ravi. From sequential to concurrent: correctness and relative efficiency (ba). In Principles of Distributed Computing (PODC), pages 241-242, 2012.

P. Kuznetsov and S. Ravi. On the cost of concurrency in transactional memory. In OPODIS, pages 112-127, 2011. 


\section{Workshop papers}

D. Alistarh, J. Kopinsky, P. Kuznetsov, S. Ravi, and N. Shavit. Inherent limitations of hybrid transactional memory. 6th Workshop on the Theory of Transactional Memory, Paris, France, 2014.

P. K. Vincent Gramoli and S. Ravi. Sharing a sequential data structure: correctness definition and concurrency analysis. 4th Workshop on the Theory of Transactional Memory, Madeira, Portugal, 2012.

P. K. Hagit Attiya, Sandeep Hans and S. Ravi. What is safe in transactional memory. 4th Workshop on the Theory of Transactional Memory, Madeira, Portugal, 2012.

Concurrently, I was also involved in the following paper whose contents are not included in the thesis.

V. Gramoli, P. Kuznetsov, S. Ravi, and D. Shang. A concurrency-optimal list-based set. CoRR, abs/1502.01633, 2015. 